\title{
AJTES
}

\section{ALBANIAN JOURNAL OF TRAUMA AND EMERGENCY SURGERY}

\section{Focus on \\ "Trauma and Emergency Surgery" and not only...}

Official publication of 


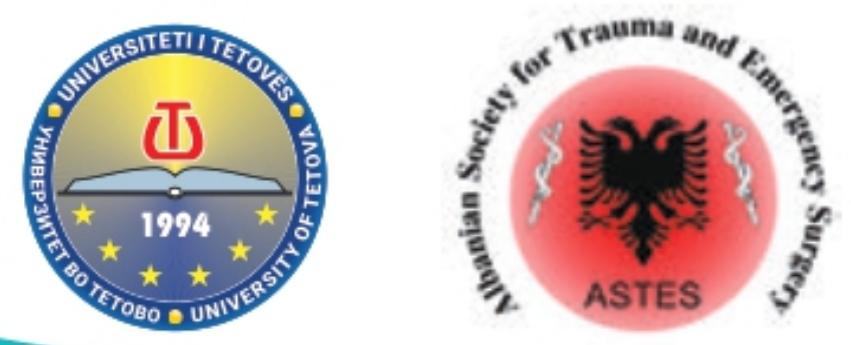

\section{ABSTRACTS BOOK}

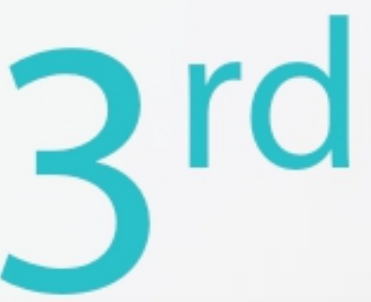

\section{International}

Scientific Conference

of the Faculty of

Medical Sciences
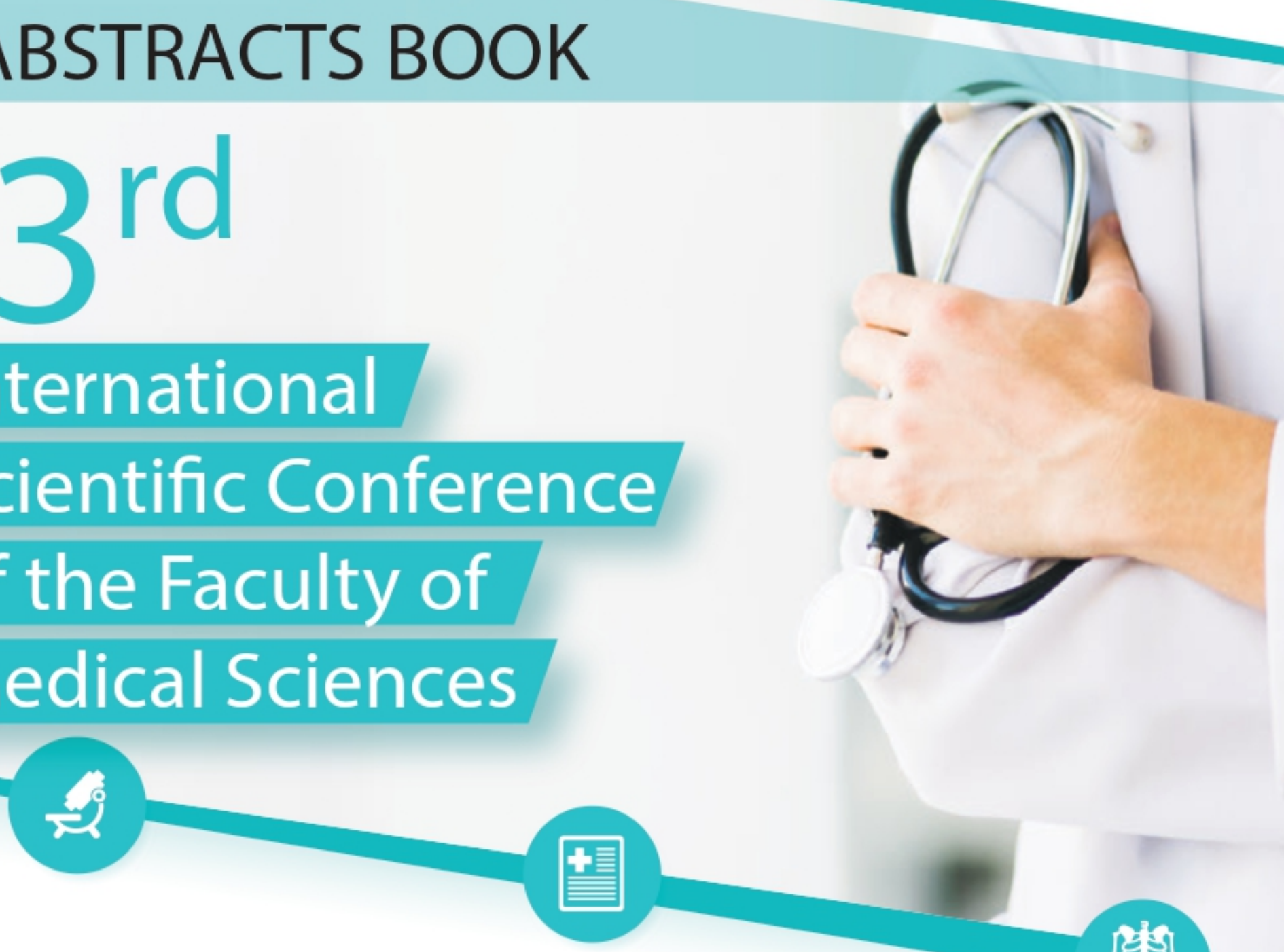

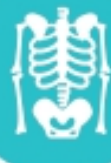

2 nd Annual Albanian Congress of Trauma \& Emergency Surgery

08-10 November, 2018

OHRID, MACEDONIA 


\section{Focus on \\ "Trauma and Emergency Surgery" and not only...}

official publication of

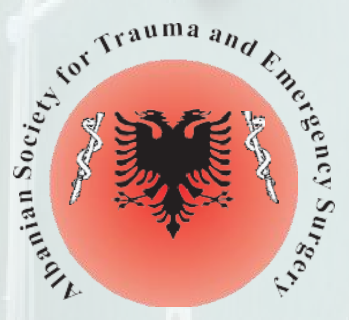

ISSN: 2521-8778 (print version)

ISSN: 2616-4922 (electronic version) 
Albanian Journal of Trauma and Emergency Surgery - AJTES

\author{
Official Publication of the Albanian Society for Trauma and \\ Emergency Surgery - ASTES
}

Chairman of the editorial board: Prof. Rifat LATIFI, MD, FACS, FICS (USA)

AJTES Executive Director (CEO): Prof. Asc. Agron Dogjani M D, PhD, FACS (ALBANIA)

\author{
Editor in Chief: Edvin SELMANI, MD, PhD (ALBANIA) \\ Prof. Asc. Rustem CELAMI MD, Ph.D. \\ Deputy Editor (ALBANIA) \\ Prof. Lutfi ZYLBEARI MD, Ph.D. \\ Enver FEKAJ, M.D, Ph.D. \\ Deputy Editor (Macedonia) \\ Deputy Editor (Kosovo)
}

\title{
National Editorial Board
}

Prof. Arben GJATA, MD, PhD, University of Medicine, Tirana, Albania Prof. Xheladin DRACINI, MD, PhD, University of Medicine, Tirana, Albania Prof. Nikollaq KACANI, MD, PhD, University of Medicine, Tirana, Albania Prof. Etmont ÇELIKU, MD, PhD, University of Medicine, Tirana, Albania Prof. Arben BEQIRI, MD, PhD, University of Medicine, Tirana, Albania Prof. Ilia MAZNIKU, MD, PhD, University “Alexander Xhuvani”, Elbasan, Albania Prof. Arvin DIBRA, MD, PhD,

University of Medicine, Tirana, Albania Prof. Skënder TOPI, MD, PhD, University "Alexander Xhuvani", Elbasan Albania Prof.Asc. Agron DOGJANI,MD, PhD, FACS, University Hospital of Trauma, Tirana, Albania Prof.Asc. Fadil GRADICA, MD, PhD, University of Medicine, Tirana, Albania Prof. Gjergji THEODHOSI, MD, PhD, University of Medicine, Tirana, Albania Prof. Jovan BASHO, MD, PhD, University of Medicine, Tirana, Albania Prof. Krenar PREZA, MD, PhD, University of Medicine, Tirana, Albania Prof. Pirro PRIFTI, MD, PhD, University "Alexander Moisiu”, Durres, Albania Prof. Maksim ÇELA, MD, PhD, University of Medicine, Tirana, Albania Prof.Asc. Sokol BUBA, MD, PhD, University of Medicine, Tirana, Albania
Prof.Asc. Edmond ZAIMI, MD, PhD, University of Medicine, Tirana, Albania

Prof.Asc. Ridvan ALIMEHMETI, MD, PhD, University of Medicine, Tirana, Albania

Prof.Asc. Rudin DOMI, MD, PhD, University of Medicine, Tirana, Albania Prof.Asc. Bajram BEGAJ, MD, PhD, University Hospital of Trauma, Tirana, Albania

Prof. Tritan SHEHU, MD, PhD, University of Medicine, Tirana, Albania Prof. Asc. Besim Boci, MD, PhD, University of Medicine, Tirana, Albania Prof.Asc. Skënder BRATAJ, MD, National Center of Emergency Medicine, Albania Prof.Asc. Artid DUNI, MD, PhD, University of Medicine, Tirana, Albania Artid LAME, MD, PhD, University of Medicine, Tirana, Albania Leart BERDICA, MD, PhD, University of Medicine, Tirana, Albania Arben RROJI, MD, PhD, University of Medicine, Tirana, Albania Myzafer KACl, MD, PhD, University of Medicine, Tirana, Albania

Shkelzen OSMANAJ, MD, PhD, University Hospital of Trauma, Tirana, Albania Zamir DEMIRAJ, MD, PhD, University Hospital of Trauma, Tirana, Albania Gjergj SEMINI, MD, PhD, University of Medicine, Tirana, Albania 


\section{International Editorial Board}

Prof. Rifat LATIFI, MD, FACS, FICS (USA) Prof. Selman URANUES, MD, FACS (Austria) Ass.Prof. Pantelis VASSILIU,MD, PhD, FACS, (Greece) Ruben PERALTA, MD, FACS, (USA) Prof.Mauro ZAGO, MD, FEBS, FACS, (Italy) Prof. Bellal A. JOSEPH, MD, FACS, (USA) Prof. D A Archivio LAFRANCO, MD, DDS, (Italy) Prof. Orhan ALIMOĞLU, MD, (Turkey) Prof. Mehmet Kurtoğlu, MD, PhD, (Turkey)
Ass. Prof. Bogdan DIAKONESKU, MD, PhD, (Romania) Prof. Ayman El-MENYAR, MD, MRCP, FRCP, FESC, FACC, (QATAR) Kastriot HAXHIREXHA, MD, PhD, (Macedonia) Prof. Mehmet ERYILMAZ,MD, MBAH, (Turkey) Danny Daphnis, MD, PhD, (Greece) Basri LENJANI, MD, PhD, (Kosovo) Skender ZATRIQI, MD, PhD, (Kosovo) Faton HOXHA, MD, PhD, (Kosovo)

\section{Advisory Expert}

Prof. Eliziana PETRELA, MD, PhD, University of Medicine, Tirana, Albania.

\section{Assistant Editors}

Admir MUSTAFA, MD

Ferizat Dika - HAXHIREXHA, MD, PhD, (Macedonia)

Erion SPAHO, MD

Sonja SARACI, MD
Hysni BENDO

Amarildo BLLOSHMI

Mail Address: Str. Lord Bajron, 1026, Tirana, Albania

Electronic Address: E-mail.: secretary@astes.org.al ajtesjournal@gmail.com 
Albanian Journal of Trauma and Emergency Surgery is the official publication of Albanian Society for Trauma and Emergency Surgery - ASTES

\section{Structure and Format}

AJTES is a periodical journal includes editorials, reviews, original articles, case reports, short report, ideasand opinions, bookreviews, perspectives, seminars, symposium and mini- symposium, ethics and rights, health care policy and management, practice guides. The structure of each edition of the publication comprises section categories determined by Editor and reflects the views of the Editorial Board.

\section{Editorial Expertise}

Guidance from Editorial Board consists of leading authorities from a variety and respective fields of medical science. Peer review-accomplish by expert selected for their experience and knowledge of a specific topic.

\section{Important notice for Author}

All manuscript must be submitted to the Editorial Office through electronic post; e-mail: contact@ astes.org.al. Please check the last page of each edition "Instruction for Author": The work shall not be published elsewhere in any language without the written consent of AJTES. The articles published in this journal are protected by copyright, which cover translation rights and the exclusive right to repro- duce and distribute all of the articles printed in the journal. No material published in the journal may be stored on microfilm or videocassettes or in electronic databases and the like or reproduced photographically without the prior written permission of AJTES.

\section{Permissions}

For information on how to request permissions to reproduce articles/information's from this journal, please contact Editorial Office.

\section{Advertising policies}

The journal accepts displayed and classified advertising. Frequency discounts and special position are available. The journal reserves the right to reject any advertisement considered unsuitable according to the set policies of the journal. The appearance of advertising or product information in the various sections in the journal does not constitute an endorsement or approval by the journal and/or its publisher of the quality or value of the said product or of claims made for it by its manufacturer.

\section{Disclaimer}

The information and opinions presented in the Journal reflects the views of the author and not of the Journal or its Editorial Board or the Publisher.

Publication does not constitute endorsement by the journal. 
Albanian Journal of Trauma and Emergency Surgery - AJTES

AJTES Volume 2, Number 2, Supplement 2, 2018.

ISSN: 2521-8778 (print version)

ISSN: 2616-4922 (electronic version)

https://doi.org/10.32391/aites.v2i2.2.155

\section{Abstracts BOOK}

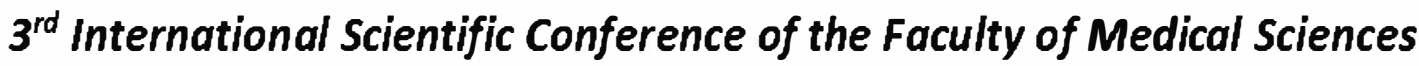

$\&$

$2^{\text {nd }}$ Annual Albanian Congress of Trauma and Emergency Surgery - ACTES 2018

\section{8 -10 November 2018 \\ Ohrid, Republic of North Macedonia}

President of Honour:

RIFAT LATIFI

Congress President:

VULLNET AMETI, AGRON DOGJANI, ARBEN GJATA, KASTRIOT HAXHIREXHA, LUTFI ZYLBEARI

Organized by:

Faculty of Medical Sciences, University of Tetovo, Republic of North Macedonia

Albanian Society for Trauma \& Emergency Surgery - ASTES 


\title{
Official Publication of the Albanian Society of Trauma and Emergency Surgery - ASTES
}

\author{
Aims and Scope \\ Our aim is to promote \\ interest, knowledge and \\ quality of care in \\ emergency and trauma \\ surgery. ASTES was \\ formed in 2017, it seeks to \\ promote best practice in the \\ provision of emergency and \\ trauma surgery and acute \\ care surgery, from pre- \\ hospital care through \\ diagnosis, intervention and \\ intensive care to \\ rehabilitation. This is \\ supported by Countrywide \\ \& International \\ collaboration, scientific \\ research, development and \\ delivery of training courses, \\ and the work of the \\ specialist sections \\ (Polytrauma, Visceral \& \\ Chest Trauma, Skeletal \\ Trauma \& Sports Medicine, \\ Neurosurgical, Anesthesia - \\ Reanimation, Acute Care \\ Surgery, ENT \& \\ Ophthalmology \& \\ Maxillofacial, Radiology, \\ Nurse service, Disaster \& \\ Military Surgery...etc.) \\ ASTES holds an annual \\ scientific meeting - the \\ Albanian Conference for \\ Trauma and Emergency \\ Surgery (ACTES) and \\ produces a bi-annual \\ journal - the Albanian \\ Journal for Trauma and \\ Emergency Surgery
}

ORAL PRESENTATIONS Contents: 


\section{ORAL \\ PRESENTATIONS}

\section{The Injury Pattern in Polytrauma. Distribution of Injuries Based on the Analysis of more than 500 Patients.}

Vilmos VÉCSEI ${ }^{1}$, Stephan FRENZEL ${ }^{1}$

${ }^{1}$ Dept. for Orthopedics and Trauma Surgery, Medical University Vienna, Austria.

\begin{abstract}
Introduction: although the term "polytrauma" has been in use for decades, no generally accepted definition exists.

Our definition has been until 2010: a combination of injuries where one, or the combination where one, or the combination of injuries himself is directly life threatening $g_{2}$ in detail is meant: injury to two body cavities, or injury to one cavity committed by two long bone fractures, where spine and unstable pelvis has counted similarly as an injury to a cavity organ.

Since 2013 a new definition has been established, the so called Berlin definition.

„A polytrauma means significant injuries of three or more points (AIS) in two or more different anatomic AIS regions in conjunction with one or more additional variables from 5 physiologic parameters:

- Hypotension (systolic blood pressure $<=90 \mathrm{~mm} \mathrm{Hg}$ ),

- Level of consciousness (Glasgow Coma Scale (GCS) $<=8$,

- Acidosis base excess $<=-6.0$ ),

- Coagulopathy (international normalized ratio $>=1.4 /$ partial thromboplastin time $>=40$ seconds) and age $>=70$ years).
\end{abstract}

This definition fits the reality perfectly.

The Dept. for Trauma Surgery of the Medical University of Vienna, Austria, presents itself. A level I trauma center, located in the General Hospital, as a special unit for the treatment of heavily injured in the eastern region of Austria.

In a 10-year period 501 polytraumatized patients have been admitted.

We looked to the injury pattern in two 5year periods (A, B) and compare the numbers with a group of polytraumatized 10 years later(C).

The injury pattern, the injury severity follows the cause of the injury and determines the outcome. AIS-comparison survivors vs. dead

The pattern of Injury follows local conditions, has an important function for accident prevention and is not a factor for a comparative study.

Key Words: Polytrauma, Definition of Poltytrauma,

\section{Current Management of Complex Vascular Abdominal Trauma: Priorities and Techniques}

Rifat LATIFI ${ }^{1}$

${ }^{I}$ New York Medical College, School of Medicine Director, Department of Surgery, Westchester Health Network, Department of Surgery, New York Medical College. Valhalla, NY, USA.

\section{Abstract}

Vascular trauma is the most common cause of mortality and significant morbidity of patient sustaining penetrating or blunt abdominal trauma. To this end, the ability to deal with injuries to the vascular tree of the abdomen in the most expeditious manner is a must for every trauma surgeon, as well as for any general surgeon who deal with trauma patients. Knowledge of anatomy and surgical techniques that will provide rapid exposure to aorta and aortic branches, vena cava, portal vein and their branches, proximal and distal control without causing more injury of any vessel, combined with skillful ability to provide definitive or temporary revascularization and establish blood flow to the intestines, liver and other solid organs, or lower extremities is essential. There are number of well-established 
exposures and techniques that have stood the test of time and will help treating trauma patients with massive vascular injuries and should be part of every surgeon's armamentarium and trauma center. In most recent years, however, new techniques such as temporary shunting, stenting and embolization of solid organs (liver, spleen and pelvic embolization primarily) have become common and have been proven lifesaving adjuncts to trauma surgery. While multidisciplinary approach is often required, the initial approach by the trauma/general surgeon must be well focused, planned and systematic, and should avoid adventuristic moves or decisions. While damage control surgery may be part of this armamentarium, the named vessels should be addressed surgically. I will describe few of these techniques during this presentation.

\section{Surgical Priorities in The Treatment of Polytraumatized Patients with Orthopaedic Trauma}

Jordan SAVESKI ${ }^{1}$, Neda TRAJKOVSKA ${ }^{1}$, Ilir HASANI $^{1}$.

${ }^{1}$ Clinical Hospital Acibadem Sistina, Skopje, MACEDONIA. University Clinic of Traumatology, Medical Faculty, Skopje, MACEDONIA

\begin{abstract}
Introduction: Determination of surgical priorities, extend of surgery and planning and optimal timing for fracture stabilization is essential in the treatment of polytrauma patients with multiple fracture. For most patients (hemodynamic stable) the concept for early total care (ETC) has an advantage. An unstable and extremis patient should be resuscitated and adequately stabilized before receiving definitive treatment. Damage control orthopaedic (DCO) surgery increases the chances for survival in patient at risk, with severe multiple fracture with chest and head injury. The decision whether to perform initial temporary of definitive fixation in the borderline patient is based on clinical condition.
\end{abstract}

Purpose: The purpose of this study is to present our principles based on the treatment experience based on 820 polytraumatized patient with orthopaedic trauma

Material and method: A total of 820 polytraumatized patients were treated between 2000 and 2014 year. The severity of injury (ISS) was 28 range from 16 to 75 years and GCS 9 (range from 3 - 15). The treatment management was done between 4 different periods: acute or resuscitation (1-3 hours), primary or stabilization (1-72 hours), secondary or regeneration (3-8 days) and tertiary or rehabilitation (after the $8^{\text {th }}$ day). The primary goals in the resuscitation phase was to establish adequate ventilation, to maintain the circulation according ATLS - ABCs protocol (golden hour) and to access the global neurological status.

Results: Retrospectively were compared 2 groups of polytraumatized patient with femoral shaft fractures according the treatment strategies for femoral shaft fractures. Gorup A - intramedullary nailing (IMN) as a ETC and group B - DCO surgery. In group B ISS was higher (32) compared to group A (22). The patient in group B required more fluids (14,21 vs $8,21)$ and blood $(2,2$ vs 1,3$)$ compared to group A in the initial 24 hours. Thoracic, abdominal, head injuries were present in significantly higher number in group $\mathrm{B}$ $(24,2 \%)$ than in group A $(12,4 \%)$. There was significantly higher incidence of ARDS in group A $(18,2 \%)$ than in group B $(8,6 \%)$. Also MOF was significantly lower in group B $(7,4 \%)$ than in group A $(12,1 \%)$. There were 3 unexpected deaths and 2 conscious worsening in patients with head injury in group A.

\section{Conclusion:}

Determination of surgical priorities and time of surgery is essential in the treatment of polytrauma patients. Early fracture fixation is respected principle in stable polytrauma patients with orthopaedic trauma. DCO surgery is strongly indicated in unstable and extreme polytrauma patients with multiple fractures. 


\section{Controversies on definition, assessment and workflow, with overview of damage control modern concept of treatment of 'real' polytrauma patient.}

Ilir HASANI ${ }^{1}$

${ }^{1}$ General Surgeon-Traumatologist, Traumatology Orthopaedic Surgery, Anesthesy, Intensive Care and Emergency Centre, Skopje, MACEDONIA.

\section{Abstract}

Historically, politrauma is associated to complex and contraverse issues beginning from definition, assessment, primary and secondary approach, and also big controversies on time, type and amount of treatment, as referred in the literature as second hit.

We have reviewed the literature on definition of politrauma and find a lot of controversies and disagreement between surgeons. We present the final definition that is still very hardly implementable in our routine work.

A lot of work has been done on standardizing the assessment and approach of the politraumatized patient on the last century that has been summarized on very precise ATLS protocol. Since the Computerized tomography has became more available for the politraumatized patients in the Emergency Room worldwide, the discussion of favoring the whole body scan on the Emergency Room vs. ATLS protocol has attract the attention of medical society. Arguments of both statements will be presented.

Recently, withdraw from aggressive behavior of surgeon and anesthesiologist, preventing adverse outcome due to inability of injured body to bear the second iatrogenic attack, has become a golden standard on treating seriously multiply injured patient, so called Damage control concept on politraumatized patient.

\section{Organization of Trauma Care Services in Traumatized Patients}

Arben GJATA ${ }^{1}$

${ }^{1}$ Rector University of Medicine Tirana, Albania.

\begin{abstract}
Traumas represent one of the most important clinical and diagnostic pathologies that often make extreme emergencies.
\end{abstract}

In developed countries, under conditions of peace, trauma constitutes the first cause of death for under 40s and is a cause for a high number of permanent disability.

Trauma means a transfer of energy, which is due to the interaction of physical or chemical agents with the organism. In this view, when talking about trauma, we are talking about different events, such as road accidents, work or homes, but also burns or lesions, from cold-fire aggression to those with firearms.

The role of the surgeon in the trauma team is essential. It should provide multidisciplinary care to reduce diagnostic time and optimize therapeutic procedures.

In some countries, assistance at the venue is provided by medical teams, while in the US from paramedics, who are trained personnel for this purpose.

The out-hospital phase is as important as the hospital. The dynamics of the accident, the injuries sustained by the accident, the vital parameters and the therapeutic procedures of primary assessment, basic life support (BLS) or advanced trauma life support (ATLS) should be evaluated.

During transport, the patient's condition should be communicated to the team.

The time of transport is important for the trauma team because it gives time to prepare for the accidental reception.

The team besides the general surgeon, the resuscitator and the imaging may also need other specialists who are called depending on the situation.

Transferring the patient from the hospital to the hospital is an important time to get all the information that is needed and available. 
Once the hospital team becomes responsible for the traumatized person, the primary and secondary examination of the patient is re-examined, to be passed later in the diagnostic phase.

The patient is continuously monitored with the ECG, arterial pressure, oxygen saturation. Ventilation parameters should be measured in the case of intubated patients.

Any deviation from these well-defined protocols, according to numerous studies, is associated with significant increases in morbidity and mortality.

\section{Trauma Surgeon vs General Surgeon in Trauma Management}

\section{Mauro ZAGO ${ }^{1}$}

${ }^{I}$ General Surgery Dept, Minimally Invasive Surgery Unit, Head Policlinico San Pietro - BOLOGNA ITALY*

\begin{abstract}
As Medicine adapts to the 21st century, new specialties arise. In the management of trauma, two models have been opposed in the past: on one side, a Trauma Surgeon based system, with specialists fully devoted to trauma care, often able to fix skeletal trauma too; on the other hand, blended systems with General Surgeons dealing with both elective and emergency surgery and trauma patients.

The evolution of technology, of the epidemiology of trauma, and of the trauma systems and networks entailed the emerging of the concept of Acute Care Surgery. In the vast majority of Countries, this new specialist semms to better fit with the needs of both patients and health organization.

Who is the Acute Care Surgeon? What is his minimal educational and technical background? How can interact with the other medical specialists playing around a trauma patient?
\end{abstract}

\section{Management of Trauma Patient in the Hospital Settings.}

Agron DOGJANI ${ }^{1}$, Hysni BENDO, Amarildo BLLOSHMI

${ }^{1}$ University Hospital of Trauma, Tirana, Albania

${ }^{2}$ University of Medicine, Tirana, Albania

\begin{abstract}
Introduction: Trauma can be defined as a 'blunt or penetrating external force exerted on the body resulting in injury'. Traumatic injury is a leading cause of death globally among persons under the age of 45 years. Over 5 million deaths occur each year as a result of injuries, representing $9 \%$ of the world's mortality. Non-fatal injuries are among the leading global cause of emergency department (ED) visits, hospitalizations and long-term morbidity, accounting for a large part of health systems workload. Although road injuries, falls and self-harm are the top three causes of injury burden globally, their relative importance may differ according to the region.

The initial assessment and management of traumatized patients should take place in a specialized area of an emergency department or a specialized trauma center. The time of injury is the essence of survival for life-threatening trauma.
\end{abstract}

Appropriate initial care at the scene affects the morbidity and mortality of the traumatized patient. Prolonged transport time or inadequate hospital care increases the demand for early restoration of rapid tissue perfusion before the physiological changes of the injured patient reach the hospital. This presentation addresses the management of traumatized patients after these patients reach an emergency department at a regional / municipal hospital or a traumatic center.

Keywords: primary trauma center, traumatized patients, injured patient, life-threatening trauma 


\section{History and Current \\ Epidemiological Profile at UHT, Tirana, Albania.}

Luan NIKOLLARI ${ }^{1}$, A. BEJLERI ${ }^{1}$

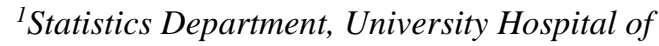

Trauma, Tirana, Albania.

\begin{abstract}
Background: Despite advances in technology, hospital infrastructure, and human resources, trauma continues to be a major public health problem not only in Albania. The purpose of the study is to give a complete overview of the history of this institution from the most important in Albania as well as comparative data of traumatic epidemiology.
\end{abstract}

Materials and Methods: This is a retrospective study conducted in the period January 2013 - 2017. In this study, data are analyzed for hospital activity, frequency by region, age group, incidence, affected body regions, and mortality.

Results: A total of 173,411 patients with trauma were presented during this time frame, about 34,602 patients per year, 95 patients per day. Out of them about 3200 patients $(8.7 \%)$ in year needed hospital treatment. $21 \%$ of the patients were treated in intensive care unit and the rest in other services. Most of the patients were males $(68 \%)$ with the mean age $15 \pm 65$ years. $72 \%$ of injuries were attributed blunt trauma. The most affected body regions resulted: the cranial-spinal trauma with $50.3 \%$ injuries, followed by the trauma of extremities and pelvis $29.4 \%$, and by polytrauma $10.6 \%$. Mortality in ICU were $18 \%$ and in clinics $5.1 \%$. Motor vehicle collisions were the first cause of death (44\%) in 2016 and up to $48 \%$ for 2017.

Conclusion: Trauma in Albania has propensity to rise. Awareness-raising programs for pedestrian and driver education, road safety improvement are necessary. More attention and promote among healthcare professional to continuing medical education from the government is needed.

Keywords: epidemiology, trauma, UHT, Albania

\section{Quality Control in Heath Care and in Trauma Surgery it should be Our Mission.}

Skender ZATRIQI ${ }^{1}$, Ferat SALLAHU ${ }^{1}$, Enver FEKAJ $^{1}$, Violeta ZATRIQI ${ }^{1}$

${ }^{I}$ Department of Abdominal Surgery, University Clinical Centre of Kosovo, Pristina, KOSOVO

\begin{abstract}
Introduction: All patients are entitled to good standards of practice and care from their doctors. Essential elements of this are professional competence, good relationships with patients and colleagues, and observance of professional ethical obligations Health care organizations are accountable for continuously improving the quality of their services and safeguarding high standards of care by creating an environment in which excellence in clinical care will flourish.
\end{abstract}

Aim of the work; we assessed our institutions for terms quality assurance control how much is dispersed this concept and is there available documentation

Good clinical care must include an adequate assessment of the patient's condition, based on the history and clinical signs and, if necessary, an appropriate examination.

Quality standards must be defined by each department and provide measurable endpoints. Departments should ensure that they are aware of their Trust's risk management policy and adhere to it on all occasions

Results; There is capacity and resources in human and god prepared documentation but institutional memory was lost and previous ministers that were developing this institution new ones do not have any information about scope and job done previously. Quality control is for us a new concept and difficult to implement. A system of accreditation for specific interventions should be introduced guaranteeing their state-of-the-art application. There is urgent need to implement the QCP in order to meet standards in need for providing good services in order to fulfill requirements for 
Licensing accreditation and finally for gaining and profiting

Keyword: Quality Control, Kosovo perspective, quality assurance, Trust's risk

\section{The Situation of Violence in High School of Shkodra Region.}

Zamira SHABANI ${ }^{1}$, Irena SHALA ${ }^{1}$, Vera GJINAJ $^{1}$, Emiljano PJETRI ${ }^{1}$, Kilda GUSHA ${ }^{1}$ ${ }^{1}$ University "Luigj Gurakuqi" Faculty of Natural Sciences, Department of Nursing, Shkoder, Albania

\begin{abstract}
Violence is considered any behavior or attitude that harms the physical, emotional, and sexual well-being of one or more persons and affects the termination of the individual's normal development. Violence at school includes behavior such as: victimization of a child and teachers, rape of a child and / or a teacher, physical and psychological harassment, victimization cybernetism, cyber threat and cybernetics, controversy, bullism, classes of physical and psychological harm teachers and students, behavior and activities, which are derivatives of different cultures, sexual violence and other border violations, and the use of weapons in school environments. The main forms of violence in school are: teachers towards students, students to teachers, students to students.

This is a punctual, cross-sectional study. In this study was used the Standardized Questionnaire Based on the Global SchoolBased Student Health Study (GSHS) 2003, Extended Based Questions on Violence and Injury Module Violence. The survey was conducted to high school students in Shkodra Region. Research data are analyzed through statistical method with microsoft office 2007. The questionnaire was administered during January-February 2018.
\end{abstract}

Key words: violence, teachers, students, school, Shkoder.

\section{Managing the Patient Evidence Base through Trauma Cases in University Hospital of Trauma, Tirana, Albania.}

Rudina DEGJONI ${ }^{1}$

${ }^{1}$ Head of Patient Department, Health Care

Insurance Fund of Albania Tirana, ALBANIA

\section{Abstract}

The level of management of traumatic health care services in Albania as a service of the University Hospital of Trauma, in Emergency Care differs in relation to other university hospitals across the country, mainly due to changes in the public health of the population.

The article aims to present the management of traumatic patients at the University Hospital of Trauma, Tirana, Albania, providing examples of the number of cases that have been addressed in the spread of this disease as epidemiology in Albania. The literature review and data statistics will be carried out by using the hospital records that will be used for further research in Albania.

In addition, there is a study of international reports on Trauma management issues, trauma care and quality in Trauma. Much of the results consider the necessity of adopting a comprehensive traumatic system, focusing on quality management and improving trauma care to increase survival and reduce complications to the wounded. Urban conditions and public health policies are the most important factors in developing and implementing programs to improve emergency trauma care services. The heterogeneity of the population and its geographical distribution, the complexity of road accidents, are challenging factors for quantitative research on trauma management and the effectiveness of its care. Staff training in all areas, combined with the existence of modern vehicles and equipment, means of communication and timely transfer of the ambulance, contribute to the treatment of patients with urgent trauma, life saving and prevention of all types of disabilities. 
Keywords: trauma, UHT, Albania, epidemiology, patient, evidence

\section{Devil Stands in Details, when Colon is not long enough in Laparoscopic LAR? Tips and Tricks of a Safe Anastomosis.}

\author{
Arben BEQIRI ${ }^{1}$, Antonio CATONA ${ }^{2}$ \\ ${ }^{1}$ General Surgery Service, University Hospital \\ Center "Mother Teresa" Tirana, Albania. \\ Surgery Service American Hospital, Tirana, \\ ALBANIA. \\ ${ }^{2}$ Surgery Service American Hospital, Tirana, \\ ALBANIA.
}

\begin{abstract}
Every time you are doing a left colectomy for a descending colon cancer after taking down IMA and IMV and mobilizing splenic flexure you run into some risk of tension on the low anastomosis. The transverse colon will not reach the rectum without tension on the middle colics.

In this lecture we discus all options available to get through this difficult situation in colorectal surgery.

Our experience with laparoscopic colorectal surgery at American Hospital is demonstrated as well as part of the progress made in this institution in the last year.
\end{abstract}

\section{Laparoscopic Surgery for Colorectal Cancer}

Nuhi ARSLANI ${ }^{1}$, Stojan POTRČI ${ }^{1}$

${ }^{1}$ University Clinical Center Maribor, Department for Abdominal Surgery, University of Maribor, Faculty of Medicine, SLOVENIA

\begin{abstract}
Background; Laparoscopic surgery for colorectal cancer hasundergone a major paradigm shift in the past two decades: from a new technique thought inappropriate for oncologic surgery to one with sufficient scientific scrutiny to establish itself as a good alternative, if not the preferred approach, in suitable cases.

Material and Methods: From 2004 to 2014, we operated on 578 patients who had been
\end{abstract}

admitted for colorectal cancer. We analyzed a variety of data to provide a summary of their survival rates, local and systemic complications. For analysis we used data gathered from preoperative diagnostic tests, reports gathered during operation, reports from pathohistologic review, and reports on complications after surgery and follow up Results; In the case of 573 (out of 578) patients $(99.1 \%)$ we performed resection. R0 was achieved in 551 patients $(96,1 \%)$. $\mathrm{R} 1$ was achieved in 8 patients $(1,4 \%)$.

Local complications were reported in 78 $(13.5 \%)$ patients and systemic complications were reported in $68(11.7 \%)$ Conclusions; Laparoscopic colorectal surgery for cancer has become the gold standard. Laparoscopic colorectal resection for cancer, in experienced hands, can be performed safely and reliably with many short-term benefits to the patients while resulting in at least equivalent long-term outcomes as open surgery, but less conclusively demonstrated benefits include better preservation of cell-mediated immune function and reduced tumor cell proliferation. Laparoscopic resection for rectal cancer can yield the best results in selected cases by specialists in high volume centres.

With advances in surgical techniques, with multimodal-multidisciplinary approach and with the use of total mesorectal excision we experienced a significant improvement in 5year survival rate of patients with colorectal cancer. However there still remains the question for truly optimal care for each patient.

Keyword: colorectal surgery, colorectal cancer, Laparoscopic surgery... 


\section{Open Abscesso - Fistulotomy in Anal Abscesses, Results of Treatment.}

Enton BOLLANO ${ }^{1}$, Dariel THERESKA ${ }^{1}$, Krenar LILAJ $^{2}$

${ }^{1}$ Service of General Surgery, University Hospital Center "Mother Teresa" of Tirana, Albania

${ }^{2}$ Service of Anesthesiology \& Intensive Care,

\begin{abstract}
Introduction: Perianal abscesses usually develop from the proctodeal glands which originate from the inter-sphincteric plane and perforate the internal sphincter with their duct. The abscesses may break through into the anal canal and resolve completely, but they can also spread by a submucosal, inter-sphincteric or trans-sphincteric route and develop into fistulae.
\end{abstract}

Material and Methods: The study was extended over a period of three years. There are 131 patients presenting in the National Proctology Cabinet at Service of General Surgery, University Hospital Center "Mother Teresa" of Tirana, Albania.

The average age of patients was 46.5 years (26-67 years). Most patients were males (89).

For reasons of study all cases were intervened with spinal anesthesia using $2 \%$ lidocaine solution. The intervention was chosen as a gynecologic position. The intervention was performed by the same surgeon.

Diagnosis has been clinically established and for all cases trans-rectal and perianal US is performed to evaluate the extent of abscesses. The average length of complaints resulted 2.8 days ( 2 days 4 days)

In 93 patients (Group A) it was possible to clearly identify the crypto-glandular abnormal origin and open abscesso fistulotomy was performed. In 38 Patients (Group B) drainage of the abscesses with ellipsoid incision and passive drainage was performed.

Postoperative resection resulted 27.8 hours for Group A patients. All Group B patients left after 24 hours from hospital. The reasons for prolonged stay of Group A patients resulted: Pain (14), rectal hemorrhage (4), temperature (2) and urinary retention (3).

Postoperative follow-up was performed by consulting the patients every 15 days up to two months after the intervention.

Results: From group A two patients were exposed with anal fistula clinic, a patient with abscess clinic, two patients with gastric incontinence clinic, six delayed wound epithelial patients. From Group B all patients were presented with an anal fistula clinic.

Conclusion: The technique of open abscess-fistulotomy is quite preferred, as per our results, which also pervade the contemporary trend of their treatment.

Keyword: Anal Abscesses, fistulotomy, criptoglandular, transrectal , perianal abscesses

\section{Appendicitis and Meningocele. A Case Report.}

Arvit LLAZANI ${ }^{1}$, Deniona NUNCI ${ }^{2}$

${ }^{1}$ Surgery Departament, University Hospital Center

"Mother Teresa", Tirana, Albania

${ }^{2}$ Imagery Departament, University Hospital Center

"Mother Teresa”, Tirana, Albania

\section{Abstract}

The anterior sacral meningocele is an anterior sacral defect or a herniation of the meningeal sac from the sacral foramen to the anterior. The anterior sacral meningocele does not regress. Although it may be asymptomatic, some authors have reported infectious complications such as meningitis. Therefore, surgical treatment is recommended. We present a case with classic clinical appendicitis who helped in diagnosing the patient with meningocele. We performed standard appendectomy procedure, the patient was discharged uneventfully. The further evaluation by the neurosurgeon concluded anterior meningocele. Having appendisitis helped him to be diagnosed and further followed for the meningocele pathology. We presented the case for the rarity of the associated pathology. Inflammation of the 
appendix made possible for the patient to be evaluated and diagnosed for the meningocele.

Keywords: appendicitis, meningocele, surgery.

\section{Aorto - Jejunal Fistula associated with an Abdominal Aortic Aneurysm. A Case Report}

Astrit XHEMALI ${ }^{1}$, Adrian NELAJ ${ }^{1}$, Eljona $\mathrm{XHELILI}^{1}$

${ }^{1}$ Department of General Surgery, Mother Theresa Hospital Tirana

\section{Abstract}

Primary aorto - enteric fistula is a rare but serious complication of abdominal aortic aneurysm, and the outcome of surgical treatment is poor. With an incidence less than $1 \%$, according to autopsy studies in general population. More than $80 \%$ of them involve the duodenum, mainly in the third and fourth portions. The jejunum and ileum are only affected in $4 \%$ of cases. Mortality ranges from 33 to $85 \%$, being an early diagnosis the most important prognostic factor. The surgical results depend on pre operative condition of the patient and degree of contamination of the operative field. We present the case report of an aorto - jejunal fistula associated with an abdominal aortic aneurysm, without GI bleeding.

Keywords: Aorto-jejunal fistula, abdominal aneurysm, surgery

\section{Colorectal Cancer in Pregnancy: A Case Report}

Drini SHEHI ${ }^{1}$, Myzafer KACI ${ }^{1}$, Henri KOLANI ${ }^{1}$, Arvin DIBRA ${ }^{1}$, Etmont CELIKU ${ }^{1}$

${ }^{1}$ General Surgeon - University Hospital Center

"Mother Theresa" Tirana, ALBANIA

\section{Abstract}

Introduction: Colorectal cancer in pregnancy is a very rare finding, with very few cases reported in the literature and a reported incidence between $0.002-0.005$ $\%$, since its clinical presentation with signs and symptoms that mimic those of pregnancy, it is unfortunately diagnosed too late, in advanced stages of the disease.

Material and Methods: We report a case of a $16 \mathrm{yr}$ old primigravida female treated in the emergency for an intestinal ileus due to an obstructive rectal cancer.

Conclusion: As there are no guidelines regarding diagnoses and treatment, managing these patients remains a challenge for the clinicians. Herein, we report this case to increase the vigilance of our medical system, aiming an earlier diagnosis and treatment and also a better multidisciplinary approach to it, within Obstetricians, Gastroenterologists, Surgeons and oncologists.

\section{Mesenteric Cysts Associated with Acute Appendicitis. A Case Presentations.}

Floren KAVAJA ${ }^{1}$, Miftari $\mathrm{F}^{1}$, Faton HOXHA $^{1}$, Shemsedin HASHANI ${ }^{1}$, Rrustem MUSA ${ }^{1}$, Arsim AJETI $^{1}$, Ali DEVAJA ${ }^{1}$, Braha B. ${ }^{1}$, Shahini L ${ }^{1}$. ${ }^{1}$ Department of General Surgery, University Clinical Center of Kosovo, Pristina, KOSOVO

\section{Abstract}

Background: Mesenteries and omentum cysts are rare in abdominal surgery, which may also have traumatic background origin, also known as pseudocysts of mesenteries. They are asymptomatic or have a noncharacteristically clinic like: nausea, vomiting, pain, abdominal cramps ore constipation. While the cystic mass is affected in the abdomen in $65 \%$ of the cases, therefore cysts are difficult to diagnose in the early pre-operative course. In the etiology of these cysts important role have, lymphatic disorders which are related to the venous system. In the mesentery of the intestines appear in $60 \%$ of the cases, while the mesenteries of the colon are rarely affected according, to some statistics this happens to $24 \%$ of the laundries, while only $25 \%$ of cases are in retroperitoneal position. The adult population hits one case per 140,000 inhabitants, while at the childhood 
one case per 120,000 inhabitants. The classification of these cysts according to Hitti and Sawicki is done in four groups: cysts of embryonal origin, traumatic, neoplastic and inflammatory origin. They can be unilocular and multilocular. A classification from the immune-histological aspect was made by author De Perrot.

The case of mesenteric cyst in our hospital, a 38-year-old patient diagnosed in front of ultrasonography and pelvic resonance magnetic with contrast, is shown in the works, whereas the cyst is located in the right hemi-abdomen but in it is impossible to find correlation in the surrounding organs. After the preoperative preparation the patient was operated in general anesthesia with open infra umbilical incision. The whole cyst extermination was complete, in addition to appendectomy due to secondary apathy inflammation with more coprolite in its space of operation and postoperative pass smoothly without any complications in the postoperative posture. Removal materials are sent to histopathological examination. Completely removed cysts, it is a condition that the histopathological examination be the exact that determines the origin and treatment of the eventuality of it. Other advantages of removing the total cyst are: recidivist opportunities are smaller, but the likelihood of occurring complications during surgery and postoperative progression is reduced.

Key Words: cysts mesenteric, method of operations and appendectomy

\section{Cranio Facial Fractures and Injuries, Its Management}

Claudio TAGLIA ${ }^{1}$, Alesio BOCARI

'University "La Sapienza” Rome, Italy

${ }^{2}$ Nobel Oro-maxillofacial clinic Tirana Albania

\begin{abstract}
Patients with severe craniofacial injuries need a multidisciplinary approach.

In the cases of politraumatic patients the therapeutic timing, once provided to control emergencies, such as upper airways
\end{abstract}

obstructions and bleeding, must resolve the main problem related to the trauma when possible with an approach involving different specialties.

Aim of the therapy is the anatomical and functional recovery.

When diagnosticaliter is completed and the patient is stabilized the surgical operation should be performed as soon as possible due to the fact the delaying could cause difficulties in reduction of fractures.

Incisions should be performed taking care of the high aesthetical impact of the face.

The evolutions in imaging and in materials for internal fixation allowed in the last decade's better outcomes.

The author's report shows the problems related to the various cranio-facial fractures, describing the most common complications and the surgical treatment for each kind of fracture.

Clinical cases are presented and discussed.

\section{Nasal Bone Traumatic Fracture, Immediate and Long Term Management}

Besim BOCI ${ }^{1}$, Klevis THOMAI ${ }^{1}$

${ }^{1}$ ENT Service, University Hospital Centre, "Mother Theresa" Tirana, Albania

\section{Abstract}

The nasal pyramid is the most trauma exposed facial organ, making it prone to all kind of traumatic fractures such as traffic accidents, sport trauma, and even household injuries.

The diagnosis is confirmed through inspection, palpation, direct rhinoscopy, and radiology.

The immediate management consists of bleeding control, stabilization of the bony pyramid, fixing septal injuries, and closing skin lacerations.

Long term management had to deal with old injuries untreated properly. This is achieved through esthetic and functional rhinoseptoplasty. Best result might require the use of every potential varieties of rhinoseptoplasty, including complete 
bony/cartilaginous reconstruction or rebuilding.

Keywords; nasal trauma, rhinoscopy, rhino-septoplasty, cartilaginous reconstruction

\section{Epistaxis Management in Emergency Settings.}

\section{Klevis THOMAI ${ }^{1}$}

${ }^{1}$ ENT Service, University Hospital Centre, "Mother Theresa" Tirana, Albania

\begin{abstract}
Introduction: The lifetime prevalence of epistaxis is approximately $60 \%$, and 6-10\% of the affected persons need medical care. According to a retrospective study from the United States, 1 to 2 out of 200 visits to the emergency department were due to epistaxis, and about $5 \%$ of the patients had to be admitted for inpatient care. In rare cases, severe bleeding calls for the rapid initiation of effective treatment Epistaxis is a very common situation $(50 \%$ of the world's population) and in most cases it is quite dramatic for both the patient and the health care provider.

Causes of nose bleeding are varied, ranging from trauma, cardiovascular and systemic diseases to the side effects side effects of medications.

Epistaxis management requires a calm and sometimes multidisciplinary approach. In this presentation will address the various possibilities of rapid stopping of haemorrhage to reduce the risk of anemia and create conditions for transport, finding causes, and its final treatment.
\end{abstract}

Conclusion: The treatment of severe or recurrent epistaxis requires the interdisciplinary collaboration of the primary care physician, the emergency physician, the practice-based otolaryngologist, and the hospital otolaryngology service. Uniform guidelines and epidemiological studies on this topic would be desirable.

Keywords; epistaxis, severe bleeding, nose bleeding...

\section{Evaluation and Management of Facial Trauma Patient}

Orjada GASHI ${ }^{1}$, Lorena HAXHIHYSENI ${ }^{1}$, Jakup VRIONI $^{1}$

${ }^{1}$ ENT Service, University Hospital Centre, "Mother Theresa" Tirana, Albania

\section{Abstract}

Background: Maxillofacial injuries are commonly encountered in the practice of emergency medicine. More than $50 \%$ of patients with these injuries have multisystem trauma that requires coordinated management between emergency physicians and surgical specialists in oral and maxillofacial surgery, otolaryngology, plastic surgery, ophthalmology, and trauma surgery.

Material and Methods: There are over 238 cases of trauma-related emergency department (ED) visits in the University Hospital of Trauma in Tirana, each year.

Results: Average patient age was 30 years and $76.8 \%$ of the patients were male. Sixtyfour percent of injuries were attributed to fall or blunt trauma. Open wounds comprised $40 \%$ of injuries. The most common procedure was laceration repair (70\%). The majority of patients $(95 \%)$ were discharged home. Mortality rate was less than $1 \%$. The head, neck and face account for just $12 \%$ of total body surface area, yet injuries to these areas are disproportionally represented. Injuries can range from minor soft tissue lacerations to complex facial fractures, penetrating neck wounds and cranial nerve injury. Similar to the range of injuries, the mechanism of injuries are broad and may include minor falls to armed assault and motor vehicle accidents. In developing countries, motor vehicle accident (MVA) is the most common cause of jaw fracture, whereas, in developed countries, assault is the most common cause and MVA is the second most common cause.

Conclusion: Varying from simple, common nasal fractures to gross combination of the face, management of such injuries can be extremely challenging. 
Therefore, trauma to the maxillofacial region mandates special attention. Important sensory systems are contained within the face (e.g. vision, auditory, somatic sensation, gustatory, olfaction and vestibul ar). Also, vital structures in the head and neck region are intimately associated (airway, blood vessels, nerves and gastrointestinal tracts). Lastly, the psychological impact of disfigurement can be devastating.

Management of maxillofacial trauma is complex and requires a comprehensive and accurate diagnostic and treatment plan. It is also important to consider the biologic, functional, aesthetic and economic aspects, as well as the patient's desire.

Keyword: Maxillofacial injuries, facial fractures, penetrating neck wounds...

\section{Importance of Knowledge and Attitude of Emergency Physicians about the Management of Traumatic Dental Injuries in Emergency Departments.}

Orjada GASHI ${ }^{1}$, Marion $\mathrm{SAKO}^{1}$, Lorena

HAXHIHYSENI $^{1}$, Blerina BREGU ${ }^{1}$

${ }^{I}$ Orofacial Surgery Service, University Hospital of Trauma, Tirana, Albania.

\begin{abstract}
Background: Hospital emergency departments are confronted with managing dental emergencies of both traumatic and non-traumatic origin. However, the literature suggests inadequate knowledge of the management of traumatic dental injuries among medical professionals. The aim of the study was to evaluate the knowledge of ER physicians with different specialties, experience and hospital sectors for the management of avulsed teeth in the emergency rooms of University Hospital of Trauma in Tirana. It also covers their attitude towards receiving further education on tooth avulsion management.
\end{abstract}

Material and Methods: A cross-sectional survey was conducted at the emergency rooms of University Hospital of Trauma on
Tirana. A self-administered questionnaire consisting of 31 multiple choice questions assessing both knowledge and attitude was distributed to the physicians who were working in the ER departments.

Results: Response rate was $81.33 \%$. Data revealed that $58.9 \%$ of the respondents did not have prior knowledge about avulsion. $70 \%$ of participants showed willingness to replant the tooth; however, $60 \%$ would not do it by themselves. $40 \%$ of the physicians did not know the importance of extra-oral time. Saliva was selected as the best transport media for avulsed tooth by $40.1 \%$ of the participants. Regarding physician's attitude, 20\% showed interest in receiving information about the subject.

Conclusion: Dento - alveolar trauma does not pose a significant morbid risk for the trauma patient. However, failure to recognize or obtain appropriate consultation can result in premature tooth or alveolar bone loss, resulting in problematic prosthetic rehabilitation, along with immediate and long-term emotional and social impact, especially among young adults. This study revealed that the majority of ER physicians lack the knowledge needed to manage avulsions cases. Hospital emergency departments are not confronted with managing dental emergencies of both traumatic and non-traumatic origin.

Key words: Dental trauma, traumatic injuries, emergency department

\section{Dental Trauma and Its Management}

\author{
Erza VOCA MULAJ ${ }^{1}$, Fatime LENJANI ${ }^{2}$, Basri \\ LENJANI $^{2}$ \\ ${ }^{1}$ University Dentistry Pristine, Kosovo \\ ${ }^{2}$ Emergency Clinic, University Clinical Center of \\ Kosovo, Pristine, Kosovo
}

\begin{abstract}
Introduction: Traumatic dental injuries often occur as a result of an accident or sports injury. Dental injury often occurs in the context of other trauma. The majority of these injuries are minor - chipped teeth. It's less common to dislodge your tooth or have it knocked completely out but these injuries
\end{abstract}


are more severe. Assessment and management should begin with the airway, cervical spine, breathing and circulation, including control of major bleeding.

Purpose. Traumatic teeth injuries pose a challenge to dentistry. The purpose of this study is to give a classification of dental trauma based on localization, clinical and prevalence.

Material and methods; This paper is based on contemporary literature, the data obtained from the NCSCU in order to serve as theoretical and practical scientific information in dentistry.

Results and Conclusions; Based on the above objectives, they lead us to the results which consist in the very important statistical data. Studies have been selected in data at range from ages 14 to 18 years males are more at risk than females in ratio 2: 1. Frequent traumas are of the type of contusion that affects the maxillary and fracture of the tooth crown. At the end of this scientific elaboration, we reach the conclusions which are the essence of knowledge on dental trauma. Traumatic dental injuries are treated as emergencies in dentistry. Prognosis and treatment are in relations with the treatment of traumatic dental pathologies. Dental trauma management remains a problem in dental practice based on factors that complicate the treatment. Dental practitioners should have knowledge in treating and managing dental trauma.

Keywords; trauma, emergency, management.

\section{Management and Emergency Dental Approach to Periodontal Abscesses}

Erza VOCA ${ }^{1}$ MULAJ, Fatime LENJANI ${ }^{1}$, A. LENJANI, Berat LENJANI ${ }^{2}$

${ }^{1}$ University Clinical Dentistry Center of Kosovo, Pristine, Kosovo

${ }^{2}$ Stomatological Hospital Pristine, Kosovo

\section{Abstract}

Periodontal abscess is the third most frequent dental emergency, representing 7-
$14 \%$ of all dental emergencies. The sick often report to the Department of Emergencies complaining of a "toothache". Common causes of toothache are multifungal. The difference and the type of abscess can affect the treatment decisions, prognosis and sickness of the patient. The correct diagnosis and proper treatment of these diseases require that the dental emergency doctor have a basic understanding of dental anatomy, pathophysiology and dental treatment protocols. Many of these conditions can be managed initially through the Department of Dental Emergencies. The diligent dental practitioner should have a clear understanding that these infections can become very complex and may require consultations or referrals on time. Today, three therapeutic methods are being discussed in dentistry, involving, drainage and debridement, systemic antibiotics and periodontal surgical procedures that are applied in the chronic stage of the disease.

Keywords. Aspen periodontal, emergency, tooth, dental

\section{Clinical Importance of the Areas of Cortex, Functional Lateralization, Dominance and Asymmetry in the Human Brain!}

\author{
Sadi BEXHETI ${ }^{1}$, Selim ÇERKEZI ${ }^{2}$, Veton \\ ADEMI $^{3}$, Jeton SHATRI ${ }^{3}$ \\ ${ }^{1}$ Department of Anatomy, Faculty of Medicine, \\ University of Tetovo, Republic of Macedonia; \\ ${ }^{2}$ Institute of Anatomy, Faculty of Medicine, \\ University of Pristina, Republic of Kosovo; \\ ${ }^{3}$ Department of Radiology, Faculty of Medicine, \\ University of Pristina, Republic of Kosovo
}

\section{Abstract}

The aim of this article is to indicate functional localization in the areas of cerebral cortex. The cytoarchitectonic map of the adult human cerebral cortex, prepared in the beginning of the 20th century, is one of the greatest achievements in neuroscience.

Here, we want to describe all the versions of 
Brodmann's map and other authors, highlighting all the modifications made especially in the cortex areas related to the speech and language in the left cerebral hemisphere. In this paper, are described the mechanisms of functional localization in the visual cortex, auditory, motoric/premotoric area, somatosensoric and all other functions. Our results indicate about hemisphere lateralization (influence of the left and right hemisphere) and dominance that lead to microscopic and macroscopic asymmetry of the human brain. In our work, we used 100 brains that by their history of disease, were normal brains, which are fixated with formalin. Certain areas are put into celoidine patterns. After that, we used the dyeing methods of Niessl, Golgi, histochemical and imunocytochemical. We came to a conclusion that all of the areas of the cerebral cortex have their laminar and columnar cytoarchitectonic mostly dominated by the left side that lead to functional lateralization and micro and macroanatomical asymmetry. The left hemisphere, potentially capable for abstract thought, would never be able to realise its task if it would not process the inputs of the concrete thought that are provided from the right hemisphere. Functional collaboration between the two hemispheres of brain, provides the normal flow of our psychological activity as a whole.

Keywords: Cytoarchitecture, human brain mapping, lateralization, dominance, asymmetry.

\section{Emergency Treatment of Spontaneus Subarachnoid Hemorrhage}

Kiril LOZANCHE ${ }^{1}$, Jovica UGRINOVSKI ${ }^{1}$, Ljuzim AGAI ${ }^{1}$

${ }^{1}$ Clinical Hospital, Acibadem Sistina, Skopje, MACEDONIA

\section{Abstract.}

The patient with spontaneous subarachnoid hemorrhage ( $\mathrm{SAH})$ belongs to cases of the emergencies in neurosurgery.

The main cause of SAH is ruptured intracranial aneurysms with the incidence of $75-80 \%$.

The outcome of the patients with $\mathrm{SAH}$ in the literature is: $10-15 \%$ of patients die before reaching medical care. Mortality $10 \%$ within first few days. Overall mortality is $45 \%$. Rebleeding is the major cause of morbidity and mortality, the risk is $15-20 \%$ within two weeks.

Material: In the period between November 2010 to November 2017, we have operated on 39 ruptured aneurisms in acute stage. All patients with SAH were investigated within first 24 hours in the Emergency department: CT of the brain and MRI or CT angiography. Those patients with Hunt \& Hess $(\mathrm{H} \& \mathrm{H})$ grade 2 or more were admitted to ICU (monitored bed); The surgery consists of "clipping" of the aneurism neck. All of the patients were treated with hyperdynamic therapy (triple $\mathrm{H}$ therapy) and Nimodipine.

Results: 30 patients were admitted in $\mathrm{H} \& \mathrm{H}$ grade 1 or $2 ; 9$ patients with grade 3,4 or 5 . 30 patients were operated on the first 72 hours from insult ("early surgery"), 9 patients after 12-14 days from insult ("late surgery"). Poor outcome was in 3 patients.

Coclusion: Our experienced team of: neurosurgeon, anesthesiologist, neuroradiologist and other staff allow the patients prompt and safely diagnosis and treatment with this very dangerous disease.

\section{Selection of the Best Surgical Approach in the Treatment of Sub Axial Cervical Spine Fractures}

Jordan SAVESKI ${ }^{1}$, Neda TRAJKOVSKA ${ }^{1}$, Ilir HASANI $^{2}$

${ }^{1}$ Clinical Hospital Acibadem Sistina, Skopje, MACEDONIA

${ }^{2}$ University Clinic of Traumatology, Medical

Faculty, Skopje, MACEDONIA 
Abstract

Introduction: Clearance, early and correct diagnoses of cervical spine injurie (CSI) is still a common problem in traumatology especially in polytraumatized patients. Delayed or missed diagnoses leads to catastrophic consequences. The choice of the best surgical approach is still controversial. The aim of this study is to present our experience in clearance, assessment and surgical treatment of subaxial cervical spine injury (SCSI).

Material and methods: From $2000-2014$ year, $420 \mathrm{pt}$ with SCSI were treated surgically. There were $62 \%$ male and $38 \%$ female patients. Of all patients $72 \%$ were younger than 50 years and nearly $30 \%$ were in the $3^{\text {rd }}$ decade. The main cause of injury were MVA in $244 \mathrm{pt}(58 \%)$, diving in $67 \mathrm{pt}$ (16\%), fall from height $80 \mathrm{pt}(19 \%)$ and other injuries $29 \mathrm{pt}$ (7\%). Initial evaluation was done by ATLS and $\mathrm{ABC}^{\mathrm{s}}$ protocol. Primary survey included: established airway, ventilation, maintain blood pressure $>90$ and distinguish the type of shock (hemorrhagic, neurogenic or spinal) and immobilization of the spine with $\mathrm{C}$ collar and back board.

Results: Neurologic recovery was as followed: group A (total $92 \mathrm{pt}$ ), from whom $69 \mathrm{pt}$ remained in the same group , $14 \mathrm{pt}$ group B, 6 pt in group C, 2 pt in group D and $1 \mathrm{pt}$ in group E; group B (total $59 \mathrm{pt)} 9$ pt maintain in group B, $18 \mathrm{pt}$ in group C, 22 pt in group D and 10 pt group E; group $C$ (total $63 \mathrm{pt}) 11 \mathrm{pt}$ maintain in the same group, $40 \mathrm{pt}$ in group D and $12 \mathrm{pt}$ in group $\mathrm{E}$; group D (total $47 \mathrm{pt}$ ) $21 \mathrm{pt}$ maintain in group D and $46 \mathrm{pt}$ in group E; group $\mathrm{E}$, all of them maintained in the same level group E. Initial improvement in the alignment of deformities was achieved in all patients. Nine pt were re-operated (because of incomplete reduction, 6 of them primary treated in another institution). Eleven pt had postoperative infection (10 pt superficial and 1 pt deep infection).

Conclusion: Definitive surgical treatment was determined by the type of injury, severity of instability and neurological status. Both anterior and posterior fixation appear to be adequate. Combined approach was indicated in patient with marked instability.

\section{A Prospective Study for Operative Treatment of Spine Fractures at the Clinic of Traumatology, Skopje, in the Period From 2014 to 2017; Evaluation, Surgical Approach, Follow ups and Functional Outcomes}

\section{Simon TRPESKI ${ }^{1}$ \\ ${ }^{1}$ University Clinic of TOARILUC, Ss. Cyril and Methodius University-Medical faculty, Skopje, Macedonia.}

\section{Abstract}

Introduction: Fractures of the spine represent a common pathology. They are represented by $6 \%$ of all fractures of the skeleton and thoracic-lumbar fractures with $90 \%$ of the spine fractures. In $20 \%$ of patients with this type of injury there are neurological outbreaks on the first examination in the emergency room. Causes are car accidents (45\%), falls (20\%), sports $(15 \%)$, acts of violence $(15 \%)$, and miscellaneous activities $(5 \%)$ are the primary causes of spinal fractures.

Materials and methods; At the Clinic of Traumatology in the period from 2014 to 2017, a prospective study was conducted for patients with fractures of the spine. In this study, 157 patients were evaluated after a trauma event $(\mathrm{m}: \mathrm{f}=102: 55)$, at a median age of 58 years, all of them operatively treated. All patients were subjected to appropriate radiological investigations, AP and lateral views of the spine, CT and MRI of the spine.

Results: From 157 patients, complications occurred in 40 patients, 12 patients had decubitus wounds of second degree, and 9 dehiscence of the operation wound, in 6 patients urinary tract infections occurred, and 22 patients had complications as a result from the polytrauma. In a complete spinal cord injury (Frankle A) in 12 patients, despite surgery, the changes were definitive. In these patients, the occurrence 
of contractures was common. In patients with complete outbreaks of motility,

\section{Conclusion}

The treatment of fractures of spine is a complex process that requires great engagement, education and experience and watchfulness by the responsible surgeon.

Keyword: Fractures of the spine, Frankle classification...

\section{Brain Heart Interaction in Head Injury}

Ayman El-MENYAR ${ }^{1}$

${ }^{1}$ Director of clinical Research in Trauma and vascular surgery in Hamad General Hospital, Doha, Qatar

\begin{abstract}
Diagnosis of cardiac injury is easily overlooked in trauma patients. The concept of brain-heart interaction is an interesting research field. The neurocardiac axis theory and neurogenic stunned myocardium phenomenon could partly explain the brainheart link. Several observational retrospective studies have demonstrated worse outcomes in head injury patients who had positive serum troponin as well. Also, studies have shown a favorable effect of beta-adrenergic blockers in patients with traumatic brain injury in terms of reducing the overall TBI-related mortality. However, several questions remain to be answered in the prospective clinical trials.
\end{abstract}

\section{Brain Tumors after Brain Trauma}

Blerim ZEQIRI ${ }^{1}$, Mentor PETRELA ${ }^{2}$

${ }^{I}$ Neurosurgery Department, Clinic Hospital of

Tetovo, MACEDONIA

${ }^{2}$ Neurosurgery Department, University Hospital

Center "Mother Teresa", Tirana, Albania

\section{Abstract}

The main purpose of this paper is to present: diagnosis, clinical reflection, differential diagnosis, complications and treatment of head tumors as a consequence of trauma

Material and methods; Data on the diagnosis of head traumas in children were collected from patient records and operational data at the Department of Neurosurgery, Clinical Hospital Tetovo, including the period 01.01.201831.08.2018. The data were analyzed based on age, sex and type of tumor as a result of head trauma.

Results; In the Department of Neurosurgery at the Tetovo Clinical Hospital there were 2167 cases with head trauma for the period January 2018 to August 31, 2018 of all age groups. From 0 to 14 years old are 67 cases Out of the total number of over $3.5 \%$ of the visits are head trauma, aged 0 to 14 years of whom.

Conclusion: Head traumas are $35.0 \%$ of the incidence of diseases caused in the Tetovo region, the highest probability of trauma in males is $65 \%$, while women in females is $35 \%$ lower. In most cases it is biased, but bilateral cases are not excluded.

Key words: Tumors, Trauma, Head, Child.

\section{Current Management of Necrotizing Soft Tissue (NSTI); Timing of Debridement is everything.}

\section{Rifat LATIFI ${ }^{1}$}

The Felicien Steichen Professor and Chairman of Surgery. New York Medical College, School of Medicine. Director, Department of Surgery, Westchester Health Network. Department of Surgery, New York Medical College

\begin{abstract}
NSTIs, in particular, necrotizing fasciitis, remain potentially the deadliest form of surgical infections and require aggressive resuscitation and surgical debridement. Early diagnosis, early antibiotic treatment, and early surgical debridement remain the cornerstone of care for these patients. Yet, timely recognition of NSTIs is difficult, but their clinical presentation is of key importance. Patients who are thought to possibly have NSTIs must be seen, as soon as feasible, by the most experienced surgeons. Once NSTIs are diagnosed, the proper interventions must be conducted immediately. Emergent, timely surgical debridement remains crucial. Early surgery
\end{abstract}


(within the first $6 \mathrm{~h}$ after diagnosis) improves hospital outcomes in patients with severe NSTIs.

Early recognition of NSTIs is difficult, but their clinical presentation is of key importance. Patients who are thought to possibly have NSTIs must be seen, as soon as feasible, by the most experienced surgeons. Once NSTIs are diagnosed, the proper interventions must be conducted immediately. Emergent, timely surgical debridement remains crucial. Early surgery (within the first $6 \mathrm{~h}$ after diagnosis) improves hospital outcomes in patients with severe NSTIs. Further prospective studies are warranted to support these findings. In this paper I will outline the current approaches to NSTI.

\section{Gastric adenocarcinoma advanced in}

\section{a final year student. A case report}

Imri VISHI, Ragip BYTYQI, Sami SPAHIU, Nijazi VISHI,

Department of Surgery, Regional Hospital, Ferizaj, KOSOVO

\section{Abstract. \\ Gastric carcinoma is most common in the $50-70$ age group [2], and is extremely rare under the age of 30; grows rapidly and steadily to reach higher levels in older age groups [3]. Basically, there are two sub- types of adenocarcinoma arising from the gastric mucosa: intestinal and diffuse based on Lauren (1965) criteria.}

The disease was fatigue, with dysphagia associated with solid food, with anorexia, and with weight loss; (about $10 \mathrm{~kg}$ for the last 3 months). During this period, he received symptomatic medication but without any medical visit.

Clinical examination revealed a pale skin, with decreased turgor, and in the abdomen was found a tangible mass in the abdomen, easily sensible, other quadrats of the abdomen loose...

Conclusion: Whenever symptoms of dysphagia, loss of appetite and hematemesis appear, and further investigations should be made, as early diagnosis of a gastric tumor in this group plays a crucial role in determining the prognosis.

Keywords:Gastric carcinoma, hematemesis, gastrointestinal endoscopy,

\section{Current Management of Abdominal Sepsis by the Open Abdomen Method.}

\author{
Boris E. SAKAKUSHEV ${ }^{1}$ \\ ${ }^{1}$ Medical University Plovdiv Chair of Propaedeutic \\ of Surgical Diseases, First Clinic of General \\ Surgery University Hospital St George Plovdiv, \\ Plovdiv, BULGARIA
}

\section{Abstract}

Since the beginning of this century the method of Open Abdomen treatment has been approved as evidence-based lifesaving approach for abdominal sepsis.

Open Abdomen treatment is indicated in severe sepsis or septic shock/ and prevention of Abdominal Compartment Syndrome. Rezende-Neto et al. already have defined that there are three basic indications for Open Abdomen anatomical, physiological and logistical.

The main objectives of the Open Abdomen approach both in severe secondary peritonitis and severe acute pancreatitis are sepsis control and expedite subsequent surgical interventions.

"Planned" or "on demand" re-laparotomy for Open Abdomen must be done early, most appropriately until the 48th hour.

Following re-exploration, we focus on early and definitive closure of the abdomen, in order to prevent and reduce complications like entero-atmospheric fistulas, fascial retraction with loss of abdominal wall domain, development of frozen/stiff/ abdomen and massive incisional hernias.

Intra-abdominal operations/manipulations in abbreviated re-laparotomy include general intra-abdominal procedures aspiration, lavage, necrectomy and specific ones - suture, resection, anastomosis, stoma which respond the basic objectives of Open Abdomen - a) determining the cause of peritonitis; b) draining fluid collections; c) 
controlling the origin of the abdominal sepsis".

There is no consensus for the optimal type of temporary abdominal closure technique, although some benefits of constant negative pressure devices recently are advocated. temporary abdominal closure using negative pressure therapy should ideally allow the abdomen to be closeed as soon as possible within 1 week.

The best mode of restitution of the abdominal wall is progressive closure and complete adoption of the fascia defect by primary closure if possible till the 8th day of inndex operation

The most serious local complication of Open Abdomen - entero-atmospheric fistula often requires surgical treatment, depending on site and size.

Although there is an increasing evidence for rising rate of life threatening complications following Open Abdomen treatment, implying its limitation, it is still method of choice in severe abdominal sepsis and septic shock.

Keywords: Open Abdomen, Abdominal Compartment Syndrome, Temporary Abdominal Closure, Negative Pressure Therapy

\section{Timing of Cholecystectomy in Cases of Biliary Pancreatitis}

Orhan ALIMOGLU ${ }^{1}$

${ }^{1}$ Department of General Surgery, School of Medicine, Istanbul Medeniyet University, İstanbul, TURKEY

\begin{abstract}
Biliary calculi (stone, microlithiasis, sludge) are the leading cause of acute biliary pancreatitis (ABP). The pathogenesis of ABP involves a temporary obstruction of the ampulla of Vater by a biliary calculus, causing bile reflux into the pancreas via a common channel; or the passage of a stone leading to the temporary opening of the sphincter allowing regurgitation from the duodenum into the pancreatic duct.
\end{abstract}

For the treatment of ABP, although clearance of the common bile duct and gallbladder removal are generally accepted methods for decades, propertiming of the intervention is still controversial. For cholecystectomy there are two approaches that concern propertiming:

(1) interval cholecystectomy: postponing cholecystectomy for $6-8$ weeks may reduce the acute inflammation, making it easier to perform laparoscopic cholecystectomy and possibly lowering the conversion rate;

(2) delayed cholecystectomy (48 hours after admission): performing cholecystectomy during the first admission after clinical improvement may reduce the incidence of recurrent attacks of ABP, morbidity, and hospital expenses.

Definitive treatment of mild to moderately severe ABP can be accomplished effectively and safely via cholecystectomy following clinical improvement and normalized levels of laboratory parameters during the first admission. Waiting for $6-8$ weeks to perform cholecystectomy may result in recurrent biliary pancreatitis (upto $60 \%$ ), which may increase morbidity and the length of the hospital stay. On the other hand, in cases of severe ABP, interval cholecystectomy may be the preferred choice in order to diminish possible complications and morbidity.

Conclusions: The timing of cholecystectomy in ABP depends of the severity of the diseases and the clinical course of the individual patient.

\section{Surgery for Acute Cholecystitis: When?}

\author{
Ozgur EKINCI ${ }^{1}$ \\ ${ }^{1}$ Department of Surgery, School of Medicine, \\ Istanbul Medeniyet University, Istanbul, Turkey
}


hospitalized with a first line treatment including I/V fluids, antibiotics, and analgesia. Ultimately, surgery remains to be the only definitive therapeutic approach for patients having been diagnosed with acute cholecystitis, and laparoscopic cholecystectomy is the preferred surgical technique. However, in the past and at present, the timing of cholecystectomy has been a matter of debate.

"Early cholecystectomy" performed within 96 hours of the illness is preferred over "interval or delayed cholecystectomy" that is performed 6-10 weeks after initial medical treatment and recovery. Current scientific evidence favour early cholecystectomy over the delayed approach, unless the patient is unfit for surgery, as this approach possesses the benefits of adefinitivesolution in onehospitaladmission,

quickerrecoverytimes, and earlier return to work. Although most surgeons prefer to perform early cholecystectomy within 96 hours from the onset of symptoms, the interval approach is still being used for the management such cases. On the other hand, for cases having admitted after 96 hours from the onset of symptoms, interval cholecystectomy is preferred, yet some surgeons still choose the option of early surgical approach. This presentation aims to discuss the surgical approach for in cases of acute cholecystitis.

\section{The Treatment of Patients with Cholelithiasis.}

Skender VELIU ${ }^{1}$, Jasmina KRÖPFL ${ }^{2}$, Dominika VRBNJAK ${ }^{3}$

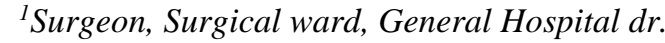
JožePotrč Ptuj, Potrčevacesta 23, 2250 Ptuj, Slovenia

${ }^{2}$ Nurse, General Hospital dr. JožePotrč Ptuj, Potrčevacesta 23, 2250 Ptuj, Slovenia

${ }^{3}$ University of Maribor Faculty of Health Sciences, Žitnaulica 15, 2000 Maribor, Slovenia

\section{Abstract}

Introduction: In the recent years cholelithiasis has become one of the most common conditions affecting the digestive system world-wide. It affects approximately $1 / 10$ of the adult population, with the higher incidence in women. Symptoms are present only in $20-30 \%$ of the affected population; in other cases the disease is asymptomatic. In this article we present the causes, symptoms, diagnostic tools available for screening as well as the current treatment guidelines for cholelithiasis. The reasons for gallstone formation are obesity, increased levels of cholesterol, increased age and certain drugs especially oral contraceptives. The most common symptoms are pain in the right upper abdomen and in the epigastric area. The diagnosis is made based on the clinical presentation, laboratory findings and ultrasound of the abdomen. Current guidelines for conservative and surgical management of cholelithiasis are presented in this article with the emphasis on laparoscopic cholecystectomy which is the golden standard for the treatment of holelithiasis. We present the statistical figures and results for operative treatment of patients with cholelithiasis at General Hospital Dr. Jože Potrč Ptuj, Slovenia for the period of 2013-2017.

Keywords: Cholelithiasis, conservative treatment, surgical treatment, laparoscopic cholecystectomy, guidelines

\section{Prophylactic Use of Antibiotics in the Intensive Care Unit}

Hektor SULA ${ }^{1}$, Rudin DOMI ${ }^{1}$

${ }^{I}$ Department of Anesthesia \& Intensive Care,

${ }^{1}$ University of Medicine, Tirana, Albania

\begin{abstract}
The appropriate use of antibiotics in ICU is important in ensuring an optimal clinical outcome, but also in controlling the emergence of resistance among pathogenic microorganisms and in containing costs The polyvalent ICU contains heterogeneous patients under therapeutic treatment and prophylactic antibiotics in order to minimize the infection. The importance of the prevention of infection in critically ill patients is therefore based on its potential to
\end{abstract}


reduce both morbidity and mortality. A clear distinction should be made between surgical and non-surgical patients.

The antibiotic prophylaxis (ABP) in emergency surgery seems to be difficult compared to elected procedures. Sometimes the difference between prophylaxis and early treatment is difficult to be done.

Risk factors for ICU infection: The frequent device utilization in intensive care is responsible for most nosocomial ICUacquired infections. Endotracheal intubation, mechanical ventilation, central venous catheter, and urinary catheterization are most important procedures responsible for the infections of respiratory tract, bloodstream, and urinary tract in ICU patients. The first week of ICU admission is characterized by impaired host defense (especially neutropenic patients), contributing in increased infection during these procedures. The early use of antibiotics is able to prevent the infection in these situations.

Antibioprophylaxis strategy: Gastrointestinal decontamination. Systemic Prophylaxis. Preoperative Antimicrobial Prophylaxis

Common Misuses of Antibiotics: Prolonged Empiric Antimicrobial Treatment without clear evidence of infection. Prolonged Prophylactic Therapy. Excessive Use of Certain Antimicrobial Agents. Resistance to chlorhexidine

Conclusions; Use of antibiotics for prophylactic purpose in ICU is an important maneuver, which needs to be taking into consideration strict indicators, patient category kind of ABP, application modality, time length, possible complications and economic factors (costs).

\section{Extra Corporal Therapies in Sepsis.}

Alfred IBRAHIMI ${ }^{1}$

${ }^{I}$ Anesthesia and Intensive Care Service University Hospital Center "Mother Theresa"-Tirana, Albania.
The treatment of sepsis is an ongoing challenge for clinicians; despite the wide choice of effective antibiotics to treat infection, sepsis remains the leading cause of morbidity and mortality for patients admitted to an intensive care unit. Dysregulation of the immune response is now recognized to be a key factor in multiple organ dysfunction, yet our therapy for inflammation remains ineffective. It has been advocated for more than a decade that cytokine reduction in blood compartment could lead to a reduction in mortality in sepsis. Over the years, multiple extracorporeal techniques have evolved, with the intent of influencing the circulating levels of inflammatory mediators like cytokines and chemokines, the complement system, as well as factors of the coagulation system. These include high-volume hemofiltration, use of high cutoff membranes, and systems based on adsorption, such as coupled plasma filtration adsorption and the polymyxin - B column. In addition, new experimental systems that utilize human phagocytic cells and immobilized antibodies for targeted immunomodulation have emerged. In the context of limited resources and growing expansion in the availability of technologies, a better understanding of these therapies is required before they can be properly integrated into standard clinical practice in the hope of influencing major clinical outcomes.

Keywords: sepsis, therapy, extracorporal

\section{Bleeding Complications after Urgent first time CABG: Impact of Clopidogrel Loading for Coronarography}

\author{
Aleksander HOXHA ${ }^{1}$, Sokol SHEHU, Rezar \\ DEVEJA, Thoma QIRJAZI ${ }^{2}$, Shkelqim \\ MET'HASANI ${ }^{1}$, Merita ZEKA \\ ${ }^{l}$ Department of Anesthesia \& Critical Care, \\ ${ }^{2}$ Medical University of Tirana, Albania \\ Hygeia Hospital, Tirana, Albania
}

\section{Abstract}

\section{Abstract}


This study was designed to evaluate the impact of preoperative clopidogrel on bleeding and outcomes after coronary artery bypass graft surgery (CABG).

Background. Excessive bleeding impairs outcome after coronary artery bypass grafting (CABG). Clopidogrel in combination with aspirin, given before percutaneous coronary intervention, have become the standard for stent thrombosis prevention. Some premedicated patients, however, are found to need surgical treatment, thus platelet inhibition caused by clopidogrel becomes a concern for post operation major bleeding.

Methods. The study represent a observational retrospective analysis of collected data. The follow up of 223 treated with clopidogrel and aspirine and 77 patients not receiving treatment with platelet antagonist 7 days before $\mathrm{CABG}$ are analysed.

Results. The groups were comparable in age, gender, body surface area, preoperative hematocrit, preoperative prothrombin time and prior myocardial infarction. The clopidogrel group had higher12h and $24 \mathrm{~h}$ mean chest tube output (at $12 \mathrm{~h}$ mean $519.7 \mathrm{ml}$ vs $353.1 \mathrm{ml}, \mathrm{p}<0.05$, at $24 \mathrm{~h}$ mean $756.6 \mathrm{ml}$ vs $563.5 \mathrm{ml}, \mathrm{p}<0.05)$. Moreover, reoperation for bleeding was 4.5 -fold higher in the clopidogrel group (5.9\% vs. $1.3 \%$, p $<0.01$ ), and more transfusions of red blood cells (3.23U vs $2.6 \mathrm{U}, \mathrm{p}<0.05)$, platelets $(1.53 \mathrm{U}$ vs $1.23 \mathrm{U}, \mathrm{p}<0.01)$ and fresh frozen plasma $(0.84 \mathrm{U}$ vs $0.36 \mathrm{U}, \mathrm{p}<0.01)$. The clopidogrel group also showed a longer mechanical ventilation time (16.9h vs 12.9 $\mathrm{h} \mathrm{p}=0.03$ ) and trend towards more prolonged stay in ICU (2.08 days vs 1.7 days $\mathrm{p}=0.048$ ).

Conclusions. Clopidogrel in combination with aspirin before CABG is associated with higher postoperative bleeding, exposure to blood products and morbidity. These findings raise concern regarding the routine administration of clopidogrel before anticipated but undecided coronary stent implantation.

Keywords: Bleeding complications, CABG, Clopidogrel

\section{Burns in Elderly Patients. New \\ Approaches in Treatment of Combustional Shock Phase. A case of a Survived Old Patient with $40 \%$ of TBSA.}

\author{
Albana ALEKSI ${ }^{1}$ \\ ${ }^{I}$ The Service of Burns and Plastic Surgery, \\ University Hospital Center "Mother Teresa", \\ Tirana, Albania.
}

\begin{abstract}
Introduction: Nowadays many old people live and stay home alone, because the young people go abroad.

The old burned patients represent a growing number in the total burned patient number in our emergency. This segment of population presents different features and their survivalfrom the state of burn depends from the surface and the grade of damage, the co-morbities and an accurate treatment to prevent the complications and to improve the outcomes.

The Case; A male patient 68 -yr old was admitted to our emergency room after he accidentally found himself within the lime pit. The place of accident was $35 \mathrm{~km}$ from the burn center. No venous access. No medication. No medical treatment.
\end{abstract}

The region affected were the lower extremities, the low back and the upper extremities (half of them). After washing the wounds with $\mathrm{NaCl} 0.9 \%$, we medically treated them with petrolatum gauze and Betadini $7.5 \%$ and the patient was transferred in intensive care. The patient was fully monitored with central venous pressure, arterial, urinary catheterization. The patient suffers from arterial hypertension and in the last two years he was subjected a coronary bypass. No signs of initial necrotomia and the compartment syndrome. Our attention was paid to rehydrate the patient as good as possible to avoid the overloading because he was a cardiac patient. The fluid resuscitation was administered guided by Parkland formula i.e $4 \mathrm{ml} / \mathrm{kg} / \%$ TBSA in order to maintain a urine output $0.5-1 \mathrm{ml} / \mathrm{kg} /$ hour. We gave him Ringer lactate and after 12 hours 
post combustio, we gave him colloid solution (human albumine 20\%-50 ml and 2 fresh frozen plazma izogroupizoRhesus). Keywords: burned patients, initial necrotomia, petrolatum gauze

\section{Thoracotomy and Postoperative Pain Management}

\author{
Alma CANI ${ }^{1}$, Fahri KOKICุI ${ }^{1}$, E. SHIMA ${ }^{1}$, Fadil \\ GRADICA $^{2}$, L. AGOLLI ${ }^{1}$ \\ ${ }^{1}$ Anesthesiology Service, University Hospital \\ "Shefqet Ndroqi" Tirana, Albania \\ ${ }^{2}$ Thoracic Surgery Service, University Hospital \\ "Shefqet Ndroqi" Tirana, Albania \\ 1,2 Pneumology Service, University Hospital \\ "Shefqet Ndroqi" Tirana, Albania
}

\begin{abstract}
Background: Post thoracotomy pain syndrome (PTPS) is unfortunately very common following thoracotomy and results in decreased quality of life. Treatment of acute post-thoracotomy pain is particularly important not only to keep the patient comfortable but also to minimize pulmonary complications.
\end{abstract}

The aim of this retrospective study was to determine perioperative patient, surgical, and analgesic characteristics associated with the development of PTPS.

Materials and Methods: In a retrospective study, 163 patients were randomized to receive multimodal analgesia including opioids and non-opioids, delivered through various routes, neuraxial use of local anesthetics, either alone or in combination with other drugs, nerve blocks, antihyperalgesics and techniques such as patient controlled analgesia, prevertebral or epidural infusion for post-thoracotomy pain control. Patients were evaluated for analgesic efficacy and postoperative complications

Results: Incidence of long-term postthoracotomy pain has been reported to be $80 \%$ at 3 months, $20 \%$ at 6 months, and $5 \%$ at one year after surgery; incidence of severe pain is $3-5 \%$, and pain that interferes with normal life is reported by about $25 \%$ of patients, $5 \%$ of the patients with PTPS received treatment for pain. The strongest predictive factors for the development of Pulmonary Postoperative Complication CPP were: thoracic pain for three months post operatively (odds ratio $[\mathrm{OR}]=3.54$, $95 \%$ confidence interval $[\mathrm{CI}]=1.69$ 7.40, $\mathrm{P}=0.001)$, thoracic pain for 12 months postoperatively $(\mathrm{OR}=2.73,95 \%$ $\mathrm{CI}=1.28-5.83, \quad \mathrm{P}=0.009) \quad$ Neuropathic pain was present in $4.8 \%$ of patients. Urinary retention, however, was noted to be significantly more frequent in patients with epidural catheter.

Conclusions: The introduction of multimodal analgesia including opioids and non-opioids, delivered through various routes, neuraxial use of local anesthetics, either alone or in combination with other drugs, nerve blocks, anti hyperalgesics and techniques such as patient controlled analgesia and pre-emptive analgesia have greatly improved the efficacy of paincontrol while minimizing the side-effects of any one modality. The recent recommendation of planning the pain services in an organized manner and implementation of Acute Pain Services (APS) has proven to be beneficial and rewarding.

Keywords: thoracotomy, pain, management

\section{Fluid Management in Traumatic Brain Injury and Neurological Patient}

Asead ABDYLI ${ }^{1}$, Blerim ARAPI ${ }^{1}$, Gentian HUTI ${ }^{1}$, Rudin DOMI ${ }^{1}$.

${ }^{1}$ Anesthesiology and Intensive Care Service,

American Hospital Tirana, Albania

\section{Abstract}

Introduction; Maintenance fluids in critically ill brain-injured patients are part of routine critical care. Both the amounts of fluid volumes infused and the type and tonicity of maintenance fluids are relevant in understanding the impact of fluids on the pathophysiology of secondary brain injuries in these patients. In this narrative review, current evidence on routine fluid management of critically ill brain-injured 
patients and use of hemodynamic monitoring is summarized. Pertinent guidelines and consensus statements on fluid management for brain-injured patients are highlighted. In general, existing guidelines indicate that fluid management in these neuro critical care patients should be targeted at euvolemia using isotonic fluids. A critical appraisal is made of the available literature regarding the appropriate amount of fluids, hemodynamic monitoring and which types of fluids should be administered or avoided and a practical approach to fluid management is elaborated. Although hypovolemia is bound to contribute to secondary brain injury, some more recent data have emerged indicating the potential risks of fluid overload. However, it is acknowledged that many factors govern the relationship between fluid management and cerebral blood flow and oxygenation and more research seems warranted to optimise fluid management and improve outcomes.

Keywords: Subarachnoid haemorrhage, Traumatic brain injury, Fluid management, Volume status, Hypervolemia, Haemodynamic monitoring

\section{Crush Syndrome and Continuous Renal Replacement Therapy (CRRT)}

Rinard KORTOCI ${ }^{1}$, Blerim ARAPI ${ }^{1}$

${ }^{I}$ Anesthesiology and Intensive Care Service, American Hospital Tirana, ALBANIA

\section{Abstract; \\ Introduction; Mioglobin is a protein based on iron and enables $\mathrm{O} 2$ to be transported to the muscles. In cases of rabdomiolize (Crush Syndrome) due to muscle damage, the myoglobin is thought to be the main reason for the IRA (through some mechanisms already confirmed).}

Purpose. Evaluation of IRA treatment modalities in rhabdomyolysis by focusing mainly on CRRT as well as our experience in treating a serious septic patient of IRA (Anorexia).
Discussion. In addition to conventional treatment in Crush Syndrome, CRRT (CVVH and SHF), hemodialysis, are also of great importance. The starting point of CRRT depends on IRA and / or MODS and not on myoglobinemia or LDH level. In 2014 there have been reports of the use of SHF (super high flow hemofiltration) which is carried out with filters with larger pore sizes than ordinary filters.

Keyword; Crush syndrome, IRA, CRRT, rabdomiolize, myoglonine, SHF

\section{Management of a Iatrogenic Complete Obstruction of the Inferior Vena Cava with CRRT Technique}

Saimir KUCI ${ }^{1}$, Alfred IBRAHIMI ${ }^{1}$, Ervin BEJKO ${ }^{1}$, Stavri LLAZO ${ }^{1}$, Jonela BURIMI ${ }^{1}$, Esmerilda BULKU ${ }^{1}$.

${ }^{1}$ Anesthesia and Intensive Care Service University Hospital Center "Mother Theresa"-Tirana, ALBANIA.

\section{Abstract}

Introduction: Complete obstruction of the inferior vena cava is a deadly complication. Herein we represent a case with a iatrogenic complete obstruction of the inferior vena cava treated in the intensive care employing a bypass between the left subclavian and left femoral veins.

Case presentation. A 68-year-old male patient presented an abdominal aortic aneurysm $(6 \mathrm{~cm})$. He underwent abdominal aortic aneurysm repair. During the operation, the inferior vena cava was accidentally perforated. Given the emergency of this life-threatening condition, it was impossible for the surgical team to stitch the perforation and correct the complication. Inferior vena cava was ligated in order to stop the bleeding. Complete obstruction of the venous flow found our patient's collateral veins unprepared, and therefore unable to perform adequate drainage of the blood from the lower extremities, resulting in inadequate preload and the following hypovolemic shock. We found a practical, off-protocol 
solution to this critical case. We inserted central venous catheters in both (left) subclavian and (left) femoral veins and connected the two with a simple perfusion tube. The gradient already present, between inferior and superior vena cava made possible a successful transfer of the blood and a good increase of the pre-load. Clinical results were almost immediate. Meanwhile the collateral veins had time to develop a greater capacity and compensate the venous obstruction.

Conclusion: We found such a bypass a very good temporary solution of this deadly complication and we believe that can be employed in other similar cases.

\section{Helicopter, Excellence Service of Medical Emergency}

Skender BRATAJ ${ }^{1}$, Erald XHELILI ${ }^{1}$, Indrit JAUPAJ $^{1}$

${ }^{1}$ National Emergency Medical Centre, Tirana, Albania.

\section{Abstract.}

Introduction: Helicopter medical emergency service is the best option to cope with emergency medical emergencies and in areas without infrastructure. Modernization and completion, not only because of modern medical equipment and devices, but more because of legal adaptation and new mentality, where every Albanian or foreign patient in the territory of the Republic of Albania is guaranteed life and treated with the same devotion, devotion and professionalism.

This service is already equipped with onboard medical equipment that is needed to monitor, defibrillate, immobilize and treat patients at the scene, during transport, to more specialized health centers. The novelty of this topic is about introducing rigorously prepared medical staff to deal with the variety of problems encountered during lifesaving operations.

The purpose of this study is to reflect and analyse the work done so far by the NCCP regarding the service, the time of arrival, the difficulties and the problems encountered during the work.

Accurate data analysis, correct documentation of events and missions through the patient's file, receiving predeparture information and completing the territorial card are essential for assessing the quality of the service, improving it, and reducing the disability of the patient. The Coordination Unit as an Innovation has a very important role in conducting this service.

As a Conclusion: Emergency helicopter service for the natural, structural conditions of our country is indispensable for life saving, service improvement, and reduction of disability.

The current state of affairs aims to improve this service to be as quality in line with the standards of international scientific associations.

Keywords: helicopter; patient's file, followup

\section{Modern Triage in the Emergency Department}

Basri LENJANI ${ }^{1}$, Gani SHABANI ${ }^{1}$, Esen UYSAL $^{1}$, Arber DEMIRI ${ }^{2}$, Erza Voca MULAJ ${ }^{2}$, Dardan LENJANI ${ }^{2}$

${ }^{I}$ Emergency Clinic, University Clinical Center of Kosovo, Pristin, Kosovo.

${ }^{2}$ University Clinical Center of Kosovo, Pristin, Kosovo.

\begin{abstract}
Background: Triage is the process of determining the priority of treatments for the sick, injured and poisoned in minor incidences and to a large extent, based on the weight and nature of their illness. Triage system. No uniform triage system exists in the United States (US), this creates a wide dilemma of basic assessment of the injured patients and sick patients and each region has its own emergency medical service network.
\end{abstract} Aim: The aim of this study is to identify contemporary triad instruments, evaluation criteria, validity and reliability reducing 
the overpopulation of the Emergency Clinic

Materials and Methods: The data were obtained from the SHSHUK-UCCK

Emergency Clinic's resources for the sixmonth period January-July 2018. Also, was taken the criteria of assessment and the examination of triad literature in the search for the adaptive model for our country in the three levels of medical care. Formation of methods that integrate the field and needs of hospitals will support EMS that ensures better care continuity. Results: Emergency Departments around the world use multiple triad systems to assess the severity, categorization of the sick and the injured. Our research identified adaptable sources and experiences of the Canadian, Australian, Finish, German, and TRI triads of the UK, which systems, resources, can impact on reducing morbidity, invalidity and mortality and affecting the level of growth of reliability in the system.

Conclusions: To regulate the current situation of the triage system in Kosovo which functions as an inadequate synchronous system or does not have any official document with standards, normative for triage at state level to develop standards, of triage norms one of the five adaptable triad system models, but adapting to our circumstances and conditions.

Keywords: Triage, system, emergency, ill, hurt criteria, assessment, and medical care.

\section{Emergency, Legislation, Ethics and Medical Deontology}

Eda LUZAJ ${ }^{1}$, Skender BRATAJ ${ }^{1}$, Ertugilda HOXHAJ $^{1}$

${ }^{1}$ National Emergency Medical Center, Tirana Albania.

\section{Abstract}

Introduction: Over the last few years, emergency medicine has grown and impressive changes. Continued medical knowledge and the introduction of technology in this field have improved the delivery of first aid in case of medical emergencies or catastrophe. The Emergency Medical Service, is responsible for any Albanian or foreign citizen who is in the territory of the Republic of Albania in case of medical assistance.

Purpose: Evidenting of ethical and legal problems at Emergency Medical Service, the way of resolving them based on Albanian legislation and what needs to be improved!

Medical staff faces patients who refuse medical care, which pose a hazard as in the case of psychiatric, alcoholic or drug users. They also face severe cases: traumatic, cardio-vascular, respiratory and tumor patients at the terminal stage. In all these cases medical personnel are involved in deontological, ethical and legal issues. Of particular importance is the preparation, completion and interpretation of the legal basis to regulate not only the medicalpatient relationship from the health but also the ethical - professional deontologic ones (to fill the legal vacuum regarding medical violations and relevant disciplinary measures).

Conclusions and proposals: Adaptation of Albanian legislation to European legislation. The term "negligent medication" Article 96 of the Criminal Code of the Republic of Albania is transformed into "neglect in diagnosis and medical treatment", thus using the same European terminology and introducing at the same time the concept of diagnosis which previously did not exist by allowing negligence in diagnosis not to be considered a criminal offense.

\section{Nursing Role in Emergency}

Edison NDREKA ${ }^{1}$, Skender BRATAJ ${ }^{1}$, Gresjeta VATA $^{1}$, Hava VANGJELI ${ }^{1}$

${ }^{I}$ National Emergency Medical Centre, Tirana, Albania.

\section{Abstract}


Introduction; Medical and nursing staff such as first aid providers are the healthcare chain (pre-hospital and hospital care).

Purpose: To identify the ethical problems in the nursing service, their role in the emergency as well as the differences and similarities between Albanian Nurse Order and Albanian Medical Order

In Law no. 147/2014 "On the Emergency Medical Service", article 20 mentions the tasks of the emergency nursing staff who are: to take care of emergency patients, depending on the sector (primary, triage reception, assessment-treatment, observation) in all their management roles and work in groups; Accurately implement emergency service protocols approved by the ministry responsible for health; to carry out all duties assigned by the Chief of Service, Head Nurse and Medical Staff; to participate in the activities for raising the technical-professional level in order to increase the quality of patient care. Increasing their role in the health service has added to the various issues related to their profession.

Ensuring standards to provide a professional, ethical and deontological service is regulated by the Statute Albanian Nurse Order and Albanian Medical Order, the Code of Ethics and Deontology for Nurses, the Law No.147 / 2014 "On the Emergency Medical Service", the Criminal Code and Civil of the Republic of Albania.

Conclusions; Improving law, ethics and deontology between the Nurses Order and the Order of Doctors. Drafting and implementing a common protocol on group work among medical staff and emergency assistants.

Competencies and duties of the nurse in emergency without the presence of a doctor!

\section{Medical Phone Triage and Ways of Organizing in the Pre-Hospital Emergency Coordination Unit.}

Skender BRATAJ ${ }^{1}$, Indrit JAUPAJ ${ }^{1}$

${ }^{I}$ National Emergency Medical Centre Tirana Albania

\section{Abstract}

Several years of analysis and experience of the medical emergency system have shown some problems: Inability to receive all calls for medical assistance, lack of triage, lack of team and ambulance control in real time via the GPS system, arrival time unobserved and very long due to traffic and location at the same ambulance site, lack of territorial card, lack of communication with the waiting emergency causing delays in handing over the patient, assessing the severity of the disease and receiving the service.

This work is a new, efficient, fast way of organizing, as one of the best European experiences that comes to the aid of the medical staff of the pre-hospital emergency service and serves as a unification of the entire triage system (Selection).

This study is based on the data collected during the last 16 months of the Coordinating Unit of the National Centre for Medical Emergencies.

As a conclusion we can say that this study highlights the quality of the antecedent system evaluation with the establishment of the National Emergency Centre, the current difficulties faced by the pre-hospital service, answering and solving all the requests for assistance, as a result brings reducing unnecessary flux and waiting in hospital emergencies; activating and tracking of available resources in real time, significantly reducing the time of arrival at the patient...

This study gives us the opportunity to shed some light on future challenges for further service improvements, more efficient use of resources and resources, better co-operation between the primary care system and primary, secondary and tertiary hospital systems, other co-operatives (firefighters, police, army in case of emergency), organizations and NGOs. All this for a single purpose: life-saving, reducing invalidity and guaranteeing the quality of life.

Key words: Medical Emergencies, Dispatch Life Support, National Emergency Centre 


\section{Current Trends in the Clinical Management of Renal Trauma}

Skender SAIDI ${ }^{1}$

${ }^{1}$ University Clinic of Urology, Skopje, Macedonia.

\section{Abstract}

Globally, trauma is the sixth leading cause of mortality, causing for $10 \%$ of all deaths and disability to many millions more. The kidney is the most frequently injured organ in the genito - urinary system. The renal traumatic injuries account for $10 \%$ of all abdominal trauma and up to $5 \%$ of all trauma cases.

Renal trauma is defined as a physical blunt injury or a penetrating wound of the renal parenchyma, renal vascular vessels or the urine evacuation structures induced by a physical extrinsic agent. Most common causes of renal trauma includes traffic incidents, falls, impacts during contact sport activities (blunt injuries) or knife and gunshot wounds (penetrating injuries).

The first step of clinical management of suspect renal trauma consists of evaluation including patient history collection and physical examination. The most essential aspect in the management of renal trauma involve patient's hemodynamic stability. For that purpose, it is important that the vital signs should be permanently monitored.

The commonly used Injury Severity Scale system for the kidney trauma is an anatomic-based description, developed by the American Association for the Surgery of Trauma (AAST). According to this system, the renal injuries are classified into 5 grades.

Treatment options are selected based on the advanced trauma life support (ATLS) guidelines, the development of strategies for controlling the hemorrhage by massive transfusions, and the use of damage control surgery procedures.
52. Injuries of Renal Artery and Vein in Abdominal Trauma

Arian HODO$^{1}$, Astrit MUSTAFA ${ }^{1}$, Bedri MIHAJ $^{1}$, Arben ZENELAJ ${ }^{1}$

${ }^{1}$ Urology Service, University Hospital of Trauma, Tirana, Albania

\begin{abstract}
Pedicle renal vascular trauma are mostly rare. In trauma associated with laceration of artery or vein mortality is high. Most cases of blunt trauma are caused by automobilistic accidents and rarely from penetrating trauma.
\end{abstract}

Objective: Presentation of our 8 year experience.

Material and methods: In the study are encluded cases of automobilistic and penetrated traumas of renal pedicula between 2007 to year 2015. In total 9 cases from which 5 cases resulted trauma of renal arteria associated with masiv or total thrombus of renal artery without laceration and not associated with masive retroperitoneal hematoma or multiorgan trauma. Concerning the late diagnoses these patients are treated conservatively, monitoring vital signs and urine output. Examination is done under emergency conditiones, Abdominal CT scan with contrast solution and a week after with renal schintigraphy. In DMSA renal schintigraphy resulted masive or total avascularisation. The patients are treated with antibiotics to prevent retroperitoneal abcess and in only one case we needed antihipertensive treatment. In three of the cases we performed nephrectomy because of masive retroperitonela hematoma with vitale failure. One of the cases was penetrating trauma of renal vein and vena casa $(4 \mathrm{~cm})$. Patient was diagnosed in Operating Room, it was a multiple organ trauma encluded pulmonary and hepar injury. In this patient we used part of renal vein flaping vena cava and anastomosis renal vein by transposition of kidney up to the healthy vena cava parts.

Conclusion: Because of the fact that patients with thrombus of renal artery where diagnosed relatively late, surgical 
intervention was not an option. As result, the patiens were followed up conservativly. In postoperatory period no complicationes were noticed.

Key words: trauma, vascular pedicle, hematoma, thrombus, conservative.

\section{Bladder Cancer Initial Approach in Emergency}

Gjergji SEMINI ${ }^{1}$, Grigorios DALIGAROS ${ }^{2}$, Kostandinos ZOTOS ${ }^{3}$, Athanasios LAZARIDIS ${ }^{4}$, Fotios DIMITRIADIS ${ }^{5}$

${ }^{1}$ Urologist, Amerikan Hospital Tirane, Albania

${ }^{2}$ Urologist, Ioannina, Greece

${ }^{3}$ Urologist, Kozani, Greece

${ }^{4}$ Urologist, Arta, Greece

${ }^{5}$ Urologist, Aristotle University, Thessaloniki, Greece

\section{Abstract}

The urinary bladder cancer (BC) is the eleventh most common cancer in both genders worldwide. About 2,4\% of total population will be diagnosed with bladder cancer in their lifetime. Men are affected 3 to 4 times more frequently than women, having also a double mortality rate.

In advanced countries about $90 \%$ of bladder neoplasms are urothelial carcinomas, occurring at the urothelial cells of the bladder wall. These lesions can also affect other areas of the urinary tract, such as the renal pelvis, ureters and urethra.

The habit of tobacco smoking, with the presence of polyaromatic hydrocarbons carcinogens in tobacco products, is the most important cause of bladder cancer. Other causative factors are: advanced age, family history, occupational exposure to types of organic chemicals (found in textile dyes, rubber, petroleum products, metals, processed leather and paints), arsenic exposure, previous chemotherapy and pelvic radiotherapy, chronic bladder inflammation/infections.

Bladder cancer signs/symptoms may include painless gross or microscopic hematuria (the most common) and irritative bladder symptoms \{more often in patients with carcinoma in situ (CIS) $\}$. Rare findings are pelvic/bone pain, lower extremity edema, flank pain and palpable mass. The diagnosis depends mainly on cystoscopy examination.

Initial treatment for most $\mathrm{BCs}$ involves transurethral resection (TUR) in order to remove all suspected lesions and obtain material for histological examination.

This article aims to discuss the diagnostic approach and the treatment modalities of bladder cancer.

Keywords: bladder cancer, diagnosis, treatment

\section{12 Years of Experience with Buccal Urethroplasty for Traumatic Ruptures of Urethra. A Review of 95 Cases. Results and Outcomes.}

Gezim GALIQI ${ }^{1}$, Shpetim YMERI ${ }^{1}$, Albert PESHA ${ }^{2}$, A. $\mathrm{KONI}^{3}$, Shkelqim FERKO $\mathrm{F}^{4}$, Bilbil HOXHA $\mathrm{H}^{3}$, Anton $\mathrm{NDOJ}^{4}, I$. VISHI ${ }^{5}$, D FERIZI ${ }^{5}$

${ }^{1}$ Regional Hospital of Shkoder, Albania.

${ }^{2}$ Regional Hospital of Fier, Albania

${ }^{3}$ Amerikan Hospital of Tirana, Albania.

${ }^{4}$ Regional Hospital of Durres, Albania

${ }^{5}$ Regional Hospital of Ferizaj, Kosovo

\begin{abstract}
Background: Posttraumatic reconstruction of a urethral stricture poses a difficult surgical problem. The use of buccal mucosa graft only urethroplasty represents the most widespread method of bulbar urethral stricture repair. The graft may be placed on the ventral or dorsal urethral surface according to surgeon experience and preference.
\end{abstract}

Aim: To represent our experience with Buccal Mucosa Urethroplasty (BMU) for substitution of all segments of urethra after urethral trauma.

Materials and Methods: We repaired 95 urethral strictures with BMU from 2006 2018. From them 42 were with traumatic origin. One stage dorsal or ventral urethoplasties were: Recurrence rate, complications were analysed retrospectively. Mean follow up was minimally 2 years. From one stage BMU in 28 cases was used ventral graft for bulbous 
urethra. In the rest of traumatic origin especially after obliterated strictures after pelvic fractures we used dorsal graft. Patient were evaluated in follow up with flusometry and when it was less then $10 \mathrm{ml} / \mathrm{sec}$ the Qmax with flexible cystoscopy.

Results: Median age of the patient was 49, mean length of the graf was 4.2. The success rate was $86 \%$. In patient with recurrence we did internal urethrotomies which resulted conclusive. Only in 5 patient we did again grafting with BMU.

Conclusion: One stage BMU provides an excellent result for strictures involving any segment of anterior and posterior urethra $(1,2)$. Even in the case of recurrence most of the patient are resolved with internal urethrotomies leaving redo BMU only for $5.2 \%$ of cases.

Keywords: Buccal urethroplasty, trauma, urethra, rupture, results

\section{The Use of S-Curved Coaxial Dilators for Urethral Dilatation. Experience of a Tertiary Department.}

Gjergji SEMINI ${ }^{3}$, Panagiotis KALLIDONIS ${ }^{1}$, Constantinos ADAMOU ${ }^{1}$, Adamantia KOUTAVA $^{1}$, Panteleimon NTASIOTIS ${ }^{1}$, Dimitrios KOTSIRIS $^{1}$, Evangelos LIATSIKOS ${ }^{1.2}$

${ }^{1}$ Department of Urology, University Hospital of Patras, Patras, Greece

${ }^{2}$ Department of Urology, Medical University of Vienna, Vienna, Austria

${ }^{3}$ Department of Urology, American Hospital, Tirana, Albania.

\section{Abstract}

Background: Urethral strictures can be treated by urethral dilation, optical internal urethrotomy or open surgical reconstruction (urethroplasty). The S-Curved Coaxial Dilator (SCCD) was designed to facilitate the passage of the dilator through the stricture and the urethra because it imitates the curved anatomy of the male urethra.

Aim: This study presents our experience with SCCD.

Materials and Methods: We used this kind of dilation in 310 patients. The technique included the insertion of a hydrophilic floppy tipped guidewire through the urethra directly into the bladder under fluoroscopic control. The SCCDs were then inserted over the guidewire. Dilators of gradually increased size from $8 \mathrm{~F}$ to $20 \mathrm{~F}$ were used. The follow-up of the patients includes uroflowmetry and measurement of post void residual (PVR) at 4 weeks, 6 months or in the case of a recurrence of symptoms.

Results: The age of the patients were 69.08 \pm 15.77 years. The causes of urethral stricture were iatrogenic $(\mathrm{n}=114 ; 36.8 \%)$, traumatic $(\mathrm{n}=35 ; 11.3 \%)$, infectious $(\mathrm{n}=$ $22 ; 7.1 \%)$, and of unknown origin $(n=139$; $44.8 \%$ ). The stricture length was $1.62 \pm$ $0.85 \mathrm{~cm}$. The mean number of dilations needed per case was 2 (range $1-15$ ) and the time between the dilations was $212.19 \pm$ 253.9 days. We had 7 failures.

Conclusion: We propose the S-Shaped Coaxial Dilators for urethral dilation as a safe and effective technique because of their similarity to the shape of the male urethra and because of their hydrophilic coating.

Keywords: SCCD, urethral strictures, dilation

\section{Sachse Laser in PostTrauma Urethral Stricture: A Valid Alternative before Urethroplasty.}

\author{
Astrit MUSTAFA ${ }^{1.3}$, Evisa ZHAPA ${ }^{1}$, Francesco \\ Saverio GROSSI ${ }^{1,2}$ \\ ${ }^{1}$ Urology Department, Italo-Albanian, "Salus" \\ Hospital, Tirana, ALBANIA \\ ${ }^{2}$ Complex Structure of Urology, Hospital SS \\ Annunziata of Taranto. ITALY \\ ${ }^{3}$ University Hospital of Trauma Tirana, ALBANIA
}

\begin{abstract}
Introduction: The most commonly used treatment modality for urethral strictures is the direct visual internal urethrotomy method according Sachse cold knife, but with low long-term success rate. Alternative method after failure is urethroplasty with preputial flap or Buccal mucosa. The aim of this presentation is to report the efficacy of the Internal Urethrotomy with Holmium Laser (Sachse laser).
\end{abstract}


Material and methods: We report three cases of urethral stenosis after trauma which treated with Internal Urethrotomy with Holmium Laser (Sachse laser) after failure of multiple treatments with Sachse cold knife. We evaluated and compared the uroflowmetry parameters before and after Sachse laser.

Results: The average of uroflowmetry parameters before incision were: Q $\max 4.5$ $\mathrm{ml} / \mathrm{sec}$, Q med $3 \mathrm{ml} / \mathrm{sec}$. Voided Volume $323 \mathrm{cc}$, while after incision with Sachse laser were: Q $\max 30 \mathrm{ml} / \mathrm{sec}, \mathrm{Q}$ med 12 $\mathrm{ml} / \mathrm{sec}$. Voided Volume $363 \mathrm{cc}$. No patients relapsed during follow-up time from 3 to 24 months.

Conclusion: Even though these are only a few cases, we think that Sachse laser is a valid alternative after unsuccessful Sachse cold knife and before. Urethroplasty, even in patients with urethral stricture after trauma.

Keywords: Treatment modality, urethral strictures, alternative method

\section{Treatment of Nocturia in $\mathrm{BPH}$ Patients with Tamsulosine 0,4 mg.}

Rezart XHANI ${ }^{1}$, Mustafa XHANI ${ }^{1}$, Eugen TATA ${ }^{1}$, Herion DREDHA ${ }^{1}$

${ }^{1}$ Urology Department, University Hospital Center

"Mother Teresa”, Tirana, Albania

\begin{abstract}
The use of Tamsulosine is safe and effective in men affected by BPH who present with nocturia symptoms. The study was done during 15 weeks and included 100 patients aged 55 years or older with 3 or more nocturia episodes during the night based on voiding diary. The IPSS was 8 or more (moderate symptoms) and patients were treated with Tamsulosine $0,4 \mathrm{mg}$. The number of nocturia episodes was reduced by 1,16 and the IPSS was clearly ameliorated after 15 weeks of treatment. The most common adverse effect reported was retrograde ejaculation ( $8 \%$ of patients). Keywords: nocturia, BPH, tamsulosine, treatment
\end{abstract}

\section{SHOCK - What, When, and How to Treat...}

John E. FRANCIS ${ }^{1}$

${ }^{1}$ Lafayette Surgical Clinic, Chief of Surgery,

Director of Trauma, Lafayette, Indiana, USA

\begin{abstract}
Shock is poorly understood term which encompasses a wide range of experiences. Hemorrhagic shock in particular has its own particular challenges with regards to treatment and mitigating effects of therapy. This discussion will focus on the current guidelines and recommendations for identifying, treating, and decreasing the risks associated with treatment of patient's in shock.

Shock is a word we use to describe inadequate tissue perfusion by oxygenated blood. In hemorrhagic shock there is a concomitant loss of fluid to carry oxygen and ability to remove cell products. This double-effect compounds the destructive aspect of decreased perfusion alone. We will discuss methods and techniques to reverse these effects as well as prevent ongoing damage from the initial insult as well as the effect of the treatment.
\end{abstract}

\section{Trauma and Coagulopathy}

Blerim ARAPI ${ }^{1}$, Gentian HUTI ${ }^{1}$, Asead ABDYLI ${ }^{2}$, Rinard KORTOCI ${ }^{1}$

${ }^{1}$ Anesthesiology and Intensive Care Service,

${ }^{2}$ American Hospital Tirana, ALBANIA

\section{Abstract \\ Introduction; Hemorrhagic shock from blood loss is a major cause of mortality in trauma patients. Bleeding is due to an intrinsic dysregulation of the blood coagulation known as trauma-induced coagulopathy (TIC). TIC is faced in the presence of both hypoperfusion from blood loss and tissue injury.}

Materia and Methods; We review the literature, in order to highlight the modern concepts and mechanisms contributing to 
TIC. Understanding these mechanisms can improve treatments strategies, morbidity, and mortality of severe traumatized patients.

Results; The mechanisms of coagulopathy in trauma patients have been explained by close examination of the biochemical and cellular changes due to tissue injury and hemorrhagic shock. Mechanisms contributing to TIC include anticoagulation, consumption, platelet dysfunction, and hyperfibrinolysis.

Conclusions; The anesthesiologist and intensivist often are faced with traumatized patients. The surgical team must be prepared to promptly treat trauma induced coagulopathy, and good knowledge about this complication is the first step.

Keyword: coagulopathy in trauma, blood coagulation, hypoperfusion, platelet dysfunction

\section{Cardiac Arrest in Anesthesia}

\section{Pirro PRIFTI ${ }^{1}$}

${ }^{1}$ Head of Department of Technical Medical Sciences, Faculty of Professional Studies

“Aleksander Moisiu”, University, Durres, Albania.

\section{Abstract}

Aim of this study is fast Recognition, Management of situation of Cardiac Arrest during Anesthesia and treatment of complications.

Objectives are: Analysis of Causes that trigger Cardiac Arrest, analysis of risk factors and prevention of Cardiac Arrest in Anesthesia.

Is known that Cardiac Arrest occur at 0.5/ 10.000 cases of Anesthetized Patients

Between other Causes, Human Mistake is one of cause of Cardiac Arrests.

Cardiac Arrest can be defined as a physiopathologic situation of acute Cardiac failure, when the Heart stop and cannot supply with blood -vital organs, mainly the Brain.

Causes of Cardiac arrest are multiple and more important are: Cardiac arrest with Reflector origins, direct cardiac trauma, overdose of anesthetic drugs, Ventricular
Fibrillation, acute coronary failure, acute diminished venous reflux, cardiac arrest from metabolic disorders, massive blood transfusions, anaphylactic reactions and cardiac arrest from special surgical procedures as prosthesis with implanting cement.

Symptoms during Anesthetized patients are poor, but Anesthesiologist must be very careful. Symptoms are, hypotension, cyanosis, dysrhythmia, hypoxia, hypercarbia, surgeon see ‘black blood` and asystole.

Mydriasis occur 45 second after Cardiac Arrest and is in fact a late symptom of Cardiac Arrest during Anesthesia

Cardiac Arrest con cause within 2-4 min irreversible changes that can cause death or permanent grave neurologic and psychiatric inabilities.

Treatment must be emergent within 20-40 sec after Anesthetist has diagnosed Cardiac Arrest and must include Interruption of surgical Operation, change of position of patient in surgical bed, interruption of Narcotic agents, giving of Oxygen 100\%, careful cardiac monitoring, and starting of CPR.

Therapeutic Principles are: Phase of CPR, Phase of Patient Ventilation and careful Monitoring, Faze of Diagnostication and Intensive Care Therapy.

Key Words. CPR, Anesthesia, Cardiac Arrest, Monitoring.

\section{The Role of Anesthesiologist in Burned Patients, Our Experience}

Nehat BAFTIU ${ }^{1}$, R. BAFTIU ${ }^{1}$

${ }^{I}$ Anesthesia and Intensive Care Service, University Clinical Center of Kosovo, Pristine, Kosovo.

\begin{abstract}
Introduction; Burns are epidermal tissue lesions that develop as a result of the pathological activity of the thermal agent, electric current, lightning, chemical substances or radioactive rays on the surface of the body. Burns are the third cause of accidental deaths. The treatment of
\end{abstract}


burned patients requires in many cases the involvement of anesthesiologist, where in 4 to $22 \%$ of cases these patients are treated in the Intensive Care Unit (ICU).

The purpose of this presentation is to highlight the role of the Anesthesiologist in Burning Patients, Therapy given to the Intensive Care Unit, and increase the survival rate in these cases when the treatment becomes multidisciplinary.

\section{Material and Methods}

The data from patient reports in MIQ (ICU) protocols were collected for a period of 2 years (January 2016-January 2018), from which data on the treatment of these patients were obtained, the length of stay in MIQ and the success rate in the survival of these patients. Comparisons were also made with the results of the same cases published by foreign authors.

Results; It is clear that the success of the treatment of burned patients requires teamwork and especially the involvement of anesthesiologist - reanimator. Parkland's formula for losing weight compensation was the basis of treatment for these patients, both adults and children. Analgesia was also included in the baseline treatment group. Prophylaxis from infection, Tetanus and ulcer stresses will be presented in this presentation. Given that about $47 \%$ of burns have burns in the face, burning patients' intubation remains the key to survival.

Conclusion; Anesthetist doctor has a key role in treating burnt patients. Knowing fluid loss compensation formulas, MIQ experience, timely intubation, and prophylaxis for infections increase the survival potential of these patients.

Keywords; Burning, Anesthesia, Intensive Treatment, Analgesia, Loss of fluid

\section{Epidural Analgesia in Patients Undergoing Open Abdominal Surgery, Our Experience}

Nehat BAFTIU ${ }^{1}$, R. BAFTIU ${ }^{1}$

${ }^{1}$ Department of Anesthesia \& Critical Care, University Clinical Center of Kosovo-Pristina, Kosovo

\begin{abstract}
Background; Epidural analgesia has been the reference standard for the provision of post-operative pain relief in patients recovering from major abdominal operations. However, in different studies, a failure rate of $20-32 \%$ has been reported.

The aim of the study was to analyse the success rates of epidural analgesia and the outcome in patients who underwent open major abdominal surgery.
\end{abstract}

Material and Methods; We collected data from a prospectively database of 50 patients who underwent open abdominal surgery during a period of 2 years (September 2017 to September 2017). Anaesthetic consultants with expertise in anaesthesia for open abdominal surgery performed the epidural catheter placement. A dedicated pain team assessed the post-operative pain scores on moving or coughing using the Verbal Descriptor Scale. The outcome was measured in terms of epidural success rates, pain scores, post-operative chest infection and length of hospital stay.

Results: The study group included 33 males and 17 females, most of them undergoing liver surgery. The indication for resection was liver secondaries (70\%), primary tumours (20\%) and benign disease (10\%). While major ( $\geq 3$ segments) and minor resections ( $\leq 2$ segments) were performed in $44 \%$ and $48 \%$ respectively, $8 \%$ of patients were inoperable. Epidural analgesia was successful in 46 patients $(92 \%)$. Bacterial colonisation of epidural tip was noticed in one patient. However, no neurological complications were encountered. Five patients (10\%) had radiologically confirmed chest infection. Three patients (6\%) developed wound infection. One patient died due to liver failure following extended right hepatectomy and cholecystectomy for gall bladder cancer. The median length of stay was 6 days (3-25 days).

Conclusions: Our experience shows that epidural analgesia is safe and effective in providing adequate pain relief following major open abdominal surgery. 
Keywords: Epiural analgesia; open abdominal surgery; acute pain; prevention

\section{Updating Septic Cascade and Microbial Resistance}

Mihal KËRÇI ${ }^{1}$, Selim HORESHKA ${ }^{1}$, Elona NAQELLARI ${ }^{1}$

${ }^{1}$ University of Medicine, Tirana, ALBANIA. Continental Hospital, Tirana, ALBANIA

Abstract
Sepsis represents the systemic inflammatory response and the presence of the infection. The Consensus Conference of 1992 introduced the term SIRS, which includes patients with a minimum of two of the following details:

Sepsis is a life-threatening situation that occurs when the body's response to the infection causes damage to tissues and organs. In the first two consensus, Sepsis 1 and 2 for gravity interpretation used SOFA score, with a scale from 0 to 4 points, while the current concepts for patients suspected to be septic, who have a chance to stay tall in TI or die in the hospital can be identified with the Rapid Sequential Organ Failure Assessment (qSOFA), and the score drops up to 3 points, referring to mental status alteration, PAS $\leq 100 \mathrm{~mm} \mathrm{Hg}$, or FR $\geq 22$ / min.

Temperature $>38^{\circ} \mathrm{C}$ or $\left\langle 36^{\circ} \mathrm{C}\right.$; FC $\rangle 90$ in min; FR> 20 / min or $\mathrm{PaCO} 2<32 \mathrm{mmHg}$; leukocytes> $12000 \mathrm{~mm} 3$ or $>4000 \mathrm{~mm} 3$ and $10 \%$ or more of imatine forms.

Sepsis (SIRS + Infection), Serious Sepsis (Sepsis + Organ Traumatic), and Sepsis Coma (Serious Cardiac Vascular Sepsis which does not respond to the use of lice, but the need for vasopressors is felt), but the same concept was that of consensus for Sepsis 2 in 2001, while Sepsis 3 recommends the clinical use of the above elements but being limited to the Sepsis term and the Septic Sniper.

Conclusion; New advances in sepsis and septic shock have shown improved survival in these critical patients. Sepsis: "life threatening from organ dysfunction caused by a disordered response of the recipient to the infection".

\section{Surgical Treatment of Combined Acetabulum and Pelvic Ring Fractures}

\author{
Jordan SAVESKI $^{1}$, Neda TRAJKOVSKA ${ }^{1}$, Ilir \\ $\mathrm{HASANI}^{2}$ \\ ${ }^{1}$ Clinical Hospital Acibadem Sistina, Skopje, \\ Macedonia \\ ${ }^{2}$ University Clinic of Traumatology, Medical \\ Faculty, Skopje, Macedonia
}

\begin{abstract}
Introduction: Combined fracture of acetabulum and pelvic ring fracture (CAPRF) usually are results of high energy trauma and occur in polytraumatized patients. Hemodynamically unstable patients in this injuries present unique treatment dilemmas and special challenge for the treating surgeon.

The purpose of this study is to present our experience with surgical treatment of CAPRF injuries.
\end{abstract}

Material and methods: From 1995 to 2014 year, 68 patients $(15,1 \%$ of 446 pt with pelvic ring fractures) with CARPF were surgically treated. Of all of them $72 \%$ were polytraumatized, $\quad 32 \% \quad$ were hemodynamically unstable with average ISS of 30.6. The most common cause of injurie was MVA - 69\%, fall from height $20 \%$ and other $-11 \%$. There were 42 males and 26 female pt. Pelvic ring fracture (PRF) were classified by Young-Burgees: APC in $50 \%$ (34pt), LC in $45.6 \%$ (31 pt), VS in $2.9 \%$ (2 pt) and combined in 1,5\% (1 pt). Acetabulum fracture were classified by Letournel - Judet: TR - 23 pt, TR+PW - 2 pat, ABC - 16 pt, ACPH - 12 pt, TT- 5 pt, $\mathrm{AC}-5 \mathrm{pt}, \mathrm{AC}+\mathrm{AW}-2 \mathrm{pt}, \mathrm{PW}-2 \mathrm{pt}$ and $\mathrm{PC}$ $1 \mathrm{pt}$.

Results: Assessment of the X-Rayresults were done according Matta: excellent - 44 (64\%), good - 10 (14\%), fair - $8(12 \%)$ and poor $6(10 \%)$. Final functional results were done according Poste 1D'Aubigne score system: excellent - 32 (46\%), good - 22 (32\%), fair - 7 (11\%) and poor - 7 (11\%). 
Conclusion: CAPRF are results of high energy trauma and occur in polytraumatized patient. Incidents of APC and LC of this injury is similar. Transverse and both column acetabulum fracture are the most common fracture patterns. The high incidence of associate injuries mandates multidisciplinary treatment approach. This injuries should be accessed separately and the overall treatment strategy should integrate the independent assessment of the pelvic ring and acetabulum fracture. The treating surgeon should strive to achieve the anatomic reduction of both pelvic ring and acetabulum fractures for good functional outcome.

\section{Management of Severe Pelvic Trauma}

\section{Fausto CATENA ${ }^{1}$}

${ }^{1}$ Professor of general surgery, Chief Department of Emergency and General Surgery Parma University Hospital ITALY.

\section{Abstract}

Complex pelvic injuries are among the most dangerous and deadly trauma related lesions. Different classification systems exist, some are based on the mechanism of injury, some on anatomic patterns and some are focusing on the resulting instability requiring operative fixation. The optimal treatment strategy, however, should keep into consideration the hemodynamic status, the anatomic impairment of pelvic ring function and the associated injuries. The management of pelvic trauma patients aims definitively to restore the homeostasis and the normal physiopathology associated to the mechanical stability of the pelvic ring. Thus, the management of pelvic trauma must be multidisciplinary and should be ultimately based on the physiology of the patient and the anatomy of the injury. Our aim is to present the World Society of Emergency Surgery (WSES) classification of pelvic trauma and the management Guidelines.
66. Anterior Pelvic Approach.

\author{
Vilmos VÉCSEI ${ }^{1}$ \\ ${ }^{I}$ Dept. for Orthopedics and Trauma Surgery, \\ Medical University Vienna, Austria.
}

Abstract
Introduction; The anterior ilioinquinal approach introduce by Emil Letournel and Robert Judet offering the access to the anterior column of the acetabulum and to the ala ossis ilei, so fractured. is very well known. The view to the bony structures through the 2 windows medial (lacuna vasorum) and lateral (lacuna musculorum with the femoral nerv) visualizes the pubic bone and the anterior column of the acetabulum. The linea terminalis and the quadrilateral surface are more palpable than visible. The preparation for this approach should not be underestimated. The N. cutaneus femoris lateralis, the $\mathrm{N}$. femoralis, the femoral vessels and the corona mortis require a skilled surgical procedure.

The Stoppa approach opens good access to the anterior column, the the linea terminalis, the alla ossis ilei and is suitable to deal with anterior pathologies. Posterior column combined with posterior wall fx., transverse fx. combined with posterior wall fx., transverse fx. and posterior wall fracture require a posterior approach, possibly combined approaches.

The Stoppa approach offers clear advantages if you pay meticulous attention to the protection of vulnerable and endangered anatomical structures, i.e. femoral vessels, corona mortis, $n$. obturatorius, the urinary bladder.

The incision of the skin performed in transverse (or longitudinal) direction, opening of the abdominal wall along of the linea alba locking for the way preperitoneally after the disinsertion of the rectus muscle on his insertion site in the direction of the linea terminalis. Ligation of the corona mortis, Incision of the pectineus fascie and lift up of the iliopsoas muscle. It is recommended to use special radiolucent Hohmann hooks with the possibility to fix them to the adjacent bony structures with $\mathrm{K}$ wires. 
Fracture reduction and fixation are the last steps.

Training in cadaver labs is recommended.

\section{Advances and Challenges in Anterior Approaches on the Treatment of Acetabular and Pelvic Fractures}

Ilir HASANI ${ }^{1}$

${ }^{1}$ General Surgeon - Traumatologist, Traumatology Orthopaedic Surgery, Anesthesy, Intensive Care and Emergency Centre, Skopje, MACEDONIA.

\begin{abstract}
Anterior approaches have made revolution on the treatment of acetabular fractures in the middle of the last century with the scientific work of Letournel.

Anterior Intra-Pelvic Approach has been promoted at the end of the last century, as new and alternative approach on treating the fractures of the acetabulum, involving anterior structures. Since its promotion from Hilvensaro and Cole, it has gained a worldwide reputation of a save and successful alternative of ilio-inguinal approach of Letournel, which was a leader approach after it was Letorunel has introduced it in traumatology experience.

In the Republic of Macedonia, ilio-inguinal approach has been routinely used in the Traumatology Clinic in the last decades with very good results. In contrary to its reputation worldwide, the Anterior Intrapelvic Approach has not been reported in the Medical literature in the Republic of Macedonia.

We present our experiences with the fist intrapelvic approaches and infrapectineal placement of the plate, challenges, future development and expectations following trends refered in the literature.
\end{abstract}

Key Words: Anterior approach, acetabular fracture, anterior intrapelvic approach, modified Stoppa approach

\section{Unstable Pelvic Ring Injuries - Classification and Treatment}

Sasa MILENKOVIC ${ }^{1}$

${ }^{1}$ University of Nis, Medical faculty, Orthopaedic \& Traumatology Clinic Nis, SERBIA

\begin{abstract}
Introduction: In general, pelvic ring injuries can be caused by low-energy trauma in older patients with osteoporosis and by high-energy trauma in younger patients. Pelvic ring injuries can be isolated or accompanied by other injuries of the locomotory apparatus, injuries of small pelvic organs, blood vessels and nerves as part of polytrauma. In accordance with this, patients can be hemodynamically stable or hemodynamically.unstable, their lives may be threatened. Controlling and stopping bleeding in hemodynamically unstable patients is achieved by the pelvic clamp, external skeletal fixation, pelvic tamponade or embolization. Unstable injuries to the pelvic ring require stabilisation of the anterior or anterior \& posterior pelvic ring complex.
\end{abstract}

Methods In this retrospective study patients with unstable pelvic ring injuries were followed between 2013. and 2017. Hemodynamically unstable patients with unstable pelvic ring injuries were treated by external fixation using Malkovich's external fixator. Final pelvic stabilization was performed by pelvic ring osteosynthesis. There are several classification of the pelvic ring injiries: anatomical (Letournel), Georg Pennal, Joung\& Burgoss, Marvin Tile and AO classifitaion. We used Tile's classification, the final outcomes had been scored using Majeed scoring system.

Results We presented 31 patients with a pelvic ring injury (Type B-14, Type C-17). Primary fixation was performed by external fixation or skeletal traction in hemodynamically unstable patients. Final stabilization consisted of external fixation or anterior and posterior pelvic ring complex osteosynthesis. There were 3 $(9,67 \%)$ cases of DVT, $1(3,22 \%)$ infection, $2(6,45 \%)$ cases with pelvic nonunion, 1 
$(3,22 \%)$ fixation failure. Final functional outcome was excellent in $14(45,16 \%)$, good in $13(41,93 \%)$, fair in $3(9,67 \%)$ and poor in $1(3,22 \%)$ case.

Conclusion Pelvic ring injuries are severe and its treatment requires a team work. External fixation has been approved as a good primary fixation method in ploitraumatized and hemodynamically unstable patients. Final surgical treatment requires stabilization of the anterior pelvic complex fixation, in type B injuries, and both anterior and posterior pelvic ring complex, in type $\mathrm{C}$ injuries.

\section{Management of Esophageal Perforations}

\section{Carlos MESQUITA ${ }^{1}$}

${ }^{1}$ Senior Consultant of General \& Emergency Surgery at Coimbra Central and University Hospital, COIMBRA, PORTUGAL

\section{Abstract \\ The esophagus is more vulnerable than the rest of the alimentary tract, due to the lack of a serosal layer, and perforation may be due to several mechanisms. Iatrogeny, due to a number of procedures as endoscopy and dilatation of strictures, accounts for almost $75 \%$ of esophageal perforations. Spontaneous rupture of the esophagus, a dangerous entity known as Boerhaave's syndrome, accounts for about $15 \%$. \\ Perforating trauma, foreign bodies and toxic ingestions make up the bulk of the remaining causes, around $10 \%$. Because the esophagus is surrounded by loose stromal connective tissue, the infectious and inflammatory response can disseminate easily to nearby vital organs, thereby increasing the likelihood of serious sequelae, making the esophageal perforations, in general, potentially devastating conditions. Although rapid diagnosis and therapy offer the best chance of survival, delays are common for a multitude of reasons, resulting in substantial morbidity and mortality.}

\section{Management of Solid Injured Organs in Blunt Abdominal Trauma: NOM vs OM}

\author{
Mauro ZAGO \\ ${ }^{I}$ General Surgery Dept, Minimally Invasive Surgery \\ Unit, Head Policlinico San Pietro - Bergamo - \\ ITALY*
}

\section{Abstract.}

During the past decades, nonoperative management of liver, spleen and kidney injuries has revolutioned the therapeutic options. The typical patient who underwent non operative management has to be haemodynamically stable or rapidly stabilized following initial resuscitation. Surgery continues to be considered the gold standard for haemodynamically unstable patients. Even if criteria influencing the risk for failire of NOM are well established, each patient should be managed on individual basis. Debatable points concern the role of angioembolization in both low and high AAST grade injuries, indications to NOM with associated injuries, the role of interventional radiology as rescue tool after Damage Control Surgery, the ideal followup algorithm. Nonoperative management of blunt solid organ injuries can be applied only following strict institutional protocols and according to the Hospital and trauma network resources.

\section{Management of Liver Trauma}

Carlos MESQUITA ${ }^{1}$

${ }^{1}$ Senior Consultant of General \& Emergency Surgery at Coimbra Central and University Hospital, COIMBRA, PORTUGAL

\section{Abstract}

The liver is one of the most frequently injured organs in abdominal trauma, due to its anterior location in the abdominal cavity and a fragile capsule and parenchyma. Any surgeon who has been confronted with a patient with a major hemorrhage due to a liver injury will witness the sense of weakness brought by this experience. To improve outcomes in patients with severe liver injuries, the surgeon needs a deep 
understanding of the pathophysiology, a knowledge of the anatomy, including the venous and arterial supply, a full armamentarium of surgical techniques, not always conventional, and a trained team, in an operative and resuscitation environment with the necessary equipment. Proper decision making is essential to a successful outcome. There is a paradigm shift in the management of liver trauma, due to better monitoring and diagnosis. In hemodynamically stable patients, nonoperative management of both low- and high-grade injuries can be successful. In hemodynamically unstable patients, direct suture ligations of bleeding vessels, vascular isolation with repair of venous injuries in the context of a damage control philosophy improved outcomes. Anatomical resections of the liver became rarely indicated. In general, the most appropriate technique is the simplest and quickest technique that can restore homeostasis. As soon as the patient becomes cold, coagulopathic and in irreversible shock, the battle is usually lost.

\section{Management of Blunt Abdominal Trauma in Children.}

Emir Q. HAXHIJA ${ }^{1}$

${ }^{1}$ Department of Pediatric and Adolescent Surgery, University Hospital Graz, Graz, Austria

\begin{abstract}
Introduction: Trauma is the leading cause of death in children. Abdominal trauma, most commonly blunt in nature, rarely requires emergent surgical intervention however at the same time it is the most common cause of unrecognized fatal injury in children. High energy accidents such as motor vehicle crashes and/or children hit by a car are the most frequent injury mechanisms. Spleen and liver as the two superficially lying solid organs are most commonly injured, followed by injuries to kidneys, pancreas and bowel. Nonoperative management for solid organ injuries can be employed to more than $90 \%$ of this patient population assumed close monitoring in
\end{abstract}

pediatric trauma centers is provided. Injuries to pancreas are rare and the indications for surgery more difficult to meet. Injuries to intestine with suspected bowel perforation are also less common but always require operative management. Intestinal injuries can be overseen on the initial Focused Assessment with Sonography for Trauma and/or Computed Tomography scan but will clinically present as severe peritonitis usually latest at 6-hrs post injury. Initial management in shock includes fluid boluses and transfusion with packed red blood cells in hypotensive patients after the second fluid bolus. Emergent laparotomy is indicated in patients with hemodynamic instability despite maximal resuscitative efforts. The diagnostic laparoscopy as initial surgical intervention in patients with abdominal trauma is increasingly applied to rule out a bowel, mesentery, or diaphragmatic injuries, especially in cases when emergent laparotomy is not needed. Laparoscopy has been shown to prevent unnecessary laparotomies and increase the postoperative quality of life.

\section{Hollow Viscus Injury due to Blunt Abdominal Trauma, a retrospective evaluation.}

Agron DOGJANI ${ }^{1}$, Engjellushe JONUZI ${ }^{1}$,

Shkëlzen OSMANAJ ${ }^{1}$, Kastriot HAXHIREXHA ${ }^{2}$

${ }^{1}$ Surgeon in University Hospital of Trauma, Tirana, ALBANIA

${ }^{2}$ Clinic Hospital of Tetovo, MACEDONIA

\section{Abstract}

BAT is one of the most common causes of morbidity in that all age groups. Early identification of traumatic hollow viscus injuries (THVI) has been and remains a challenge to all trauma surgeons, as early finding and evaluation of their, it present difficulties of different natures, even with technological advances is a frightening for all surgeons because of morbidity and mortality that it carries. With all the different varieties of damage mechanisms 
HVI of motor vehicle accidents (MVA) remain the leading cause ${ }^{1,2}$.

The first reports date for HVI we must go back to 1899 by Geille, who reported that HVI had $11 \%$ incidence in a group of patients who had undergone BAT. There are studies that said that TII recently ranked third overall in the standings intraabdominal injuries (IAI) in $\mathrm{BAT}^{3}$.

Objectives: Identification of the mechanism of injury, the incoming data, the anatomical distribution of lesions, diagnostic methods, management and gastrointestinal damage results from BAT.

Material and Methods: The study includes patients with BAT in a 2-years period, that were introduced in the UHT, who underwent evaluation, treatment and management of gastrointestinal tract injury. It is a retrospective study, patients were analyzed in relation to the mechanism, time of presentation, in HdSt, anatomic localization of lesions, diagnostic methods, the tactics of treatment, complications, morbidity and mortality.

Results: Are taken into consideration about $21(8 \%)$ patients out of 257 which is the total number of patients with BAT in the study which had damage to the HVI, they occupy $33 \%$ of cases treated with BAT street operators.

Age distribution is such 70 years old range 5-years average 32.8, while such is distribution by age group; is 14 to 60 years old $(6 \%)$ followed by more than 60 years old $(14.2 \%)$ and finally by $0-14$ years old (23.8\%).

Conclusion: Early recognition of THVI from BAT is difficult, it's very important is their dangerousness be recognized because of its great potential complications affecting infection that gives directly to their morbidity and mortality.

Key words; Blunt abdominal trauma, intestinal injury, colonic injury

\section{The Selective Ligature of the Hepatic Artery in a massive trans Thoracic Hemorrhage.}

Dorina SHTJEFNI ${ }^{1}$, Skender BUCI ${ }^{1}$

${ }^{1}$ Surgery Service, University Hospital of trauma, Tirana ALBANIA

\begin{abstract}
Introduction: The complex thoracoabdominal trauma is rare but life threating and very difficult to be managed. It is associated to a high morbidity and mortality. The goal of this study is to describe the diagnostic aspects and the management of the complications associated to this trauma.
\end{abstract}

Material and Methods: We will report the case of a hepatic pseudo aneurysm in a patient with the thoracic trauma and the rupture of the right diaphragm complicated with massive trans-thoracic hemorrhage.

Results: A 44 years old male, the patient was introduced to the ER after being hit by a heavy object. The CT evaluation revealed fractures of the 5-6-7-8-9-10-th ribs with hemopneumothorax and central hepatic contusion. The first treatment: drainage of the right pleural space broad spectrum parenteral, antibiotic therapy perfusions and follow up of the patient. The day after the pleural drain has been removed, we noticed biliar effusion coming out from the drainage hole. A new CT evaluation 7 days after the first one confirmed the presence of liquidienne multilocular collections in the right pleural space,the rupture of the right diaphragm and contusio of the 7-9 hepatic segments.10 days after trauma was performed the right thoracotomy and both organised hemothorax and biliar empyema was evacuated and the diaphragm was suturated.16 days after trauma the patient developed hemobili because of a fissuration of a pseudo aneurism in the central hepatic biliary way. The treatment for it was the administration of the kher drainage. After many episodes of hemorrhage associated to the hepatic pseudo aneurism and because we could not perform the hepatic artery embolization. The staff decided to perform the ligature of the right branch of the hepatic 
artery 4 weeks after trauma. The patient left hospital healthy 40 days after trauma.

Conclusions: The thoraco -abdominal complex trauma needs a multidisciplinar follow up. A very well-trained trauma staff and the disponibility of all medical equipments. Beside accurate management this trauma still has a high morbidity and mortality.

\section{Space-Occupying Cerebellar Infarction: Complications, Treatment, and Outcome}

Arsen SEFERI ${ }^{1}$, Artid LAME ${ }^{1}$, Ridvan ALIMEHMETI $^{1}$, Ejona LILAMANI ${ }^{1}$, Mirel GRADA $^{1}$, Arben RROJI ${ }^{1}$, Mentor PETRELA ${ }^{1}$ ${ }^{I}$ Service of Neurosurgery and Neuroradiology, University Hospital Center "Mother Theresa" Tirana, ALBANIA.

\begin{abstract}
Space-occupying edema is also a common complication in $17 \%-54 \%$ of patients with significant cerebellar infarction and may cause life-threatening conditions such as (1) obstructive hydrocephalus attributable to the mass effect in the posterior fossa resulting in blockage of the fourth ventricle, (2) direct compression of the mid- brain and pons, (3) upward herniation of the superior cerebellum through the tentorial incisura, and (4) down- ward herniation of the tonsils through the foramen magnum.

About $50 \%$ of patients who has evidence of mass effect from cerebellar edema showed subsequent clinical deterioration.

The first step of neurological manifestations of space-occupying cerebellar infarction is conservative treatment but a very meticulous clinical observations of cerebellar and sings of brain stem compression would determine the following surgical treatment. The role of repetitive radiological image is very important to choose the type of approach which consist; DVE, decompressive suboccipital craniotomy and removing the cerebellar necrotic issue.
\end{abstract}

We present our experience with four patients treated surgically for cerebellar infarction in posterior inferior cerebellar artery

\section{Epidemology of 1000 Cases Operated for Traumatic Injuries of Peripheral Nerves}

Ridvan ALIMEHMETI ${ }^{1}$, Gramoz BRACE ${ }^{1}$, Ermira PAJAJ $^{1}$, Alda KIKA ${ }^{2}$, Jetmira KERXHALLIU ${ }^{1}$, Norik BARDHI ${ }^{1}$, Myfit SARACI ${ }^{1}$

${ }^{I}$ Department of Neurosciences, University of Medicine, Neurosurgery Service, University Hospital Center "Mather Theresa" Tirana, Albania ${ }^{2}$ Department of Informatics, Faculty of Natural Sciences, University of Tirana. Albania.

\section{Abstract}

Introduction: Traumatic peripheral nervous system injuries benefit from surgery at specialized centers. The study presents the epidemiological data of the 1000 successive cases operated at the Neurosurgery Service, University Hospital Center "Mather Theresa" Tirana, Albania.

Material and Methods; Are Revised clinical files, operating registers and photos of cases operated for peripheral nervous system injuries.

A program for recording the main data of the operated cases has been built, where computerized data is precisely and in real time processed according to the categories. The data collected per 1,000 operated patients are included and processed to study epidemiologically.

Results; The characteristics that further influence the final outcome of the microscopic surgical repair of the peripheral nerves following traumatic lesions such as trauma type, level of injury, time from injury to final operation, lymphoid neoplasms, age were studied.

Conclusions; Neuro-surgical repair of traumatic damages of the peripheral nerves near the Neurosurgery Service, recognizes all categories of traumatic damages. Computerization for data processing and recording helps greatly in the precision of epidemiological data and constitutes the base that, with the help of data mining, also 
enables predicting the outcome of the operation in new cases.

Keywords: traumatic Injuries, peripheral nerve injurues, nerve transplant, microscopic surgical repair

\section{The Role of the Emergency Doctor in Pacients with Traumatic Injuries to the peripheral nerves}

Ridvan ALIMEHMETI ${ }^{1}$, Gramoz BRACE ${ }^{1}$, Ermira PAJAJ $^{1}$, Jetmira KERXHALLIU ${ }^{1}$, Norik

BARDHI $^{1}$, Mishel QIRINXHI ${ }^{1}$

Department of Neurosciences, University of Medicine, Neurosurgery Service, University Hospital Center "Mather Theresa" Tirana, Albania Abstract

Introduction; In the hospital and health care centers of the first aid for the involuntary involvement of the peripheral nerves, the assessment of the damaged structures and the degree of injuries is done. On this basis, the injured person is treated in these centers or referred to the most specialized centers for special competence to repair damaged peripheral nervous structures. Traumatic damages to the peripheral nerves benefit from correct surgery.

Material and Methods; Based on the experience of the last 25 years of the Neurosurgery Service, University Hospital Center "Mather Theresa" Tirana, Albania. in the contemporary treatment of traumatic damage of the peripheral nerves has studied the time spent by the injury and processing of the plage until the patient's arrival in our attention.

We have also studied the time relationship from damage to recapture at our Service, with the type of repair needed: end-to-end neurora, nervous transplant, neurolize. Results; From the studied cases it turns out that delayed cases from hospital and hospital centers for peripheral nervous system injuries to Neurosurgery Service, are delayed with the passing of time due to direct information to emergency physicians and specialist surgeons during the neurosurgery cycle.
This directly affects the best results at times treated as due to the shortest time in favor of muscular atrophy after denervation as well as for the type of treatment with less obstruction to nervous regeneration after repair (direct neurorafe, transplantation with short).

Conclusions: Treatment of peripheral nerves requires training in microscopic surgery of the surgeon who takes charge of the case, presence of microsurgical instruments and microscopes (or lupals), micro suture $(10 / 0,9 / 0,8 / 0)$. In the event of non-occurrence of one of these elements necessary for the repair of the peripheral nervous system damaged cases should be referred immediately after trauma in centers specialized for microscopic surgery and the competence to repair the peripheral nerve damage.

Keywords: peripheral nerves, traumatic damage, muscular atrophy, denervation...

\section{Balloon Kyphoplasty for Traumatic and Osteoporotic Compression Vertebral Fractures, our Experience.}

Artid LAME ${ }^{1}$, Gledion FEJZO ${ }^{1}$, Erion $\mathrm{SPAHO}^{2}$

${ }^{1}$ Department of Neurosciences, University of Medicine, Neurosurgery Service, University Hospital Center "Mather Theresa" Tirana, Albania

${ }^{2}$ Neurosurgery Service, University Hospital of

Trauma, Tirana, Albania

\section{Abstract \\ Introduction; We present a review of patients diagnosed and treated for osteoporotic thoracic-lumbar compression fractures.}

Materials and methods; There were three patients diagnosed and treated for osteoporotic thoracic-lumbar compression fractures during the year 2018 in a private hospital. The patients' clinical data, imaging studies and operative procedures were reviewed retrospectively.

Results; There were three female patients ranging from 34 to 63 years old, (mean age 51.6 years old) identified. All three patients were diagnosed previously for 
osteoporosis. Time from symptoms onset to diagnosis and treatment varies from one month to three years.

Discussion; Vertebral osteoporotic compression fractures are almost twice as common as other fractures typically linked to osteoporosis, such as broken hips and wrists. They are located more in the lumbar and thoracic region.

Conclusion; Treatment with balloon kyphoplasty remains the gold standard in the treatment of Vertebral osteoporotic compression fractures with early significant improvement of symptoms.

Keywords: osteoporotic fractures, compression fractures, balloon kyphoplasty

\section{The Repair Surgery of Peripheral Nerves from Gunshot Injuries}

Ridvan ALIMEHMETI ${ }^{1}$, Gramoz BRACE ${ }^{1}$, Ermira PAJAJ $^{1}$, Arba CECIA ${ }^{2}$, Thoma KALEFI ${ }^{1}$, Jetmira KERXHALLIU $^{1}$, Norik BARDHI ${ }^{1}$

${ }^{1}$ Department of Neurosciences, University of Medicine, Neurosurgery Service, University Hospital Center "Mather Theresa" Tirana, Albania ${ }^{2}$ George MassonUniversity, Faifax Virginia, USA.

\author{
Abstract \\ Introduction: Gunshot injuries to the \\ peripheral nerves has been commonly \\ encountered in recent years in the practice \\ of the Neurosurgery Service, University \\ Hospital Center "Mather Theresa" Tirana, \\ Albania. Repair of plexusor \\ peripheralnerveinjurues, represents the \\ difficultyassociated with the \\ damagemechanism, the coexistence of \\ causaligiavascularpreopreatore, bone or \\ tissueinjuries or otherinvolvedorgans. \\ Material and Methods; The \\ retrospectivestudy of the \\ casesoperatedisbased on clinicalrecords, \\ operator registers and photos of \\ casesoperated for \\ peripheralnervoussystemfrom \\ gunshotdamage. The \\ factorsthathavemostaffected the \\ postoperative progress of the \\ studycasessuchaspatientage, degree of
}

involvement of damagednerves, localization of injury, type of necessaryrepair, presence of preoperativecausalis, interest in vascularstructures or apparatuslocomotor.Therehasbeen a long follow-up in special cases over 10 years. Results; Peripheral and brachial nerve repairs in case of gunshot damage are a result of a good result if they are carried out in the near future by trauma, especially with regard to the treatment of causality that often accompanies these cases. The type of repair varies from aderenciolize, longitudina lepineurotoma, interferogularneurolize, neurorafi, nerve transplant. The degree of improvement depends mainly on the type of reparation needed and the distance from the damage to the effectormuscles.

Conclusions: In the perennial peripheral nervous system repair experience at the Neurosurgery Service, itturns out thathealingis more effectiveespecially in the treatment of pain and associatedmuscularatrophy.Tracking these years of ill will allow for regression blockage findings in distal anatomical tunnels of gunshot damage, which benefit from decompression even at a distance of several years from the time of the injuries. Keywords: Gunshot Injuries,peripheral nerve injurues, nervetransplant

\section{The Sensation is the Attribution of our Total Body Water}

Apostol VASO ${ }^{1}$

${ }^{1}$ University Hospital of Trauma, Tirana, ALBANIA

\begin{abstract}
The sensation results in an unperceivable event which happens in the sequence between two smallest units of time, because the attribution of the total body water (the ideal sphere) is to be sensitive even to the smallest particle of matter. Hence, the sensation is the attribution of the total body water, but this is the starting point or the first principle of our perception too. Since the ideal sphere feels the
\end{abstract}


nothingness, we can't operate with empiricism on it, hence the only way is to speak about it, with the logic or the Euclidean geometry.

\section{Rehabilitation of Patients with Cerebral Contusion}

Manushaqe SARAÇ ${ }^{1}$, Luljeta STANAJ ${ }^{1}$

${ }^{1}$ Rehabilitation and Physiotherapy Service, University Hospital of Trauma, Tirana, Albania

\begin{abstract}
Background: Head traumas are one of the most frequent causes of disability in adults. Cerebral injuries lead to motor, cognitive and behavioral deficits. Rehabilitation after Cerebral Contusions (CC) is an important part of the treatment strategy.
\end{abstract}

The aim this study was to evidence the importance of early rehabilitation of patients with $\mathrm{CC}$ which leads to prevention of complications and initiation of motor commands usage.

Material and Methods: This is a prospective study, including 167 patients, conducted in timeframe January 2017 to January 2018. The rehabilitation protocol has consisted of four phases: rehabilitation of patients in coma, prevention of complications, initiation of motor commands and autonomy or recovery phase. Ashworth scale is used for evaluation of spasticity on the first day (D1) of the month (M 1, 3, 6, 9, 12). The criteria for progression phase: 1 Surveillance of vital parameters (HR, RR, SBP, $\mathrm{SaO}_{2}$, Temperature), 2-MMT $3^{\text {rd }}$ degree (full ROM against gravity), 3Associated Injuries.

Results: 167 patients were rehabilitated with CC, 136 males (81.4\%) and 31 females $(18.5 \%)$, average age of $50 \pm 5$ years old. 132 patients (79.04\%) are fully rehabilitated within 6 months, 4 patients (2.39\%) remained with $2^{\text {nd }}$ degree of spasticity according to the Ashworth scale at the end of the $9^{\text {th }}$ month, 3 patients $(1.79 \%)$ remained with $1^{\text {st }}$ degree spasticity at the end of the $9^{\text {th }}$ month, 16 patients (9.58\%) (Non-reversible coma) remained with $4^{\text {th }}$ degree spasticity at the end of the $12^{\text {th }}$ month. 12 patients $(7.18 \%)$ died in the first month.

Conclusions: Early rehabilitation of patients with CC leads to prevention of complications and initiation of motor commands usage.

Keywords: Head Trauma, Disability, Ashworth scale, rehabilitation.

\section{Results of Pemberton Acetabuloplasty in Children with Developmental Dysplasia of the Hip (DDH).}

Gjergji ÇAUSHI ${ }^{1}$, Blerta HAMOLLI ${ }^{1}$, Arnel MICI ${ }^{1}$ ${ }^{1}$ Orthopaedic and Trauma Service, University Hospital of Trauma, Tirana, ALBANIA

\begin{abstract}
Aim: This study compared results following Pemberton acetabuloplasty in late presented children with development dysplasia of the hip (DDH). We assessed indications, contraindications, preoperative planning as well as details regarding Pemberton technique.

Materials and Methods: We studied 50 cases of children with developmental dysplasia of the hip presented in our department over 18 months old. Depending on the case they were treated with single stage open reduction and Pemberton acetabuloplasty or combined with adductor myotomy, skeletal traction or derotator osteotomy of the femur shaft.
\end{abstract}

Results: We present long term results from literature and also from our department. We present risks and complications of the surgical technique. We present indications of single stage open reduction and Pemberton acetabuloplasty or combination with adductor myotomy, skeletal traction or derotator osteotomy of femur shaft.

Conclusion: Pemberton acetabuloplasty shows good short term results regarding correction of the developmental dysplasia of the hip and also long term regarding prevention of secondary arthrosis.

However correct indication is crucial. Pemberton acetabuloplasty is associated 
with high correction potential and low complication rate.

Keywords: Pembeton, DDH, acetabuloplasty

\section{Internal Fixation vs. Total Hip Endoprothesis}

Aleksandar SAVESKI ${ }^{1}$, Aleksandar TRAJANOVSKI ${ }^{1}$, Teodora TODOROVA ${ }^{1}$, Rezeart DALIPI $^{1}$, Antonio GAVRILOVSKI ${ }^{1}$, Zoran BOZHINOVSKI $^{1}$, Ilir HASANI ${ }^{1}$, Simon TRPESKI ${ }^{1}$

${ }^{1}$ University Clinic for TOARILUC, - Skopje, MACEDONIA

\begin{abstract}
Introduction: Femoral neck fractures are frequent injuries in the patient population of every trauma center and have a high incidence in the general population. Paralleling trends of demographic forecasts, their incidence will continue to rise in the future. Especially in the elderly, femoral neck fractures represent a significant health care problem and have enormous impact on health insurance costs. Therefore, the appropriate treatment of femoral neck fractures is mandatory.

Aim: To measure the quality of life by SF36 score in patients, with femoral neck fractures treated with internal fixation and total hip endoprothesis. QoL of the patients will include the physical and mental health. Materials and methods: Retrospectively we evaluated data of 30 patients with femoral neck fractures divided in 2 groups based on the surgical treatment. The first group (youngsters) was treated with internal fixation and the second group (elder population) was treated with total hip endoprothesis. To measure the QoL of the patients we used SF-36 score.

Results: The patients treated with osteosynthesis showed delayed weight bearing and prevention of the femoral head comparing with the second group of patients who was treated with hip endoprothesis. The patients from the second group showed better QoL and early return to their everyday activities.
\end{abstract}

Conclusion: During the years, the outcome improved significantly due to the advancement in the osteosynthetic material and surgical techniques, also to the active rehabilitation. Many patients with femoral neck fracture will return in their homes and their daily activities just like before the injury.

Key words: femoral neck fractures, total hip endoprothesis, internal fixation

\section{Resolving Fractures of Proximal Femur in Geriatric Patients with Subtotal Endoprothesis in a Period of Two Years Our Experience}

\author{
Aleksandar TRAJANOVSKI ${ }^{1}$, Aleksandar \\ SAVESKI ${ }^{1}$, Rezeart DALIPI ${ }^{1}$, Teodora \\ TODOROVA ${ }^{l}$, Antonio GAVRILOVSKI ${ }^{1}$, Simon \\ TRPESKI ${ }^{1}$, Ilir HASANI ${ }^{1}$, Zoran BOZHINOVSKI ${ }^{1}$. \\ ${ }^{1}$ Univesity Clinic for TOARILUC - Skopje, \\ MACEDONIA
}

\begin{abstract}
Introduction: Hip fractures are among the most common type of fractures, especially in geriatric population. They are major cause of excess mortality, morbidity, health and social expenditure in elderly people.Bone is lost with advancing age in both men and women, leading to an increased risk of fractures after minimal trauma. The primary purpose of treatment and rehabilitation in the elderly after a hip fracture is to improve an individual's quality of life.
\end{abstract}

Aim: The management of patients with hip fractures, the surgical fixation with endoprothesis of the fracture and subsequent rehabilitation to allow the optimal recovery of functional capacity. Materials and methods: The study was performed at the University Clinic for TOARILUC in a two year period. 100 Patients with age over 70 , with proximal femoral fracture were included. All of the patients were treated surgically with implantation of subtotal endoprothesis. Results: The mean age of the patients at the time of operation was 77.4 years 
(range: $71-84$ years). At presentation were 60 female patients with osteoporotic fractures. All of our patients returned to their homes after the surgical treatment and hospitalization in good general condition, with early weight bearing and rehabilitation.

Conclusion: Advances in surgical techniques and instrumentation have allowed more effective life for the patients. It is important to underline that the principles and methods of functional restoration after a hip fracture should consider careful planning of the rehabilitation program for every individual patient and its implementation with respect to decisions made by the rehabilitation team.

Key words: Hip fractures, subtotal endoprothesis

\section{Cephalo-medullary Nailing of Inter Trochanteric Hip Fracture Surgical Technique and Pitfalls to Improve Results.}

Ledian FEZOLLARI ${ }^{1}$, Gjergji CAUSHI ${ }^{1}$, Artid DUNI $^{1}$, Vilson RUCI ${ }^{1}$, Ergys CAMI ${ }^{1}$, Korab SEJDINI $^{1}$, Serdi MEMINI ${ }^{1}$, Edvin SELMANI ${ }^{1}$, Zamir DEMIRAJ ${ }^{1}$, Guido BASSI ${ }^{2}$. Alfred GEGA ${ }^{1}$ ${ }^{1}$ Orthopaedic and Trauma Service, University Hospital of Trauma, Tirana, Albania

${ }^{2}$ Orthopeeadic Sevice, American Hospital, Tirana. Albania.

\footnotetext{
Abstract

Although the sliding hip screw has an excellent track record for stabilizing inter trochanteric hip fractures, more unstable fractures, especially those with disruption and comminution of the postero medial buttress, may be better suited for fixation with a cephalo medullary nail. Thereverse obliquity fracture pattern should be considered a contraindication to use the sliding hip screw, evidenced by failure rates. This fracture type should be stabilized with an intramedullary nail. Studies by Lindsey et al. and Davis et al. demonstrated multiple benefits of a cephalo medullary nail including: (1) providing stable head and neck fixation,
}

(2) allowing for controlled collapse and impaction of the fracture site, (3) decreasing the lever arm on the proximal fragment compared to the side plate by virtue of its intramedullary location, (4) providing excellent axial and rotational control, (5) allowing for early weight bearing, (6) allowing minimal dissection and "percutaneous" insertion, and (7) serving as a load-sharing implant device. This article aims to discuss and give a review of literature concerning cephalomedullary nailing of inter trochanteric hip fracture surgical technique.

Keywords: cephalo medullary nailing, inter trochanteric hip fracture, surgical technique, pitfalls

\section{Reverse Osteosynthesis with Distal Femoral Locking Compression Plate in Hip Periprosthetic Fractures}

Rezeart DALIPI ${ }^{1}$, Ilir HASANI ${ }^{1}$, Milan

SAMARDZISKI $^{1}$, Aleksandar SAVESKI ${ }^{1}$, Aleksandar TRAJANOVSKI ${ }^{1}$, Kamnar $V^{1}$, Antonio GAVRILOVSKI $^{1}$, Jahja $\mathrm{D}^{1}$, Teodora TODOROVA $^{1}$

${ }^{1}$ University Clinic for TOARILUC - Skopje, MACEDONIA

\begin{abstract}
Introduction: Periprosthetic fractures (PF) are considered as fractures associated with an orthopedic implant, whether a replacement or internal fixation device. The global incidence of all types of PF is constantly increasing due to growing number of primary joint arthroplasties and revision surgeries. Most frequently, periprosthetic fractures are result of low energy trauma, which has been shown to account for $75 \%$ of the fractures.

Significant comorbidities, osteoporosis/osteopenia, rheumatoid arthritis, and revision surgery are all contributing factors.
\end{abstract}

Aim: The aim of the report is to deepen the understanding of the technique in the surgically treatment of osteosynthesis the proximal femoral periprosthetic fractures 
with reverse application of distal femoral locking compression plate.

Material and methods: This report presents two cases with periprosthetic fractures treated with distal femoral locking compression plate applied in reverse technique. The surgeries of patients were performed sn the University Clinic for Orthopedics Surgery - Skopje. Both patients had type $\mathrm{B} 1$ and type $\mathrm{C}$ periprosthetic fractures according to Vancouver classification.

Results: The patients were discharged from the hospital two weeks after the surgery, in good general condition, satisfactory movements, both verticalised and they started weight bearing three days after the surgery.

Conclusion: Periprosthetic fractures around the femoral stem after total hip replacement (THA) are a significant and growing technical challenge for orthopedic surgeons, requiring proficiency in both THA and trauma care. The incidence of such fractures has a rising trend as the number of patients undergoing primary and revision THA increases. In general, postoperative recovery remains unpredictable and still there is a high risk of perioperative and postoperative complications after periprosthetic fracture surgery.

Keywords: Periprosthetic fracture, distal femoral locking compression plate, total hip replacement.

\section{10 Cases of Total Hip Replacement for Patients with Crowe type IV DDH}

Ledian FEZOLLARI ${ }^{1}$, Gjergji CAUSHI ${ }^{1}$, Artid DUNI $^{1}$, Vilson RUCI ${ }^{1}$, Ergys CAMI ${ }^{1}$, Korab SEJDINI $^{1}$, Serdi MEMINI ${ }^{1}$, Guido BASSI ${ }^{2}$ ${ }^{I}$ Orthopaedic and Trauma Service, University Hospital of Trauma, Tirana, Albania

${ }^{2}$ Orthopeadic Service, American Hospital, Tirana. Albania.

\section{Abstract}

Introduction: Hip dysplasia is one of the major conditions leading degenerative hip arthritis in early adulthood. Patients affected by high DDH have a unique and proper anatomy; both acetabulum and proximal femur are often associated with well-defined patterns. Moreover, the muscles and soft tissues around the hip are shortened and the capsule is thickened. The abductors insufficiency leads to a limp or to a frank Trendelenburg gait. The sciatic nerve is shortened predisposing to injury in case of limb lengthening greater than $4 \mathrm{~cm}$. Appropriate positioning of the acetabular component during total hip replacement in patients with hip dysplasia is often difficult.

In our study, 10 cases of high-level displacement type IV of Adult DDH Crowe classification were reviewed. Acetabulum was performed by implantation of the AVANTAGE RELOAD Acetabular Cup in paleoacetabul dual mobility with and without cement as the current consensus of acetabulum reconstruction recommends implantation of the cup in the true acetabulum in order to restore the lever arm of the gluteus medius and reduce hip joint reaction force. While in the femoral part we performed subtrochanteric shortening osteotomy maximally $4 \mathrm{~cm}$, which has been demonstrated to allow control of leg length, preservation of the abductor mechanism, and the correction of anteversion and implantation of Wagner Cone Prosthesis Hip Stem. Postoperative results have been pretty good. Patients were mobilized the third day after surgery. Only one case showed neuropraxis of the sciatica but recovered within 6 months. Walking balanced within 3 months in 6 cases of study. The study aims to give a review of literature and present a case series of total hip replacement for patients with crowe type iv DDH.

Keyword: Trans trochanteric osteotomies, Paavilainen osteotomy, Lesser trochanter osteotomy 
88. Catheter-Associated Urinary Tract Infections in Hip Fractures. Experience of Durres Regional Hospital.

Neritan MYDERRIZI ${ }^{1}$, Shkelqim FERKO ${ }^{2}$

${ }^{1}$ Service of Orthopaedy and Trauma. Durres Regional Hospital, Albania

${ }^{2}$ Service of General Surgery and Urology. Durres

Regional Hospital, Albania

\begin{abstract}
Background: Hip Fractures are most commonly in elderly people with an incidence that grows with age. These fractures have an annual mortality of 18$33 \%$ and hospital mortality of $2.7 \%$. Catheter-associated urinary tract infection is a common reality among these patients. $10 \%$ develop urinary tract infections.
\end{abstract}

Materials and Methods: This is a retrospective study including 210 patients with hip fractures during 2014-2016 timeframe in the orthopedic service of Durres Regional Hospital.

Results: The average age of 70.6 years (21-99 years old). 60 patients were catheterised before surgery, 150 during surgery, 45 patients extended the use of chatheter more than two days after surgery. Urinary tract infection was seen $15 \%$ in the group A, $8 \%$ in group B and $28 \%$ in group C.

Conclusion: Treatment of hip fractures needs not only a qualified orthopedic service, but also an intensive care team and a rehabilitation staff for post operatory period.

Extended use of indwelling urinary catheters postoperatively is associated with poor outcomes. Stable ostheosynthesis and early ambulation decrease the use of urinary catheter.

Keywords: hip fractures, urinary tract infections, catheter-associated

\section{Damage Control Surgery in Abdominal Trauma and Intraabdominal Catastrophes; Need to New Approaches}

\author{
Rifat LATIFI ${ }^{1}$ \\ ${ }^{1}$ New York Medical College, School of Medicine \\ Director, Department of Surgery, Westchester \\ Health Network, Department of Surgery, New York \\ Medical College. Valhalla, NY, USA.
}
Abstract
Damage control surgery (DCS) concepts and techniques have been part of our clinical armamentarium in trauma for decades, but recently DCS has expanded to other surgical disciplines: emergency general surgery; neurosurgery (craniectomies); orthopedics surgery, particularly for trauma; thoracic surgery; vascular surgery; liver transplant surgery; and other surgical fields. DCS is characterized by termination of the surgical intervention after control of bleeding and contamination, followed by hemostatic resuscitation and definitive management. What technique and approach is used depends on pathology at hand. It is a staged approach that takes into consider- ation the physiologic reserves of the patient, and it is designed to avoid or treat the lethal triad of hypothermia, acidosis, and coagulopathy. The decision to perform DCS is complex and requires solid knowledge of physiology of the patient as well as the associated injuries or comorbid disease. Moreover, it takes a complete situational awareness about the patient, his/ her physiology, all end-point resuscitation, and surgical team dynamics and skills.Indication for DCS are hemodynamic instability, hypothermia ( $<35 \mathrm{C})$, coagulopathy, severe metabolic acidosis ( $\mathrm{pH}<7.2$ or base deficit $>8$ ), multiple injuries, massive transfusion requirements ( $>10$ units packed red blood cells), and long operative time (>90 min) for trauma or emergency are basic indication for abbreviating the procedure and some sort of temporal abdominal closure. 


\section{Management of Abdominal Vascular Injury}

Thomas B. WHITTLE ${ }^{1}$

${ }^{1}$ Vascular Surgeon Heartland Vein \& Vascular Institute, Omaha, NE, Lincoln Surgical Group Lincoln, NE, USA

\section{Abstract}

The vast majority of abdominal vascular injury is penetrating trauma, usually GSW. There is frequent association of other intraabdominal injuries. This combination is often lethal, and less than $50 \%$ of patients survive. Patients often present in hypovolemic shock with hypothermia, acidosis, and coagulopathy. Emergency Department Thoracotomy (EDT) has been advocated by some, but results are poor. Others recommend avoiding EDT, and looking for clues of vascular injury on physical exam. Injury above or involving SMA presents with abdominal pain and distention. Pararenal involvement presents with hematuria. And injury to the infrarenal aortoiliac vessels presents with bilateral or unilateral lower extremity ischemia. Resuscitation includes IV access, blood product replacement, midline abdominal incision, evacuation of intraperitoneal blood, and intraabdominal packing. This allows anesthesia time to catch up. Systematic exploration of the zones of injury and identifying associated injuries is then carried out. The decision to explore a specific vascular hematoma depends upon location and type of injury (penetrating or blunt). Prioritizing repair is important after active hemorrhage is controlled. Kidneys are most sensitive to prolonged ischemia, followed by bowel, and then LE ischemia. Exposure techniques with proximal and distal control depends upon the zone of injury. These include supraceliac or infrarenal aorta exposures, and left or right medial visceral rotations. Repair method depends upon extent of vascular injury, degree of contamination, time of ischemia, and patient stability. Ligation or shunting with return to the OR after stabilization should be considered. Risk of intraabdominal compartment syndrome or LE compartment syndrome is significant.

\section{Management of Severe Hepatic Trauma}

Fausto CATENA ${ }^{1}$

${ }^{1}$ Professor of general surgery, Chief Department of Emergency and General Surgery Parma University Hospital ITALY.

\section{Abstract}

The severity of liver injuries has been universally classified according to the American Association for the Surgery of Trauma (AAST) grading scale. In determining the optimal treatment strategy, however, the haemodynamic status and associated injuries should be considered. Thus, the management of liver trauma is ultimately based on the anatomy of the injury and the physiology of the patient. Our aim is to present the World Society of Emergency Surgery (WSES) classification of liver trauma and the management Guidelines

\section{Management of Anterior Penetrating Abdominal Injuries. Physical Examination vs Radiologic Imaging vs Diagnostic Laparoscopy}

Orhan ALIMOGLU ${ }^{1}$

${ }^{I}$ Department of General Surgery, School of Medicine, Istanbul Medeniyet University, Istanbul,Turkey
Abstract
Anterior penetrating abdominal injuries are frequently encountered conditions in cases of trauma \& emergency surgery. Inthe 1960 's, routine exploratory laparotomies were widely performed in such cases. The therapeutic approach has changed over the years from routine laparotomy to non- operative treatment.Currently, as elective conservative treatment for penetrating anterior abdominal injuries is an increasingly recognized approach. 
Although emergency laparotomy is still recommended for patients who either are hemodynamically unstable, or present with signs of peritonitis at the initial evaluation, conservative approach is preferred for the remaining cases who lack these findings. However, there's still controversy in concern of how the conservative management should be decided on, or performed. Three methods are commonly used for the decision of conservative treatment which are physical examination along with necessary laboratory tests, radiologic imaging techniques including ultrasonography and computerized tomography scan, and diagnostic laparoscopy. It has been reported that the accuracy for ultrasonography and computerized tomography scan were $80 \%$ and $77 \%$ respectively, whereas this rate for physical examination was found to be 95\%. On the other hand, performing diagnostic laparoscopy for all patients is not considered as cost-effective due to the invasive nature of this intervention and its probable complications.

In conclusion, a selective conservative approach based on physical examination, simple laboratory studies (hemogram, creactive protein) and clinical follow-up in penetrating anterior abdominal stab injuries is a simple, cost-effective, reliable and effective treatment method.

Keywords: anterior penetrating abdominal injuries, physical examination, radiologic imaging, diagnostic laparoscopy.

\section{The Rate of Success of the Conservative Management of Liver Trauma in Albania}

Skender BUCI ${ }^{1}$, Myftar TORBA ${ }^{1}$, Sokol RUCI ${ }^{1}$, Dorina SHTJEFNI ${ }^{1}$, Elona MARKECI ${ }^{1}$ General Surgery Service, University Hospital of Trauma, Tirana, Albania.
Albania. The percentage of success was $58.7 \%$.

Methods: This study was conducted prospectively from January 2009 to December 2012. We analyzed 173 patients admitted to our hospital with liver trauma. Liver injuries were evaluated according to the American Association for the Surgery of Trauma and the World Society of Emergency Surgery classification, while the anatomic gravity of the associated injuries was defined using the Injury Severity Score system. The potential mortality was estimated with the Revised Trauma Score.

Results: Out of the 173 patients with liver trauma, $83.2 \%$ were male. The main cause of liver trauma was motor vehicle crashes $(50.9 \%)$. Blunt trauma was the cause of liver injury in 129 cases $(74.6 \%)$, and penetrating trauma occurred in 44 cases (25.4\%). Initially, the decision was to manage 88 cases $(50.9 \%)$ via the conservative approach.

Of these, 73 cases $(42.2 \%)$ were successfully treated with conservative treatment, while in 15 cases $(17.2 \%)$, this approach failed. The success rate of conservative treatment by grade of injuries was as follows: grade I (38.4\%), grade II $(30.1 \%)$, grade III $(28.8 \%)$, and grade IV $(2.7 \%)$. The overall mortality rate of liver trauma was $13.2 \%$.

Conclusions: The likelihood of success in using conservative treatment had a significant correlation with the grade of liver injury and associated intra-abdominal injuries. The limited hospital resources and low level of consensus on conservative treatment had a negative impact on the level of success.

Keywords: Liver trauma, Grade of injuries, Conservative treatment, Success

\footnotetext{
Abstract

Background: The conservative treatment of liver trauma has made important progress over the last 10 years at the University Hospital of Trauma in Tirana,
} 


\section{Emergency System Evolution in Albania}

Skënder BRATAJ ${ }^{1}$

${ }^{I}$ National Center of Medical Emergency, Tirana, Albania

\section{Abstract}

A fully functional integrated system of the Coordination Unit of the National Center of Medical Emergency was launched for the first time in Albania, in 3 April 2017. A functional emergency system is the first step towards an advanced health system. Our mission is to save life and to guarantee its quality. The Emergency Service $127 / 112$ guarantees immediate response to any medical emergency throughout the territory of the Republic of Albania. We respond at any request for medical help by arriving immediately after the call at the scene, where the medical team does the assessment, treatment, stabilization of vital parameters and transportation to the appropriate hospital. This mission is carried out through a structured organization and availability of all the financial, legal, human resources, and the necessary equipment and tools. We guarantee the life quality of our patients only with the professional preparation and the standardized protocols, the continuous collaboration with national and international scientific associations, with a constant evidence-based verification and updating. This article aims to describe and discuss the coordination unit of the National Center of Medical Emergency, its role, tasks, purposes and evolution throughout the years.

Keywords: National Center of Medical Emergency, Albania, evolution, emergency, 112, 127

\section{How to Manage a Case with Multiple Injuries in Prehospital and Hospital Premises}

Basri LENJANI ${ }^{1}$, Premtim RASHITI ${ }^{1}$, Gani SHABANI $^{1}$, Esen UYSAL ${ }^{1}$, Elfije KRASNIQI ${ }^{1}$, Erza VOCA ${ }^{1}$, Berat LENJANI ${ }^{1}$, Kledisa HARIZI ${ }^{1}$, Dardan LENJANI ${ }^{1}$

${ }^{1}$ Emergency Clinic, University Clinical Center of Kosovo, Pristine, Kosovo.

\section{Abstract \\ Introduction: In the University Clinical Center of Kosovo the Tertiary Emergency Clinic on average require emergency medical help 1-3 cases of multiple injuries each day. Of these injured, some come directly and many of them are referred to by regional hospitals. Multiple injuries pose problems, challenges in managing multiple-injured injuries. \\ The purpose of this paper is to identify problems, different complaints, advice and possible solutions to managing injured multiple injuries, reducing morbidity, disability and mortality.}

Material and Method: The research is of a retrospective type for a period of 8 months from January to August 2018. All data on multiple injured sufferers have been studied and researched the problems posed during the management of multiple injured injuries ranging from step one to step six. Results. From the investigated data, the young age, and especially the males, were mostly injured by traumas and road traffic accidents as the main etiologic factor of multiple injuries. Injuries were more than 2 to 3 system injuries, posing many problems in managing multiple-injured seniors.

Conclusions. A well-trained and welltrained medical team is the key to effective management of multiple injured people, minimizing the problems encountered during the management steps.

Words. Multiple injuries, injuries, emergency clinic, triad. 
96. The ECG in Emergency Medicine

\section{Skënder BRATAJ ${ }^{1}$}

${ }^{I}$ National Center of Medical Emergency, Tirana, Albania

\section{Abstract}

The electrocardiogram is the recording and graphic reproduction of the electrical activity of the heart at various stages of its activity. It is a fundamental and irreplaceable diagnostic tool, but its interpretation should be linked to the symptoms and the history of the patient's illness. The electrocardiogram (ECG) is the ideal diagnostic test at the emergency medicine department. It is non-invasive, inexpensive, easy to use, and it yields a wealth of information. All emergency physicians interpret multiple ECGs every day and the most critical decisions related to patients with chest pain, dyspnea, or even shock, are based on ECG interpretation at the bedside. However, although the "high profile"' disease states such as acute coronary syndrome classically are linked with this indispensable tool, we use ECG as an important diagnostic test in other diseases too.

Although traditionally the ECG is thought of as a cardiologist's tool, the possibility of making real-time assessments of patients, has converted it into the domain of any medical practitioner, emergency physician, internist or family practitioner. All the physicians and nurses at the Emergency Department must become very comfortable with the many facets and subtleties of ECG interpretation, furthermore they should be expert in the urgent and emergent interpretation of the ECG.

In this issue, we examine the ECG in traditional and nontraditional realms. Diagnosis of dysrhythmia and acute coronary syndromes is an obvious focus of this text. We also discuss electrocardiographic manifestations of non-coronary disease, both cardiac and systemic.

Keywords: ECG, emergency medicine, diagnostics
97. Access to Managing a Case with Traumatic Cardiac Arrest and Emergency Treatment in the Prehospital and Hospital Environment

Basri LENJANI ${ }^{1}$, Premtim RASHITI ${ }^{1}$, Gani SHABANI $^{1}$, Esen UYSAL ${ }^{1}$, Elfije KRASNIQI ${ }^{1}$, Berat LENJANI ${ }^{1}$, Kledisa HARIZI ${ }^{1}$, Dardan LENJANI $^{1}$

${ }^{1}$ Emergency Clinic, University Clinical Center of Kosovo, Pristine, Kosovo.

\begin{abstract}
Traumatic cardiac arrest is the breakthrough of heart work which is terminated due to open or penetrating injuries to the chest. Traumatic cardiac arrest is a medical emergency which will always result in death if no immediate medical care is provided and survival without neurological complications is rare. There are no definitive protocols on how to manage traumatic cardiac arrest, but some victims benefit from emergency thoracotomy to gain access and repair damage from chest injuries. Traumatic cardiac arrest is a complicated form, often confusing with the Advanced Cardiac Life Support protocols, in the broadest sense that the emergency team first needs to determine the cause of traumatic cardiac arrest and change, considering hypovolemic and hemorrhagic shock to due to penetrating injuries. As all traumas are treated by a specialized medical team who are trained using (ATLS). The survival rate of victims has improved significantly if the injured gets final treatment (operation or reperfusion) within an hour of an accident and this critical time principle is usually known as the "golden hour". Medical hospital transport often becomes inaccessible to the local facility when the injured party requires advanced medical care.
\end{abstract}

Keywords. Traumatic cardiac arrest, Responder. ACLS. ATLS, medical emergencies. 


\section{Emergency Treatment in Himara Tragic Accident (Albania)}

Edlira KUKELI ${ }^{1,2}$, Valdete KALOSHI ${ }^{1,2}$, Valbona ZEKA $^{1,2}$

${ }^{1}$ University Hospital of Trauma, Tirana, Albania

${ }^{2}$ University Hospital Center " Mother Tereza”

Tirane, Albania

\section{Abstract}

Background: There is an increasing of road accidents during recent years. On 21 May 2012 a tragic accident near Visha's hill (Himare) happened, when a bus plunged 80 metres off a cliff. The accident killed 11 students and injured 22 others. The ambulances and helicopters rescue were sent to assist and bring them to medical resources. The University Hospital of Trauma provided medical care and treatment of the wounded and injured. Aim: This article aims to discuss the medical response of emergency department, management and transport of injured to the tertiary care hospital.

Materials and Method: The retrospective study was conducted during the period of time (2012 -2013). It is based on the obtained hospital data of wounded (medical records).

Results: 20 (females) of the evacuated people were treated at the University Hospital of Trauma. The two remaining injured were dead during their helicopter transport. The patients were hospitalized in intensive care $45 \%$, traumatology $15 \%$, neurosurgery $22 \%$, general surgery $12 \%$, and urology $6 \%$. The rescue work was performed well...

Conclusions: Emergency Departament is a sensitive area of health care. Triage is the most important mission of emergency management. We should provide an effective strategy of emergency response improving based in institutional cooperation and lessons learned.

Keywords: University Hospital of Trauma, Albania, Himara, accident, management
99. Retrospective Study by Bristow Technique in the Treatment of Habitual Scapulo-Humeral Dislocation

Ilia MAZNIKU ${ }^{1}$

${ }^{I}$ Faculty of Technical Medical Sciences, University of Elbasan "A. Xhuvani", Elbasan, Albania

\begin{abstract}
Aim: To evaluate the functional outcomes of the Bristow procedure in patients with recurrent anterior glenohumeral instability. Materials and methods: Personal clinical records of 42 patients with 45 operated shoulders were reviewed retrospectively. Patient age at time of first dislocation, injury mechanism, and number of recurring dislocations before surgery were recorded. The overall function and stability of the shoulder was evaluated.
\end{abstract}

Results: Thirty-five cases or $78 \%$ of the scapulo humeral instability series have been traumatic. The average number of repeated dislocations was from 9 to 12 reports A patient, a 25-year-old male, had 17 episodes of shoulder outbreaks in one day. The average of 46 months of prosecution. After the surgical procedure we have not encountered any relapse. In four patients (9\%). A fibrous consolidation of the corrosion process on the front lip of glenoidales was observed. Only two had clinical signs of pain and discomfort. One of them was reworked for removing screws with very good postoperative results. The overall functional end result has been good. Patients enjoy full functional supine activity, confidence in using it.

Conclusions: The Bristow procedure is a very good surgical treatment for recurrent anterior instability of the glenohumeral joint. The shoulder function after the intervention is complete and prominent. Although there is a limitation on the outer shoulder curl.

Keywords: Bristow technique, habitual scapulo-humeral dislocation, treatment 
100. Clinical Outcomes in

Internal Synthesis of the Humerus

Head Split Fractures, $A$

Randomized Study.

Dritan TODHE ${ }^{1}$

${ }^{1}$ Orthopedic and Trauma Service, University

Hospital of Trauma, Tirana, Albania

\begin{abstract}
Background: Humerus head split fracture is rare and the importance of correct diagnosis of the type of the fracture is crucial in order to achieve good result. The aim of this study is to discuss theclinical outcomes in internal synthesis of the humerus head split fractures as well as to give a review of literature.
\end{abstract}

Materials and Methods: A retrospective study of humerus head split fracture operated during 2014-2015 in Tirana Albania, classified according the severity of fracture and age.

Results:We have a series of 15 patients < 50 years, who underwent locking plate fixation. We noticed that in a mean of 34 months follow up, 12 of them had united. No osteonecrosis or nonunion was seen in simple fractures (5 patients). In complex fractures (10 patients), osteonecrosis was seen in 4 patients, nonunion in 2 patients, and gleno-humeral arthritis in one patient. We concluded that complex fractures are associated with higher rates of nonunion, avascular necrosis and inferior shoulder function. We also considered the RSA in the failed cases

Conclusion: Although we prefer to consider internal fixation first, we suggest that RSA is to be considered in the first signs of failed, otherwise the postoperative fibrosis will lead to bad functional results. Keywords; Humerus head split fracture, internal synthesis, reverse shoulder prosthesis

\section{Minimally Invasive Surgical Treatment of Fractures of the Neck and Head of the Radius with ESIN Method}

Marjan KAMILOSKI ${ }^{1}$, Lazar TODOROVIC ${ }^{1}$, Simon TRPESKI ${ }^{1}$, Jetmir ZIBERI ${ }^{1}$, Lazo JOVCHESKI ${ }^{1}$, Slavica SHUPERLISKA ${ }^{1}$. Anila RACAJ $^{1}$, Zllatko ALEKSOVSKI ${ }^{1}$.

University Clinic of Pediatric Surgery Skopje, Macedonia

\section{Abstract}

Fractures to the neck and head are represented by $1-2 \%$ of all child age fractures. These types of fractures were described for the first time in $1803 \mathrm{yr}$. This retrospective study analyses and presents the results from the treatment of patients with these fractures in the period from 2010 to 2015. The types of fractures are classified under the Judget classification. The goal of our study is to evaluate the advantage of using the minimal invasive surgical treatment with fractures of the head and tne neck of the radius type III and IV. Two surgical techniques were used: 42 patients were treated with an open and bloody reposition and transcondylar fixation, while 32 patients were treated with the ESIN method. The choice of the surgical technic was random. This study has observed the inclusion and exclusion criteria. For the evaluation and sorting of the received results we used the "Broberg and Morrey score system". The received results from the two examined groups were in the scope of expectedly good results. Following the positive trends in child traumatology, which prefers the minimal invasive methods, our study also, showed better results in this group. 


\section{Proximal Humerus \\ Fractures Treatment \& \\ Management}

Dorian HABILI ${ }^{1}$

${ }^{1}$ Orthopaedic and Trauma Service, University

Hospital of Trauma, Tirana, Albania

\begin{abstract}
Proximal humerus fractures are

challenging to treat, as even low-energy

mechanisms of trauma may result into

complex fracture patterns, especially in the

elderly population. They make 5-9\% of all

fractures in our clinical practice. Proximal

humerus fractures have a dual age

distribution, occur either in young people

following high energy trauma or in those

older than 50 years with low velocity

injuries like simple fall.
\end{abstract}

In the elderly population, most of these

fractures are related to osteoporosis. They

have a bimodal age distribution. Proximal

humeral fractures are the third most

common fracture in elderly patients and

pose an important socioeconomic problem.

More than $70 \%$ of the patients with these

fractures are older than 60 years of age,

and $80 \%$ are women.

Complex three- and four part fractures

account for $>50 \%$ of cases in patients older

than 60 years. The major part of these

fracture are treated conservatively, with

approximately $20 \%$ of cases requiring operative management.

Nevertheless, the treatment of choice remains a matter of controversy. There is no single method to impose itself as the gold standard of treatment. Proximal humerus fractures present a unique challenge to surgeons as surgical indications are poorly defined in the literature. Historically, studies are complicated by a lack of comparison groups and nebulous definitions of surgical indications. At this time, it is not clearly defined exactly which patient and fracture type perform better with surgical intervention. Surgeons should always consider the patient and their expectations prior to recommending surgery, as satisfactory results have been shown from conservative care even in the face of displacement and comminution. This article aims to give a review of literature and discuss the treatment and management modalities of proximal humerus fractures. Keywords: proximal humerus fractures, management, treatment, literature

\section{Multidisciplinary Surgical Approach in Extensive Trauma Injury of Upper Extremity - Case Report}

Lazar TODOROVIC ${ }^{1}$, Ilir HASANI ${ }^{2}$, Anila

RACAJ ${ }^{1}$, Marjan KAMILOSKI, Slavica SHUPERLISKA $^{1}$

${ }^{1}$ University Clinic of Pediatric surgery, Skopje, $R$. Macedonia

${ }^{2}$ University Clinic of Traumatology, Skopje, $R$. Macedonia

\section{Abstract}

Introduction: Polytraumatized an 9-yearold patient, in a hemorrhagic and traumatic shock with severe laceration injury of the left upper extremity and early sings for acute critical ischemia, was admitted in our emergency department

Material and Methods: Surgery was initiated within the first hour of hospital admission. Exploration revealed highly contaminated wound, extensive soft tissue damage, severe muscle laceration, axillary artery lesion, thrombosed axillary vein, neuropraxia of the brachial plexus and open humeral shaft fracture grade III according Gustilo- Anderson classification. The axillary artery lesion was replaced with a reversed saphenous vein interposition graft and the axillary vein thrombus was removed using Fogarty catheter. The open humeral shaft fracture was stabilized with external fixation ostheosynthesis. After meticulous and radical injury zone excision was completed, soft tissue coverage and tension free approximation of the muscle and skin was achieved. Pre-and intraoperative broadspectrum antibiotics were used. Results: Postoperative follow up was satisfactory with acceptable functional and 
aesthetic outcome. Complete healing of the humeral shaft fracture was noticed after 18 weeks. Rehabilitation program was started after humeral fracture was stable.

Conclusion: The treatment of this kind of injuries require multidisciplinary surgical approach that includes traumatology surgeons, vascular surgeons and plastic surgeons

\section{Rehabilitation of Shoulder after Rotator Cuff Repair}

Manushaqe SARAÇI ${ }^{1}$, Luljeta STANAJ ${ }^{1}$

${ }^{1}$ Rehabilitation and Physiotherapy Service, University Hospital of Trauma, Tirana, Albania

\section{Abstract}

Background: The rotator cuff damage is the most common cause after road accidents. Rehabilitation after the arthroscopy of the rotator cuff is very important, which leads to: Pain relieving, ROM recovery, shoulder strength.

Aim: To compare the effect of early versus delayed motion in clinical outcomes of patients who have undergone arthroscopic rotator cuff repair.

Materials and Methods: This is a prospective study, involving 36 patients who were treated at our service during March 2016 -March 2018. Average age 55+-5years old, M/F 14/22 (19 with right arm and 17 with left arm). 18 patients started early motion protocol 2-3days after surgery (First group), 18 patients started the $28^{\text {th }}$ day after surgery (second group). The rehabilitation protocol was applied with 4 phases: Phase I(weeks 1-6), phase II(weeks 6-12), phase III(weeks $12^{+}$), phase IV(months $4^{+}$). For measurements we used VAS scale, goniometry, MMT at the end of each phase of rehabilitation $\left(1^{\text {st }}\right.$ day, weeks 3,6,12, and months 6,12 ). Criteria for progression in phase: 1 Minimal pain, inflammation and swelling 2-Muscular strength $\geq 4 / 5$ throughout. Results:1-There was significant difference in pain score. In the first phase of rehabilitation $89 \%$ of patients of the first group according to VAS score were in 6 points, while $85 \%$ of patients of the second group were in 3 points in the same phase. 2-There were differences in the VAS scores of the first phase of rehabilitation between the two groups. 3-At a later time functional scores, active motion and shoulder strength had no differences. Conclusions: The two groups had differences in VAS score at first phase of rehabilitation while functional scores, active motion and shoulder strength hadn't. Keyword: Rotator cuff repair, arthroscopy, rehabilitation.

\section{Shock Index in Abdominal Trauma}

Ayman El-MENYAR ${ }^{1}$

${ }^{1}$ Director of clinical Research in Trauma and vascular surgery in Hamad General Hospital, Doha, Qatar

\section{Abstract}

Shock index (SI), is considered as a rapid predictable indicator of hemodynamic instability in trauma patients. Herein, we aimed to investigate the predictive value of shock index in patients with abdominal trauma. Patients were classified into group I $(S I<0.8)$ and group II $(\mathrm{SI} \geq 0.8)$. Out of 1199 patients with abdominal trauma requiring hospital admission, $49 \%$ had $\mathrm{SI} \geq$ 0.8. In comparison to lower SI group, patients with $\mathrm{SI} \geq 0.8$ were significantly 6 years younger, had greater ISS, and received more blood transfusion and MTP, and had more exploratory laparotomy and higher mortality, $\mathrm{p}=0.001$. After adjusting for age and sex, ISS, GCS and abdomen AIS, presence of high SI was independent predictor for exploratory laparotomy (aOR 2.25) and MTP (aOR 8.07). A prospective study could be useful to assess weather higher SI has practical implications for risk stratification for the need of early MTP and surgical intervention for abdominal injuries before incorporating it as a standard of care clinical investigation. 
106. Management of Spleen

\section{Trauma}

Fausto CATENA ${ }^{1}$

${ }^{1}$ Professor of general surgery, Chief Department of Emergency and General Surgery Parma University Hospital ITALY.

\section{Abstract}

Spleen injuries are among the most frequent trauma-related injuries. At present, they are classified according to the anatomy of the injury. The optimal treatment strategy, however, should keep into consideration the hemodynamic status, the anatomic derangement, and the associated injuries. The management of splenic trauma patients aims to restore the homeostasis and the normal physiopathology especially considering the modern tools for bleeding management. Thus, the management of splenic trauma should be ultimately multidisciplinary and based on the physiology of the patient, the anatomy of the injury, and the associated lesions. Lastly, as the management of adults and children must be different, children should always be treated in dedicated pediatric trauma centers. In fact, the vast majority of pediatric patients with blunt splenic trauma can be managed nonoperatively. Our aim is to present the World Society of Emergency Surgery (WSES) classification of splenic trauma and the management guidelines.

\section{Algorithm Based Evaluation of Traumatic Diaphragm Injuries}

Orhan ALİMOGLU ${ }^{*}$

${ }^{1}$ Department of General Surgery, School of Medicine, Istanbul Medeniyet University, Istanbul,Turkey

\section{Abstract}

Diaphragmatic injuries may be en countered following either blunt, or penetrating abdominal trauma. In the past, when conservative approach was not utilized and routine laparotomies were performed, the evaluation and mangement of diaphragmatic injuries could generally be carried out accurately via the exploration of the diaphragm during laparotomy. Currently, since conservative approach for selected cases has widely been accepted, the diagnosis of diaphragmatic injuries has become an important issue.

In blunt trauma patients, diaphragmatic injury is rarely encountered. Nevertheless, in cases of blunt trauma, it should always be kept in mind that the evaluation of the diaphragm is as necessary as the evaluation of other organs and structures. Therefore, a multi-slice computerized tomography scan focused on the diaphragm is a valuable diagnostic tool, which also is usually sufficient for the management of such cases.

In penetrating trauma patients, left and right thoracoabdominal injuries are evaluated as separate entities. For rightsided injuries, the risk of the diaphragm to be affected is relatively low and follow-up is usually adeqaute. However, penetrating left thoracoabdominal injuries are accompanied by diaphragmatic injuries in $25-30 \%$ of cases, about $30 \%$ of which later develop into diaphragmatic hernias. In suchcases, diagnostic laparoscopy for evaluating diaphragm injuries is the preferred diagnostic approach as it also allows therapeutic measures, as well, when necessary. This presentation aims to discuss the algorithm based approach to traumatic diaphragm injuries.

Keywords: diaphragmatic injuries, trauma, evaluation, algorithm...

\section{Splenic Injuries After Blunt Abdominal Trauma}

Rexhep SELMANI ${ }^{1}$, Goran BEGOVIC ${ }^{1}$, Zoran KARADZOV $^{1}$, Gjorgji JOTA ${ }^{1}$, Qemal RUSHITI ${ }^{1}$, 
Andrijan KARTALOV ${ }^{2}$, Tatjana SPIROVSKA ${ }^{2}$, Marija ATANASOVA ${ }^{3}$

${ }^{1}$ University Clinic for Digestive Surgery, Skopje, Macedonia

${ }^{2}$ University Clinic for Anesthesiology and Intensive Care, Skopje, Macedonia

${ }^{3}$ General Hospital Veles, Macedonia

\section{Abstract}

Injuries to the spleen are one of the most common injuries in abdominal trauma. Unrecognized injury can be a cause of preventable traumatic death.

We analyzed in our institution the data of 110 cases with blunt abdominal trauma over a five year period (2013-2017). The mean age of patients was 55,4. Of the 91 patients $(83 \%)$ is male. Isolated blunt abdominal injury with lesions of the spleen is 6 , the other cases is with injuries in others systems (polytrauma).87 (79\%) was operated and 23 cases was treated conservative, the 75 cases was operated on the first day. The mortality was 15 cases $(13 \%)$ of the total number.

The blunt abdominal trauma remains one of the most common causes of spleen injury. The clinical status of the patient, the degree of spleen injury and associated injuries are the guidelines on the type of approach and treatment in patients with spleen injury. Today the blunt abdominal trauma with severe spleen injury is the most frequent indication for splenectomy.

Keywords: Blunt abdominal trauma, splenic injury, splenectomy.

\section{The Importance of Laparoscopy in the Evaluation of Intraabdominal Traumatic Lesions}

Ymer DURMISHI ${ }^{1}$, Philippe KONSTANDINIDIS ${ }^{1}$, Pascal GERVAZ ${ }^{1}$, Sami BYTYCI $^{2}$, N. ELSHANI ${ }^{2}$, Adrian HOTI ${ }^{3}$

${ }^{1}$ Surgery Service at Geneva University Hospital, Switzerland
${ }^{2}$ University Clinical Center of Kosovo, Pristine, Kosovo

${ }^{3}$ University Hospital Center "Mother Theresa", Tirana, Albania.

\begin{abstract}
Introduction: The first steps in the use of laparoscopy for abdominal emergencies were very slow. Nowadays, with the standardization of criteria and methodologies the evaluation by means of laparoscopy of intraabdominal lesions but also of their treatment has been accepted as a sure and efficient technique. Also the technical progress in the field of laparoscopy as well as the formation of teams constituted a significant turn in the use of these techniques. Even if this method cannot detect all the lesions, it helps us generally to evaluate the importance of traumas and to choose the type of therapy in a rational way.
\end{abstract}

Materials and Methods: For the present study, we have used the data of various medical centers as well as various medical reviews which treat this subject. We have used as well the data bases of different surgical and scientific associations.

Conclusion: Laparoscopic interventions for the evaluation and treatment of traumatic intraabdominal lesions in stable hemodynamic patients have proved to be a sure and successful method. According to the indications found, we consider that in the future we need more multicentrical and randomized studies. The numbers show that more than $50 \%$ of laparoscopic interventions have not only served as diagnosis, but also as treatment. Death occurs in $10-15 \%$ of traumatic abdominal lesions. The benefits of laparoscopy are largely documented in the treatment of abdominal emergencies in the field of evaluation as well as in the field of consumption of drugs. It decreases the number of days spent in the hospitals as well as the number of complications after surgery and accelerates the return of patients to work.

Key words: Laparoscopy, abdominal trauma, abdominal wounds, therapy and diagnosis 
110. Trauma and Endoscopic Surgery

Ilir SHANI ${ }^{1}$

${ }^{1}$ Surgery Department, Regional Hospital of Durres, Albania
Abstract
The introduction of laparoscopy has provided trauma surgeons with a valuable diagnostic and, at times, therapeutic option. The minimally invasive nature of laparoscopic surgery, combined with potentially quicker postoperative recovery, simplified wound care, as well as a growing number of viable intraoperative therapeutic modalities, presents an attractive alternative for many traumatologists when managing hemodynamically stable patients with selected penetrating and blunt traumatic abdominal injuries.
At the same time, laparoscopy has its own unique complications associated with diagnostic and therapeutic laparoscopy in trauma, focusing on practical aspects of identification and management of laparoscopy-related adverse events.

Keywords: laparoscopy, trauma, endoscopic surgery

\section{Imaging Diagnosis and Follow Up of Traumatic Pancreatic Injuries}

Sonja (Saraçi) BUTORAC ${ }^{1}$, Vidi DEMKO ${ }^{1}$, Admir MUSTAFA $^{1}$, Kujtim ALIMERI ${ }^{1}$, Gezim GURI ${ }^{1}$, Artan GJIKA ${ }^{1}$, Xhesika XHETANI ${ }^{3}$, Natasha MERKO $^{4}$, Agron DOGJANI ${ }^{2}$

${ }^{1}$ Department of Radiology, University Hospital of Trauma, Tirana, ALBANIA.

${ }^{2}$ Department of Surgery, University Hospital of Trauma, Tirana, ALBANIA.

${ }^{3}$ Resident of Radiology, University of Medicine, Tirana, ALBANIA.

${ }^{4}$ Nurse Service, University Hospital of Trauma, Tirana, ALBANIA.

\section{Abstract}

Introduction: Pancreatic trauma is a rather uncommon injury, but if present it is generally associated with multiple intraabdominal lesions, thus carrying significant morbidity and mortality.
Prompt diagnosis is therefore crucial, but, on the other hand, early diagnosis of pancreatic trauma can be challenging because of lack of correlation between the initial clinical features, radiological and laboratory findings, and the severity of the trauma. In other cases, specific injury patterns in the pancreas may have variable expression at the early 'panscan', especially as such examination does not include a dedicated protocol for scanning the pancreas.

Cases Report: We are going to report five cases of high-grade pancreatic injury, two of which upon the imaging diagnosis and grading underwent surgical intervention with Roux-en-Y pancreatico-jejunostomy. Peritoneal lavage and large drainage of the lesser and greater peritoneal cavity was performed. In addition to other examinations, the patients underwent several CT and/or MRI examinations to follow-up on the surgical outcomes and possible morbidities; especially as in addition to evaluation of parenchymal injuries, diagnosis of the pancreaticojejunostomy disruption is important in preventing delayed complications.

Conclusions: We add that imaging plays a crucial role in the timely diagnosis of pancreatic injury and its accurate grading, thus playing an important role in patient's management.

Keywords: Pancreatic trauma, pancreatic injury, initial abdominal CT

\section{Computed Tomography as an Adjuvant Imaging Modality in Managing of Abdominal Trauma.}

\author{
Albana SHAHINI \\ Radiology services American Hospital No 1 \\ Tirana, ALBANIA
}

\section{Abstract}

Introduction: In our everyday practice in Radiology Department facing with abdominal trauma is not a rare condition.

Abdominal computed tomography (CT) can assist in the decision to perform surgery 
or laparotomy in patients following blunt trauma to the abdomen.

Matherial and Methods: Based on the data gathered from January 2013- December 2017 we evidenced 96 patients with blunt abdominal trauma underwent evaluation with CT.

Results: Abdominal injuries were detected in 24 patients (25\%). These included: traumatic damage of solid viscera in 18 patients ( 10 splenic, 7 hepatics, 1 renal); four injuries to hollow viscera (three small bowel, and one rupture of the urinary bladder); and 2 skeletal injuries (fractures of the pelvis). Injury to solid viscera was categorized as minor in $44(46 \%)$, moderate in $15(16 \%)$, or severe in $37(38 \%)$ according to an assessment of the percentage of parenchyma involved. Hemoperitoneum was detected in 14 patients, and characterized as small in $8(12 \%)$, moderate in three (3.2\%), and large in $13(3.2 \%)$.

Conclusions: CT was useful in establishing the location and extent of injuries, and in detecting the presence of blood or air in the peritoneal cavity. However, the extent of injury to solid viscera detected on CT did not correlate with the need for laparotomy. Of 52 moderate to severe anatomic injuries of the liver, spleen or kidney, only ten (5.2\%) required surgical intervention because of persistent bleeding Laparotomy occurred more frequently in the presence of a large hemoperitoneum, with moderate to large hemoperitoneum required surgical exploration. This analysis confirms the usefulness of CT for detection of location and extent of injury in patient with blunt abdominal trauma presented in our Hospital.

Keyword: laparotomy, abdominal computed tomography, hemoperitoneum

\section{Evaluation on Desarda Technique in Inguinal Hernia Surgery.}

Dritan ÇOBANI ${ }^{1}$, Agron DOGJANI ${ }^{1}$, Etmont CELIKU ${ }^{2}$, Mihal $\mathrm{KERCI}^{3}$

${ }^{1}$ General Surgeon at the University Hospital of Trauma, Tirana, ALBANLA.

${ }^{2}$ Department of Surgery, University Hospital Center "Mother Teresa", Tirana, ALBANIA

${ }^{3}$ University of Medicine of Tirana, ALBANIA.
Abstract

Inguinal hernias are a widespread pathology and every fourth man in his life will be affected by this pathology. Worldwide, more than 20 million patients undergo inguinal hernia repair annually.

Desarda, in 2001 has described a new method of inguinal hernia repair, which does not require prosthetic mesh, it is a tensionFree Repairs without Mesh, in Inguinal Hernia Surgery.

This technique does not require the implantation of a foreign body as a mesh and does not produce major complications during or after surgery.

Material and Method: A descriptive retrospective study was conducted at University Hospital of Trauma in Albania. From April 2016 - March 2018 were recorded all the data for patients who presented to our hospital and underwent inguinal hernia repair in our hospital, under the conditions of elective surgery.

Results: In this period had a total of 142 patients with inguinal hernias were enrolled in the study. The median age of patients was 46,12 years (range 14 to 92 years old).

Males outnumbered females by a ratio of 18.3:1. This gender deference was statistically significant $(\mathrm{P}=0.004)$. Most patients $130(23.9 \%)$ presented late (more than one years of onset of hernia).

Conclusion: Surgical repair of primary inguinal hernia using the Desarda technique is a simple, feasible, repeatable procedure, using the patient's own tissues.

It seems that the Desarda repair can still be a safe alternative to other non-mesh surgical techniques, especially when the patient refuses the use a synthetic mesh. Desarda repair has the potential to become the gold standard of hernia repair.

Keywords: inguinal hernia, Desarda, modified Bassini, Lichtenstein 


\section{Errors in Radiology and Strategy to Reduce them.}

\author{
Altina XHAFERI ${ }^{1 *}$, Medien XHAFERI ${ }^{2}$, Ilir \\ LUMI $^{3}$ \\ ${ }^{1}$ Radiologist, University of Medicine, Tirana, \\ Albania. \\ ${ }^{2}$ Angiosurgeon, University Hospital of Trauma, \\ Tirana, Albania. \\ ${ }^{3}$ Radiologist technologists, University Hospital of \\ Trauma, Tirana, Albania.
}

\section{Abstract \\ Introduction: Diagnostic error in medicine is a major cause of patient harm, with the rate of missed, incorrect, or delayed diagnoses estimated to be as high as $10 \%-$ $15 \%$. Autopsy studies have identified major diagnostic discrepancies in up to $20 \%$ of cases, suggesting that the working or final clinical diagnosis may be wrong in as many as one in five patients overall} Material and Methods; Here is a review of errors in diagnostic radiology, in an attempt to explain possible underlying causes of these errors. The authors also propose strategies for error reduction in radiology. On the basis of current understanding, specific suggestions are offered as to how radiologists can improve their performance in practice.

Results: Radiologic errors have been categorized somewhat differently by various authors. The topic is made more complex in that the definition of what constitutes an error in radiologic interpretation is subject to debate. Surgically or autopsy-proven diagnoses are generally accepted as an objective reference standard, but these rarely apply.

Conclusion: We hope that future research toward understanding the underlying processes of human perception and overcoming the inevitable cognitive biases that humans bring to their tasks will improve the likelihood that radiologist errors in practice can be reduced. Quality improvement strategies and information technology-based solutions may also provide substantial benefits.

Key words: Error, radiology, radiologist, diagnose

\section{Primary Bone Marrow Edema Syndromes}

Krenar PREZA ${ }^{1}$, Almir SKANA ${ }^{2}$,

${ }^{I}$ Chief of the Imaging Department, Continental

Hospital, Tirana. Albania

${ }^{2}$ Radiologist, Continental Hospital, Tirana Albania

\begin{abstract}
Bone marrow edema is a pattern of marrow alteration frequently observed at MRI. It is defined by the presence of an ill-delimited area of moderate and homogeneous decrease in signal intensity on SE T1 images that converts to high signal intensity on fat-saturated proton density or T2-weighted images. It is non-specific and may be associated with almost any abnormal marrow, bone or joint conditions. Epiphyseal bone marrow edema can be associated with self-limited spontaneously resolutive conditions (overuse, stress insufficiency fractures, transient osteoporosis), or with evolutive disorders including chondropathy or spontaneous osteonecrosis. The main task of the radiologist is to assess the cause for bone marrow edema and to highlight imaging features that contribute to a specific diagnosis and subsequently a prognosis (resolutive versus nonresolutive). The current paper aims at emphasizing imaging features indicative of bone marrow edema to avoid confusion with systemic osteonecrosis. We will also highlight imaging features of prognostic significance that enable the clinician to tailor the treatment to the patient's condition.
\end{abstract}

Key words: MRI, bone edema syndrome, avascular necrosis. 


\section{Role of Ultrasound with Color Doppler in Acute Scrotum Management}

Maksim ÇELA $^{1}$, Erton KARAFILI ${ }^{2}$

${ }^{1}$ Freelance Professor at Imaging Tirana, Albania

${ }^{2}$ Erton Clinic, Tirana, Albania

\begin{abstract}
Background: Acute scrotum is a common medical emergency. Doppler Ultrasound (DU) is a non-invasive diagnostic tool with high sensitivity in the triage of acute scrotum. It is the modality of choice to differentiate testicular torsion and traumatic testicular rupture from non emergent conditions. This also applies to B-mode, colour Doppler, spectral Doppler and power Doppler Ultrasound.
\end{abstract}

Aim: This article aims to evaluate the role of ultrasound with color doppler in acute scrotum management

Materials and Methods: In our study we evaluated 13 patients aged 7 to 65 years, presented to our department from year 2014 to 2017. Scrotum was examined by Toshiba machines EK and GE. The machine was equipped with high resolution and colour Doppler linear probe 7-12 MHz Toshiba and 7-12 MHz GE. Colour Doppler and power Doppler was used after B-mode and then spectral Doppler to evaluate the blood flow. Results: Only 4 patients were selected for our study: 1 patient with torsion of testis and 3 patients with different types orchioepididymitis. Other cases were nonemergent conditions. Two of our four patients were treated surgically and two others were treated conservatively.

Conclusions: We therefore conclude that Doppler Ultrasound (DU) is the ideal noninvasive imaging modality in the triage of scrotal emergencies.

Keywords: ultrasound, colour doppler, acute scrotum

\section{Posttraumatic Pleural Empiema and Multidetector CT Findings.}

Eni MEHMETI ${ }^{1}$, Besmir BULKU ${ }^{1}$, Vidi DEMKO ${ }^{2}$ ${ }^{1}$ University Hospital Center "Mother Theresa", Tirana, Albania.

${ }^{2}$ University Hospital of Trauma, Tirana, Albania.

\section{Abstract}

Background: Posttraumatic Pleural Empiema is a significant complication and the main cause for $2-10 \%$ of victims. Multidetector CT Findings is increasingly used. Our study is an analysis focused on the anatomy of pleura, principles behind fluid formation/reabsorption and imaging approach to assessing pleural effusion and PTPE under-CT evacuation.

Material and methods: The study is conducted on eight (8) patients with PTPE at the University Hospital "Shefqet Ndroqi", in Tirana, during the period, January 2015 - June 2018, by using a MDCT of 128 slice - 64 detector - dual source, SIEMENS, German machine. Results and conclusions: The frequency of post-traumatic pleural injuries with presence of Hydrothorax is $75.6 \%$ in total; second after that of Chest wall injuries $(94.2 \%)$. Among the variable forms are reported Hemothorax $-17.4 \%$ and Pneumothorax $-7.3 \%$.

Empyema is rare $-2 \%$. MDCT is the most sensitive, specific, and accurate imaging modality in the assessment of PTPE and management of patients: demonstrates the significant disorder in patients with normal initial radiographs, indicates changing of management in up to $20 \%$ of cases with abnormal initial radiographs, assists several micro-invasive procedures in order to prevents development of empyema, enables early prediction of respiratory compromise and limits the severe invasive interventions.

Keywords: Posttraumatic Pleural Injuries, Empyema, Multidetector CT 


\section{Percutaneous CT-Guided Multisampling Core Needle Biopsy of Thoracic Lesion}

Arjana SINA ${ }^{1}$, J. PROFILI I ${ }^{2}$, A ARAPI ${ }^{3}$

${ }^{1}$ Radiologist, International Turkish Hospital, Tirana, ALBANIA

${ }^{2}$ Anesthesiologist-Intensivist, Onkology Service, University Hospital Center "Mother Theresa", Tirana, ALBANIA

${ }^{3}$ Residency, Anesthesiologist-Intensivist, University Hospital Center "Mother Theresa", Tirana, ALBANIA.

\section{Abstract \\ Objective: The purpose of our study was to evaluate the diagnostic yield and the complication rate of percutaneous CT- guided coaxial 18-gauge $(1.25$-mm diameter) multisampling (five samples) core needle biopsy (CNB) of suspected thoracic lesions.}

Materials and Methods: The records of 350 consecutive patients (100 women, 250 men; age range, 18-80 years) who underwent percutaneous CT-guided adjustable coaxial 18-gauge multisampling (five samples) CNB of a suspected thoracic lesion.

Results: Ninety-seven percent (339/350) of CNB specimens were considered adequate for a specific diagnosis by the histopathology staff. There were 330 malignant lesions and 20 benign lesions. There was no false negative result when CNB was considered adequate for a specific diagnosis by the histopathology staff. Pneumothorax occurred in $8 \%$ (28/350 intrapulmonary lesions). Two patients required placement of a chest tube. Minor postbiopsy hemoptysis occurred and resolved spontaneously in 5\% (17/350) of patients.

Conclusion: Percutaneous CT-guided coaxial multisampling large $\mathrm{CNB}$ of suspected thoracic lesions, in a mainly cancer-based population, is an accurate procedure for a specific histologic diagnosis and has a low rate of complications.

Key words: Biopsy, CNB, Needle.

\section{Imaging Characteristics and Prevalence of Pancreatic Carcinoma in Kosovo During 2011-2015}

Kreshnike DEDUSHI HOTI ${ }^{1}$, Serbeze KABASHI MUCAJ $^{1}$, Astrit HOXHAJ ${ }^{1}$

${ }^{I}$ Radiology Department University Clinical Center of Kosovo, Pristine, Kosovo.

\section{Abstract \\ Background: Pancreatic cancer is the $10^{\text {th }}$ most common malignancy and the $4^{\text {th }}$ largest cancer killer in adults.}

The aim of this paper is to evaluate the number of cases presented with pancreatic carcinoma during the years 2011-2015, our experience of the imaging characteristics of pancreatic carcinoma. We evaluated prevalence of the pancreatic cancers, distant metastases and other local infiltration signs among the total cases of the pancreatic cancers diagnosed in the University Clinical Center of Kosovo, with the aim to compare these research findings to similar studies made in the developed countries.

Material and Methods: This is a retrospective research study done during the period of 2011-2015. This retrospective research study includes 362 patients recently diagnosed with pancreatic cancer, examined in the period of 2011-2015 at the University Clinical Center of Kosovo. The imaging diagnostics are performed with MSCT Sensation 64 Siemens, MSCT Emotion 6 Siemens, and 1.5T MRI Symphony Siemens, biopsy guide with MSCT Sensation 64 Siemens in the Radiologic Clinic of UCCK; HP has been performed in Clinic of Pathology at UCCK and prevalence is taken from the number of cases Reported at the Institute of Oncology Institute of Statistics and NIPH.

Results: Out of a total of the 362 patients diagnosed with pancreas cancer, results is female $39.5 \%(n=143)$ and male $61.5 \%$ $(n=219)$, report M: F (1: 1.6), 286 cases resulted in head and neck $79 \%(n=286)$, 76 cases resulted in body and tail cancers 
(21\%), distant metastases in first imaging modality were found in $(n=155)$.

Conclusion: The prevalence is 19.9: 100,000 inhabitants. Prevalence of carcinoma of the pancreas for 5 years in Kosovo has proved to be prevalence is 19.9: 100,000 inhabitants. Seventy-four percent $(74 \%, n=268)$ of all cancers are found in Stage III and IV. From an imaging point of view, these cancers were presented in an advanced stage, mainly due to their late clinical symptoms and limited access to imaging methods in our country. Keywords: Pancreatic Cancer, MSCT, MRI, Prevalence, UCCK Pristina, Kosovo

\section{Role of Radiology in the Management of Traumatic Injuries to the Abdominal Solid Organs - with a Focus on Spleen}

Sonja (Saraçi) BUTORAC ${ }^{1}$, Vidi DEMKO ${ }^{1}$, Admir MUSTAFA $^{1}$, Kujtim ALIMERI ${ }^{1}$, Gezim GURI ${ }^{1}$, Artan GJIKA ${ }^{1}$

${ }^{1}$ Department of Radiology, University Hospital of trauma, Tirana, Albania.

\footnotetext{
Abstract

Background: The morbidity, mortality and economic costs resulting from trauma in general, and blunt abdominal trauma in particular, are substantial. In the past, many traumatic abdominal injuries had to be assessed and treated surgically, and were therefore associated with the additional risks and complications of surgery. Increasing availability and developments in CT technology, such as multi-detector CT (MDCT), have enabled rapid nonsurgical assessment of trauma patients in the emergency room, thereby significantly reducing the number of exploratory operations.

Imaging Findings and Procedure Details: In our practice, US (FAST) has constituted the initial survey of examination for most patients, unless patients showed new signs of declining hemodinamically, in which case they underwent MDCT urgently, especially as MDCT is characterized by
}

faster scanning times, increased volume coverage, and improved spatial and temporal resolution. However, the initial survey with US was followed by a more thorough examination with MDCT, in most cases contrast-enhanced, which in our practice constitutes the imaging modality of choice in the initial emergency evaluation and triage to different management strategies in abdominal solid organ trauma-the spleen, liver and kidney.

Conclusions: Imaging findings, in addition to the patients' clinical and haemodynamic status, have played a crucial role in the evaluation and management of trauma patients. CT has a vital role in assessing the extent of injury to intra-abdominal organs and assigning an injury grade, which may affect further the management. While, CT has virtually replaced abdominal surgical exploration for the detection of important injuries; nonsurgical treatment has become the standard of care in hemodynamically stable patients with blunt trauma to solid abdominal organs such as the spleen, liver and kidneys.

Key Words: Imaging Findings, blunt abdominal trauma, solid organ injuries

\section{Transport of Injured Patient. A Nursing Approach.}

Daniela BIMI ${ }^{1}$, Indrit BIMI ${ }^{1}$

'University "Aleksander Moisiu”, Durres, Albania 
tasked with the transport of increasingly severe injuries.

Guidelines for transport within the hospital (intrahospital) and between hospitals (interhospital) have been formally developed by the American Society of Anesthesiologists, the American College of Surgeons Committee on Trauma, and the Society of Critical Care Medicine, among others.

Three common themes emerge with all of these guidelines: (i) patient transport always involves some degree of risk, (ii) the benefits to be realized must outweigh these potential risks, and (iii) during transport, the standards of care (especially monitoring) should be at the same level or higher than that provided in the setting from which the patient is being transported.

The level of care during transport should be equal to or exceed the level of care the patient is receiving prior to departure.

Keywords: trauma, injured patient, transport

\section{Postoperative Pain Management, the Role of Surgical Ward Nurses.}

\author{
Aferdita ADEMI ${ }^{1,2}$, Ardita OSMANI ${ }^{1,2}$, Blerim \\ FEJZULI ${ }^{1,2}$ \\ ${ }^{1}$ Clinical Hospital - Tetovo, Macedonia \\ ${ }^{2}$ Medical Faculty - University of Tetovo, \\ Macedonia
}

\begin{abstract}
Introduction; Postoperative pain is the most undesired consequence after surgical intervention, and if not managed properly may delay postoperative recovery.

Objective: To estimate the incidence and intensity of pain as well as the mode of treatment and the role of nurses in management of postoperative pain.

Material and Methods: A total of 365 patients were interviewed in post-operative period for pain intensity after surgical intervention. We used the Numerical Rating Scale (0-10) for evaluation of pain intensity.
\end{abstract}

Results: from 365 patients included in this study 195 of them were males and 170 females with an average age of 47.8 years. The incidence of pain in a postoperative period was $95 \%$, whereas the average level of pain intensity at the time of worst pain according to Numerical Rating Scale was approximately 7.1. Treatment of postoperative pain is accomplished through NSAIDs, opioids drugs, or combinations of them. Monitoring by nurse of patient response to postoperative pain is of great value and very important in pain management.

Conclusion: The nurses played an important role in the management and treatment of postoperative pain.

Key words: nurses, postoperative pain, management

\section{Colles' Fracture and Rehabilitation}

Anxhela AHMATAJ ${ }^{1}$, Zamir DEMIRAJ ${ }^{1}$

${ }^{1}$ Physical Therapist at University Hospital of Trauma, Tirana, Albania

${ }^{1}$ Orthopedic service at University Hospital of Trauma, Tirana, Albania

\begin{abstract}
Colles' fracture, the fracture of distal radius, accounts for a high incidence of fractures in Orthopedics. These fractures occur mostly in post menopausal women, due to osteoporosis. In this group, this kind of fracture follows after a minor trauma. These fractures rarely happen in young generation; they require a high energy trauma, such as car accidents.

Treatments of them varies from conservative to surgical treatment (ORIF, fixative extern, etc.).

Complications are seen in most of the cases. Physiotherapy is an important procedure to get optimal clinical results, after conservative and surgical treatment. The time to start physical therapy is recommended since the first post surgery days. Passive ROM of digital articulation is started to improve ROM and muscles' strength.
\end{abstract}


After ORIF treatment it continues with

Radio-Carpal movements and prono supinator movements.

For complicated cases, such as algodistrophy, physical therapy takes an important role in the treatment of complications, particularly the MLD (manual lymphatic drainage).

Based on our experience and according to evidences in such scientific literatures, it's seen a better improvement, measured with VAS analogue scale, DASH, ROM, and return to ADL (activity daily life) in the patients that have been subject of a rigorous physical therapy protocol, compared to those who haven't followed physiotherapy for different reasons.

Keywords: physical therapy, rehabilitation, Colles fracture

\section{Nurse Follow-up in Cast Immobilised Patients.}

Arben GJONEJ ${ }^{1}$, Risida GJONEJ ${ }^{2}$, Astrit PEKA ${ }^{1}$

${ }^{1}$ Orthopedic service at University Hospital of

Trauma, Tirana, Albania.

${ }^{2}$ Lecturer at Faculty of Technical Science, Medical University of Tirana, Albania.

\section{Abstract}

Background: Cast is a material used for immobilisation of fractures and for treatment of born and gained orthopaedic pathologies. It is applied from the orthopaedic doctor and cast technician. Earlier these procedures were applied by non medical professions.

Aim: This study aims to: educate the patient and his relatives, improve cast immobilisation by following certain techniques, avoid further damage and later complications, etc

Materials and Methods: This is a descriptive study which evaluates the whole cast immobilisation process, and in each moment shows the key points which have to be considered. There are three reasons for cast immobilisation; fractures immobilisation, treatment of orthopaedic pathologies and support after an surgical orthopaedic procedure.

Results: There are three reasons for cast immobilisation; fractures immobilisation, treatment of orthopaedic pathologies and support after an surgical orthopaedic procedure. There are also three types of casts; classic cast, plastic cast and bandage cast.

Conclusions: Cast immobilisation is a non invasive procedure which if applied correctly offers very good results for fractures. Each case should be evaluated promptly because cast immobilisation has its limitations. Taking into consideration the cost - effectiveness ratio, we recommend the cast immobilisation to be the first choice in orthopaedic treatment. Keywords: cast, orthopaedic procedure, fractures immobilisation

\section{ALARA; Reducing Radiation Risks from Computed Tomography Scanning in Children.}

Jona ISAKU ${ }^{1}$, A.SINAJ ${ }^{2}, Q$.ARAPI 3

${ }^{1}$ Radiologic technician, International Turkish

Hospital Tirana, Albania.

${ }^{2}$ Radiologist, International Turkish Hospital Tirana, Albania.

3Resident Radiologist, Mother Teresa Hospital Center, Tirana, Albania.

\begin{abstract}
Introduction: Radiation exposure from computed tomography is associated with a small but significant increase in risk for fatal cancer over a child's lifetime. This article aims to highlight awareness and spearhead efforts to reduce unnecessary computed tomography scans in children. Materials and methods: In our study 7200 cases are registered that have done a CT in QSUT for the ages 0 to 14 years old. In our study population we have taken 42 cases, in ages between $0-12$ years old, residents in Tirana country. Pediatrics recommend to do a CT based on the principles of ALARA only if it is necessary to help in the diagnostcation of the case .Technical
\end{abstract}


staff at Pediatric Hospital applies the reduced dose radiation system for pediatric ages. The CT machine in this hospital is made up of a small number of detectors affecting the quality of the image. As a result, $36 \%$ of the cases have repeated the examination. In our patients the radiation dose in $74 \%$ of them ( 31 cases) is between $0-0.5 \mathrm{~Gy}$, in $12 \%$ the dosage catches the levels between $0.5-1.8 \mathrm{~Gy}$. In these patients in long terms we have diagnosed slight hemapoetic changes. In $8 \%$ of cases the patients have taken a total dosage exposure between 2-3.7 Gy.

Conclusions: The effective dose allowed for the population in one year is $1 \mathrm{~m} \mathrm{~Sv}$, in our study the average dose taken by the population of study is $2.35 \mathrm{~Gy}$. This value is legitimate within the effective permissible dose. Ongoing education of technician personal during the last year has been increased by $30 \%$ with the purpose of minimizing the errors during the $\mathrm{CT}$ examination

To summarize, health policies and clinicians doctors should stimulate the implementation of alternative diagnostic techniques such as Ultrasound and MRI. Key words: ALARA, diagnose, CT, radiation, risks, effective dose.

\section{Clavicle Fractures: How to Avoid Complications from the Cast Technician}

Arben GJONEJ ${ }^{1}$, Risida GJONEJ ${ }^{2}$, Astrit PEKA ${ }^{1}$, Edvin SELMANI ${ }^{1}$

${ }^{1}$ Orthopedic service at University Hospital of Trauma, Tirana, Albania.

${ }^{2}$ Lecturer at Faculty of Technical Science, Medical University of Tirana, Albania.

\begin{abstract}
Background: Clavicle fractures are very frequent in daily practice. They affect mostly the middle third and mainly male patients. Factors that contribute are different for different group ages.
\end{abstract}

The Aim: The aim of this study is to evaluate the care and how to avoid complications form the cast technician prospective in treatment of clavicle fractures during 2014-2015 in our Service and to find its incidence during this period. Material and method: We collected data form the cast registry of Orthopedic and Trauma Service in the University Trauma Hospital during period January 2014December 2015. In our study we excluded cases that were treated surgically.

Statistical analysis of the data was done with SPSS version 2.0 packages.

Frequencies and percentages are used to analyze the categorical variables. $\mathrm{P}$ value < 0.05 was considered significant.

Results: 150 cases were treated with clavicle fracture. They were $5(3.3 \%)$ newborns. Age 1-5 years old male patients were $13(8.7 \%)$, in the group age 6-14 years older there were $8(5.3 \%)$ and male patients of the group age 15-25 years old there were $54(36 \%)$. Male patients of group age 26-50 years old there were 30 $(20 \%)$ and more than 50 years old there were 10 patients $(6.7 \%)$. From our study the female patients of 1-5 years old there were $7(4.7 \%), 6-14$ years were $7(4.7 \%)$, $15-25$ years old were $7(4.7 \%), 26-50$ years old and more the 50 years old were 5 (3.3\%). In total there were 115 male and 35 females patients. In 115 male patients $80(69.6 \%)$ were right side and $35(23.3 \%)$ were left side. In female group 35 (23.3\%) 20 were right side and 15 were left side. The management shows a significant correlation between the diagnosis and the type of immobilization with $\mathrm{p}<0.05$. Conclusions: Clavicle fractures during 2015-2016 shows a ration of 4.3/1 male / female the same as international literature. Treatment according to age groups is achieved with leucoplast immobilization or cast or braces. Treatment was successful without complications.

Keyword: Clavicle, fracture, risk factors, cast 
127. The Effect of the Nurses' Knowledge and Practices in the Peripheral Intravenous Catheterization and in The Development of Infections/Complications Related with Catheterization.

Arbëresha GURGURI ${ }^{*}$, Fatjona KAMBERI ${ }^{2}$

${ }^{1}$ Public Health Faculty, "Ismail Qemali" University, Vlora Albania

${ }^{2}$ Public Health Study Center, Public Health Faculty, "Ismail Qemali" University, Vlora Albania

\begin{abstract}

\section{Introduction}

Peripheral intravenous catheterization is one of the most common invasive procedure to provide access of the administration of drugs ,fluids and parenteral nutrition in hospitalized patients. This procedure is associated with a high relatively risk for complications, with serious consequences in patients' health and increase the costs of healthcare.Aimed at assessing the impact of knowledge and practices of staff nurses on peripheral IV catheterization and its complications in the Department of Pathology and Surgery of Vlora Hospital.
\end{abstract}

\section{Methods}

In the analytical observational study ,54 randomly selected nurses who completed an anonymus questionnaire for the IV peripheral catheterization and related complications, and 14 of them were observed during the course of the procedure in 30 hospitalized patients during the period of conducting the study .The statistical analysis included the calculation of averages ,frequencies and percentages while the Chi-Square test was used for the relationship between the variables .

\section{Results}

The data collected showed that the majority of nurses $(67 \%)$ had average knowledge and average practice $(83 \%)$ about peripheral IV catheterization and the related complications. There was a significant relationship between the gender of the nurses and the level of knowledge $(\mathrm{p}=0.0236)$ while there have been observed some important relationships between specific questions with demographic variables of nurses. Regarding nursing practice, there were significant relationships between the number of complications with the number of attempts and the appearance of the edema, output of the catheter with the number of attempts, respectively ( $\mathrm{p}=0.005$ and $\mathrm{p}=0.003$ ). Complications were observed in $68.97 \%$ of the insertions, with

erythema,pain ,edema,fever being the commonest $(23.81 \%)$.

\section{Conclusions}

Nurse's knowledge about peripheral IV catheterization procedure affects the occurrence of complications related to the technique.Increasing knowledge and focusing on specific measures that are overlooked in practice are essential to improving the procedure with a direct impact on reducing complications, cost of care and enhancing health care quality. Key words: Knowledge, practices, peripheral intravenous catheterization, nurse,complications, patient .

\section{Obstetric Trauma and Midwifery.}

Daniela BIMI ${ }^{1}$, Indrit BIMI ${ }^{1}$

${ }^{1}$ University “Aleksander Moisiu”, Durres, Albania

\begin{abstract}
Trauma is the most common nonobstetrical cause of maternal and fetal mortality affecting $6 \%$ to $7 \%$ of pregnancies. Trauma care of the parturient involves evaluation and resuscitation of two individuals (mother and fetus) and occasionally perimortem cesarean section to promote fetal survival despite mortal wounding of the mother. The primary principle in management of the traumatized parturient is prompt and sustained resuscitation of the mother, thereby providing the best opportunity for
\end{abstract}


a favorable outcome for both the mother and the fetus.

Management of the pregnant trauma patient is challenging because pregnancy imposes physiologic alterations that complicate both evaluation and resuscitation.

Additionally, advanced pregnancy may influence the pattern of injuries sustained due to uterine displacement of intraabdominal structures. This paper reviews the important information that must be understood by all members of the trauma team to assure the best possible outcome. Keywords: trauma, parturient, fetal mortality, resuscitation

\section{FAST Ultrasound Method for Trauma}

Dorian META $^{1}$

${ }^{I}$ Faculty of Technical Sciences, University of Medicine Tirana

\begin{abstract}
Background: One of the main problems regarding trauma (especially the abdominal ones and not only) is the haemorrhage, which may be early diagnosed by the FAST method and a well-trained nursing staff. An ultrasound made in real time can be helpful to determine the diagnosis. Diagnosis and treatment are essential for optimum results and maintaining the integrity and patient's life
\end{abstract}

Aim: The early identification of abdominal traumas and the real-time determination of the traumatic event and its consequence comprise an increasing challenge, both real and indispensable. The first line of life is precisely the efficient and professional response to trauma.

Results: There has been an increase in the post trauma survival index and even during the hospitalization phase in $60 \%$ of patients who have received treatment service with the use of FAST. On the other hand, in patients, who were not treated with the FAST method, there has been observed a drastic decline of $40 \%$ in the percentage of trauma survival and complications were encountered while giving first aid.

Conclusions: The nurse is required to have some specific ultrasound knowledge in order to be part of the FAST program, as the appropriate diagnosis and the high level of knowledge has a direct effect on the patient's life. They should be trained and capable of managing hemodynamic problems, intensive care techniques and drug administrations, as well as manipulations such as, intubation or pericardiocentesis.

Keywords: trauma, FAST, nurse, early diagnosis

\section{Radiology Techniques in Specific Pathologies}

Ilir LUMI ${ }^{1}$, Erjona ZOGAJ ${ }^{1}$

Msc Radiologist Technician in Trauma University Hospital of Tirana, ALBANIA

\section{Abstract \\ Introduction: Radiology is a health} science developed more lately according to other health sciences. In 1895 Rontgen discovered the X-ray that opened the door to other new examination for patients. Even though, despite this fact, today we have great technology that brings to us $100 \%$ qualified images.

A variety of imaging techniques such as $\mathrm{X}$ ray radiography, ultrasound, computed tomography (CT), nuclear medicine including positron emission tomography (PET), and magnetic resonance imaging (MRI) are used to diagnose and/or treat diseases. . We have a lot of radiology techniques and also specific one for different pathologies. Knowing well the pathologies and those specific techniques brings the Radiologist Technicians in a different level and also to a better radiology service. Having a good collaboration between staff radiologist physician is a very important part of the work in radiology department.

Purpouse: It is important to have information and to be trained for different 
techniques that improves the quality of images and also to have a good collaboration with physician to reduce the possibility of redoing the examination again or leading the doctors in a wrong diagnosis.

Material and Methodology: This study is a Descriptive research. The database is taken in Trauma University Hospital of Tirana. We have selected 10 specific pathologies that requires specific technique to be more noticeable in medical image. Comparing the wrong images with the right ones is the main format of this study, opened for discussion.

Conclusion: Informing and organizing more trainings or conferences to discussed different techniques is very important. The importance of the collaboration between the staff for a better medical service should be emphasized in every conference.

Key Words: Pathology, radiology, Patient, communication, ALARA

\section{Amputation Versus Salvage in Open Tibial Fractures Grade III}

Jordan SAVESKI ${ }^{1}$, Neda TRAJKOVSKA ${ }^{1}$, Emilija STOJKOVSKA-PEMOVSKA ${ }^{2}$.

${ }^{I}$ Clinical Hospital Acibadem, Sistina, Skopje, MACEDONIA

${ }^{2}$ University Clinic of Traumatology, Medical Faculty, Skopje, MACEDONIA

\section{Abstract}

Introduction: Mangled extremity injury (MEI) are result of high energy trauma and are so severe that amputation is possible outcome. The first decision that must be made by the surgeon is: whether salvage is possible at all or not salvage is advisable.

Purpose: The purpose of this presentation is how decision is made about salvage or amputation.

Material and methods: From 1997 to 2017 year 360 open tibial fractures were surgically treated. Of them, grade III was 120 - grade IIIA 50, grade IIIB 46 and grade IIIC 24. Amputation was done in 18 patients, 8 primary and 10 secondary.
Scoring systems defining objective criteria for amputation and salvage was discussed. The goal was to assist the surgeon in making the initial decision. The final goals are stable, painless limb and intact sensation. Indications for primary amputation: complete traumatic amputation, severe crush injuries with warm ischemia time $>6 \mathrm{~h}$, irreparable sciatic/tibial nerve injury, long segment or multilevel arterial injury and associated life-threatening injuries. Secondary amputation is related with higher morbidity, higher mortality and higher hospital costs. Recently the Ganga Hospital Score and the protocol of WTA Western Trauma Association provide more objective criteria to make decision whether to perform salvage or amputation.

Conclusion: Beside the points, sums, calculations, when it is not a situation LIFE OVER LIMB, .when in doubt, even minimal.....consult a colleague, discuss with the patient if possible, face the family with the facts... at least be absolutely sure that you are doing no harm. Amputation or salvage is and will be one of the hardest decisions that a trauma surgeon should make.

\section{Knee Sanguine Effusions in Sports Trauma (Haemarthrosis)}

Ilia MAZNIKU ${ }^{1}$

${ }^{I}$ Faculty of Technical Medical Sciences, University of Elbasan "A. Xhuvani", Elbasan, Albania

\begin{abstract}
Background: The blood collection in the athlete's knee without suffering injuries of the soft tissues represents a dangerous potential to the future of the athlete.

Aim: In the following material based on retrospective data we aim to represent the cases of competitive athletes who suffered traumatic knee effusions.
\end{abstract}

Materials and methods: This is a retrospective study during the 1980-2015time frame. The patients inculded in the study were competitive athletes with 
regular activities and training involving a timeframe from 3 to 12 years.

Results: In overall 16 patients were included into the study (13 men and 3 women). The average age of the injured athletes was 23.4 years, the youngest of them 14 years whereas the eldest 28.7 years. The right knee was injured on 12 cases $(75 \%)$, whereas the left knee on 4 cases $(25 \%) .7(44 \%)$ cases were basketball players, 4 cases $(25 \%)$ volleyball, 3 cases (19\%) athletics 1 case $(6 \%)$ and wrestling 1 case $(6 \%)$. Surgery was performed in 6 athletes (37.5\%).

Conclusions: Traumatic haemarthrosis of the knee articulation at the sportsmen and women is only the outer side of the injury. It has consequences quite severe with the future of the athlete. An in-depth study of the accompanying injuries is a primary duty of the sports team medical doctor, and a request filled with hope by the sportsmen to have their problem solved. The correction of the accompanying injuries, with or without invasive surgery is a necessity to the successful continuation of the sports activity.

Keywords: Haemarthrosis, Sports Trauma

\section{The Masquelet Technique on the Treatment of Fractures with Segmental Bone Loss. A Case Report.}

Zamir DEMIRAJ ${ }^{1}$,

${ }^{1}$ Orthopedic service, University Hospital of

Trauma, Tirana, Albania

\begin{abstract}
Background: Fractures of the diaphyseal bones with major bone defects are injured not very frequently in clinical practice. They usually occur after a trauma with high energy (like car accident) and are accompanied by other injuries like the trauma of the head and the trauma of the thoraco-abdominal region, and also other fractures. These fractures are accompanied with significant long-term morbidity, this because their treatment has high
\end{abstract}

difficulties when talking about the synthesis or the consolidation. We should also add here the fact that most of them are open. This is the case that we will present in our study: Open fracture of the supercondylare-diaphyseal femuri left, type III-A fractures according to GustiloAnderson. There are some ways for their treatment.

The Masquelet technique was presented by the French surgeon Masquelet. The interference is done in two phases, the first stage is the filling of the defect in the bone with cement and for a period of 2-4 months, around the cement an iterative membrane (Masquelet's membrane) is created; during the second stage we take off the cement spacer and fill the cavity with bone grafting (autolog transplant) while naturally protecting the created membrane. The membrane acts as a bioreactor for consolidation. This technique we have used on the case we are about to present.

Patient M.D. 35 years old, after a car accident is presented at the Trauma Hospital in a state of traumatichemorrhagic shock with the head trauma, thoraco-abdominal trauma and the left mandibular fractures, the left forearm fractures, diaphysis fracture of the left femur, with the segmental bone loss about $8 \mathrm{~cm}$, open type III-A according to Gustilo-Anderson.

Keywords: Masquelet; fracture; segmental bone; cement; DCS dynamic condylar screw; 


\section{The influence of Damage Control Orthopedic Surgery (DOC) in the Polytrauma Patients}

Blerta HAMOLLI ${ }^{1 *}$, Arnel MICI ${ }^{1}$, Marjola QATIPI $^{2}$

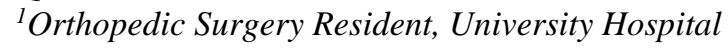
of Trauma, Tirana, ALBANIA

${ }^{2}$ Student of University of Medicine, Tirana, ALBANIA

\begin{abstract}
Background:Damage control orthopedics is a relatively new concept and it occupies a small proportion of the overall process in a polytraumatic patient. It necessitates continuous communication between surgical, anaesthetic and the whole multidisciplinary team. The only randomized study showed a benefit for borderline patients treated by DCO in comparison to early total care. The damage control strategy has been adopted by traumatologists to treat extremity fractures. Purpose: The aim of the study is to evaluate the concept of damage control by immediate external fracture fixation (DCO) and consecutive conversion osteosynthesis in terms of time saving and safety.
\end{abstract}

Patients and Methods: For the study we considered 33 patients (19 Males and 14 Females) from 21 to 50 years old with severe polytraumatic damages, including even orthopedic injuries that were managed with DCO during the period of 6 months (from January 2018 to June 2018) in University Hospital of Trauma, Tirana. 9 of 33 patients were send to the ICU. The other patients were subject of surgery in terms of

DCO, that consisted on:

a) Immediate life-saving surgery, which was required in 21 cases;

b) Surgery for controlling heavy bleeding, which was required in 7 cases;

c) Wound bleeding control, infection control, washing, dressing, and temporally closing the wound; and

d) Provisional minimally-invasive external fixation, which was performed in 5 patients.
Results: The study demonstrated the enormous time savings and reduction of blood loss during initial treatment if patients with multiple injuries are treated according to DCO. We feel that DCO is a safe strategy in severely injured patients with multiple injuries who are too critically ill for definitive surgery and for those patients who cannot be safely assigned to the clinical pathway of early total care.

Conclusion: The method was adapted to gain control of an unstable situation until definitive intervention could be undertaken

Keywords: polytrauma, wounds, external fixation

\section{Open Fractures of the Tibial Diaphysis External Fixator as a Primary Treating Technique versus Other Treatment Choices}

Ilir HASMUCA ${ }^{1}$

${ }^{l}$ Orthopedic Service University Hospital of Trauma, Tirana, Albania

Abstract
Open fractures of the tibia are more
common than in any other long bone. Rate
of tibial diaphysis fractures reports vary
from 2 per 1000 population to 2 per 10,000
and of these approximately one fourth are
open tibia fractures. Motor vehicle
accidents, skiing accidents, and high-
energy falls are the common causes of
open tibial fractures. The mechanism of
injury determines the fracture
configuration (eg, skiing injuries typically
cause spiral fractures). After primary
debridement, the surgical setup should be
changed and the limb redraped without
losing sterility. Today, the indication in the
worldwide literature for treating exposed
tibial shaft fractures, of Gustilo and
Anderson grades I and II, III consists of
immediate fixation using an intramedullary
nail. External fixation is used for types
IIIA and IIIB fractures. The technical
resources or implants for immediate


treatment of these exposed fractures are not always available. Thus, temporary external fixation is the option in such cases. Monolateral external fixators generally are preferred for the tibia, though multiplanar and circular fixators provide greater stability.

The purpose of this retrospective study was to evaluate the results and effectiveness of external fixator as a primary and definitive treatment in open fractures of the tibial diaphysis and compare it with other modalities of treatment.

Keywords: Open fractures, tibial diaphysis, external fixator

\section{Open Fractures of Tibia and External Fixationin Injured Patients. A Case Report}

Enkelei BALLA $^{1}$

${ }^{1}$ Orthopedic and Trauma Service, University Hospital of Trauma, Tirana, Albania

\section{Abstract \\ Background:The tibia is a subcutaneous bone and tibias fractures are frequently open fractures. An open fracture is a fracture in which there is an open wound or break in the skin near the site of the broken bone.}

Aim: To provide management of open fracture of tibia

Methodology: The study was conducted in the University Hospital Center of Trauma, Tirana, during the period of time of January to August 2018.

Case study: A 51 years- old male slippedwhere is workingand presents with high energy trauma, an open tibia fracture with significant soft tissue swelling. Early treatment for this wasfocused on stopping hemorrhage, preventing infection at the site of the injury and the surgery was performed within $24 \mathrm{~h}$ after.External fixation was placed for initial as a safe and versatile technique. We provided skeletal stability, access to the site of injury and allowed early mobilization.He returned for follow-up visits at regular intervals and after 6 months he returns to pre-injury activities.

Conclusions:Management of open fractures of tibia include the saving the life of the patient and the limb, to uniting the fracture, to preventing infections and restrationing of functions. External fixation is a safe and versatile technique.

Key words: External fixation, open fractures.

\section{12 Years Albanian Experience with Emergent Surgical Treatment due to Acute Aortic Dissection Type A.}

\author{
Edvin PRIFTI ${ }^{1}$ \\ ${ }^{1}$ Division of Cardiac Surgery, University Hospital \\ Center "Mother Theresa" Tirana, Albania
}

\begin{abstract}
Background:The acute aortic dissection type $\mathrm{A}$ is a critical condition requiring prompt and emergent surgical treatment. The surgical outcomes are associated with high mortality and postoperative morbidity, due to long operation period, bleeding, neurologic complications etc. The aim of this study was to investigate the early and midterm outcome in patients undergoing surgery due to aortic dissection representing the single team experience and to analyze the predictors for poor early and midterm outcome respectively to the type of employed surgical technique and circulatory arrest.
\end{abstract}

Materials and Methods: This is a retrospective study including, 100 patients with acute aortic dissection of the ascending aorta, that underwent emergent surgery during 2006-2018. Mean followup was $4 \pm 2$ years.

Results: Mean age was $53 \pm 18$ years varying between 34 and 79 years old. $61(61 \%)$ patients were males. The extracorporeal circulation time was $178 \pm 56$ minutes and aortic cross clamping time was $38 \pm 41$ minutes. The mean circulatory arrest time was $31 \pm 12$ minutes. The mean lowest central 
temperature was $23.5 \pm 1.1^{\circ} \mathrm{C}$. The mean ICU stay was $5 \pm 7$ day. The hospital mortality was $16(16 \%)$ patients. The cause of death was massive bleeding in 5, cardiac problems in 2, neurologic in 4, MOF in 3, infection in 1, ARDS in 1.16 patienst underwent reopening due to bleeding. Permanent neurologic deficit was identified in 2 survived patients and transitory in 10 patients. Continuous hemofiltration was employed in 16 patients due to acute renal failure. One patients underwent surgery due to endocarditis 1 year later. Two other patients died due to cerebral bleeding at 1 and 0.8 years after surgery. Another patient had a sudden death at 2 years after surgery. The actuarial survival at 3 years after surgery was $74 \%$. The multivariate analysis reavled the preoperative renal failure $(\mathrm{p}=0.03)$, arch surgery $(p<0.001)$, age $>70$ years $(p=0.04)$, and extension of the dissection at the epiaortic vessels $(\mathrm{p}=0.02)$ as strong predictors for overall mortality.

Conclusion: This initial series of patients with acute aortic dissection type A undergoing emergent surgical correction demonstrate acceptable outcome. The hospital mortality is comparable with the eruopean registry. The predictors are similar to other studies. The multimodal treatment is required. Such an extreme presentation can be successfully treated in Albania.

Keywords: aortics disection, type A, acute, Albania, Cardiosurgery

\section{Scoring Systems in Blunt Chest Trauma}

Ayman EL-MENYAR ${ }^{1}$

${ }^{1}$ Director of clinical Research in Trauma and vascular surgery in Hamad General Hospital, Doha, Qatar

\section{Abstract \\ Blunt chest trauma (BCT) represents a significant burden of morbidity and mortality. We aimed to evaluate the scoring tools (Chest AIS, ISS, GCS, TRISS, and RTS) in BCT patients. We}

conducted a retrospective analysis of BCT patients hospitalized between 2011 and 2015. Patients with GCS $\leq 8$ or penetrating chest trauma were excluded.

Of the total of 1928 BCT patients admitted to the level 1 trauma center; 1373 fulfilled the inclusion criteria. The mean age of patients was $34.9 \pm 15.6$ and $90 \%$ were males. There were 229 intubated patients, 197 patients had chest tubes and 30 patients died (2.0\%). ICU and hospital length of stay were significantly associated with polytrauma, RTS, TRISS and positive serum troponin. Age-adjusted predictors of intubation were serum troponin (OR 3.0), RTS (OR 2.1), ISS (OR 1.10) and GCS(OR0.64). Age adjusted predictors of mortality were TRISS (OR 16), serum troponin (OR 9.3), and ISS (OR 1.2). With respect to different thoracic trauma scores, TRISS and serum troponin are found to be appropriate tools for prediction of inhospital outcomes in patients with BCT. There is still extensive work needed to set early, simple scoring tool including anatomical, physiological and biomarker in one set.

\section{Innovations in Cardiac Surgery, Albanian Contribution.}

\section{Edvin PRIFTI ${ }^{1}$ \\ ${ }^{I}$ Division of Cardiac Surgery, University Hospital Center "Mother Theresa" Tirana, Albania}

\begin{abstract}
New surgical techniques have been recently developed by our team in Albania. Some of them have been already accepted in the international surgical armamentarium, some others are in the evaluation process.

1. We developed the "peacock tail" technique is a modification of the cone reconstruction technique for Ebstein anomaly and failing right ventricle, simultaneously to bidirectional cavopulmonary shunt.

2. The "extroverted cuff" technique that we have proposed is a novel modification of the flanged
\end{abstract}


technique for the elephant trunk construction. Such a modification seems suitable for aortic arch aneurysm with ragged descending thoracic aorta that minimizes bleeding from the distal anastomosis and potentially prevents distal embolization of atheromatous plaque.

3. The acute ascending aortic dissection after previous cardiac surgery, in the presence of large aortic dimensions very closed to the sternum, severe aortic regurgitation, false lumen in the descending aorta is a very challenging situation to manage. Herein we are reporting a combination of strategies of perfusion and myocardial protection based on the Port-Access technology that allows antegrade flow into the aorta, endo-clamping of the ascending aorta, administration of cardioplegia before opening the sternum and left ventricular venting to not allow ventricular distention.

4. Tirana 1 technique for total root replacement consist in the same principal of Bentall operation but without coronary ostium detachment and applying a single anastomosis with the valve tube.

5. Tirana 2 technique represents a modified David operation consisting in a external tube wrapping of the aortic root including the coronary ostiums and internal implantation of the valve in a helicoidal form.

6. Retrograde cellular cardiomyoplasty through the coronary sinus according to an experimental study on swines in collaboration with the University of Florence, Italy.Our study provides data in support of the following issues: the venous retrograde delivery route through the coronary sinus is safe and effective;
Celullary cardiomyoplasty provides a significant improvement in function and viability.

Keywords: peacock tail, extroverted cuff, Tirana 1, Tirana 2, Retrograde cellular cardiomyoplasty, inovations, cardiosurgery, Albania

\section{Management of Blunt Thoracic Trauma in Children.}

Emir Q. HAXHIJA ${ }^{1}$

${ }^{1}$ Department of Pediatric and Adolescent Surgery, University Hospital Graz, Graz, Austria
Abstract;
Introduction: Chest trauma in children is an indicator of injury severity and is associated with a high mortality rate. Pulmonary contusions with diffuse pulmonary bleeding, oedema and atelectasis are the most frequent thoracic injuries found. The outcome of pediatric patients after bluntpulmonary contusion is presented.

Methods:The patient population, the injury types, the injury mechanisms, the injury severity score (ISS), the clinical course of chest injuries, and the importance of associated injuries in children less than 19 years of age who were treatedfor pulmonary contusions from 1986 to 2011 have been analyzed. The data from the time 1986-2000have been compared to the dataset 2001-2011.

Results: We found a substantial increase in the number of patients with pulmonary contusions per year (from 2.73 to 8.27), increase of injuries in males (from $56 \%$ to $70 \%$ ), reduced need for tube thoracostomy (from $39 \%$ to $12 \%$ ), and reduced number of days spent in the hospital(from 25 to 19 days) when the time period 1986-2000 was compared to 2001-2011, with no significant differences in ISS and mortality. Whileduring 1986-2000 no pulmonary contusions were caused by moped accidents, during 2001-2011 moped accidents have been the second most common cause of thoracic injury. 
Conclusion:The increased number of patients with pulmonary contusion per year and the emergence of moped accidents as one of the main mechanisms leading to pulmonary injuries during the 2001-2011 time period may be seen as local phenomenon, but certainly intrigue for further more global research.

Keyword; Chest trauma in children, Pulmonary contusions, thoracic injuries

\section{Outcomes and Treatment of Traumatic Hemothorax: Analyses our Cases.}

Fadil GRADICA ${ }^{1 *}$, Agron DOGJANI ${ }^{2}$, Lutfi LISHA $^{1}$, Dhimitraq ARGJIRI ${ }^{3}$, Alma CANI ${ }^{4}$, Fahri KOKICI ${ }^{4}$, Ylber VATA ${ }^{1}$, Shkelzen OSMANI $^{2}$, Afron MICI ${ }^{2}$, Skender BUCI ${ }^{2}$, Ylli ZIQISHTI ${ }^{1}$.

${ }^{I}$ Thoracic and visceral Surgery Service, University Hospital "Shefqet Ndroqi" Tirana, Albania.

${ }^{2}$ General Surgery and Trauma Service, University Hospital of Trauma, Tirana, Albania.

${ }^{3}$ Pneumology Service, University Hospital "Shefqet Ndroqi" Tirana, Albania.

${ }^{4}$ Anesthesie Reanimation Service, University Hospital "Shefqet Ndroqi" Tirana, Albania.

\section{Abstract}

Background: Guidelines for the management of traumatic hemothorax are well established.

The purpose of study: To assess the management and outcomes of patients with traumatic hemothorax.

Material and methods: We conducted a retrospective study of all trauma patients diagnosed with a hemothorax in University Hospital of Trauma, and University Hospital "Shefqet Ndroqi" Tirana Albania from January 2010 to January 2018. Results: Sixty-five patients with traumatic hemothorax were identified, 41 from blunt mechanism and 24 from penetrating mechanism. The majority of patients injured by penetrating mechanisms were treated with tube thoracostomy and 10 thoracoscopy (VATS) or 17 thoracotomies two cases Clamschell approach while the majority of blunt injury patients were observed, pt tube thoracostomy. Among patients suffering from blunt mechanism, patients who were managed with chest tubes had a greater volume of hemothorax than those who were observed. All patients who were observed underwent serial chest radiographs demonstrating no progression and required no delayed procedures. Patients with a hemothorax identified only by computed tomography, after negative plain radiograph, did not require intervention. Three patients developed a delayed empyema one patient and two patients fibrothorax.

Conclusion: Patients with massive thoracotomy hemothorax and clamschell aproach are safety for the survival of patients with complications and low mortality whereas a small-volume hemothorax may be safely observed without mandatory tube thoracostomy and with overall low complication rates.

Key Words: Hemothorax, blunt trauma, penetrant, tube thoracostomy, VATS.

\section{Chest Wall and Sternal Resectionfor Primary or Secondary Tumors, our Experience in Single Unit in Albania.}

Fadil GRADICA ${ }^{1}$, Lutfi LISHA ${ }^{1}$, Dhimitraq ARGJIRI $^{2}$, Alma CANI ${ }^{3}$, Fahri KOKIÇI ${ }^{3}$, Lutfi ZHEGU $^{1}$, Agron MENZELXHIU ${ }^{1}$, Astrit KALENJA $^{1}$, Ibrahim AVDIU ${ }^{4}$, Helidon NINA ${ }^{4}$, A. $\mathrm{PERE}^{4}$, D. XHEMALAJ ${ }^{4}$

${ }^{I}$ Thoracic and visceral Surgery Service, University Hospital "Shefqet Ndroqi" Tirana, Albania.

2 Pneumology Service, University Hospital "Shefqet Ndroqi" Tirana, Albania.

${ }^{3}$ Anesthesia And Intensive Care Service, University Hospital "Shefqet Ndroqi" Tirana, Albania.

${ }^{4}$ Oncology Service, University Hospital Center

"Mother Theresa"Tirana, Albania.

\section{Abstract}

Background: Chest wall resection involves resection of the ribs, sternum, costal cartilages and the accompanying soft 
tissues and the reconstruction strategy depends on the site and extent of the resected chest wall defect.

Materials and Methods: The study includes 75 patients (46 male and 29 female, rate M/F 2:1). that underwent chest wall resection and reconstruction with prosthetic material at the University Hospital "Shefqet Ndroqi" ,thorax surgery division during december 2013 - december 2017 timeframe.The median age was 57 years and ranged from 16 to 73 years. The indication for resection was primary chest wall and sternal malignancy in $45(60 \%)$ patients; 20 (26.6\%) patients osteosarcom; 7 (9.3\%) patients condrosarcom; 5 (6.7\%) solitary plasmocitoma; 4 (5.3\%) desmoidal tumor; 2 (2.7\%) patient liposarcoma; 2 (2.7\%) patients Ewing sarcoma; 2 (2.7\%) patient hemangio-pericitoma; rabdomyosarcoma $2(2.7 \%)$ patients; $1.3 \%$ patient malignant non Hodgkins lymphoma), secondary (solitary chest wall metastase )and recurrent chest wall malignancy in $11(14.7 \%)$ patient, contiguous lung cancer (NSCLC) in 11 $(14.7 \%)$ patient, breast cancer recidive in $8(10.7 \%)$ patients.

Results: Skeletal reconstruction was achieved with prosthetic material alone in $35(46.7 \%)$ patients and with Prosthetic materials covered by flaps of myocutaneous or muscle tissue were used (Prolen mesh and methyl methacrylat sandwich plastic) in 26 (34.7\%) patients, myocutaneous or muscle flaps alone in 14 $(18.7 \%)$. Soft tissue coverage was achieved with transposed muscle in 48 (64\%) patients, local tissue only in 27 (36\%) patients...

Conclusions: Our experience demonstrates that sternal and chest wall resection is a safe and effective treatment, which may improve the patient survival and quality of life.

Keywords: Chest wall resection; primary and secondary malignancy, reconstruction
143. Culdotomy as a Minimally Invasive Surgical Procedure with Tubectomy for Definitive Sterilisation and in Tubal Pregnancy.

Arben HAXHIHYSENI ${ }^{1 *}$, Daniela VERCUNI ${ }^{1}$. ${ }^{I}$ Gynecology Service, Durres Regional Hospital, Albania.

\begin{abstract}
Background: Transvaginal surgery is an earlier surgical procedure than laparoscopy. In the Regional Hospital of Durres, Gynaecology Service, it was applied for the first time in 2007, and since then it became an usual procedure.

Objectives:To evaluate the efficacy and safety of definitive transvaginal tubar sterilisation and tubectomy in tubar pregnancies (ruptured or not).
\end{abstract}

Methods: This is a cohort study which includes 58 cases of definive transvaginal tubar sterilisation and 37 cases of tubectomy in tubar pregnancies ( 23 ruptured and 14 not ruptured). The study started in January 2007 and ended in December 2017. In each case the procedure was explained to the patient and their approval was mandatory.

Results: Mean time for definive transvaginal tubar sterilisation was 4.6 min. and for tubectomy 13.9 min. Mean blood volume lost in unruptured cases was $65 \mathrm{ml}$ and $278 \mathrm{ml}$ in ruptured cases. Mean time of hospitalisation was between 12 to 24 hours. Only one case with ruptured tubar preganancy, which was complicated with hemoperitoneum, was hospitalised for four days. Total rehabilitation was made in 6 days. There were no complications for a one year period.

Conclusions: Culdotomy is a safe procedure to make definive tubar sterilisation or to make tubectomy in tubar pregnancies. Based on cost - effectiveness of this procedure we recommend it to be used more in Albaninan Gynaecology. Key words: Culdotomy, Transvaginal approach surgery, transvaginal tubectomy 


\section{Placental Abruption; Proper Evaluation and Management}

\author{
Genc KABILI ${ }^{1}$, Rustem CELAMI ${ }^{2}$, Danny \\ DAPHNIS $^{3}$ \\ ${ }^{1}$ Obstetrician and Gynecologist, American Hospital \\ of Tirana, Tirana, Albania \\ 2. Obstetrician and Gynecologist with Hospital \\ Priveledges at Elbasan Regional Hospital and \\ American Hospital; Professor at Faculty of Medical \\ Technical Sciences “Alekander Xhuvani”, Elbasan, \\ Albania. \\ ${ }^{3}$ Scientific Director of the IVF Centre - \\ Mediterreanean Fertility Institute, Crete, Greece
}

\begin{abstract}
About 1 in 100 pregnant women, or $1 \%$, have placental abruption. It usually happens in the third trimester, but it can happen any time after 20 weeks of pregnancy. Mild cases may cause few problems. An abruption is mild if only a very small part of the placenta separates from the uterus wall. A mild abruption usually isn't dangerous. Severe placental abruption, greater separation between the placenta and the uterus, the baby is at higher risk for; growth problems, premature birth, stillbirth, death, or lasting disabilities. Signs and symptoms of placental abruption should be weel known by all medical staff working with pregnancy care, as early diagnosis is essential. Such medical problem during pregnancy poses an emergency and should be evaluated, diagnosed and propered managed in order to reduce fetal and maternal morbidities and mortalities.
\end{abstract}

Keywords: Placenta, abraption, fetus, morbidity, mortality, diagnosis, management.

\section{Arterial catheterization in post partum hemorrhage (PPH). A therapeutic alternative in modern obstetrics.}

${ }^{1}$ American Hospital Nr.3, Tirana, Albania

\begin{abstract}
Intra and post partum hemorrhage are lifethreatening emergencies, a common causes of maternal mortality-morbidity and still remain a great treatment challenges in modern obstetrics. PPH is the first cause of deaths in developing countries while in western countries is thromboembolism. $\mathrm{PPH}$ is defined as a blood loss of $500 \mathrm{ml}$ or more after birth or severe PPH as blood loss of $1000 \mathrm{ml}$ or more. Another classification of $\mathrm{PPH}$ is time related, into primary (within 24 hours of delivery) and secondary (between 24 hours and six weeks post partum.

At term maternal blood volume is approximately 7 litres and blood loss may be life-threatening with unreplaced volume loss of as little as $30 \%$.

Association of maternal anemia with postpartum hemorrhages appears to be important not only as a predisposing factor for parturient but also reducing resistance to blood losses.

$\mathrm{PPH}$ is closely related to surgical interventions as episiotomy, operative vaginal delivery or c-section Uterine or hypogastric artery catheterization is a life-saving therapeutic alternative and preserves future fertility at the same time. Main indications are post partum uterine atony, placenta previa, placenta accreta, persistent bleeding after cesarean section and hysterectomy, genital tract trauma, bleeding disorders, ectopic pregnancy etc.
\end{abstract}

\section{Title Surgery versus metotreksat treatment of ectopic pregnancy in $\mathrm{UHC}$ \\ Queen Geraldine between 2010 and 2015.

\author{
Maksim GJONI ${ }^{1}$ \\ 1 "Queen Geraldine", University Hospital Center, \\ Tirana, Albania
}

\section{Abstract}

This study can be used as a reference for the treatment of EP in Albanian population 
according to the equipment we use and the population profile.

We have been changing gradually from traditional and the only way of treating EP to the pharmacological approach in selected cases. Later on endoscopy was available and we will include this in a later approach of the problem.

In this study we followed $228 \mathrm{EP}$.

Including and excluding criteria were established for each method of treatment. Diagnosis and follow up was achieved by using ultrasound and bHCG, culdocentesis vaginal bleeding, pain. During the study as early as possible diagnosis was attempted. In this study we looked for differences in the parity, age of the pregnancy, former surgery, abdominal liquid, operative technique, hisopathological examination, cost effectiveness.

We documented soma complication such as bleeding, pain, future fertility,

Key Words: ectopic pregnancy, surgery, metotreksat,

\section{Urinary Tract Injury During Hysterectomy}

Leon $\mathrm{KAZA}^{1}$, Rustem CELAMI ${ }^{2}$

${ }^{1}$ Obstetrician and Gynecologist, Lezha Regional Hospital, Lezha, Albania

${ }^{2}$ Obstetrician and Gynecologist, Elbasan Regional Hospital and American Hospital of Tirana, Tirana, Albania;

Professor at Faculty of Medical Technical Sciences, U. of Elbasan “A. Xhuvani”, Elbasan, Albania

\footnotetext{
Abstract

Hysterectomy is the most common gynecologic procedure performed mostly for benign indication. The reproductive and urinary tracts in women are closely related anatomically and embryologically. Urinary tract injury during hysterectomy is not uncommon. Injury to the urinary tract involves injury to the ureters, bladder and urethra. The combined incidence of lower urinary tract injury during different modes of hysterectomy is reported to be as high as $4.8 \%$. Bladder injury is approximately three times more common than ureteral injury. Ureteral injury is one of the most
}

serious complications of gynecologic surgery associated with significant morbidity and the potential loss of kidney function, especially when not recognized until postoperatively. For these reasons, injuries to the urinary tract, particularly the ureter, are the most common cause for legal action against gynecologic surgeons. Urological injuries in gynecological surgeries result due to difficult or careless surgery and/or associated with active infection, endometriosis, enlarged uterus, pelvic adhesions, distorted pelvic anatomy, previous radiation.

In our presentation we will review how and why lower urinary tract injuries occur during hysterectomy. A number of cases of lower urinary tract injuries during hysterectomy performed at Lezha Regional Hospital will be discussed. The question of training of gynecologist as e pelvic surgeon will be addressed in our speech. Gynecologist has to be conscious of the possibility of urological injuries and take preventive steps but should not be frightened and pushed to inaction. In conclusions, preventing urinary tract injury during gynecologic procedures needs a well established strategy which requires knowledge of anatomy, risk assessment and intraoperative srcreening to recognise and repair such injuries.

Keywords: urinary tract injury, urinary tract fistula, hysterectomy, bladder, gynecologic surgery

\section{Newborn Trauma: Causes, Neonatal Management and Prevention.}

Elona GJYLBEGU ${ }^{1}$, Niketa KOLICI ${ }^{1}$, Vangjush LEKGJIKA $^{1}$, Klodian HYKA ${ }^{1}$

${ }^{I}$ Neonatal Department, American Hospital, Tirana,Albania.

\begin{abstract}
A variety of traumatic lesions can occur during the neonatal period. Some of those lesions are clearly birth injuries due to delivery and others are caused by necessary procedures during intensive care in critically ill neonates. As usual patient
\end{abstract}


history must be known and knowledge about the typical complications is necessary in order to select the appropriate imaging modality and thus enabling correct interpretation of those investigations by the radiologist.

Most birth traumas are self-limiting and have a favorable outcome. Nearly one half are potentially avoidable with recognition and anticipation of obstetric risk factors. Infant outcome is the product of multiple factors. Separating the effects of a hypoxic-ischemic insult from those of traumatic birth injury is difficult.

Risk factors for birth trauma include the following:

- Large-for-date infants, especially infants who weigh more than $4500 \mathrm{~g}$

- Instrumental deliveries, especially forceps or vacuum

- Vaginal breech delivery

- Abnormal or excessive traction during delivery

- Abnormal or excessive action during neonatal resuscitation

Recognition of trauma necessitates a careful physical and neurologic evaluation of the infant to establish whether additional injuries are present. Symmetry of structure and function should be assessed, the cranial nerves should be examined, and specifics such as individual joint range of motion and scalp/skull integrity should be evaluated.

Prognosis; Not all birth injuries will require surgery. However, in some instances, severe injuries may require surgery in order for the infant to have the best chances of survival. A wide variety of medications are used to treat birth injuries. As with surgery, not all birth injuries require medications. In many cases, however, medication is prescribed for pain, seizures, cognitive disabilities, and more.

\section{Critical Obstetrical Emergencies; How Prepared Are We? A Case Report}

Rustem CELAMI ${ }^{1}$, Leon KAZA ${ }^{2}$, Genc KABILI ${ }^{3}$, Anton $\mathrm{MILO}^{3}$, Zef DELIA ${ }^{4}$, Agron DOGJANI ${ }^{5}$

${ }^{1}$ Professor in Obstetrics and Gynecology, Faculty of Medical Technical Sciences, University of Elbasan " $A$.

Xhuvani”; Hospital Privileges as Obstetrician and Gynecologist at Elbasan Regional Hospital and American

Hospital of Tirana, Tirana, Albania.

${ }^{2}$ Obstetrician and Gynecologist at Lezha Regional Hospital, Lezha, Albania.

${ }^{3}$ Obstetrician and Gynecologist, American Hospital of Tirana, Tirana, Albania

${ }^{4}$ Professor in Obstetrics and Gynecology, Faculty of Technical Medical Sciences, University of Elbasan " $A$.

Xhuvani”; Obstetrician and Gynecologist, American Hospital of Tirana, Tirana, Albania

${ }^{5}$ Professor of Surgery, Faculty of Medicine,

University of Medicine of Tirana; General Surgeon at Trauma

University Hospital, Tirana, Albania

\begin{abstract}
Background: Medical emergencies may occur at any time in any setting, particularly the inpatient setting. It is essential that obstetrician gynecologists prepare themselves by assessing probable emergencies, establishing early warning systems, designating specialized first responders, conducting emergency simulations, and debriefing staff after actual events to identify strengths and opportunities for improvement. Having such systems in place may reduce or prevent the severity of medical emergencies.
\end{abstract}

Case report; A 28 years old, pregnant woman, in her third term pregnancy, comes to Maternity Service, Elbasan Regional Hospital, in late evening, with broken waters at home and she came within few minutes to the hospital. The admission midwife performs routine bimanual pelvic examination and detects the fetal umbilical cord proceeding fetal head. The midwife calls and informs the obstetrician and gynecologist on duty, meanwhile, she had taken the hand out, and when physician on duty performs 
respective bimanual pelvic examination the umbilical cord was touched by gloved fingers - proceeding fetal head, and immediately a maneuver with two fingers was tried to push up in a spiral circle the fetal umbilical cord that was just around the head, and at the moment the head felt like was tighten to cervix uteri with dilation 5-6 cm....

In conclusions, obstetrical emergencies, and emergent obstetrical surgeries are present in every day maternity/hospital practice. Skilled staff can save the fetal and maternal lives if they are well prepared and infrastructure is as needed. Emergency simulations are needed in order to assess and evaluate the performance in such cases and prepare for better management of such emergencies.

Keywords: Obstetrical emergencies, skilled medical staff, infrastructure, emergency simulations

\section{Spinal Anesthesia and its Role in Orthopedic Surgery of the Lower Extremities}

Elona HASALLA $^{1 *}$, Ilia MAZNIKU ${ }^{1}$, Ilda TAKA ${ }^{1}$, Blerta HASALLA ${ }^{1}$, Sulejman BAHA ${ }^{2}$

${ }^{I}$ Faculty of Technical Medical Sciences; University "Aleksandër Xhuvani" Elbasan, Albania

${ }^{2}$ Department of Pre-Clinic Subjects, Environmental and Diagnostic Research Center, University of Florence, Italy

\begin{abstract}
Introduction; Spinal anesthesia involves the use and application of local anesthetic agents in the subarachnoid space to benefit from a reversible loss of sensory and motor function.

The purpose of our study is to underline the value and guarantee of spinal anesthesia in creating optimal, painless and no side effects in patients who need interventions below Th.5. and to present our modest experience and encourage its use in orthopedic operations in the cases of traumas of lower extremities.
\end{abstract}

Material and Methods; 800 patients, 720 males $(90 \%)$ and 80 females (10\%) were taken into study, with lower extremity lesions in the time-period from November 2016 to August 2018. The patients were all above 18 of age, $40 \%$ of patients (320) were presented in emergency conditions, with eating and other associated complications, while $60 \%$ of patients (480) were scheduled operations.

The maximum age was 87 years, the average age 42 years and the youngest age 14 years. The medicines used were Lidocaine ( $2 \%-3 \mathrm{ml}), 760$ patients $(95 \%)$, $1.5-2 \mathrm{~h}$ action time and marcaine (2\% $2 \mathrm{ml}), 40$ patients $(5 \%), 2.5 \mathrm{~h}$ action time. Conclusions; Finally, we recommend the use of spinal anesthesia in orthopedic surgery in the case of inferior traumas, as the complications are minimal and the patient's benefits are considerable.

Key words: spinal anesthesia, trauma of lower extremities,lidocaine, marcaine

\section{The Role of the Physiotherapist in the Emergency Department}

Erisa MANE ${ }^{1}$

${ }^{I}$ Department of Diagnostics and Health Rehabilitation,

Faculty of Technical Medical Science, University of Medicine Tirana, ALBANIA

\section{Abstract \\ Background: Emergency Department (ED) is the department of a hospital responsible for the provision of medical and surgical care to patients arriving at the hospital in need of immediate care. ED personnel may also respond to certain situations within the hospital such cardiac arrests. The role of advanced musculoskeletal physiotherapists is still new and evolving. Aim: The main purpose of the study is to identify the role of physiotherapist in ED as a necessity of the future. Specific objectives that were achieved through this study are: Impact of physiotherapist in the ED on patients with neck and back pain. Primary assessment and treatment of patients with musculoskeletal injuries by physiotherapists in ED. Comparison of standard care with physiotherapeutic management}

Materials and Methods: The study is a Systematic Review, meta-analytic type, consisting of 7 studies of the last ten years, 
which focus on the impact of physiotherapy in ED. Studies include 1255 patients evaluated and treated by ED physiotherapist in primary contact compared to secondary and standard care. The evalution includes the patient's condition, disability, pain, waiting time, treatment, length of stay and patient feedback to the staff.

Results: The literature used in the study claimed that physiotherapist in ED reduces pain and disability 4 times faster than standard care and reduces length of stay by an average of 40 minutes. Over $82 \%$ of patients and $96 \%$ of staff agreed on physiotherapist as primary care in the Emergency Department.

Conclusions: The new Emergency Care model includes advanced musculoskeletal physiotherapist for assessing and treating patients with minor injuries. Their work is has proven to be effective in reducing waiting times, achieving higher patient satisfaction rates and improving the patient's overall condition. There is a need for specific training of physiotherapists and staff reorganization of the Emergency Department.

Keywords: Emergency Department, Advanced Musculoskeletal Physiotherapist

\section{Use in Clinical Practice by Nursing Staff of Proper Body Mechanics: Cross-Sectional Study}

Gentjana HASKJA $^{1^{*}}$, Fatjona KAMBERI ${ }^{1}$

${ }^{1}$ Public Health Faculty, "Ismail Qemali” University of Vlora, Albania practice and the presence of problems associated with its non-implementation. Materials and Methods: This crosssectional study was conducted at the Vlora Regional Hospital in November 2017 February 2018. 53 out of 54 nurses participated in the study. The nursing staff's knowledge of body mechanics and it's practice was evaluated by a structured literature questionnaire.

Results: Most nurses were women $(\mathrm{n}=38.72 \%)$ aged $25-30$, while men were $15(28 \%)$ aged $31-35$ years. Most of them had a working experience of 5-10 years ( $\mathrm{n}=21,40 \%)$. Of the 53 nurses surveyed, $\mathrm{n}=33(62 \%)$ were graduates in a master's degree. From the analysis of the connection between the knowledge and the department where nurses work, there is a significant statistical difference between nurses in different departments in relation to the level of the nurses $(\mathrm{p}=0.0019$, $<0.05$ ).

Conclusion: The results showed that most nurses had knowledge and practice of proper body mechanics as well as previous backache experiences. Educational programs to increase the awareness of nursing staff about the importance of proper use of bodywork mechanics during work are essential to avoid problems associated with the lack of proper body mechanics.

Keywords: knowledge, practice, works safety, nursing staff, and body mechanic.

\section{Musculoskeletal Disorders that Appear in Nurses of the Surgery Department regarding work Conditions and Psychological Load}

Julian KRAJA ${ }^{1}$

${ }^{1}$ University "Luigj Gurakuqi” Faculty of Natural Sciences, Department of Nursing, Shkoder, Albania 
work due to occupational injury and illness. Muscular-skeletal injuries are expensive for both individuals and industry. The American Nurses

Association estimates $\$ 7$ billion U.S. dollars annually which are spent in direct (medical bills) and indirect (worker's compensation and staff replacement) costs. The purpose of this paper is to identify the muscular-skeletal problems observed in nurses during their work by linking to demographic factors, psychological demand and control over work in the surgery department.

Material and methodology: This study was conducted in the district of Shkodra, were there examined nurses working in the surgery department, Regional Hospital Shkodra.

Results In total, 18 nurses working in this department were interviewed. The questionnaires used are: Job Content Questionnaire dhe Nordic Muscularskeletal Questionnaire. The study was conducted during May-Jun 2018.

Conclusions: Nurses have a considerable stress level in solving the various problems they have during their work. This is reflected in the answers to the questions they have agreed in order to carry out their work. They need maximum focus on the work they do, as well as the high demand for quick action with great skill. The results indicate that nurses refer to pain in different parts of the body affecting their daily activity.

Keywords: nurse, surgery, psychosocial, Muscular-skeletal.

\section{Post-Factum Evaluation of Radius Fractures in the Typical Place in Relation to Age, Gender, Osteoporosis Rate, and Treatment Outcome.}

Elona HASALLA ${ }^{1}$

${ }^{1}$ University Aleksander Xhuvani, Faculty of

Medical and Technical Sciences-Elbasan, Albania

Abstract
Introduction:
It is well known that the underlying mechanisms of osteoporosis in older adults are different than those associated with estrogen deprivation. Age-related bone loss involves a gradual and progressive decline, which is also seen in men. The aim is to evaluate the influence of the above fractional on the frequency of radius fractures in the typical place, particularly of osteoporosis as the main cause of these fractures at the age of three. Functional treatment results.

Patients and Methods; Actually 82 patients with radial fracture in the typical place of conservative treatment, implantation and implantation in gypsum, as well as the respective reactive rehabilitation procedures were studied. In the material, they have taken a survey; the age of the patients, the sex, the rate of osteoporosis, the technique of reproduction, how long after the fracture and eventually the results achieved. They were 76 women or $92.6 \%$ and 6 males or $7.4 \%$. The maximum age of 86 years of age, the average age of 75 and the minimum age of 63 In the study, primary is the rate of osteoporosis as potential potential for radius fractures in the typical place.

Results: In our study series, the largest number of patients was female, eighty-two cases or $92.6 \%$ of the study group, the rest of $7.6 \%$ or 6 cases were mammal. The study period was 2 years of 2016-2017 all fractures are in traumatic terrain.

Traumatic trauma in $60 \%$ of cases has been overturned or otherwise reported as a direct trauma, whereas in $40 \%$ of cases there has been an indirect trauma. pain, loss of function, different hematoma rates as well as radiological changes were the signs of these injuries. Treatment endpoints were favorable especially when the implantation period of three weeks was respected in gypsum and the beginning of rehabilitation exercises

Conclusion: Typical site radius fractures are heavily influenced by regional osteoporosis, age of patients, osteoporosis rate. Generally, benign fractures tend to 
consolidate. A three-ëeek period is sufficient for the attachment of bone fragments and the origin of rehabilitation. Over-term complications, such as dysfunctional bone atrophy, are more difficult to treat than fractures themselves. Keywords: osteoporosis, aging, elderly, osteoblast, osteoclast, treatment

\section{Nursing Management of the Patient with Disability after Craniotomy}

Kimete KADESHA ${ }^{1}$, Naime ÇULLHAJ ${ }^{2}$, Irena TRESA $^{2}$, Albenc DERVISHI ${ }^{3}$

${ }^{1}$ Chief nurse, Neuroscience Department of UHCT

"Mother Theresa", Tirana, ALBANIA

${ }^{2}$ Head Nurse, Neurosurgery Unit of UHCT

"Mother Theresa", Tirana, Albania

${ }^{2}$ Head Nurse, Neurosurgery Unit of UHCT

"Mother Theresa", Tirana, Albania

${ }^{3}$ Nurse, Neurosurgery Unit of UHCT "Mother

Theresa", Tirana, Albania

\section{Abstract}

There are $20 \%-25 \%$ of the patients hospitalized in the neurosurgical department, consequently of brain tumors and neurovascular disease, they suffer cranial nerves damage, motor deficits of the extremities and consciousness's alteration, after craniotomy.

These elements make the patients uncollaborative and depended from the paramedical staff.

The role of the nursing care is: 1 .

Identifying needs, 2. Perform the general nursing care protocols, 3 . Perform the specific nursing care protocols according the needs.

When the patient is admitted in the hospital. it is important, collaboration with the team of doctors, nurses, rehabilitation team. We gather the data to know situation of the patient from the beginning till the moment. The data serve us to develop nursing care plan, to prevent complications .

This is a study toward groups of patients that have experienced neurological disability, low karnofsky scale, after craniotomy and nursing care according specific needs of the patients.

Neurosurgery service has a very good experience that shows the patients after surgery, life has been saved. The patients that developed neurological disability after surgery , are taken on charge $100 \%$ from the staff to fulfill their needs. A good education is given to their families. high level monitoring of the patients and high level of nursing care, decrease complication and handicap of them.

Keywords: Disability, daily life activities, needs priority, Karnofsky scale, consciousness level, complication prevention, rehabilitation.

\section{Considerations about Gestational Diabetes}

\author{
Natasha MERKO ${ }^{1}$, Lindita SAJA ${ }^{1}$, Elvi SOTA ${ }^{1}$, \\ Klejdi LATIFI ${ }^{1}$ \\ ${ }^{I}$ Nurse Service, University Hospital of Trauma, \\ Tirana, ALBANIA.
}

\begin{abstract}
Background: "Gravidanza" is a special condition of the mother during which a creature is developing in the body. The duration of pregnancy is based on the age of pregnancy which is calculated from the first day of the last menstruation, accepting a 28-day cycle. The pregnancy is expressed in full weeks. A fetus in the term is considered in the term he born alive in 3840 weeks, premature before 37 weeks and a fetus serotine or post term is considered a fetus born alive after week 42 of pregnancy. Gravidanca manifests itself with signs and symptoms, combined with gynecological and imaging examinations, decide the diagnosis.
\end{abstract}

Aim: This study is designed to sensitize and educate all mothers who need to have a better understanding of the complications and illnesses that may occur during pregnancy that endangers two lives, that of the mother and the fetus.

Materials and Methods: This study is a retrospective study, in both Maternity " 
Koço Gliozheni" and "The Queen Geraldine" in Tirana, on cases of patients diagnosed with gestational diabetes.

Conclusions: Genetic Diabetes is a complication, a very dangerous metabolic disorder in pregnancy, endangers both life and maternal and fetal risk, increases the risk of neonatal disease and morbidity. In this study, it is reflected that a special care of the multidisciplinary team in preventing it with early diagnosis and good care during pregnancy at its beginnings, and an effective treatment of this pathology, eliminates the risk of Amtaro-fetal, but also neonatal.

Keywords: Gravidanza, Diabetes, Glucose, Complications, Prenatal Diagnosis.

\section{How Is Affects Application of Mezieries Method in Idiopathic Scoliosis. A Case Study.}

Selda VEIZAJ ${ }^{1}$, Aida LAKO $^{1}$, Irena KOLA ${ }^{2}$, Enkeleda SINAJ ${ }^{2}$

${ }^{1}$ Faculty of Professional Studies, University

“Aleksander Moisiu “, Durrës, Albania,

${ }^{2}$ Fakulty of Technical Medical Science, University of Medicine of Tirana, Albania

\begin{abstract}
In this case study for idiopathic scoliosis is the evaluation of the potential of the Mezieries Method in a patient with idiopathic scoliosis, in which he has not yet finish the maturity skeletal. What impact does this method have on stabilization of Cobb's angle?

Clinical Case; One 15-year-old girl, who was born in October 2003, was diagnosed with structural scoliosis that presented with a right thoracic curve of 17.8 degrees Cobb angle and lumbar curve of 36.9 degrees Cobb angle. The patient was diagnosed 3 years ago with scoliosis, and just now she have undergone physiotherapy.

Physiotherapeutic intervention; The girl was undergone to regular sessions of physiotherapy for a period to one year and a half, realizing two sessions a week. For 23 hours of the day she keeps the personalized brace, which is recommended by the orthopedic doctor. She has not yet
\end{abstract}

received the recommendation of the orthopedic doctor for surgery, regardless the fact that there are some schools in Germany which advise for surgery even without reaching the curve of fifty degrees of scoliosis. For the rehabilitation period, during clinical and radiological examinations no one has ever been noticed improvements of the Cobb angel but not even any deterioration of it.

Conclusions; Mezieries therapy was observed in our patient, help not aggravation of the Cobb angel, despite the fact that over she has not completed skeletal maturation. We cannot say it was effective in reducing the Cobb angel, however we suggest that a significant reason to not reduce the Cobb abgel was the personalized brace shape. In our opinion, the brace demanded modifications Keywords; Therapy of Mezieries, physiotherapy, idiopathic scoliosis

\section{Traumatic and Non- Traumatic Injuries. Surgery and Rehabilitation}

Zojë DEMAJ $^{1}$

${ }^{1}$ Nurse, Salus Hospital, Tirana, Albania

\begin{abstract}
Aim: This study aims to describe the traumatic and non-traumatic injuries of articulations and bones as well as medical care, surgery and rehabilitation after surgery. The study accentuates nursing care and the importance of physiotherapy in recuperation proccess.
\end{abstract}

Materials and Methods: Between January 2016 and December 2017 we analyzed a total of 335 patients treated in the department of orthopedic surgery of a private hospital in Tirana. All patients were 16-90 years old. According to our Hospital protocols (keeping the privacy of all patients) we analyzed the data of patient regarding the diagnosis of hospitalization (cause of injuries), age, gender distribution. 
Results: All of 335 patients taking the study result that the largest part are male (195 of 335) and the others female (140 of 335) .Regarding to the type of injuries and intervention, result that 82 of the 335 cases presented are for ACL reconstruction ,for a diagnostic artroscopy (knee pain) are 116 cases presented from 335 patients, for meniscopaty and rupture of menisc are 90 cases presented from 335 . The patient presented in the hospital for hip fracture were 15 of 335 cases and they had done a total hip replacement. Other cases presented were : gonartrosis 27 of 335 cases (total knee replacement surgery) and artroscopy of shoulder 12 of 335 cases. Conclusions: At the end of the study, we found that the most numerous cases presented to the hospital are due to sports trauma, home incidents (old age), and degenerative bone diseases.

Keywords: ACL, trauma, hip fracture, coxartrosis, gonartrosis, artroscopy, surgery, meniscopaty, nursing care, physiotherapy.

\section{Internal Fixation of Osteoporotic Bone}

Jordan SAVESKI ${ }^{1}$, Neda TRAJKOVSKA ${ }^{1}$, Ilir $\mathrm{HASANI}^{2}$.

${ }^{1}$ Clinical Hospital Acibadem Sistina, Skopje, Macedonia

${ }^{2}$ University Clinic of Traumatology, Medical Faculty, Skopje, Macedonia

\section{Abstract}

Osteoporosis is asymptomatic disease; occurrence of fragility fracture is often its initial manifestation. This condition which is characterized by decreased bone density and thinning of cortical bone is strongly influenced by complex signaling in both hormonal and mechanical environments. Many of the traditional fixation constructs used for repair of these fractures are not suitable for use in osteoporotic bone. Increased use of new implants locking plates with fix or variable angles, intramedullary devices and bone substitutes has greatly improved outcomes in these patients. The spine, wrist, proximal humerus, hip and distal femoral and proximal tibial fracture are common sire of osteoporosis in elderly persons. Beside the proper choice of the new implants and tactic in fixation of osteoporotic bone the surgeon has to decide about the augmentation of healing and stability of fracture. The best augmentation is autogenous cancellous bone graft (ACBG), which is the best material for bone grafting because of the following: Contains all of the ingredient necessary to stimulate bone healing, osteoconductive matrix, osteoinductive signaling molecules and cells capable of responding to these signals and $80 \%$ of the osteogenic capacity is the result of mesenchymal stem cells within the harvested bone. Beside ACBG there are many bones and substitutes biologic materials in use for healing of osteoporotic bone. PMMA cement was first used to augment fixation of unlocked condylar buttress plate fixation in supracondylar femoral fracture However, these methods are limited in that PMMA induced thermal necrosis which results in screw loosening and PMMA cement does not support or promote new bone formation. PMMA is not an ideal material for this purpose because it becomes a permanent implant and foreign body within bone.

How to make the proper choice of the fixation construct and augmentation of fracture healing and stability is the purpose of this presentation.

\section{Biologically Enhanced Anterior Cruciate Ligament Reconstruction}

\author{
Alan ANDONOVSKI ${ }^{1 *}$, Ilir HASANI ${ }^{1}$, \\ Andonovska B ${ }^{1}$, Ilir SHABANI ${ }^{1}$ \\ University Clinic for Traumatology, Orthopedic \\ Surgery, Anesthesiology and Intensive Care. \\ Faculty of Medicine, University "Ss Cyril and \\ Methodius” Skopje, Macedonia.
}
Abstract
Introduction: Although the improvement of Anterior Cruciate Ligament (ACL) reconstruction surgical techniques, ACL 
reconstruction is not a " $100 \%$-success" procedure. Current research is investigating new methods to biologically enhance graft tissue healing, providing superior clinical results with low failure rate after ACL reconstruction.

Objective: The purpose of this study was to evaluate the influence of ACL residual remnant and Plasma Preparation Rich in Growth Factors (PRGF) on graft healing and postoperative clinical results after ACL reconstructive techniques.

Methods: Eight patients (mean age 25) with ACL rupture and intact ACL residual remnant with diameter more than $1 / 3$ of the intact ACL were included in this study. The patients were equally divided into 4 groups: Group I (control group) with single bundle ACL reconstruction; Group II with ACL reconstruction and application of PRGF; Group III with remnant preserving ACL reconstruction and Group IV with remnant preserving ACL reconstruction and application of PRGF.

Results: Regarding the presence of synovial fluid at the bone-graft interface, completed graft integration within the bone tunnels was seen in $30 \%$ of the patients in group I, 37\% in group II, 55\% in group III and $57 \%$ in group IV. Regarding the graft signal, finished graft maturation was found in $35 \%$ of the patients in group I, $57 \%$ in group II, $75 \%$ in group III and $85 \%$ in group IV.

Conclusion: Preserved ACL residual remnants have beneficial effect on graft maturation and graft integration within the bone tunnels evaluated on MRI. They prevent tibia tunnel widening and obtain better anterior knee stability. PRGF has an enhancing effect on graft maturation and showed synergic effect on prevention of tibia tunnel widening and postoperative knee stability. This study showed the importance in preserving ACL residual remnants and application of PRGF in patients after ACL reconstruction.

Keywords: ACL reconstruction, ACL residual remnant preservation, Preparation Rich in Growth factors, Graft healing, Clinical results.

\section{Post Traumatic Patellar Absence in Knee Endoprosthesis. Case Reports}

\author{
Dritan TODHE ${ }^{1}$ \\ ${ }^{1}$ Orthopaedic and Trauma Service, University \\ Hospital of Trauma, Tirana, ALBANIA
}

\begin{abstract}
Background: Patients with previous patellectomy are difficult cases and reconstruction of the joint with adequate stability is a challenge to every surgeon. Patellectomy results in a change in the alignment of the quadriceps and patellar tendons and may aggravate anteroposterior instability if the cruciate mechanism is damaged or absent.
\end{abstract}

Case Presentation: We present the cases of two patients, 55 and 67 years old each, one man and one woman operated at least 10 (up to 15) years before for knee gunshot injuries trauma resulting in one total and one subtotal patellectomy. These patients were facing knee pain and arthrosis. One of the patients had severe ankylosis. The other had a very restricted ROM of 30 degrees (10/10/40). $\mathrm{X}$ rays and in one case MRI were performed to clarify the anatomical view. The total knee endoprosthesis with the patellar part was implanted (in the ankylosis case the revision endoprosthesis was implanted per primam). The most difficult point of the operation was the establishing of the right patellar-prosthesis point insertion and the way of insertion.

Outcomes: After the implantation of the knee prosthesis, we tried to find the old place of the patella, although the shortening of the quadriceps muscle has altered the geometry. It was temporarily fixed and after the probe of patellar tracking, it was fixed definitively. The technique of fixation had to be improvised with holes on the lateral side or the patellar part and fixed with ethibond suture nr.5. Discussion: The CPM and the quadriceps strengthening with the usual schema (next 
day start, four hours CPM in a day) was applied. After the first month we had 0/0/60 for both of the patients. We reached $0 / 0 / 90$ for the man with post ankilosis patient and 0/0/70 for the other three months later. After 6 months the post ankilosis patient was pain free with $0 / 0 / 90$ and the woman with $0 / 0 / 70$, complained of pain. The cause of the pain was due to the iatrogenic medialization of the patellar implant .A second operation to performed a quadriceps plasty by folding the lateral aspect of the tendon on the patella insertion was planned.

Keywords: Post-traumatic patellar absence, Knee endoprosthesis

\section{Acute Traumatic Patellar Tendon Rupture}

Edvin SELMANI ${ }^{1}$, Fatmir BRAHIMI ${ }^{1}$, Leard DURAJ ${ }^{1}$, Valbona SELMANI ${ }^{1}$

${ }^{1}$ Orthopedic service at University Hospital of Trauma Tirana, Albania

\section{Abstract}

Background: Acute patellar tendon rupture is easy to diagnose but is still often overlooked. The aim of this study was to assess early and late results of surgical treatment of acute patellar tendon rupture with Krakow technique. Material and Methods: A retrospective study included 4 patients (4 males). Mean age was $29.6 \pm 9.9$ years. Lesions comprised 4 tendon body ruptures, Tendon repair was achieved with Krakow technique. Results were evaluated in 2 steps: on patient files at a mean follow-up of 7.1 months (range, 3-24 months) to assess complications and early functional and radiological results; and by phone at a mean follow-up of 2.3 years in order to assess long-term functional outcome on Lysholm score and patient satisfaction. Results: Four knees were assessed at a mean 7.1 months. Mean knee flexion was $128.5^{\circ} \pm 7.5^{\circ}$ (range, $85^{\circ}-150^{\circ}$ ), extension $-1^{\circ}$ (range, $-15^{\circ}$ to $0^{\circ}$ ) and Caton-

Deschamps index 0.96 (range, 0.57-1.29). All knees were further assessed at a mean
2.3 years. Mean Lysholm score was 93.7 points (range, 61-100). All patients were satisfied or very satisfied with the result. All had returned to their previous job, and sports activities.

Conclusions: Patellar tendon rupture has good prognosis if diagnosis and surgical treatment is early.

Keywords: Acute rupture; Patellar tendon; surgical repair

\section{Ankle Fracture with Syndesmotic Disruption - Effect on the Functional Outcome.}

\author{
Marko SPASOV ${ }^{1 *}$, Ilir HASANI ${ }^{1}$, Oliver \\ ARSOVSKI $^{1}$, Ljupco NIKOLOV ${ }^{1}$, Igor \\ KAFTANDZIEV ${ }^{1}$ \\ ${ }^{1}$ University Clinic of Traumatology, Medical faculty \\ of Skopje, Macedonia
}

\begin{abstract}
Introduction: It is widely accepted that reduction of the syndesmosis and placement of one or more screws across the distal tibia and fibula restores the anatomy and allows the syndesmosis to heal. However, there is no consensus regarding the specifics of screw placement. The aim of the present study was to evaluate the clinical and radiological result in patients with intact, broken and removed syndesmotic screw.
\end{abstract}

Materials and Methods; The study was conducted at our institution in a period of two years, and minimum follow up period of 12 months. Patients who had undergone open reduction and internal fixation of an ankle fracture with screw stabilization of the disrupted syndesmosis were included. Fracture type was defined using DenisWeber classification system. Functional result was measured using AOFAS score and VAS scale was used for pain assessment. The statistical difference between the three groups was tested with non-parametric statistics.

Results; The study population was separated into three groups regarding the state of the syndesmotic screw: intact, 
broken and removed. There was no statistical difference between the demographics of these groups. There was statistically significant difference in AOFAS score among the three tested groups due to difference between intact and broken group. Regarding the VAS scale, the score in intact group was significantly higher compared to broken and removed group.

Conclusion; We found no statistical difference in clinical outcome of patients who had their syndesmotic screw removed and those who did not. However, patients with broken screws fared best of all. Widening of the syndesmosis was not seen after removal or breakage of the screw. Our data do not support the routine removal of the intact or broken syndesmosis screw.

Keywords: ankle, syndesmosis, fracture, surgery, removal

\section{Results of Chronic Achilles Tendon Ruptures Repaired With $V$-Y Tendon Plasty and Fascia Turndown.}

Edvin SELMANI ${ }^{1 *}$, Fatmir BRAHIMI ${ }^{1}$, Leard DURAJ $^{1}$, Valbona SELMANI ${ }^{1}$

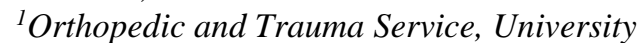
Hospital of Trauma, Tirana, Albania

\section{Abstract \\ Background: This study aimed to evaluate the long-term follow-up results of $\mathrm{V}-\mathrm{Y}$ tendon plasty with fascia turndown, for repairing chronic Achilles tendon ruptures.}

Material and Methods: Seventeen patients (12 males, 5 females), who were diagnosed with chronic Achilles tendon rupture and met the inclusion criteria, were included in the study. These patients received treatment by means of $\mathrm{V}-\mathrm{Y}$ tendon plasty with fascia turndown from January 2005 to December 2011. Clinical outcomes of the patients were assessed by using isokinetic strength testing, questioning the patient regarding residual discomfort, pain, or swelling and having the ability to perform heel rises and using American Orthopaedic Foot \& Ankle Society's (AOFAS's) Ankle-Hind Foot Scale score. Mean follow-up duration was 10 years (7-13 years). Results:Meantime from the injury to operative treatment was 6 months. Mean operative defect of Achilles tendon in neutral position after debridement was 5 $\mathrm{cm}$. During the follow-up, the mean calf atrophy was $3.4 \mathrm{~cm}$. The mean 30 degrees/s plantarflex and 120 degrees/s plantarflex peak torques were 89 and 45 $\mathrm{Nm}$, respectively. The mean 30 degrees/s plantarflex peak torque deficiency was $16 \%$. The mean 120 degrees/s plantarflex peak torque deficiency was $17 \%$. The average peak torque deficiency was $17 \%$. The pre- and postoperative mean AOFAS Ankle-Hindfoot Scale scores were 64 and 95 , respectively. No patient had a rerupture. Superficial wound infection was treated with oral antibiotic therapy in 2 patients (11\%).

Conclusions: The V-Y tendon plasty with fascia turn down for repairing chronic Achilles tendon ruptures yielded results comparable with the literature regarding clinical outcomes. This method did not require synthetic materials for augmentation and was an economic alternative compared to other repair methods.

Keyword: Achilles tendon rupture, tendon plasty, fascia turndown...

\section{Meniscal Repair of Bucket Handle Meniscus Tears - Accelerated or Traditional Rehabilitation}

\author{
Alan ANDONOVSKI ${ }^{1 *}$, Ilir HASANI ${ }^{1}$, \\ ANDONOVSKA B ${ }^{1}$, Rezeart DALIPI ${ }^{1}$. \\ ${ }^{1}$ University Clinic for Traumatology, Orthopedic \\ Surgery, Anesthesiology and Intensive Care. \\ Faculty of Medicine, University "Ss Cyril and \\ Methodius” Skopje, Macedonia.
}

\section{Abstract}


Introduction: Post-operative weight bearing and knee range of motion after meniscal repair is still debatable among physicians. There is a lack of consensus regarding the optimal postoperative rehabilitation protocol following meniscal repair.

Purpose: The aim of our study was to compare the clinical results in patients with bucket-handle meniscal repair who were submitted to an accelerated rehabilitation protocol that included immediate full range of motion and weightbearing after the surgery and those who were submitted to a traditional rehabilitation protocol with restricted range of motion and weightbearing.

Methods: Patients who underwent meniscal repair of buckethandle meniscal tears using the outside-in and inside-out technique were included in our study and divided into two groups:

Group I where an accelerated rehabilitation protocol that included immediate knee full range of motion and weightbearing was performed and group II where a traditional rehabilitation protocol with restricted range of motion and weightbearing was conducted. The patients were evaluated postoperatively after an average of 8 months (range 6-11 months) by clinical examination, Tegner and Lysholm scores, measuring sagittal knee stability with Rolimeter, proprioception evaluation and MRI imaging.

Results: Twenty-eight patients were included in our study. Overall, $72 \%$ of patients from group I and $84 \%$ of patients from group II reported clinically good results. Clinical examination and MRI imaging showed meniscal retear in 2 patients only from group I. There was not a significant difference between the groups regarding the Lysholm (88 vs 80 , respectively; $p=0.3$ ) and Tegner ( 7.5 vs 7 , respectively; $p=0.97)$ scores. The results from the measured sagittal knee stability and proprioception also did not show significant differences between the groups.
Conclusion: Our study showed that although the increased rate of meniscal retears an accelerated rehabilitation protocol can obtain comparable clinical results as traditional rehabilitation protocol in patients with bucket handle meniscal repairs. Individual approach and good coordination with the referent orthopedic surgeon should be obtained to avoid the increased rate of meniscal retears.

Keywords: Bucket-handle meniscal repair, Accelerated or Traditional rehabilitation protocol, Clinical results.

\section{Osteochondral Transplantation Technique Pitfalls in Knee Osteochondral Defects (MOSAICPLASTY/OATS technique)}

\author{
Ledian FEZOLLARI ${ }^{1}$, Leard DURAJ ${ }^{1}$, Ilir \\ HASMUCA $^{1}$, Dorjan HABILI ${ }^{1}$, Alban \\ HYSENAJ $^{1}$, Arnel MICI, A DIZDARI ${ }^{1}$, Ylber \\ ZAMA $^{1}$, Gjergji CAUSHI ${ }^{1}$, Vilson RUCI ${ }^{1}$, Artid \\ DUNI ${ }^{1}$, Ergys CAMI ${ }^{1}$, Korab SEJDINI ${ }^{1}$, Serdi \\ MEMINI ${ }^{1}$ \\ ${ }^{l}$ Ortopedic Service University Hospital of Trauma, \\ Tirana, Albania
}
Abstract
The MOSAICPLASTY technique involves obtaining small osteochondral cylindrical grafts from the less weight-bearing periphery of the femur at the patellofemoral joint, and transporting them to the prepared defect site. With a combination of $2.7 \mathrm{~mm}, 3.5 \mathrm{~mm}, 4.5$ $\mathrm{mm}, 6.5 \mathrm{~mm}$, and $8.5 \mathrm{~mm}$ grafts, the site is filled with 70-90\% transplanted hyaline cartilage. Fibrocartilage "grouting" growing upward from the prepared cancellous bed will complete the MOSAICPLASTY graft. Placing the grafts perpendicular to the surface, at the level of the original articular surface, is paramount to the success of the operation. The best candidate for osteochondral autologous transplantation (OATS) is the young (20-30 years), thin ( $<25$ BMI) patient who suffers a symptomatic, traumatic, unipolar, small $(<2 \mathrm{~cm} 2)$, and type IV outerbridge chondral defect exposing subchondral bone, confirmed previously with 
an MRI. Cartilage transplantation can be performed either arthroscopically or open, including the miniopen technique. Some surgeons advise that those unfamiliar with the procedure should begin with the all open technique and after several cases, move to an arthroscopic procedure. This article aims to discuss osteochondral transplantation technique pitfalls in knee osteochondral defects and to give a review of literature.

Keywords: MOSAICPLASTY / OATS technique, knee osteochondral defects, pitfalls

\section{A combination of French and Ponseti method as treatment for pedes equinovarus congenitalis - Case report}

Anila BELCHISHTA ${ }^{1}$, Anastasika POPOSKA ${ }^{1}$, Daniela GEORGIEVA $^{1}$

${ }^{1}$ University, University Clinic for Orthopedic SurgerySkopje.

Abstract

A case of a 2 weeks old female baby with pes equinovarus congenitalis bothsides was brought to our Orthopaedic Clinic. It's been detected a breech presentation as a fetus.

We started the treatment with the French method, a conservative method with daily physical therapy for the first two weeks, with daily manipulations of the feet along with stretching of the feet, followed by taping in order to maintain the range of motion gains achieved at the end of each sesion. Another focus was strengthening the peroneal muscles which is thought to contribute towards long-term correction. Afterwards physical therapy sessions were weaned down to once a week followed by casting, until the child reached six months old. Ponseti second method was the following performed, a percutaneous heel cord release or tenotomy of Achilles tendon and capsulotomy with sedation and monitored anesthesia care. A small dressing is applied and a final clubfoot cast is applied with the foot in a fully corrected position. This cast was left in place for 8 weeks, replaced every 2 weeks.

After correction was achieved with casting, maintenance of correction started with full-time (23 hours per day) use of a brace — also known as a foot abduction brace (FAB) - on both feet, Denis-Browne bar, for 3 months. After 3 months, brace wear was decreased and used mostly when sleeping for naps and at night-time. This part-time bracing is recommended until the child is 4 years of age.

Keywords: Pes equinovarus, French method, Ponseti II method, Breech presentation

\section{Complex Abdominal Wall Reconstruction: Practical Approaches}

Rifat LATIFI ${ }^{1}$

${ }^{I}$ The Felicien Steichen Professor and Chairman of Surgery

New York Medical College, School of Medicine Director, Department of Surgery, Westchester Health Network

Department of Surgery, New York Medical College

\section{Abstract}

With advances in abdominal surgery and the use of Damage Control Surgery (DCS) in the management of major trauma, and patients with abdominal catastrophes, complex abdominal wall defects have become the new surgical disease, and the need for complex abdominal wall reconstruction (CAWR) has increased dramatically. Subsequently, how to reconstruct these large defects has become a new surgical question. While most surgeons use native tissue, whenever possible, evidence suggests that synthetic or biologic mesh needs to be added to large ventral hernia repairs for optimal long-term outcome. One particular group of patients who exemplify "complex" are those with contaminated wounds, enterocutaneous fistulas, enteroatmospheric fistulas, and/or stoma(s), where synthetic mesh is to be avoided if at all possible.

While DCS and open abdomen management (OAM) in trauma and critically ill patients or those who have survived initial catastrophic abdominal event, has been shown to be lifesaving, closing the abdomen and regaining abdominal wall function to avoid severe 
consequences of DCS and OAM has

become modus operando in our

department. I will review our most recent experience in CAWR in the Acute Phase of

Injury or intra-abdominal catastrophe, using biologic mesh. Furthermore, most recently, biologic mesh has become the new standard in high-risk patients with contaminated and dirty infected wounds. While biologic mesh is the most common tissue engineered used in this field of surgery, level I evidence is needed on its indication and long-term outcomes.

Various techniques for reconstructing the abdominal wall have been described, however the long-term outcomes for most of these studies, are rarely reported. In this presentation, I will outline current practical approaches to perioperative management and definitive abdominal reconstruction in patients with complex abdominal wall defects, with or without fistulas, as well as those who have lost abdominal domain and review our experience in almost 300 patients. In our most recent study (unpublished data) we have shown that frailty appears to be a more important predictor of adverse outcomes after CAWR with BM than age. In addition to frailty, other significant independent predictors of outcomes include open abdomen, congestive heart failure, transplant and alcohol use. All future studies should assess frailty as a potential predictor of outcomes.

\section{Role and Control of Infections and Biofilms in Surgical Wounds}

\section{Jasenka ŠKRLIN ${ }^{1}$}

${ }^{1}$ Department of Clinical Microbiology and Hospital Infection, Committee for Infection Control, Dubrava University Hospital - Zagreb, CROATIA. burden, associated with increased morbidity, mortality and healthcare costs; $80 \%$ of SSIs may involve a microbial biofilm.

Microorganisms routinely contaminate, colonize, and often infect wounds of all types. Wound infection and possibly highlevel colonization appear to be major barriers to healing. The presence and negative effects of biofilm in chronic wounds has been increasingly recognised. Recent studies suggest that biofilmproducing organisms play a significant role in persistent skin and soft tissue wound infections in the postoperative surgical patient population. Biofilm, on an organizational level, allows bacteria to survive intrinsic and extrinsic defenses that would inactivate the dispersed (planktonic) bacteria. SSIs associated with biomedical implants are notoriously difficult to eradicate using antibiotic regimens that would typically be effective against the same bacteria growing under planktonic conditions. This biofilm-mediated phenomenon is characterized as antimicrobial recalcitrance, which is associated with the survival of a subset of cells including "persister" cells.

An efficient treatment of biofilm infections needs a well-established multidisciplinary collaboration, which includes removal of the infected foreign bodies, selection of biofilm-active, sensitive and wellpenetrating antibiotics, systemic or topical antibiotic administration in high dosage and combinations, and administration of anti-quorum sensing or biofilm dispersal agents.

\section{Advances in Complicated Intra-Abdominal Infections Management.}

Boris E. SAKAKUSHEV ${ }^{1}$

${ }^{1}$ Medical University Plovdiv Chair of Propaedeutic of Surgical Diseases, First Clinic of General Surgery University Hospital St George Plovdiv, Plovdiv, Bulgaria

\begin{abstract}
Despite remarkable developments in the advancements in the operating room, and implementation of bundles, surgical site infections (SSIs) remain a substantial
\end{abstract}


Abstract

Sepsis is world problem requiring global geographical answer in all engaged sectors. CIAI are common diseases with considerable morbidity and mortality, despite progress in management in the last decades. Key issues in the management of AS are early diagnosis and patient resuscitation, timely and adequate control of the source of infection and antibiotic treatment.

This is a review of the latest evidencebased recommendations on the critical issues constituting ES strategy for treatment of abdominal sepsis, focusing on the crucial component - liquidating the source of infection. The fundamental principles, timing and most common techniques are envisaged in the light of the most recent world guidelines on the problem from the last 3 years.

Future tendenciesof source control focus on precise risk assessment for operationof patients with CIAI, wider implementation of non-operative techniques/percutaneous drainage/, more organ-saving, limited/physiological/ operations if possible.

All these will assure improved patients' safety, shorter hospitalization and expenses, lower disability rate and better QOL.

Keywords:Emergency Surgery, Abdominal Sepsis, Complicated IntraAbdominal Infections

\section{Management of Abdominal Compartment Syndrome in Non traumatic Patients.}

Nuhi ARSLANI ${ }^{*}$

${ }^{1}$ University Clinical Center Maribor, Department for Abdominal Surgery, University of Maribor, Faculty of Medicine, Slovenia

\author{
Abstract \\ Introduction; Increased intra-abdominal \\ pressure is called intra-abdominal \\ hypertension (IAH). Abdominal \\ compartment syndrome (ACS) refers to
}

organ dysfunction caused by intraabdominal hypertension. Intra-abdominal pressure is defined as the pressure created within the abdominal cavity the normal. IAP for critically ill adults is $5-7 \mathrm{mmHg}$. Intra-abdominal hypertension is a sustained or repeated IAP $>$ than $12 \mathrm{mmHg}$ ( figure 2).

There are various grades of IAH, Grade 1 IAP $12-15 \mathrm{mmHg}$, Grade 2 IAP 16$20 \mathrm{mmHg}$, Grade 3 IAP $21-25 \mathrm{mmHg}$ and grade 4 an IAP $>25 \mathrm{mmHg}$.

Patients in the intensive care unit (ICU) are at risk of developing of intra abdominal hypertension (IAH) and abdominal compartment syndrome (ACS).

ACS can be classified as primary or secondary .

Primary ACS is due to injury or disease in the abdominopelvic region (eg, abdominal trauma, hemoperitoneum, pancreatitis); intervention (surgical or radiologic) of the primary condition is often needed.

Secondary ACS refers to conditions that do not originate in the abdomen or pelvis (eg, fluid resuscitation, sepsis, burns) ACS can impair the function of nearly every organ system. Physiologic consequences include impaired cardiac function, decreased venous return, hypoxemia, hypercarbia, renal impairment, diminished gut perfusion, and elevated intracranial pressure.

Diagnosis of ACS requires that intraabdominal pressure be measured.

Following surgical decompression, an open abdomen is maintained using a variety of temporary abdominal closure techniques .

Keywords:Intra-abdominal pressure, Intraabdominal hypertension, Abdominal compartment syndrome, Abdominal perfusion pressure 


\section{Acute Mesenteric Ischemia, Diagnostic and Surgical Challenges}

Rovena BODE ${ }^{1}$, Etmont ÇELIKU ${ }^{1}$, Xheladin DRAÇINI ${ }^{1}$, Henri KOLANI ${ }^{1}$, Adrian NELAJ ${ }^{1}$ ${ }^{I}$ Department of General Surgery, Mother Theresa Hospital Tirana

\section{Abstract}

In all acute surgical admissions, the incidence of AMI is estimated at 0.09$0.2 \%$. Therefore, diligence is always required because if untreated mortality has been reported in the range of 50-70\%. Early diagnoses and timely surgical intervention are the cornerstones of modern treatment and are essential to reduce the high mortality of this entity. Endovascular approaches in parallel with modern imaging techniques may provide new options, but what are the outcomes of surgery in patients with overt peritonitis signs, where prompt laparotomy is mandatory? Damage control surgery is an important option in the AMI patient and operative management requires adequate excision of clearly necrotic bowel and a high index of suspicion of borderline segments. What about second look techniques outcomes? Their timing....and decisions regarding stomas and anastomosis... What about the open abdomen techniques indications? What about our experience, our outcomes? The aim of this study is to give a glance at our experience in this high-grade mortality entity.

A retrospective analysis was performed on patients who were diagnosed with acute intestinal ischemia between june 2017 and july 2018. Patients demographics, clinical characteristics, risk factors, surgical procedures and survival were analysed.

Key words: acute mesenteric ischemia(AMI), damage control

surgery(DCS), endovascular revascularisation

\section{Gastroduodenojejunal Intussuspection Due to Peutz- Jeghers Syndrome. A Case Report}

Shkelzen OSMANAJ*, Agron DOGJANI. Naim DIZDARI. Elona MARKECI. Astrit MUSTAFA. Vidi DEMKO

University Hospital of Trauma, Tirana ALBANIA

\section{Abstract}

Peutz-Jeghers syndrome (PJS) is a rare autosomal dominant disorder occurring in 1 in 150000 people. It is characterized by familial mucocutaneous pigmentation (dark freckles on face, lips, buccal mucosa, palm and soles)

and hamartomatous polyps in the gastrointestinal tract.

Gastroduodenojejunal intussusception is the rarest forms of adult intussusception. An 27 years- old Albanian male from Kavaja was admitted to our hospital with abdominal distension, recurrent abdominal pain and intermittent non-bilious vomiting of about 6 months standing. Endoscopy revealed two huge cauliflower- like polyps and three small polyps in the stomach, and the biopsy specimens showed hyperplastic polyps. Moreover, multiple pigmentation of the lyps or buccal mucosa and in perinasal skin. The patient had been mild anemia due to internal bleeding. In ct abdominal with contrast was seen intussusception. We made open surgery to resolve the case.

Keywords; peutz-jeghers syndrome, gastro-duodeno-jejunal intussusception, gastric-outlet obstruction and mucocutaneous pigmentation.

\section{Surgical treatment of a Ruptured Pancreaticoduodenal Artery Aneurysm: Case Report and Review of the Literature}

Henri KOLANI ${ }^{1}$, Ejona CELIKU ${ }^{3}$, Earta GEGA ${ }^{4}$, Etmont CELIKU ${ }^{1,2}$

${ }^{1}$ Surgeon at First Clinic of Surgery- University Hospital Center "Mother Teresa" Tirana, ALBANIA 
${ }^{2}$ Chief of Surgery Department and Chief Surgeon at First Clinic of Surgery- University Hospital Center "Mother Teresa" Tirana, ALBANIA

${ }^{3}$ AnatomoPathologist Doctor at University Hospital Center "Mother Teresa" Tirana, ALBANIA

${ }^{4}$ Student of General Medicine at University of Medicine of Tirana, ALBANIA

\begin{abstract}
Introduction: Pancreaticoduodenal artery (PDA) aneurysm associated with celiac artery (CA) occlusion or stenosis is extremely rare and occurs in $2 \%$ of all visceral aneurysms.

Method: We present the case of a 58-yearold female diagnosed with ruptured pancreaticoduodenal aneurysm. The bibliography used was taken from PubMed.
\end{abstract}

Summary: We report the case of a 58-yearold woman who presented at the Surgical Emergency Unit with acute abdominal pain radiating at the back, fatigue, discomfort with a history of a minor trauma. Physical examination is relevant for tenderness of the whole abdomen on palpation. Workup reveals anemia with $\mathrm{Hgb}$ at $8.3 \mathrm{~g} / \mathrm{dL}$ and $\mathrm{RBC}$ count at $2.8 \times 10^{6} / \mathrm{mm}^{3}$. Imaging studies show retroperitoneal hematoma, contrast accumulation near the pancreaticoduodenal arcade but with no evident relation to the superior mesenteric artery, peripancreatic, perihepatic and free peritoneal fluid. The patient was successfully treated with surgery and was discharged on the $15^{\text {th }}$ post-operative day. Conclusion: Rupture of visceral artery aneurysms, although rare, may be a relevant differential diagnosis of an acute abdomen. When the patient comes to the emergency department with abdominal pain, a diagnosis of visceral artery aneurysm rupture should be considered.

\section{Hantavirus Infection Causative Agent of Acute Renal Injury}

Lutfi ZYLBEARI ${ }^{1}$, Kastriot HAXHIREXHA ${ }^{1}$, Agron DOGJANI ${ }^{2}$, Sadi BEXHETI ${ }^{1}$, Ferizat DIKA-HAXHIREXHA ${ }^{1}$, Aulona HAXHIREXHA ${ }^{3}$, Zamira BEXHETI ${ }^{1}$, Gazmend ZYLBEARI ${ }^{1}$
${ }^{I}$ Faculty of Medical Sciences, University of Tetovo

- Tetovo, R. of Macedonia

${ }^{2}$ University Hospital of Trauma, Tirana, Albania

${ }^{3}$ University of Medicine, Tirana, Albania

\section{Abstract}

Acute kidney injury (AKI) is a rapid condition occurring within a few hours to a few days. Early detection of Hantan virus (HV) induced AKI is the golden standard for management and treatment of the AKI, focused on reduction and lowering of the further kidney injury. Adequate hydration should begin immediately after diagnosis of AKI, thus maintaining: haemodynamic equilibrium of the kidneys, renal perfusion, correct and equilibrated electrolytes, acidbase equilibrium, overall kidney homeostasis, proper kidney oxygenation and prevention of drug induced nephrotoxicity, and maintenance of renal function.

Materials and methods: In our scientific research we report on six patients suffering from $\mathrm{AKI}$ as a consequence of $\mathrm{HV}$ infection, treated in the Department of Internal Medicine, at the Clinical Hospital in Tetovo, and the University Nephrology Clinics in Skopje, during a 5 years period (2006-2010). The mean ages of the patients were 35.5 years old ( \pm 4.6 years) for the 4 male patients, and the 2 female patients were aged 33 years old $( \pm 2.6$ years).

Results: A percutaneous renal biopsy was performed to all six patients, and immunofluorescence microscopic examination afterwards. The results confirmed the acute interstitial nephritis diagnosis for four patients, whereas for two patients the diagnosis of acute proliferative glomerulonephritis. Luckily the progress of the disease ended with complete recovery for all the patients...

Conclusion: We conclude that early detection of HV infection, full assessment of the clinical state, early diagnosis of AKI, immediate onset of treatment (by following the protocol for AKI treatment) at the Intensive Care and Reanimation Unit, remain the keystone for proper and successful treatment, management, and prevention of exacerbation of AKI. 
Key words: Hantavirus, Acute Kidney Injury

\section{Oral and Maxillofacial Infections as Differential Diagnosis in Fever of Unknown Origin.}

Ermira $\mathrm{MUCO}^{1}$, Neada HOXHA ${ }^{1}$, Arta KUSHI ${ }^{1}$, Jonida MEHMETI ${ }^{3}$, Arlind MYFTARI ${ }^{2}$.

${ }^{1}$ The Infectious Disease Service, UHC "Mother Tereza" Tirana. ALBANIA.

${ }^{2}$ Oral and Maxillofacial Surgery. UHC "Mother Tereza" Tirana. ALBANIA

${ }^{3}$ Infectious Disease Service, Hospital of Saranda. ALBANIA

\begin{abstract}
Fever of unknown origin (FUO), refers to a condition in which the patient has an elevated fever but despite investigations by a physician no explanation has been found. In 1961 Petersdorf and Beeson suggested the fever of unknown origin criteria: fever higher than $38.3^{\circ} \mathrm{C}\left(101^{\circ} \mathrm{F}\right)$ on several occasions; persisting without diagnosis for at least 3 weeks and at least 1 week's investigation in hospital.

The spectrum of differentials includes infections, neoplasms, inflammatory diseases, miscellaneous disorders and undiagnosed conditions.

In the context of infections, dental disease is one potential cause of persistent fever. Therefore, doctors of infectious disease often require the help of their colleagues of oral and maxillofacial surgery. Their intervention and our treatment with the proper antibiotics bring about the best solution to the situation. So this collaboration is a necessity.
\end{abstract}

Keywords: dental infections, fever of unknown origin, differential diagnosis

177. Severe Tricuspid Valve Endocarditis in a Hemodialysis Patient. Clinical Presentation and Outcome.
Ermira MUCO ${ }^{1}$, Neada Hoxha ${ }^{1}$, Arta KUSHI $^{1}$, Jonida MEHMETI ${ }^{1}$, Vilma CADRI ${ }^{3}$, Albana $\mathrm{DOKO}^{2}$

${ }^{1}$ Service of Infectious Diseases, University Hospital Center "Mother Tereza", Tirana, ALBANIA.

${ }^{2}$ Service of Cardiology, University Hospital Center

"Mother Tereza", Tirana, ALBANIA.

${ }^{3}$ Service of Nefrology, University Hospital Center

"Mother Tereza”, Tirana, ALBANIA.

\begin{abstract}
Background: Infection is the most common cause of death in hemodialysis patients, after cardiovascular disease. Infective endocarditis is a potentially lethal complication.

Aim: Our aim was to report clinical and echocardiographic characteristics, microbiological profile, management of hemodialysis patient who presented with fever of unknown origin and resulted infective endocarditis.
\end{abstract}

Results: We present the case of a 38 yearsold woman with 27-days history of high fever $39^{\circ} \mathrm{C}$ comes in the emergency of the Service of Infectious Diseases. She was with diabet mellitus for 35 years and hemodialisis patient for three years. She was blind. Transthoracal and transesophageal echocardiography showed one vegetation on the anterior leaflet of the tricuspid valve, $3.5 \mathrm{~cm} 2$. Blood cultures resulted positive for candida spp. Chest computed tomography showed septic pulmonary emboli.

Antibiotic therapy included Vancomycine, Meropenem, Fluconazol and symptomatic treatment. After five weeks of antibiotic therapy, repeat TTE/TEE showed a resolution of vegetations, $0.2 \mathrm{~cm}^{2}$. The patient was released in optimal health condition. The patient is restarted after a month with high fever. Transthoracal and transesophageal echocardiography showed increased vegetation, $4 \mathrm{~cm}^{2}$ and the repeated TEEafter a week showed one vegetation, 6 $\mathrm{cm}^{2}$. Because of her health conditions, she did not undergo a cardiac surgery. The situation as it came and it became more difficult. Cardiac and pulmonary problems were ordered. Anemia became worsening 
and deeper, until after three weeks the sick person died.

Conclusion: Infective endocarditis is strongly associated with morbidity and mortality in hemodialysis patient. If patients require valve replacement surgery, mortality is even higher.

Keywords: Infective endocarditis, hemodialisis patient, blood culture.

\section{Trauma and Management of Infectious Disease.}

Petrit BIBERAJ $^{1 *}$, Shqipe LUTA ${ }^{1}$, Albana KRECA $^{1}$

${ }^{I}$ Faculty of Technical Medical Sciences, University of Elbasan "A. Xhuvani", Elbasan, Albania

\footnotetext{
Abstract

Accidents and trauma are one of the world's mostserious but neglected health problem. The fast movingtransportation systems, unprecedented and unplannedurbanisation and changing social patterns have contributedto the global increase in the incidence of trauma to human body.

Traffic accidents are an endemic disease which affects mainly the young adults in the economically productive agegroups and are the leading cause of death in persons under 44 years of age. Globally, $26 \%$ of all deaths in the agegroup of 15-44 years in 2015 were due to injuries. Infections in Traumatised Patients Accidents or violence can result in penetrating trauma. Contaminated penetrating foreign bodies introduced at the time of wounding cause infection, especially high velocity projectiles, which result in cavitation. Surgical debridement reduces potential infection; however, perioperative antibiotics are usually indicated owing to studies demonstrating high rates of sepsis in the pre-antibiotic era. Open injuries have a potential for serious bacterial wound infections, including gas gangrene and tetanus, and these in turn may lead to long term disabilities, chronic wound or bone
}

infection, and death. Wound infection is particularly of concern when injured patients present late for definitive care or in disasters where large numbers of injured survivors exceed available trauma care capacity. Trauma-associated pathogens include Gram-positive, Gram-negative and anaerobic pathogens. Antibiotic resistance is increasing, and several recent panels have sought to develop guidelines for perioperative prevention and empiric treatment of infection to limit usage and reduce selective pressure for resistance The interruption of tissue integrity, hemorrhage and tissue hypoperfusion, frequency of invasive procedures, and impaired host defense mechanisms all have a major impact on subsequent infection.It is well known that trauma patients with hospital-acquired infections (HAIs) are at increased risk for mortality, have longer length of stay, and incur higher inpatient costs.

\section{Cost-effectiveness of Thyroid Fine Needle Aspiration Cytology for Diagnosis and Treatment of Thyroid Malignant Pathologies in Pediatric Age}

\author{
Ilir ALIMEHMETI ${ }^{1,2,3^{*}}$, Myriam HUNINK ${ }^{3,4}$ \\ ${ }^{1}$ Projects and International Affairs Unit, Faculty \\ of Medicine, University of Medicine, Tirana, \\ Albania \\ ${ }^{2}$ Department of Family and Occupational \\ Health, Faculty of Medicine, University of \\ Medicine, Tirana, Albania \\ ${ }^{3}$ Department of Epidemiology, Erasmus \\ University Medical Center, Rotterdam, the \\ Netherlands \\ ${ }^{4}$ Department of Health Policy and Management, \\ Harvard School of Public Health, Harvard \\ University, Boston, MA.USA.
}

\begin{abstract}
Background: Thyroid nodules are rare during pediatric age, affecting $1.7 \%$ of children and adolescents, but the high malignancy prevalence at presentation has
\end{abstract}


warranted an aggressive clinical approach. The most frequent surgery technique is total thyroidectomy, which is followed by short-term and long-term complications. Fine needle aspiration (FNA) cytology is the most accurate tool in the diagnosis of a thyroid nodule. However, there are contradictions if it should be used as a first-line strategy in the pediatric age. Thus, our aim was to analyze costeffectiveness of surgery, FNA and observation of thyroid malignant pathologies in pediatric age.

Material and Methods: A decision model was created to trade-off between three different strategies:

- Surgery for all nodules, regardless of their malignant or benign nature;

- Testing through FNA, and then perform surgery according to the FNA result;

- Observation, regardless of their malignant or benign nature.

Cost-effectiveness was calculated in terms of costs and quality-adjusted life years (QALY). All variables were entered as distributions. Willingness to pay was set to $50,000 \$$.

Results: In the cost-effectiveness analysis, surgery was dominated by FNA.

Observation was less effective and cheaper than FNA, which presented an ICER value of 46,948.48.

In the 1-way cost-effectiveness sensitivity analysis for the probability of malignancy from $0 \%$ to $100 \%$, FNA was always superior to the surgery strategy, and the threshold of superiority to the observe strategy was probability of malignancy $=23.5 \%$. In the probabilistic sensitivity analysis, FNA resulted superior to surgery.

Conclusions: FNA is more cost-effective than the surgery strategy in the diagnosis and clinical work-up of thyroid nodules in children.

Keywords: Fine needle aspiration, thyroid nodule, probability of malignancy, Thyroid Surgery.

\section{Hairy Cell Leukemia After Sleenectomy with Ascitis In the Time of The Diagnosis. A Case Reports.}

Eriselda TAULLA ${ }^{1}$,_Bajram BEGAJ ${ }^{1}$, Ilir KAJO ${ }^{1}$, Elvina SHABA ${ }^{1}$

${ }^{1}$ Department of Internal Medicine, University

Hospital of Trauma, Tirana, ALBANIA.

\begin{abstract}
Introduction: Hairy cell leukemia (HCL) is a unique chronic lymphoproliferative disorder characterized by cytoplasmic villous (hairy) projections and diffuse infiltration of the bone marrow and spleen, leading to peripheral cytopenia and splenomegaly.

The aim: How to differentiate a HCL. $\mathrm{HCL}$ is a rare chronic leukemia (B-cell malignancy). It consistutes $2 \%$ of all leukemias
\end{abstract}

Case description: A 45-year-old man was hospitalized with a three months history of abdominal swelling, dyspnea, weakness, anorexia, the physical examination showed cutaneous and conjuctivalpallor, ecchymosis in the right hipochondria, the abdomen above thorax level without collateral circulation and hepatomegaly. In 2011 he had done splenectomy and was treated with prednisone. (In that time, he went to the hospital not in Abania with pancytopenia and massive splenomegaly.) CBC showed: bicytopenia (anemia and thrombocytopenia). Serum B12, folates, A.D. A in serum, $\gamma$ interferon, ceruloplasmin, serum copper, ASMA, ANA, AMA, CEA, AFP, CA 19-9, LDH, $\mathrm{RCP}$, total bilirubin $0,7 \mathrm{mg} / \mathrm{dl}$, AST, ALT and other parameters within the normal range.total proteins 4,5 g/dl, TP $60 \%$, INR 1.32 , sideremi $222.21 \mu \mathrm{g} / \mathrm{dl}$.

The bone marrow biopsy shows: hypercellularity, reduction of myeloid and megakariocytar series. IHC: CD20+++, CD3---, CD5+--, CD8+--, CD15+--, CD38+--, CD138+--, Glycophorin A++-, Bcl-2 +++, CD34---, CD117---, MPO---, CD4--- 
Leukocyte Immunophenotyping of peripheric blood: CD19+83\%, CD20+90\%, CD11c+90\%, CD25+90\%, FMC7+60\%, CD103+90\%.

We treat him with Cladribine $0.14 \mathrm{mg} / \mathrm{kg} / \mathrm{d}$ I.V day1-5. The patient one year after admission is in remission.

Conclusion: the diagnosis was Hairy Cell Leukemia

Keyword: Hairy cell leukemia (HCL), spleenectomy, cytopenia, ascitis...

\section{Dyspepsia in Children}

Ferizat DIKA - HAXHIREXHA ${ }^{1,2}$, Sevdije $\mathrm{KOXHA}^{1}$, Ledia $\mathrm{KACI}^{1}$, Aulona HAXHIREXHA ${ }^{2}$

${ }^{1}$ Medical Faculty - Tetove, Macedonia

${ }^{2}$ PHO 'Alba-Med”- Diber, Macedonia

\section{Abstract}

Introduction: Dyspepsia is one of the most common complaint in children.

Disturbances such as upper abdominal pain and fullness, early satiety, bloating, paleness, nausea, or vomiting are the characteristic signs of dyspepsia.

Objectives: the aim of this study is to show our experience in treating children with dyspepsia

Methods: in this study are included 58 children aged between for to fourteen years with signs of dyspepsia. At the admission laboratory investigation were carried out for each patient. Additionally, according to the severity of clinical manifestations, some diagnostic investigations such as abdominal ultrasonography, gastroscopy or microbiological test was performed too.

Results: from 58 children included in this study 35 of them were females and 23 males. In most of the children characteristic symptom were epigastric pain, associated with nausea and vomiting, while in children with moderate form of dyspepsia were present signs such as paleness and bloating. In eight children with the severe recurrent abdominal pain gastroscopy is performed but no any sign of organic disease is registered. In nineteen children coproculture is performed because of suspicion for intestinal parasits but only two of them result positive. Fourteen children with recurrent abdominal pain was found to be Helicobacter pylori positive. Children with mild dyspepsia were initially treated empirically with instructions for environment improvement and diet modification, while in those with severe form empirical treatment associated with acid reducing drugs sometimes combined with antibiotics was the treatment of choice.

Conclusions: dyspepsia is a common disorder among children of all ages. Abdominal pain, nausea, vomiting, bloating and postprandial fullness are characteristic signs of dyspepsia. Empirical treatment associated with acid reducing drugs remains the treatment of choice.

Key words: dyspepsia, recurrent abdominal pain, children

\section{The characteristics and clinical outcome of $P E$ in patients with malignancy.}

Dhimitraq ARGJIRI ${ }^{1}$, Silvana BALA ${ }^{1}$, Fadil GRADICA ${ }^{2}$, Loreta AGOLLI ${ }^{1}$, Arjan MEZINI ${ }^{1}$, Viola SELMANI ${ }^{1}$, Vasil MIHA ${ }^{1}$, Ornela NUREDINI ${ }^{1}$, Jonida TULA ${ }^{1}$

${ }^{1}$ Pneumology Service, University Hospital "Shefqet Ndroqi" Tirana, ALBANIA.

${ }^{2}$ Thoracic and visceral Surgery Service, University Hospital "Shefqet Ndroqi" Tirana, Albania.

\section{Abstract \\ Introduction: Cancer is a well-known cause of venous thromboembolism (VTE). The overall incidence rates of pulmonary embolism (PE) in cancer patients are higher than those in patients with other types of illness and increase rapidly over time.}

Aim: We explored the characteristics and clinical outcome of PE in patients with malignancy.

Material and methods: 105 consecutive PE patients presenting to our tertiary level care have been separated in two groups: G1 group of $87 \mathrm{PE}$ patients without malignancy and G2 group of 18 PE cases 
with malignancy. Diagnostic tolls: Pretest PE probability, Wells score, D-Dimmers, Thoracic CT- scan contrast enhanced. Variable of interest were; Chemotherapy, Site of malignancy, Khorana score, Biomarkers: white cell count $>11.000$, PLT $>350.000, \mathrm{Hb}<10 \mathrm{~g} \%$, and Anticoagulant therapy

Results: of 18 patients enrolled in the study eleven (110 were male and seven (7) female. Average age was 63.5 \pm 9.9 . According to the location of malignancy most of them were lung carcinoma and metastasis $(22.2 \%)$, followed by gastrointestinal and urogenitary cancer $(16 \%)$, lymph proliferative caners with $11 \%$. Over fifty percent $(55.5 \%)$ of patients had intermediate risk for pulmonary embolism according to Khorana risk score and $44 . \%$ to high risk. About $1 / 3$ of the patients (38\%) develop PE during chemotherapy and $62 \%$ of patients after chemotherapy. In $56 \%$ of patients G1 group the clot was found in main artery comparing $20.8 \%$ in $\mathrm{G} 2$ group $(\mathrm{p}=0.0206)$. D-Dimmers in $\mathrm{G} 1$ group was significant higher than $\mathrm{G} 2$ group (4269.9 $\pm 230.8 \mathrm{ng} / \mathrm{l}$ and $2578.1 \pm$ 377.2 respectively) $\mathrm{p}=0.0001$

Conclusions: Risk factors for VTE in the setting of cancer have been well

characterized: Increased age (> 75), solid tumors, metastasis, and time of cancer diagnosis, chemotherapy, and thrombocytopenia. Carefully identify patients at highest risk of VTE and VTE prophylaxis. Guidelines and landmark trials support administration of LMWH in at risk patients.

Keyword: Low molecular weight heparin, venous thromb-embolism, pulmonary embolism

\section{The Relationship Between Trauma and Tumors}

Leart BERDICA $^{1,2,3^{*}}$, Teona BUSHATI ${ }^{1,2,3}$

${ }^{1}$ Department of Anatomic Pathology and Forensic Medicine, Faculty of Medicine, University of Medicine, Tirana, Albania
2 Service of Anatomic Pathology, University Hospital Centre, "Mather Theresa” Tirana, Albania

${ }^{2}$ Dep. of Pathology of American Hospital of Tirana, Albania

\begin{abstract}
Introduction: Trauma by medical definition is "an injury to living tissue caused by an extrinsic agent".(1)

Generally, we are used to be focused in the direct damage of the tissues, surgeons are focused in cleaning the wound to facilitate tissue repair. But sometimes the process of healing doesn't go in the desired direction. In these cases, the surgeons and pathologists help each other to find the reason of the aberrant repair or in discovering tumors during the medical exams.
\end{abstract}

Methods: Pathology results are obtained from several cases from the Skin, Central and Peripheral Nervous, Chest and Thoracic cavity, Abdominal wall and cavity, Testicles, Bone biopsies.

Results: Patients having history of minor, moderate or severe trauma had pathologic exam for different pathology conditions. There is patient that refer mini trauma with the comb of skin head which resulted in hemorrhage and then it was discovered skin cancer or even Melanoma.

There are patients having cranial trauma that during a CT scan has been discovered arterio-venous malformations or various tumors of the brain or leptomeninges. Severe trauma of thoracic wall can produce tumor-like masses in the lung that can be hard to distinguish from a tumor especially when there a lot of time passed between the trauma and diagnostic moment.Trauma of abdominal cavity may reveal several tumors of parenchymatic organs or intestine, or even from the abdominal wall. There are a number of cases where the testicular tumors are discovered by trauma in a football match.

Moderate trauma of the skeleton can produce pathologic fracture that are hard to be repaired and the biopsy result is a tumor or tumor-like condition. 
Conclusions:The "positive" role of trauma for the patient can be the discovery of a concomitant disease even tumors. There some tumors that can be produced by repeated trauma even when it is of little intensity.

Keywords; tumor-like masses,

Melanoma,testicular tumors, mini trauma

\section{Basics of Cervical Cytology \&Management of Pap Smear Result}

Teona BUSHATI ${ }^{1,2,3 *}$, Leart BERDICA ${ }^{1,2,3}$ ${ }^{1}$ Department of Anatomic Pathology and Forensic Medicine, Faculty of Medicine, University of Medicine, Tirana, Albania

2 Service of Anatomic Pathology, University Hospital Centre, "Mather Theresa" Tirana, Albania

${ }^{2}$ Dep. of Pathology of American Hospital of Tirana, Albania

\section{Abstract}

Worldwide, approximately 500,000 new cases of cervical cancer and 274,000 deaths are attributable to cervical cancer yearly, making cervical cancer the second most common cause of death from cancer in women. Fortunately, the incidence of cervical cancer has decreased by more than $50 \%$ in the past $30+$ years, largely due to the increasing use of cervical cancer screening with cervical cytology. The mainstay of cervical cancer screening for the last $60+$ years has been the Papanicolaou test.

The Papanicolaou test, also known as the Pap test or the Pap smear, was developed in the 1940s by Georgios Papanikolaou. It involves exfoliating cells from the transformation zone of the cervix to enable examination of these cells microscopically for detection of cancerous or precancerous lesions.

When abnormal cells are detected on the Pap test, diagnostic testing in the form of colposcopy is often indicated. This testing may be followed by diagnosis of dysplasia via colposcopic biopsies. Subsequent cervical cancer may be prevented through the diagnosis and treatment of these cervical cancer precursors. Results from cervical cytology specimens are reported according to the 2001 Bethesda System Classification. Management of abnormal cytology is done according to 2012 ASCCP Consensus Guidelines.

\section{Pigmentation Disorder and Dermatology Life Quality}

\author{
Ardiana SINANI ${ }^{1}$, Dorela VASHA ${ }^{2}$, Rovena \\ $\mathrm{LICO}^{3}$ \\ ${ }^{1}$ Military Medical Unit at University Hospital of \\ Trauma, Tirana, Albania \\ ${ }^{2}$ Health Inspectorate, Preventive Medicine at the \\ Ministry of Defense, Tirana, Albania. \\ ${ }^{3}$ Speciality Policlinc No 1, Tirana, Albania
}

\section{Abstract \\ Introduction: Vitiligo is an acquired} chronic depigmenating disease of the skin. It causes loss of pigment on effected areas of the skin or mucosae and is characterised by milk white, nonscaley with distinct margins.

Objective: Description of vitiligo. Different clinical forms, and the most frequent consequences of the disease in the social life of the individual.

Results of treatment of simple cases and complex cases. There is very limited information available in the textbooks on vitiligo. This is up-to-date literature overview of the disease including definition, classification, aetiology, histopathology, prognosis, assessment and treatment There is a current lack of consensus in the definition and methods of assessment of this disorder, which makes it difficult to compare the outcomes of different studies of the same treatment.

Conclusion: Skin diseases are very common, affecting over a quarter of the population. Although usually not lifethreatening, skin diseases have a significant impact on the quality of life of patients and cause considerable psychological distress. Vitiligo, a condition which results in the loss of pigment from 
the skin and the cosmetic disfigurement of this seemingly inconsequential skin disease has a major impact on the quality of life of patients especially for people with darker skin. Finally, the aetiology and pathogenesis of vitiligo remains unclear. It is still not understood what causes the destruction of melanocytes.

Keyword: Vitiligo, chronic depigmenating disease, pernicious anaemia,

\section{6. “BURN-OUT” Syndrome, in Military Hospital Personnel.}

Dorela VASHA ${ }^{1}$, Ardiana SINANI ${ }^{2}$.

${ }^{1}$ Health Inspectorate, Preventive Medicine at the Ministry of Defense, Tirana, Albania.

${ }^{2}$ Military Medical Units at University Hospital of

Trauma, Tirana, Albania.

\section{Abstract}

Introduction: "Burn-out" is a phenomenon characterized by fatigue and frustration, usually to professionals whose work is faced with high level of stress that comes as a result of dedication to a cause or a way of living that does not match expectations of the person. Although this appears to be associated with risk factors derived from professional environment, this problem can be present in every person. The aim is to evaluate the level of "burn-out" in the military hospital personnel.

Method: This is a cross-sectional study conducted through the burnout self administered test to the military medical personnel. The Maslach Burn-out Inventory was used as it is the most commonly used tool to self-assess whether you might be at risk of burn-out through three components: exhaustion, depersonalization and personal achievement. According to the level of burn-out it was made a comparison to the types of clinic (therapeutic, surgical, and emergency) and to the level of responsibility.

Results: The study shows that medical personnel of surgical and emergency clinics have a higher level of burn-out than those of the therapeutics clinics. The head of clinics and doctors have a higher level of burn-out than nurses.

Conclusions: There is a clear need for adaptation and expansion of a conceptual framework for a promising approach for designing interventions to help clinicians in mitigation, which cause anxiety and prevent build the flexibility they need to support themselves in clinical services.

Keyword: fatigue and frustration, exhaustion, depersonalization...

\section{Contemporary Considerations on Pain at Pediatric Age}

Edmond PISTULLI ${ }^{1}$, Arian HAMITI ${ }^{1}$, A. Nurçe ${ }^{1}$, A. Nake ${ }^{1}$, D. Selmani ${ }^{3}$, F. Krasniqi ${ }^{2}$

\section{${ }^{1}$ University of Medicin, Tirana, ALBANIA \\ ${ }^{2}$ University Hospital Centre, Department of Neonatology, Pristina Kosovo \\ ${ }^{3}$ University of Medicine Tetovo, MACEDONIA}

\begin{abstract}
Background: Pain is an important part of the disease; it's the first evident symptom. Recently it is not considered anymore by the researchers and the doctors as a symptom, but as a specific disease, defined as "The Fifth Vital Sign.

Adults are the first persons that benefited from the recent researches in this direction, mainly in the last two decades; meanwhile "uncommonly", the pain is still untreated or insufficiently untreated among the children.
\end{abstract}

The aim of the study is to recognize the physiopathological mechanisms behind the pain and these assessment to pediatric. Materials and Methods: A 7-months study from December 1, 2014 to May 31, 2015, was used to assess the acute pain among 177 children from birth to 13 years old in Obstetrics and Gynecology Hospital, U H C, Pediatric Hospital and Outpatient Services in Tirana.For patients, incapable to verbalize the pain intensity in a reliable way (neonates to $<4$ years old), it is used 
the hetero-evaluation. While patients $\geq 4$ years old and $<6$ years old are assessed using the hereto-evaluation and autoevaluation scale according to the Faces Scale and VAS.

Discussion: According to the study's results, the percentage of postoperative patients with intense pain is $47.62 \%$ and with maximum pain is $52.38 \%$. There are $5.77 \%$ non-postoperative patients with moderate pain, $55.77 \%$ with intense pain and $38.46 \%$ with maximum pain. ${ }^{[6][14]}$ In conclusion, all postoperative patients and $94.23 \%$ of non-postoperative children undergoing painful procedures experienced intense and maximum pain.

Conclusions: Assessment is the first step toward the appropriate postoperative and procedural acute pain management. The correct usage of assessment scales, according to a standard protocol ensures to truthfully measure pain intensity.

Keywords: pain, faces pain scale, pediatric patients

\section{Comparison of clinical results between open and laparoscopic appendectomy in the treatment of complicated pediatric appendicitis.}

Kenan KARAVDIĆ ${ }^{1 *}$, Irnis BIŠČO ${ }^{2}$

${ }^{1 .}$ Clinic for Pediatric Surgery, Clinical Center of University Sarajevo, Bosnia and Herzegovina

${ }^{2}$.Faculty of Medicine, University Sarajevo, BOSNIA AND HERZEGOVINA

\footnotetext{
Abstract

Introduction: Approximately 7-10\% of the total population develops appendicitis during their lifetime, so that appendectomy is the most often performed abdominal surgery. For over a century, the golden standard in the treatment of patients with complicated pediatric appendicitis (CPA) was an open appendectomy. However, today many polemics among researchers are guided by whether, in pediatric
}

population, the laparoscopic approach is superior to an open appendectomy.

Objective: Comparison of the outcome of treatment of CPA between a group of patients treated with laparoscopic appendectomy and a group of patients treated with open appendectomy.

Methodology: A retrospective, clinical, comparative study was carried out covering 293 pediatric patients with an appendectomy in the period from November 2015 to April 2018 at the Clinical Center of the University of Sarajevo. Patients were divided according to the treatment method in a group of patients with open appendectomy and a group of patients treated by laparoscopic and compared with demographic and clinical data and outcomes of treatment. Results: The average time of anesthesia was 10 minutes longer in the group of patients who received laparoscopic appendectomy compared to the open appendectomy $(p=0.0086)$. The average length of hospitalization was 2 days shorter in the laparoscopic group of patients ( $p<0.0001$ ). Laparoscopically operated patients had a shorter application of parenteral analgesia compared to the patients with the open appendectomy $(p=0.0061)$. In patients with laparoscopic appendectomy the period of drainage was shorter ( $\mathrm{p}$ $<0.0001)$. No statistically significant difference was found by comparing the incidence of minor and major complications between the groups.

Conclusion: Laparoscopic appendectomy proved to be an effective method and is increasingly represented in the treatment of CPA. Children undergoing laparoscopic treatment are significantly shorter in the hospital, have a lower level of postoperative pain, a shorter period of drainage, however, longer application of anesthesia compared to children who have been operated with an open approach.

Keywords: Appendectomy, complicated, pediatric, laparoscopic, treatment outcome. 
189. Traumatic Abdominal

Trauma, Solid Organ Injury, Conservative vs Surgical Treatment

Baton KELMENDI ${ }^{*}$, Nexhmi HYSENI ${ }^{1}$, Dafina MATOSHI $^{1}$, Hysni JASHARI ${ }^{1}$, Gani ÇEKU ${ }^{1}$, Alban RUSHITI ${ }^{1}$

${ }^{1}$ Department of Pediatric Surgery, University Clinical Center of Kosovo, Pristine, KOSOVO.

\begin{abstract}
Introduction: Abdominal trauma has always been one of the major challenges for pediatric surgeon. Historically the presence of free lymph in the intraperitoneal area has been an indication of aggressive surgical treatment.

The first publication on conservative treatment for patients thought to have a limb injury was made by Simpson in 1968 . Initially, the initial treatment was initiated by Warnsborough, and this treatment was in 12 patients treated during 1956-1965, it should be noted that this has occurred in the period when ECHO or CT diagnosis was missing.
\end{abstract}

Material and methods: In the period 2017 to 2018 in our clinic we treated 14 patients with abdominal injuries. The study included lysis and heparitis injuries. Of the 14 injured 4 were subjected to surgery because of the injury, while 10 were treated conservatively.

Conclusion: Based on the series of cases that have been treated in our Clinic, we conclude that conservative treatment is the selected method of hemodynamically stable patients. The imperative is dynamic tracking and 24 hour service.

\section{Assesment on Blunt Abdominal Trauma in Children}

Agron DOGJANI MD, MSc, PhD, FACS1*, Rifat LATIFI MD, FACS, FICS ${ }^{2}$, Kastriot HAXHIREXHA MD, $\mathrm{PhD}^{3}$, Hysni BENDO ${ }^{4}$. ${ }^{1}$ Associate Professor of Surgery, University Hospital of Trauma, Lecturer at University Medical of Tirana, ALBANIA

${ }^{2}$ Professor of Surgery, Director, Department of Surgery, Program Director, Surgical Critical Care
Fellowship Chief of General Surgery, Westchester Medical Center, NY. USA

${ }^{3}$ Lecturer at State University of Tetovo, General Surgeon at Clinical Hospital of Tetovo, MACEDONIA.

${ }^{4}$ University Medical of Tirana, ALBANIA

\section{Abstract}

Introduction: Trauma is the leading cause of morbidity and mortality in the pediatrics age.
Abdominal trauma ranks third for
frequency of trauma injuries in
children.
The purpose of our study is recognition and description of the mechanisms that cause Blunt Abdominal Trauma in pediatrics age, identify the signs and symptoms that accompany it.

Material and methods: The study has retrospectively registered and includes all patients with Blunt Abdominal Trauma in pediatrics age who presented to emergency department at the University Hospital of Trauma in Tirana, Albania by 01/12/2017 $24 / 06 / 2018$. The sample in the study was taken randomly without any restriction study. The Injury Severity Score, Revised Trauma Score ... were used as the important points for evaluate gravity of injuries, and mode of treatment...

The data is introduced in absolute and percentage values, Kendal's tau b correlation coefficient and the regression analysis is used to analyze and to see the connections between the dependent versus independent variable... The type of study is control cases, with two components; descriptive and analytical...

Results: In period under study are presented about 25200 of these patients were hospitalized $6.68 \%$ cases, and in the most of the causes was motor vehicle accidents (35.4\%) and the Abdominal Trauma engaged in $25.8 \%$ of cases, in the pediatric age was $13.7 \%$. We are found connection between the injury severity score and complications rate $(\mathrm{r}=0.254, \mathrm{n}$ $=49, \mathrm{p}<0.001$ ), and injury severity score with length of stay in hospital $(r=0279, n$ $=49, \mathrm{p}<0.001) \ldots$ 
Conclusions: Blunt Abdominal Trauma in pediatrics age is a serious threat to the health of the children. Their treatment should be carried out not only tertiary trauma center but in every regional hospital...The trauma score is very valuable in the determination the gravity of the injury, mode of treatment and as a predictive tool in trauma outcomes.

Keyword: Blunt Abdominal Trauma pediatrics, abdominal injuries, trauma score, trauma assessment...

\section{Preventable and Accidental Trauma in Children}

Hysni JASHARI $^{1 *}$, Nexhmi HYSENI ${ }^{1}$, Salih GRAJCEVCI $^{1}$, Baton KELMENDI ${ }^{1}$, Murat BERISHA $^{1}$, Gani ÇEKU ${ }^{1}$

Department of Pediatric Surgery, Hospital and Clinical University Service of Pristina, KOSOVO

\begin{abstract}
Introduction: traumatic event happened is a dangerous, violent and intimidating which poses a threat to the life of the child or the child's physical integrity.

Experiencing a traumatic event that threatens the child's life or physical safety is traumatic for family members too. Despite the precautionary measures for child safety, dangerous events occur in continuity. This risk can occur outside the family, such as natural disasters, traffic accidents, violence in communities or trauma that comes as a result of domestic violence, sexual or physical violence and sudden death.
\end{abstract}

Material and Methods: During our period 2015-2018, our patients are treated with traumatic injuries of different abdominal and urological conditions, excluding those of the locomotor system and neurosurgery injuries, which are treated in the Orthopedics Clinic, respectively Neurosurgery Clinic.

Conclusion: Traumatic experiences put in motion a cascade of changes in the lives of children, experiences that can be challenging and difficult. Systematic access to injured children and adequate medical care saves the child's life.

Prevention of injuries and adequate measures to protect children are essential to reduce the number of injured children. Preventive injuries have a great financial, emotional, and social impact on sore children and their families as well as in society as a whole.

\section{The Role of Imaging in the Pediatric Spleen Trauma}

\author{
Marjeta TANKA ${ }^{I^{*}}$, Sokol BUBA ${ }^{1}$, Viola \\ SHUTERIQI ${ }^{1}$ \\ ${ }^{1}$ University Hospital Center "Mother Teresa", \\ Tirana, ALBANIA
}

\section{Abstract}

The spleen is the most frequently injured internal organ after blunt or penetrating trauma in the abdomen. The clinical evaluation of the children with blunt abdominal injury presents a really difficult and challenging task, therefore imaging plays an essential role in the diagnosing and follow up of these patients.

Ultrasonography plays an important diagnosing role especially on the hemodynamically unstable patients because it can be performed at the bedside, it is fast and noninvasive procedure. It can also help us stage the lesion, determine whether laceration or hematoma is present, determine the presence of free fluid in the abdomen, provide information of possible simultaneous damage of other abdominal organs, also Doppler US and contrastenhanced Us are useful to evaluate splenic vascularisation. However US has its limitations especially in providing information of the injury in the pelvic or lumbar spine. Therefore the gold standard in the diagnose and evaluation of hemodynamically stable patients with abdominal trauma is contrast-inhanced CT. It identifies and quantifies the injury of the visceras, the presence of intra-peritoneal and extraperitoneal fluid and blood caused by active hemorrhage, shows possible bone injury to the ribs and spine. Nowadays the 
most optimal treatment of the children with splenic injury is non operative, due to the ability of this organ to stop bleeding spontaneously. This phenomenon happens because of some unique pediatric characteristics: children have smaller blood vessels with enhanced vasocontrictive response, thicker splenic capsule, and higher proportion of myoepithelial cells. Therefore non operative treatment and regular ultrasound follow up is the first line treatment of the hemodinamically stable pediatric patients after splenic trauma.

\section{Intercourse between Trauma and Acute Scrotum (Torsion of the Test, Twist of the Epididymal Appendix)}

Saimir HETA ${ }^{1}$

${ }^{I}$ Department of Pediatric Surgery, University Hospital Center "Mother Teresa", Tirana, ALBANIA

\begin{abstract}
Acute scrotum pain, accompanied by swelling, are two clinical elements indicating the clinical presence of torsion of the test. The onset of the final diagnosis is performed after receiving the clinical history, clinical examination, analysis (complete urine, imaging), and differential diagnosis; which in this case occurs between the conditions: torsion of testicular appendicitis, epididymitis, trauma, hernias, hydrocele, varicocele of the scarring of Schönlein-Henoch. These clinical conditions do not require any further surgical intervention, as opposed to test torsion. In the differential diagnosis, the pediatric age, the pain and the explanation of episodes and the gravity of the pain, referred by the patient, and reconfirmed by the parents, help greatly. Pain as a subjective element can even be minimized by the child. The above mentioned clinical conditions can be classified in the age group when they appear: prenatal, pre-puberty or post-
\end{abstract}

pediatric age. This element helps to diagnose as soon as possible the emerging clinic. As a surgical emergency intervention, torsion of the test depends greatly on the duration of torsion, as success in testicular life depends on the sooner surgical intervention. From the daily clinic, it is noticed that in every case presented - pediatric patient - diagnosed with torsion test, always in the clinical history of the patient there is a relationship that the patient refers to with the presence of an earlier episode of testicular trauma, despite the fact that the literature is not that it supports too much logically. For clinical significance, it is worth mentioning the timely approach that clinicians need to diagnose and treat acute scrotum.

Keywords: Acute scrotum, pain, trauma, clinical diagnosis

\section{Pediatric Trauma, Triage of Pediatric Patients in Albania}

\author{
Marinela KUMARAKU ${ }^{1}$

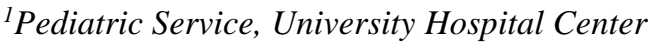 \\ "Mother Teresa" Tirana, ALBANIA
}

\begin{abstract}
Background: Injury is the leading cause of death among children older than 1 year. Each year, approximately 20,000 children and teenagers die as a result of injury. An estimated 50,000 children acquire permanent disabilities each year, most of which are the result of closed head injuries.Children present a unique challenge in trauma care because they are so different from adults - anatomically, developmentally, physiologically and emotionally.
\end{abstract}

Matherial and Methods: A study concluded that the risk of death for injured children is lower when care is provided in pediatric trauma centers rather than in nonpediatric trauma centers. Emergency medical technicians must be trained in rapid pediatric cardiorespiratory assessment, prompt establishment of effective ventilation (airway), oxygenation (breathing) and perfusion (circulation) as 
well as in stabilization and transport of injured or ill children to a tertiary care facility. EMTs are the first medical contact (first responder) that children have following an injury.

Conclusions: Triage is the process of rapid assessment of a patient with a view to define urgency of care $\&$ priorities in treatment. Triage is the first step in the management of a sick child admitted to a hospital. Caring for pediatric patients with trauma is a complex and integrated process that requires knowledge of the special considerations of pediatric trauma patients and understanding of the pathophysiology and special requirements of the pediatric population. Approach the treatment of the injured child with a rational and meticulous plan of action that not only leads to expeditious diagnosis and therapeutic intervention but also provides efficient and effective care for the patient. Keywords: Pediatric trauma, injury, children, triage, emergency.

\section{Emergency Surgery - Present and Future.}

\section{Boris E. SAKAKUSHEV ${ }^{1}$}

Medical University Plovdiv Chair of Propaedeutic of Surgical Diseases, First Clinic of General Surgery University Hospital St George Plovdiv, Plovdiv, BULGARIA

\footnotetext{
Abstract

This is an overview of Emergency Surgery concerning its content, understanding, mission, faith, suffering, challenge and satisfaction from emergency surgeons' point of view.

Traumatism is a leading global lethal factor and emergency surgery compared to planned one generates 10 to $25 \%$ more morbidity and mortality.

Terminology, definition and fundamental contribution of Emergency Surgery in terms of knowledge and practical guidelines to all key components of assessment, investigation, resuscitation and
}

surgical treatment of emergency patients are discussed.

Current tendencies of Emergency Surgery development concerning policies, social impact, resources, management, research and education are presented.

Future strategies for optimization of ongoing evolution in Emergency Surgery and patient care are envisaged.

Keywords; Emergency Surgery, morbidity and mortality, Trauma injuries...

\section{Higher Number of Colorectal Cancer Patients in our Emergency Surgical Units. Why?}

Arvin DIBRA ${ }^{1}$

${ }^{I}$ Department of Surgery, Faculty of Medicine, University of Medicine, Tirana, ALBANIA

\section{Abstract}

Colorectal cancer is the third most common type of cancer among men and the second among women. The disease shows a good prognosis when diagnosed in the early stages, where mortality is described as overall $8.5 \%$. But mortality and morbidity are lower in elective operated patients, but there is a significant increase in patients operated in emergency, as well as a reduction in 5-year survival rates.

The most common clinical presentation to the emergency units is obstruction, followed by bowel perforation. Different international studies demonstrated that around 10 to $19 \%$ of patients affected by colorectal cancer will present obstruction during the natural course of the disease. The emergency treatment is also a risk factor for a worse prognosis, with higher mortality in the short postoperative period. The difference between the two different ways of treatment consists in 15 to $30 \%$ in emergency compared to elective patients where from $1 \%$ to $5 \%$ is found. This not only because the patients' deterioration of the clinical status due to the obstructive emergency condition, but also by the advanced stage of the tumor 
found in some cases. Perforation can be the reason to emergency treatment in $3 \%$ to $8 \%$ and presents greater postoperative morbidity and mortality than colonic obstruction but seems that survival rates are similar in both situations.

This article aims to discuss our institution experience and tries to find reasons for the increasing number of colon cancer patients surgically treated in emergency regime.

Keywords: colorectal cancer, emergency surgery

\section{Emergency for Complicated Gastrointestinal Cancers}

Boris E. SAKAKUSHEV ${ }^{1}$

${ }^{I}$ Medical University Plovdiv Chair of Propaedeutic of Surgical Diseases, First Clinic of General Surgery University Hospital St George Plovdiv, Plovdiv, BULGARIA

\section{Abstract}

Gastrointestinal cancer complications include perforation, bleeding or obstruction requiring emergency surgery.

Adenocarcinoma of the stomach rarely is presented in emergency setting, because of early forthcoming local and systemic signs. GastrointestinalStromal Tumors are 47$60 \%$ of all Gastrointestinal tumors is mainly presented by upper GI bleeding ashematemesisormelena $/ 40-65 \%$ ofpatients/duetoanulceractionofgastricmuc osaoverlyingthetumor. In patientswithadvanceddisease, ontyrosinekinaseinhibitortherapystomach GIST maydeveloptumorrelatedintraluminalorintr aperitoneal, hemorrhage, rupture,

fistulaorobstructionrequiringemergentsurge ry. Completeresection (R0) isperformed, avoidingtumorruptureor wedgeresectionisadequate, iftechnicallypossible.Largetumorsnecessita tesubtotalortotalgastrectomy.

Radicalgastrectomyisnotrequired, onlycompletetumorresection, withoutlymhadenectomyand $1-2 \mathrm{~cm}$ freeoftumormargin is needed.
Almost $1 / 3$ of all CRC cases are complicated, requiring emergency treatmentforbowel obstruction, perforation or bleeding, resulting in considerable morbidity and mortality. We are often forced to perform two or even three stage operations. The emergency complicated left colon cancer often required the Hartmann's procedure, still considered as the gold standard in patients with severe performance status. Recently laparoscopic techniques, as safe and effective alternative to the conventional Hartmann's procedure are more preferred, mainly due to the better outcomes.

Keywords: Gastrointestinal Stromal Tumors, Neuroendocrine, Colorectal Cancer...

\section{Acute Pancreatitis: New Guidelines of the Management}

Enver FEKAJ ${ }^{1 *}$, Rifat BAJRAMI ${ }^{1}$, Vesel SKENDERI ${ }^{1}$, Skender ZATRIQI ${ }^{1}$, Afrim TAHIRI ${ }^{1}$, Bedri BRAHA $^{1}$, Preveza ABRASHI ${ }^{1}$

${ }^{I}$ Department of Abdominal Surgery, University Clinical Centre of Kosovo, Pristine, KOSOVO

\section{Abstract}

Recently, the original Atlanta classification of 1992 was revised and updated by the Working Group using a web-based consultative process involving international pancreatic societies. This new classification addresses the diagnosis, types of acute pancreatitis (AP), severity, and definition of pancreatic and peri pancreatic collections.

The diagnosis of acute pancreatitis involves a combination of symptoms, physical examination, and focused laboratory values. There are two different types: Interstitial edematous pancreatitis, and necrotizing pancreatitis. The diagnosis of infection is based on: ongoing signs of sepsis, CT findings of extraluminal gas within the areas of necrosis in the pancreatic and/or peripancreatic tissue, and percutaneous fine-needle aspiration. This 
new classification defines 3 degree of severity: Mild acute pancreatitis, moderately severe, and severe acute pancreatitis. There are 2 phases of acute pancreatitis: early phase, and latephase. The local complications are classified as: acute peripancreatic fluid collections, pseudo cysts, acute necrotic collections, and walled-off necrosis.

In patients with mild biliary AP, a cholecystectomy should be performed before discharge to prevent a recurrence of AP. In those with necrotizing biliary AP, a cholecystectomy is to be deferred until active inflammation subsides and fluid collections resolve or stabilize. In stable patients with infected necrosis, surgical, radiologic, and/or endoscopic drainage should be delayed preferably for more than 4 weeks to allow liquefactions of the contents and the development of a fibrous wall around the necrosis (walled-off necrosis). In symptomatic patients with infected necrosis, minimally invasive methods of necrosectomy are preferred to open necrosectomy.

Key words: acute pancreatitis, pancreatic necrosis, pancreatitis treatment

\section{Primary Anastomosis Versus Hartmann's Operation in Emergency Surgery for Acute Mechanical Intestinal Obstruction due to Left-Sided Colorectal Cancer}

Ozgur EKINCI ${ }^{1}$, Aman GAPBAROV ${ }^{1}$, Cem Ilgin EROL $^{1}$, Damla BEYAZADAM ${ }^{1}$, Tunc EREN ${ }^{1}$, Orhan ALIMOGLU ${ }^{1}$

${ }^{1}$ Istanbul Medeniyet University School of Medicine, Goztepe Training and Research Hospital,

Department of General Surgery Istanbul, TURKEY.

\begin{abstract}
Introduction: In emergency surgical cases of acute mechanical intestinal obstruction (AMIO) due to left-sided colorectal cancer (CRC), Hartmann's operation or primary anastomosis are alternative surgical methods. In this study, our aim was to
\end{abstract}

compare the clinicopathologic results and complication rates between Hartmann's operation and primary anastomosis.

Methods: Emergency surgical operations which were performed for AMIO between January 2011 and January 2018 were retrospectively analyzed. Following the exclusion of obstructive pathologies due to small intestinal cancer, right colon cancer and adhesions, left-sided CRC cases were included in the study. Demographics, operative indications, postoperative complications, and the time period between the primary operation to colostomy closure were evaluated, compared and statistically analyzed. A $p$ value less than 0.05 was accepted as statistically significant.

Results: Among the 68 patients included in the study, 48 (70\%) were men, and 20 (30\%) were women. Hartmann's operation was performed to $50(73.5 \%)$ patients (Group H), whereas primary anastomosis was carried out in $18(26.5 \%)$ patients (Group P). Group H consisted of 37 (74\%) men, and 13 (26\%) women with a mean age of 63.8 (range35-89), whereas Group P consisted of $9(50 \%)$ men, $9(50 \%)$ women with a mean age of 64.67 (range: 43-82). The most common complications in the first 6 months in Group $\mathrm{H}$ were surgical site infections (SSIs) $(\mathrm{n}=12.24 \%)$, followed by small intestinal fistulas $(n=3.6 \%)$, evisceration $(n=3.6 \%)$, colostomy revision $(\mathrm{n}=2,4 \%)$, and eventration $(\mathrm{n}=1.2 \%)$. In Group $\mathrm{P}$, an end colostomy was created in $3(15.7 \%)$ patients due to anastomotic leakage, 2 $(10.5 \%)$ patients developed SSI, and 1 $(5.2 \%)$ patient underwent surgical drainage due to intraabdominal abscess formation. There were no statistically significant difference between the two groups regarding complication rates in the first 6 months. Mean hospital stay was 10.22 (range: 1-65) days in Group H, whereas this duration was 12.94 (range: 3-61) days in Group P revealing no significant difference. Mortality occured in $6(31.5 \%)$ patients of Group P. Among the 4 (66\%) mortalities which occured in the first 
month, 2 (33\%) were due to anastomotic leakage and the other $2(33 \%)$ were due to non-surgical reasons. The remaining 2 (33\%) mortalities took place in the first year grading $(\mathrm{pN})$. There were no significant differences with regard to $\mathrm{T}$ stages. When tumor localizations were analyzed, tumors were significantly more commonly located in the descending colon in Group P, and in the sigmoid colon in Group H.

Conslusions: There were no statistically significant differences between the use of either Hartmann's operation, or primary anastomosis with regard to SSIs, other postoperative complications, or mortality rates. Therefore, given the advantage of avoiding the need for a second operation, primary anastomosis may be preferred to Hartmann's operation in cases of AMIO due to left-sided CRC.

Keywords: Hartmann's operation, colorectal cancer, colostomy revision, primary anastomosis

\section{Evaluation of the BISAP Score in Assessing Mortality and Intermediate Markers of Severity in Acute Pancreatitis}

Alma LLUKACAJ ${ }^{1}$, Etmond ÇELIKU ${ }^{1}$, Xheladin DRAÇINI ${ }^{1}$, Majlinda NAÇO ${ }^{1}$

${ }^{1}$ Department of Surgery, Faculty of Medicine, University of Medicine, Tirana, ALBANIA

\footnotetext{
Abstract

This study aims to prospectively evaluate the ability of the bedside index for severity in acute pancreatitis (BISAP) score to predict mortality as well as intermediate markers of severity in our hospital. Materials and Methods: The BISAP score was evaluated among 112 consecutive cases of acute pancreatitis admitted to our clinic between January 2011 and December 2014. BISAP scores were calculated on all cases using data within 24 $\mathrm{h}$ of presentation. The ability of the BISAP score to predict mortality was evaluated using trend and discrimination analysis.
}

The optimal cutoff score for mortality from the receiver operating curve was used to evaluate the development of organ failure, persistent organ failure, and pancreatic necrosis.

Results: Among 112 cases, there were 22 $(19.6 \%)$ deaths. There was a statistically significant trend for increasing mortality ( $\mathrm{p}<0.0001)$ with increasing BISAP score. The area under the receiver operating curve for mortality by BISAP score in the prospective cohort was $0.82(95 \%$ confidence interval: $0.70,0.95)$, which was similar to that of the previously published validation cohort. A BISAP score $\geq 3$ was associated with an increased risk of developing organ failure (odds ratio $=7.4$, $95 \%$ confidence interval: $2.8,19.5)$, persistent organ failure (odds ratio $=12.7$, $95 \%$ confidence interval: $4.7,33.9)$, and pancreatic necrosis (odds ratio $=3.8,95 \%$ confidence interval: $1.8,8.5$ ).

Conclusions: The BISAP score represents a simple way to identify patients at risk of increased mortality and the development of intermediate markers of severity within 24 $\mathrm{h}$ of presentation. This risk stratifi cation capability can be utilized to improve clinical care and facilitate enrollment in clinical trials.

Keywords: acute pancreatitis, BISAP score, mortality, evaluation

\section{BURNS: A Very Complicated "Simple" Trauma}

John E. FRANCIS ${ }^{1}$

${ }^{1}$ Lafayette Surgical Clinic, Chief of Surgery, Director of Trauma, Lafayette

\begin{abstract}
Thermal injuries of all types - hot, cold, electrical, radiation - irrevocably destroy tissue to some degree. Timing and treatment of these injuries is critical to the life-long complications related to this trauma. We will discuss current therapies, the pros and cons of treatment and timing of treatment, as well as ongoing therapies to reverse the effect of this trauma on burn survivors.
\end{abstract}


Thermal injuries cover a wide-range of presentations - from the very simple to extremely complex. Having a good understanding of the pathophysiology of burns if crucial to making good decisions in the initial treatment of burns, and for guiding long-term therapy to decrease the effect of complications associated with burns. We will discuss the current basic understanding of burn physiology and how this relates to current treatment recommendations.

\section{Advantage of Early Tangential Necrectomy in Extensive Pediatric Burns}

Kenan KARAVDIĆ ${ }^{1}$

${ }^{\text {I}}$ Clinic for Pediatric Surgery, Clinic Center of University, Sarajevo BOSNIA AND HERZEGOVINA

\begin{abstract}
Introduction: The most common causes of pediatric burns are fire, hot water, flammable liquids, gases. More than 2 million inhabitans every year in United States need help because burns. The burn wound is the source of all ill effects, local and systemic. Surgical treatment of extensive burns involves removal of necrosis and its transformation into the uncovered, uncontaminated surgical wound and covering the region with some of the grafts. This eliminates necrotic lactate lactose, improves collagen synthesis, reduces the activity of inflammatory mediators and their contribution to microcirculation and blood vessel permeability, reduces protein loss, and reduces the risk of dysthymia.

Aims of study: Determine the optimum time of operative intervention of extensively acquired pediatric patients. Determine efficacy of early tangential necrectomy in the treatment of extensive burns in children by comparing clinical parameters of patients treated with this method and patients treated with delayed tangential necrectomy or conservative (control group) method.
\end{abstract}

Material and methods: We treated 60 patients in period of 10 years. Examined group: 30 children of both sexes with extensive burns treated with surgical, early tangential necrotomy method, ages 6 to 14 years. Control group: 30 children of both half ages 6 months to 14 years, with extensive burns treated with a conservative or surgical method of delayed tangential necrectomy. In both groups we followed the next clinical parameters: body temperature, body weight, diuresis, number of days in hospital, laboratory parameters( biochemical and microbiological).

Results: The results showed that the mean values of the control group temperature (X + SEM) were significantly greater than the mean of the examined group ( $p<0.001)$, significantly lower values of serum proteins in the control group compared to the examined group ( $\mathrm{p}<0.001)$. The result showed significantly lower values of hematocrit in the control group compare to the examined group $(p=0.002)$, serum hemoglobin mean values $(X+S E M)$ were significantly lower in control compared to the study group $(\mathrm{p}<0.001)$. The results show that the median values $(X+S E M)$ of hospitalization length are significantly higher in the control group compared to the examined group $(p=0.003)$. The results showed a significantly higher incidence of burn wound infection in control compared to the examined group $(\mathrm{p}=0.007)$ and significant association between the duration of hospitalization and the number of isolated bacteria in the burns ( $\mathrm{p}=$ 0.002).

\section{Conclusions:}

-The method of early tangential necrectomy of extensively pediatric burn significantly have en effect on morbidity and mortality (improves the general condition of a patient, improves clinical and laboratory parameters, reduces the chance of early infection, reduces the possibility of causing a cause, reduces the length of hospitalization).

-The most optimal time to perform the early tangential necrotomy is the period 
between the 4th and 6th days after the injury.

-For extensively burned children over $30 \%$ of the total body surface, early tangential necrectomy should be applied on the surface of about $20 \%$ of the total body surface area as prevention of blood loss due to necrectomy and autotransplantation. -For extensively fed children over $40 \%$ of the total body surface, taking homotransplantation from parents or close relatives reduces morbidity and mortality.

Key words: pediatric, burn, early tangential necrectomy

\section{Reverse Sural Island Flap for Reconstruction of Defects of the Lower Third of the Leg and Foot}

\section{Sokol ISARAJ ${ }^{1}$}

${ }^{1}$ Service of Burns and Plastic Surgery, University Hospital Center "Mother Theresa" of Tirana, Albania

\begin{abstract}
Background: Defects of the lower third of the leg and foot pose a challenge to the surgical team. Introduction of microsurgery changed the approach to defects formerly considered even inoperable, but under conditions when expertise is missing and the possibility of free flaps is not an option, surgeons have to rely on regional solutions for each case. Aim: This article aims to evaluate the efficacy of the reverse sural island flap for reconstruction of defects of the lower third of the leg and foot and to describe our experience.

Materials and Methods: In a seven years period of time (2011-2018) 15 cases were treated with a reverse sural island flap for defects located on the lower third of the leg and foot. Flap dimension varied from $7 \mathrm{X} 4 \mathrm{~cm}$ to $15 \mathrm{X} 12 \mathrm{~cm}$, length of the pedicle from 6 to $16 \mathrm{~cm}$, two of the flaps were very close to the distal popliteal crease. In all of the cases donor area of the flap needed to be skin grafted.
\end{abstract}

Results: All the cases, except one were acute trauma cases. The remaining one was a chronic wound after a burn. In one of the cases the flap was delayed. In three of them flap was used to cover defects of the dorsum of the foot.

Conclusion: Reverse sural island flap is, as a conclusion, a very useful tool for the reconstruction of small to moderate defects of the lower leg and foot, easy to be raised, with no special needs for complicated vascular preoperative workup, and with a very low rate of failure and complications in carefully selected patients. We have found the reverse sural flap a consistent and reliable solution for most of cases with a few complications and ease of execution. Keywords: reverse sural island flap, reconstruction, defects, efficacy

\section{Epidemiology of Burned Hand in Albania 2012-2016}

Gentian ZIKAJ ${ }^{1 *}$, Gjergji BELBA ${ }^{1}$, Gezim XHEPA $^{1}$, Nardi KOLA ${ }^{1}$, Sokol ISARAJ ${ }^{1}$

${ }^{1}$ Burns and Plastic Surgery Service, University Hospital Center "Mother Teresa" Tirana, Albania

\section{Abstract}

Introduction: Burns are a major cause of injury worldwide with a high incidence on the developing country.

Aim: To give an overview of epidemiologic features and the frequency of unwanted outcome in post burning phase.

Material and methods: In this prospective study were included all patients who had combustion of the hands solely or hands accompanied with burns to other areas of the body, treated and followed up at the Burns and Plastic Service" clinic of the University Hospital Center "Mother Teresa" during the years 2011-2016. Results: Of the 333 included burn patients, $64 \%$ were males. The median age of women is 21.8 years, of men is 27.5 years and the median age total patients' population is 25.9 years. About half of patients belong to 
the age group 20-60 years (49.5\%) and only $10.2 \%$ belong to the age above 60 years. In most of the cases $(73.6 \%)$, the burn of hands is associated with burn of the other anatomical region, mostly forearm. Conclusion: The surgical treatment is used for less 30\% than of patients.

The undesirable results of the burn of hands are presented in the $33 \%$ of the patients. The contractures were the main unfavorable outcome of the burned hand.

Keywords: hand burn, epidemiology, treatment outcome

\section{Modern Approaches in the Treatment of Patients with Chronic wounds at the General Hospital DR. JOŽE POTRČ Ptuj, Slovenia}

Skender VELIU ${ }^{1}$, Jasmina KRÖPFL ${ }^{2}$, Dominika VRBNJAK ${ }^{3}$

${ }^{1}$ Surgeon, Surgical ward, General Hospital dr. JožePotrč Ptuj, Potrčevacesta 23, 2250 Ptuj, Slovenia

${ }^{2}$ Nurse for controlling hospital infection, General Hospital dr. JožePotrč Ptuj, Potrčevacesta 23, 2250 Ptuj, Slovenia

${ }^{3}$ University of Maribor Faculty of Health Sciences, Žitnaulica 15, 2000 Maribor, Slovenia

\begin{abstract}
Introduction: Chronic wounds represent an enormous health, social and economic burden in modern society. With the increasing incidence of diabetes and obesity as well as the ageing of the population, we correspondingly expect a rise in the incidence of chronic wounds. The latter will reflect in an even heavier burden for individuals, their families and the society. At the GH dr. JožePotrč Ptuj we devote a lot of time todealing with this issue, because we are aware of the impact that chronic wounds have on the quality of life of affected patients.

We have been taking care of patients with chronic wounds for a number of years in the ambulatory and hospital setting. In thisarticle we present our organizational model in the treatment of patients with
\end{abstract}

chronic wounds at General Hospital dr. JožePotrč Ptuj.

We present the modern approaches in the treatment of chronic wounds at our hospital and our results in the treatment of chronic wounds supported with clinical cases.

The key to successful management of patients with chronic wounds areprofessional competence, team work and an individual and holisticapproach to each patient.

Keywords: Chronic wounds, clinic for the treatment of chronic wounds, modern approaches in the treatment of patients, individualand holistic approach to each patient

\section{Reconstruction of Post Trauma Defects in Upper Limbs Using Perforator and Pedicled Flaps.}

Nardi KOLA $^{1 *}$, Albana ALEKSI ${ }^{1}$, Sokol ISARAJ ${ }^{1}$ ${ }^{1}$ Service of Burns and Plastic Surgery, University Hospital Center "Mother Theresa" of Tirana, Albania

\section{Abstract \\ Introductuion; During our everyday practice we face a lot of post trauma or post resection defects and open wounds with bone exposure etc, in upper limbs that are not possible treated with skin grafts or random flaps. Pedicle and perforator flaps are seen in last year's in our service as a good choice in some specific cases in order to restore length, sensitivity and function of the limb.}

\section{To be successful in such complicated procedures we should be aware of;} 1-Careful planning of the most effective flap in the area involved

2-Careful selection of the patient [age, diseases, drug and alcohol abuse] 3 -When is possible we can identify the perforator vessel or the artery of the supposed pedicle using a hand Doppler 4-In some cases a CT-Angiogram is important 
5-The consent of the patient is extremely important

Classification

Flaps are classified according to:

1-Blood supply

2-Tissue to be transferred

3-Donor site location

Our Surgeries

We will present some of our most specific cases of pedicled and perforator flaps successfully applied in our theatres. Some of them are performed for the first time in our service. Every single flap is raised respecting a meticulous technique previesly consulted with most recent literature in this field of plastic surgery. We have had our failures and this is normal but understanding our mistakes we are performing much better in our days and we will do better for the future.

Keyword; open wounds, bone exposure, skin grafts, random flaps.

\section{Inferior Limbs Amputations in Diabetic Patients Following Long-Term Artherial Complications in Vlore's Regional Hospital.}

\section{Petraq MUSTAQE ${ }^{1}$}

${ }^{1}$ Surgeon-angiologist, Vlora regional Hospital, ALBANIA

\section{Abstract \\ Introduction: In this article we are discussing the epidemic spreading of the "diabetic foot" in diabetic patients because of the peripheral vascular disease at many levels of the lower limbs (from femoral level to the foot). We have recorded 92 amputations in our Hospital from 2000 - 2015. In this study we have recorded 92 cases of lower limb amputations in both genders with 62 males and 30 females, varying from 50-80 years old. 42 of these cases have gone through a femoral amputation of one limb. 12 cases have gone through crural amputation of one or both limbs in 18 cases of Lisfranc amputations and 16 cases of atypical amputation. In this study we have also}

included 4 cases of upper limbs amputations due to microartherial complications in non-diabetic patients and patients with Raynaud syndrome.In 4 of these cases after the crural amputation we have needed a reintervention that led to the amputation of the lower limb at a higher level. Also in 8 cases with Lisfranc amputation we have needed a reintervention trying to save the calcaneous bone and in two cases we went on to amputate at crural level. In most of the cases we have performed a histological exam of tissues and vases. In

Key words: amputation, diabetic gangrene, atypical amputation, Lisfranc amputation, femoral amputation.

\section{Chronic and Infected Wounds Treatment Option with Acid Acetic}

Sulejman SOKOLI ${ }^{*}$

${ }^{I}$ Orthopedic and Trauma Surgeon, Regional Hospital of Kukes, Albania

\begin{abstract}
Introduction: Chronic wounds are those wounds that are persistent and failed to proceed through an orderly and timely reparative process to produce anatomic and functional integrity over a period of 3 months, an increase in the number of patients with chronic wounds has been reported with the population increasing in weight, advancing in age and with comorbidities. In the United States, chronic wounds affect 3 to 6 million patients each year.

The current concepts concerning the treatment of chronic wounds are focusing on fundamental treatment principles for the management of venous, arterial, diabetic, and pressure ulcers.
\end{abstract}

Material and Methods; A total of 12 patients not responding to traditional therapy enrolled in the study. A specimen of wound was isolated before local application of $1 \%$ acetic acid twice daily and after completion of treatment and processed for culture study. None of the patients received any systemic antibiotics 
during the study period and received twice daily dressings.

Results; The endpoint of the treatment was wounds free of P. aeruginosa. The duration of treatment required to eliminate the Pseudomonas from the wounds was on an average 10 days.

Conclusion: Acetic acid 1\% is a simple, safe and effective topical antiseptic that can be used in the elimination of $P$. aeruginosa from chronic infected wounds. Acetic acid 1\% is therefore recommended for effective elimination of multiple antibiotic resistant strains of $\mathrm{P}$. aeruginosa

Keywords; Acid Acetic, Infected Wounds, Chronic wounds, pressure ulcers.

\section{Do Colorectal Cancer Patients Diagnosed as an Emergency Differ from Non- Emergency Patients in Their Consultation Patterns and Symptoms}

Nuhi ARSLANI ${ }^{*}$, Jasmina VESELI ARSLANI ${ }^{2}$, ${ }^{1}$ University Clinical Center Maribor, Department for Abdominal Surgery, University of Maribor, Faculty of Medicine, Malibor, SLOVENIA.

${ }^{2}$ University Clinical Center, Ljubljana, slovenia.

\section{Abstract}

Background; Many patients with cancer are diagnosed through an emergency presentation, which is associated with inferior clinical and patient-reported outcomes compared with those of patients who are diagnosed electively or through screening. Emergency diagnosis of cancer is common and aetiologically complex.

More than $15 \%$ of colorectal cancers are diagnosed following an emergency presentation

Material and Methods: clinical study of colorectal cancers diagnosed in aur hospital 2010 and 2011 using cancer registration data individually linked to primary-care data, allowing a detailed analysis of clinical information referring to the 3-year pre-diagnostic period.
Results; Emergency diagnosis occurred in $25 \%$ and $10 \%$ of the 150 colon and 80 rectal cancers. 'Background' primary-care consultations (2-3years before diagnosis) were similar for either group. In the year before diagnosis, $>75 \%$ of emergency and non-emergency presenters had consulted their doctor, but emergency presenters had less frequently relevant symptoms (colon cancer: $38 \%$ vs $57 \%$, rectal cancer: $39 \%$ vs $52 \%$ ). Alarm symptoms were recorded less frequently in emergency presenters (e.g., rectal bleeding. However, about 1/5 of emergency presenters (15 and 19\% for colon and rectal cancers) had 'alarm' symptoms the year before diagnosis.

Conclusions; Emergency presenters have similar 'background' consultation history as non-emergency presenters. Their tumours seem associated with less typical symptoms, however opportunities for earlier diagnosis might be present in a fifth of them

Keyword: colorectal surgery, colorectal cancer, Laparoscopic surgery...

\section{Evaluation of Acute \\ Pancreatitis}

Dariel THERESKA ${ }^{1 *}$, Devid BELALLA ${ }^{1}$, Nikollaq KAÇANI $^{1}$, Arben GJATA ${ }^{1}$, Enton BOLLANO ${ }^{1}$, Inida RULI ${ }^{1}$, Anisa LIÇO ${ }^{1}$

${ }^{I}$ General and Digestive Surgery Service, University Hospital Center "Mother Theresa", Tirana, ALBANIA

\section{Abstract \\ The aim of this study is to evaluate the patients with acute pancreatitis, treated in the $3^{\text {rd }}$ Service of General and Digestive Surgery, from demographical, clinical, diagnostic and treatment point of view. Material and Methods: This is a retrospective study. Medical data extracted from patient files, diagnosed with Acute Pancreatitis, were used for its realization. This study involves 94 patients treated in the $3^{\text {rd }}$ Service of General and Digestive}


Surgery University Hospital Center, from January 2008 to December 2012.

Results: This study involves 94 patients diagnosed with Acute Pancreatitis, deriving from 16 to 89 years old. $54 \%$ of these patients were males and $46 \%$ were females. The most common clinical presentations were three: abdominal pain, nausea and vomiting. Abdominal pain and abdominal sensibility were present in all patients. To determine the diagnosis clinical, laboratory and imaging findings were helpful.

Amylase is the most frequently used test, with a level higher than three times the standard in $73 \%$ of the patients. This level has diagnostic significance for this pathology. To help in determining the diagnosis were also used blood tests, biochemical examinations and also Ultrasound, X-ray, CT-scan.

Biliary form was more evident in females, whereas alcoholic form was more evident in males. This difference had a significant statistic meaning $(\mathrm{p}=0.0005)$.

According to Atlanta criteria of gravity: 91\% light form, $9 \%$ severe form of the disease, manifested with local complications and multiple organ dysfunction syndrome.

$93 \%$ of the patients were treated conservatively, whereas $7 \%$ were treated surgically.

Conclusions: Surgical intervention was indicated in Acute Necrotizing Pancreatitis, in those cases where the patient's condition did not improve, despite conservative treatment, and in case of complications as for example Pancreatic Pseudocysts, etc. Conservative treatment includes antibiotics, electrolytes, analgesics, anticoagulants and antisecretory drugs (H2-blocker/PPI).

Keywords: Acute pancreatitis, acute necrotizing pancreatitis, abdominal pain

\section{Surgery in Colorectal Cancer Emergencies}

Devid BELALLA ${ }^{1}$, Dariel THERESKA ${ }^{1}$, Arben GJATA $^{1}$, Nikollaq KAÇANI ${ }^{1}$,

${ }^{1}$ General surgeon, 3-rd Clinic, Univesity Hospital

Center" Mother Theresa" Tirana, ALBANIA

\begin{abstract}
Background: Because of deficiencies in population screening, colorectal cancer is often detected in the advanced stages, with the first presentation at the surgical emergency. In these conditions there is an increased morbidity and mortality, treatment costs, and prolonged hospitalization.
\end{abstract}

Materials and methods: This is a prospective study of 1254 patient, operated with colorectal cancer from January 2005 January 2018 in the Clinic Visceral

Surgery, at the University Hospital Mother Teresa.

Results: Of these, 859 patients were operated with colon cancer and 368 with cancer of the rectum. 364 patients or $29 \%$ was presented as an emergency as below: Occlusion, $23 \%$ or 287 patients, perforation / peritonitis patients $51(4 \%)$, perforation / abscess 26 patients $(2 \%)$. $62 \%$, or 226 patients was operated in emergency and had primary resection and anastomose. $94(26 \%)$ patients with right colon cancer underwent to resection and primary anastomose. In $2 \%$ of cases, bypass or ileostomy was perfirmed. In 183 $(51 \%)$ patients, the cancer was localized in the left colon, sigma and upper rectum, $36 \%$ of whom were treated with resection and primary anastomose, after intraoperatory preparation of colon. The rest were treated with lateral colostomy or Hartman operation. Regarding leaks from anastomoses, we had 13 patients in 789 cases who underwent to a elective surgery, and 6 patients among those who had emergency surgery. There was no statistical difference between cases with primary anastomose between the groups of emergency and elective surgery for the patients with the tumor localized in right and left colon ( $\mathrm{p}<0.05)$. For the rectum the 
data showed a better elective surgery outcome. The SSI was found higher in the cases operated as emergency. Mortality was significantly higher in patients operated in emergency 16 (4.3\%) patients vs $9(1 \%)$. The mortality associated only to surgical factors was lower.

Conclusions: Although elective surgery has a better outcome in general, we recommend the primary anastomose in patients with colon cancer who need an emergency operation, whenever his general condition allows the procedure. The decision in cases of rectal cancer depends not only in upper factors, but also experience of the team, location and prior radiotherapy.

Keywords: colorectal cancer, emergency surgery, Albania

\section{The Advantages of one Day Surgery in Preventing of Intra- Hospital Infections}

Ilber BESIMI ${ }^{1-2}$, Ferit MUHAREMI ${ }^{1}$; Fatmir SALIU $^{1-2}$; Ilaz MIFTARI ${ }^{1-2}$; Rufadije VEJSELI ${ }^{1}$; Teuta ABAZI ${ }^{1}$

${ }^{1}$ Clinical Hospital, Department of Surgery, Tetovo, MACEDONIA

${ }^{2} S U T$, Faculty of Medical Science, Tetovo, MACEDONIA

\begin{abstract}
Introduction: The advantages of one-day surgery in the prevention of nosocomial infections are an incentive for health workers in preventing their surgery. Intrahospital infections defined as disease that developed 48 hours after hospitalization. Developed in 5-10\% of hospitalized persons. Adequate and modern treatment allows reduction intra-hospital infections. The purpose of this paper lies in the importance of one-day surgery in the prevention of intra-hospital infections, reduce use of antibiotics and provision of modern treatment.
\end{abstract}

Material and methods: The study included 133 patients operated. We divided patients into two groups: group I-66 I have dealt with one day of surgery and group II 67 patients with an average hospital stay 3.7 days. Patients were treated in 2017.

Results: In group I of the one-day surgery $77.3 \%$ were male and $22.7 \%$ females, while in group II were $73.1 \%$ male and $26.9 \%$ females. nosocomial infections in the I-st group was recorded at $10.61 \%$, while in Group II to $44.75 \%$. The most frequent cause of infections was Staphylococcus aureus intra-hospital in $79 \%$ of cases.

Conclusion: The adoption of modern trends and shorter stay in hospital prevents nosocomial infections and reduces use of antibiotics.

Key words: intra-hospital - nosocomial infections, one-day surgery

\section{Groin Pain in Sportsman}

Kastriot HAXHIREXHA ${ }^{1,2^{*}}$, Agron DOGJANI ${ }^{3}$, Lutfi ZYLBEHARI ${ }^{2}$, Ferizat DIKA HAXHIREXHA $^{2}$

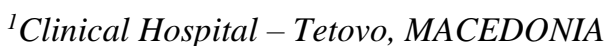

${ }^{2}$ Medical Faculty - University of Tetovo, MACEDONIA

${ }^{3}$ University Hospital of Trauma, Tirana, ALBANIA

\section{Abstract}

Introductioin: Groin pain syndrome is terms used to describe the concern commonly encountered in athletes. This syndrome consists of the pain in inguinal region usually aggravated by athletic activity and is found mostly in soccer players.

Purpose: To show our experience in the treatment of chronic groin pain in sportsman.

Material and methods: This is a retrospective review of nine football players aged between 20 and 32 years admitted in our hospital because of severe chronic pain in the region of inguinal canal.

Results: All the patients included in this study underwent surgery because of chronic embarrassing pain. In for patients we performed reinforcement of the posterior wall of the inguinal canal using 
Shouldice technique sometimes associated with neurolysis of ilioinguinal nerve and resection of the genital branch of genitofemoral nerve, whereas in five others patients the tension free hernioplasty using a prolene mesh was performed. Patients were discharged from day two to five after the operation and no any postoperative complications is observed. All the athletes returned to sports five to six weeks after operation and all of them were satisfied with the results of treatment Conclusion: groin pain in sportsmen may be a very annoying problem. In some patients the pain can be resolved with hernioplasty sometimes combined with resection of the genital branch of genitofemoral and ilioinguinal nerve (neurolysis).

Key words: Sportsman, groin pain, surgical procedure

\section{Preoperative Findings and Surgical Approach in Gastric Cancer.}

Myzafer KACI ${ }^{*}$, Drini SHEHI ${ }^{1}$, Arvin DIBRA ${ }^{1}$, Etmont CELIKU ${ }^{1}$

${ }^{1}$ General Surgery Service, University Hospital Center "Mother Theresa" Tirana, ALBANIA

\begin{abstract}
Introduction: Gastric cancer is one of the most common cancers and the second most common cause of cancer deaths worldwide. Due to its insidious clinical presentation, it is unfortunately commonly diagnosed at later stages of the disease, which is also of prognostic significance. Material and Methods: This study takes into consideration the most frequent clinical findings in correlation to different stages of Gastric cancer, consisting in data taken from General Surgery Service, University Hospital Center "Mother Theresa" Tirana during the period of time 2015-2017.The data was taken from preoperative anamnesis of 189 patients treated for Gastric cancer.
\end{abstract}

Conclusions: In this survey, the most frequent reported findings in patients first diagnosed for Gastric cancer, are weight loss and gastrointestinal hemorrhage. Considering the results of our study, the symptoms are directly linked to a late stage of the disease, which are of direct impact on surgical approach and prognosis. We conclude that it is of great importance for our healthcare system to be on alert for an earlier diagnosis for Gastric cancer patients.

\section{Terminal Ileum}

Endometriosis as a Cause of Acute Bowel Obstruction: A Case Report and Review of the Literature.

Faton T. HOXHA ${ }^{1 *}$, Shemsedin HASHANI ${ }^{1}$, Rrustem MUSA ${ }^{1}$, Shpresa HASHIMJA ${ }^{2}$, Ilir UKIMERI $^{4}$, Albulena BALOKU ${ }^{1}$, Jetlir RASHITI ${ }^{1}$, Arsim AJETI ${ }^{1}$, Ali DEVAJA ${ }^{1}$, Lulzim Q.

MALOKU $^{5}$, Ramadan SOPA ${ }^{3}$, Reshat MATI ${ }^{3}$

${ }^{1}$ Department of Abdominal Surgery, University Clinical Centre of Kosovo, Pristine, Republic of Kosovo

${ }^{2}$ Department of Anesthesiology, University Clinical Centre of Kosovo, Pristine, Republic of Kosovo ${ }^{3}$ Department of Anesthesiology, University Clinical Centre of Kosovo, Pristine, Republic of Kosovo ${ }^{4}$ Sirona Hospital, Radiology Service, Pristine, Republic of Kosovo

${ }^{5}$ Department of Gynecology and Obstetric, University Clinical Centre of Kosovo, Pristine, Republic of Kosovo

\section{Abstract \\ Background: Small Intestine affected and obstructed by endometriosis is an uncommon condition in reproductive woman years. \\ Case presentation: We present a case with acute ileum obstruction caused secondary by endometrial infiltration requiring emergency surgery as treatment of choice. Rare location of endometriosis in Ileum is diagnostic and therapeutic challenge as the signs and symptoms in acute abdominal circumstances. A pathological examination of the ileum revealed ileal endometriosis, mainly in muscularis propria and submucosal locations.}

Conclusions: We should consider the probability at reproductive female age that 
the cause of acute bowel obstruction might be intestinal endometriosis with signs of obstructions.

Keywords: endometriosis, small bowel, obstruction, intestinal resection.

\section{Recognition and Management of Extremity Vascular Trauma}

\author{
Thomas B. WHITTLE ${ }^{1}$ \\ ${ }^{1}$ Vascular Surgeon Heartland Vein \& Vascular \\ Institute, Omaha, NE, Lincoln Surgical Group \\ Lincoln, NE, USA
}

\section{Abstract}

Stabilization of the vascular trauma patient to prevent the lethal triad of acidosis, coagulopathy, and hypothermia is the initial step in the management of blunt or penetrating injury. Use of tourniquets, shunts, and blood replacement should be liberally applied. Recognition and diagnosis of specific vascular injury to the extremities requires familiarity with hard and soft physical signs of flow interruption. Mechanism of injury should be considered. There should be frequent use of ankle/brachial and brachial/brachial pressures when soft physical signs are present, and more definitive diagnostic use of MDCTA, angiography, or operative exploration when hard signs of vascular injury are present. Timing of intervention with debridement, hemorrhage control and revascularization if needed should be predicated on stability and comorbidities in the poly trauma patient. Principles for open repair include proximal and distal control, exploring the extent of injury/associated injuries, potential vein harvest for repair, and finally the repair itself. Selective use of endovascular intervention with covered stents and embolization holds future promise for vascular injuries. However, many of these patients are young, and the long-term outcomes relative to open repair has yet to be adequately evaluated.

Contraindications to endovascular repair include long segmental injuries, lack of fixation points, and arterial transection. Consideration for compartment syndrome should be made especially when there is prolonged ischemic time, associated arterial/venous injury, associated nerve/boney injury, or need for femoral vein ligation. Open fasciotomy may prevent significant renal injury or longterm limb disability in many patients.

\section{Surgical Management of Popliteal Artery Aneurysms in Albania}

Petrika GJERGO $^{1}$, Albana KENGA ${ }^{1}$, Denis KOSOVRASTI $^{1}$, Sokol XHEPA ${ }^{1}$, Edmond KAPEDANI $^{2}$, Edmond NUELLARI ${ }^{1}$.

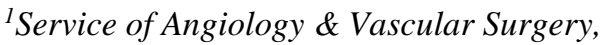
University Hospital Center "Mother Teresa", Tirana, Albania

${ }^{2}$ Faculty of Medicine, Catholic University Our Lady of Good Counsel, Tirana, Albania.

\section{Abstract \\ Background: Popliteal artery aneurysm (PAA) is uncommon, although the popliteal artery is the second most frequent location of arterial aneurysms. The aim of this study was to evaluate the epidemiology and outcomes of PAA treated surgically in our clinic.}

Methods: From 2008 to 2018, 30 patients with PAA measuring from 2 to $7 \mathrm{~cm}$ were operated in our department. All data were collected retrospectively. All patients, except one, had undergone ultrasonography examination and computed tomography angiography before treatment. Patients had a 30-day follow-up with clinical and ultrasound examination.

Results: Median age of the patients was 68 years all patients were male. PAA were asymptomatic in 13 patients (43.3\%), 1 patient had intermittent claudication, 1 patient presented with rupture of popliteal aneurysm, while the other patients $(50 \%)$ had acute ischemia due to acute thrombosis in 11 patients, chronic thrombosis in 2 patients and distal embolization in 2 patients.

From 30 patients, only one patient underwent thrombolysis with reteplase, all the others were surgically treated. 26 patients were treated with aneurysmectomy 
with prosthetic graft interposition using a posterior approach. In 2 patients, distal ligation of the aneurysm with femoralpopliteal bypass grafting was performed. Only one patient underwent trombectomy. During the follow-up period (30 days), no deaths and no graft thrombosis were registered. There was only one limb amputation (the patient who performed trombectomy) and one fasciotomy (the patient that underwent thrombolysis). The limb salvage rate was $96,7 \%$.

Conclusions: PAA are rare, only 30 patients were diagnosed and treated during the last 10 years in our clinic. The surgical treatment of PAA had excellent short-term results with no mortality and a high limb salvage rate.

Keywords: Popliteal artery aneurysm, intermittent claudication, aneurysmectomy, trombectomy.

\section{Vascular Access to Hemodialysis the Creation of Arteriovenous Fistula for Hemodialysis is the Best Choice for People with Advanced Stages of Renal Disease.}

\author{
Edmond NUELLARI ${ }^{1 *}$ \\ ${ }^{1}$ Service of Angiology \& Vascular Surgery, \\ University Hospital Center "Mother Teresa", \\ Tirana, Albania
}

\section{Abstract \\ Introduction; Primary arteriovenous (AV) fistulas have been done in patients with renal disease at the last stages of hemodialysis. The purpose of the study was to record Fistula $\mathrm{AV}$ for hemodialysis is the first and best choice in the patients in the open stages of renal disease.}

Materials and Methods: The actual study was conducted at the Vascular Surgery Service in Tirana. There was no flux of blood and persistence and function of fistula during hemodialysis.All patients were operated under local anesthesia.

Results:In our study showed that $77 \%$ of the AV fistulas performed in the radial arteries with side by side anastomoses, and $80 \%$ of those with end by side anastomosis were functionally no problem in the first year. Within two years, the lateral and lateral anastomosis rate was 50\% and 55\% respectively. In addition, the rate of function was $90 \%$ in FAV made in the brachial artery with end-to-side anastomosis, whether it was primary or secondary, at the end of the first year. However, rapid fastening was observed at the rate of function during the third year both in radial arteries and in brachial arteries based on FAV.

Conclusions: We confirmed that arterial surface arterial veins in AV primary fistula were the optimal and rational approach to hemodialysis, providing a sufficient flow of blood during this process. In addition, FAV failure, the primary fistula should be replaced by FAV, secondary fistula is preferably done with the brachial artery.

Keywords; arteriovenous fistulas, hemodialysis, renal disease, Vascular Surgery

\section{Treatment Options of Crural Pseudo Aneurysms. A Cases Series}

\author{
Edmond NUELLARI ${ }^{1 *}$, Albana KENGA ${ }^{1}$, Denis \\ KOSOVRASTI $^{1}$, Petrika GJERGO ${ }^{1}$, Edison \\ HALILI $^{1}$, Sokol XHEPA ${ }^{1}$ \\ ${ }^{1}$ Service of Angiology \& Vascular Surgery, \\ University Hospital Center "Mother Teresa", \\ Tirana, Albania
}

\begin{abstract}
Introduction; Pseudo aneurysms (PAs) of crural arteries represent rare complications after vascular interventions or surgery. Management of crural PAs includes different treatment options, conservative treatment as well as open syrgery or endovascular procedures. We reviewed our experience.
\end{abstract}

Material and Methods; We retrospectively analysed all patients who were diagnosed with crural PAs in our institution since 2000. We evaluated all PAs for etiology, treatment and outcome. Endpoints were: target vessel patency, vascular reintervention and limb loss. 


\section{Results.}

A total of 30 patients were diagnosed with crural pseudo aneurysms. Pa was caused by vascular interventional in 24 patients (80\%): in 22 of them open balloon thrombectomy had been performed, one patient had sub fascial endoscopic perforator vein surgery and one patient transcutaneous catheter with balloon assisted. In 6 patients (20\%) the PA was caused by an orthopedic surgical procedure.

Location of crural Pas: Peroneal artery ( $\mathrm{n}$ $=11: 36,7 \%)$, posterior tibial artery $(n=10$ : $33,3 \%)$, anterior tibial artery $(\mathrm{n}=5$ :

$16,7 \%)$, and tibio-peroneal trunk $(n=4$ : 13. 3\%). Treatment of crural Pas included: open surgery $\left(\mathrm{n}_{=} 13: 43.3 \%\right)$. endovascular procedures $(\mathrm{n}=6: 20 \%)$, and conservative management $(\mathrm{n}=11): 39.7 \%)$. After a median follow-up of 7 months (range: 0-36 months) 5 of 6 endografts were occluded. None of the surgically, endovascularly and conservatively treated patients needed reintervention for crural Pas.

\section{Complicated Varicose Veins Still Need Surgical Treatment}

Sokol XHEPA ${ }^{1}$, Edmond NUELLARI $^{1}$, Petrika GJERGO $^{1}$, Albana KENGA ${ }^{1}$, Denis KOSOVRASTI ${ }^{1}$, Marsela SOPIQOTI ${ }^{1}$ ${ }^{1}$ Service of Angiology \&Vascular Surgery, University Hospital Center "Mother Teresa” of Tirana, Albania

\section{Abstract \\ Background: Radiofrequency or Surgical Treatment? \\ This is the question today for the management of the varicose veins. We think that huge and complicated varicose veins should be treated surgically.}

Patients and Methods: During the period January 2007 - January 2017 we performed surgical treatment for all the patients with:
1. diameter of the great saphenous vein (GSV) more than $20 \mathrm{~mm}$,

2. thrombosis of GSV, especially at the sapheno femoral junction,

3. aneurysm of GSV before sapheno femoral junction,

4. ruptured primary varicose veins with huge collaterals.

These cases do not benefit of the advantages of the Radiofrequency: no pain, no general or spinal anesthesia, no recovery in the hospital, 35-40 minutes procedure in office, next day back to work. Results: All the patients had no hemorrhagic, thrombotic or embolic complications. According to the skin damages in the presentation, they had improvements of the quality of the skin. Conclusions: We should indicate the right procedure for every case. $70 \%$ of cases in Albania are complicated or with huge GSV (bigger than $20 \mathrm{~mm}$ ). If we indicate the wrong procedure, the bad results will come back like a boomerang.

Keywords: varicose veins, sapheno femoral junction, Radiofrequency

\section{Ruptured Abdominal Aortic Aneurysm Associated with left Colon Gangrene and Neurological Disorders. A very rare Combination}

\author{
Sokol XHEPA ${ }^{1 *}$, Gentian $\mathrm{CACO}^{1}$, Petrika \\ GJERGO $^{1}$, Myzafer KAÇI ${ }^{2}$, Stavri LLAZO ${ }^{3}$, Ervin \\ $\mathrm{BEJKO}^{3}$

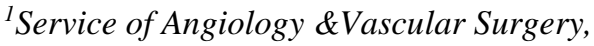 \\ University Hospital Center "Mother Teresa" of \\ Tirana, ALBANIA \\ ${ }^{2}$ Service of General Surgery, University Hospital \\ Center "Mother Teresa" of Tirana, ALBANIA \\ ${ }^{3}$ Service of Anesthesiology \& Intensive Care, \\ University Hospital Center "Mother Teresa" of \\ Tirana, ALBANIA
}

\begin{abstract}
Background: To report the management of a very rare combination of two severe surgical emergencies, ruptured abdominal
\end{abstract}


aortic aneurysm (rAAA) and gangrene of the left colon, each of them with a high mortality (rAAA $48.5 \%$ ).

Case report: A 59-year-old woman was presented at the Service of Emergencies at UHC "Mother Teresa" of Tirana on January 4, 2013, transferred from Durrës Hospital with the diagnosis of rAAA based on an unclear CT. She arrived in a profound hypovolemic shock after she had a cardiac arrest and resuscitation at the ICU of Durrës Hospital (no information about anoxic brain time).

After a brief compensation of volume in our ICU, we took the patient in the operation room. Intraoperatively we found an infrarenal rAAA and a gangrene of sigmoid and left colon.

We performed the resection of the aneurysm and the reconstruction of the aorta with the interposition of a tub graft. Immediately thereafter, the general surgeons performed a large left hemicolectomy with temporary colostomy (after 3 months they performed the second step, colo-rectal anastomose).

Postoperatively she remained 3 weeks in the ICU, mostly due to neurogical complications after the ischemic stroke she had in Durrës Hospital, and on January 29, 2013 she returned to the Service of Neurology at Durrës Hospital for the neurogical rehabilitation.

After 1 year the patient come back for consultation, she was totally healed for both surgical pathologies and she had a complete central and peripheral neurogical rehabilitation.

Conclusions: Gangrene of the left colon after rAAA is a very rare and severe combination caused by the acute occlusion of the inferior mesenteric artery in the presence of hypovolemic shock and insufficient collateral circulation. The strategy of treatment includes: fast diagnosis, short hypotensive resuscitation, cell-saving and auto-transfusion, and the simplest possible surgical reconstructions for both pathologies.
Keywords: Colon Gangrene, temporary colostomy, peripheral neurogical

\section{Risk Factors Associated with Severe Carotid Artery Stenosis in Candidates for Elective Coronary Artery Bypass Grafting Surgery}

Petrika GJERGO ${ }^{1}$, A. VESELI ${ }^{1}$, H. GJERGO ${ }^{1}$, A. SIMAKU $^{2}$, Edmond KAPEDANI ${ }^{3}$, Edmond NUELLARI ${ }^{1}$

${ }^{1}$ Service of Angiology \& Vascular Surgery, University Hospital Center "Mother Teresa", Tirana, Albania

${ }^{2}$ Public Health Institute, Tirana, Albania

${ }^{3}$ Faculty of Medicine, Catholic University Our Lady of Good Counsel, Tirana, Albania.

\begin{abstract}
Background: The identification of carotid artery stenosis in patients with coronary artery disease prior to coronary bypass surgery $(\mathrm{CABG})$ can reduce morbidity and mortality from stroke. Therefore, a preoperative carotid duplex ultrasonography, which is simple, noninvasive and inexpensive may be necessary.

The purpose of this study was to evaluate the prevalence and risk factors associated with severe carotid artery stenosis (CAS) in CABG patients.
\end{abstract}

Material and methods: 49 patients with carotid stenosis $\geq 70 \%$ identified by duplex ultrasound were matched by age and sex to control patients with carotid stenosis $<50 \%$ $(n=49)$. A total of 405 patients hospitalized for elective $\mathrm{CABG}$ were screened to arrive at these patients' cohorts. Medical records of all 98 patients were reviewed for the presence of a carotid bruit, atherosclerotic risk factors, history of a prior cerebrovascular event and extent of coronary artery disease. Patients with previous $\mathrm{CABG}$, carotid artery endarterectomy or stenting were excluded. Results: Prevalence of severe carotid artery stenosis was $12.1 \%$. The patients' cohorts were predominantly male $(85.7 \%)$ with mean age 65 years. There was no 
significant difference between groups regarding the prevalence of atherosclerotic risk factors or prior cerebrovascular events. The extent of coronary artery disease including left main coronary artery disease did not differ significantly between groups. Multivariate analysis revealed that the presence of a carotid bruit (OR 94.72, 95\%CI:16.49-543.85, $\mathrm{P}<0.0001)$ and smoking (OR 6.21, 95\% CI:1.15-33.58, $\mathrm{P}=0.034$ ) were associated with an increased risk for severe CAS.

Conclusions: This study showed a significant proportion of severe carotid artery stenosis in our patients undergoing CABG. The presence of a carotid bruit and smoking resulted independent predictors of severe carotid disease.

Keywords: carotid artery stenosis, coronary bypass surgery, carotid artery stenosis...

\section{Acute Coronary Syndrome in the Emergency Department.}

Migena ISHMAKEJ ${ }^{1}$

${ }^{1}$ University of Medicine Tirana, ALBANIA

\begin{abstract}
Background: Acute coronary syndrome (ACS) is a major cause of morbidity and mortality worldwide and common cause of attendance and admission to emergency services. It encompasses a group of entities including acute myocardial infarction with ST-segment elevation, myocardial infarction without ST-segment elevation and unstable angina.
\end{abstract}

The aim of this study is to provide epidemiological information of ACS, risk factors and pain characteristics as a presenting symptom.

Materials and Methods: This is a hospitalbased prospective study comprising all the patients who had visited the emergency department between 1 March and 30 May $2018(\mathrm{~N}=895)$ and 200 patients hospitalised in the University Medical Center of Tirana "Mother Teresa". The patients were randomly selected and data was collected based on a form within 48 hours of admission in the emergency department.

Results: Of the total, $73 \%$ of the patients diagnosed with ACS were males and 27\% females. Unstable Angina was observed in $52 \%$ of the cases, non-ST elevation myocardial infarction in $13 \%$ and STelevation myocardial infarction in $35 \%$ of the patients. The mean age of the patients was 65.1 years. Hypertension as a risk factor for coronary disease was found among $81 \%$ of patients and diabetes in $29 \%$ of the cases. $52 \%$ of the patients were smokers and $17 \%$ consumed alcohol. The majority of patients $(57 \%)$ were overweight. Dyslipidemia was found in $61 \%$ of patients while family history was positive in $22 \%$ of patients. Pain as a presenting symptom was found among $93,5 \%$ of patients with an intensity evaluated with 7 from males and 9 from females.

Conclusions: The diagnosis of acute coronary ischemia depends upon the characteristics of the chest pain, specific associated symptoms, abnormalities on electrocardiogram (ECG), and levels of serum markers of cardiac injury. A patient with a possible ACS should be treated rapidly. Thus, initial management steps must be undertaken before or during the time the diagnosis is being established.

Keywords: Emergency Department, Acute coronary syndrome, diagnosis, management

\section{Blunt Abdominal Trauma in Geriatric Patients.}

\author{
Amarildo BLLOSHMI ${ }^{1}$, Agron DOGJANI ${ }^{2}$ \\ ${ }^{1}$ University of Medicine Tirana, ALBANIA \\ ${ }^{2}$ General Surgery and Trauma Service, University \\ Hospital of Trauma, Tirana, ALBANIA.
}

\begin{abstract}
Background: Third age population (over 65 years old) is continuously increasing in our country due to increased life expectancy and improved health care system. Evidence based studies have clearly proven that geriatric population
\end{abstract}


have a worse prognosis than younger population after a blunt abdominal trauma (BAT).

The aim of this study is to make a complete evaluation of blunt abdominal trauma in geriatric population and compare it with blunt abdominal trauma in younger population.

Materials and Methods: This is a comparative and retrospective study conducted in the time frame November 2017 - January 2018, in the University Hospital of Trauma (UHT). First we divided patients in two categories, BAT and nBAT. Everybody who arrived dead to hospital or died shortly after arriving was excluded from the study.

Results: BAT patient were than divided into three categories: BAT in children (014), BAT in adults (15-65) BAT in elders (>65). Variables analysed are: age, sex, trauma mechanism, shock index (ALLGOWER formula), Injury Severity Score (ISS), Revised Trauma Score (RTS), hospitalisation time, transportation, treatment of choice, damaged organs, etc. Than each variable was compared between populations to make the changes between them visible.

Conclusions: Evaluation of BAT in elderly clearly shows that these patients have poorer outcome compared to younger patients. This is linked to physiologic changes that occur with age and chronic illnesses that are very common in this age. Keywords: Blunt Abdominal Trauma, Geriatric patients, ISS, RTS

\section{Intramedullary Nailing in the Treatment of Distal Tibia Fractures}

Teodora TODOROVA ${ }^{1}$, Ajruli B ${ }^{1}$, Rezeart DALIPI $^{1}$, Aleksandar SAVESKI ${ }^{1}$, Aleksandar TRAJANOVSKI ${ }^{1}$, Antonio GAVRILOVSKI ${ }^{1}$, Samardziski M ${ }^{1}$, Simon TRPESKI ${ }^{1}$, Ilir HASANI ${ }^{1}$, ${ }^{1}$ University Clinic for TOARILUC, Skopje, MACEDONIA
Abstract
Introduction: distal tibia fractures are common injuries in traumatology, usual by frequency and often followed with complications. These injuries are classified in extraarticular fractures, partial fractures and complete articular fractures of the distal tibia. In this study, by following a group of patients in which these types of traumas are treated with intramedullary fixation of tibia, we processed the outcome, complications, healing and function retrieval of the injured region of the extremity after the occurred trauma and after its surgical correction.

Aim: to evaluate functional results at patients after their surgical treatment of distal tibia fractures with intramedullary nail.

Material and Methods: This clinical study was performed in the University Clinic of Traumatology in a two year period of time and 27 patients were included. To appraise lower extremity function at the patients, we used Olerud Molander score system and we assigned dorsiflexion.

Results: in our study we included 27 patients with extraarticular fractures of distal tibia. Average dorsiflexion is measured 18,96 $\pm 3,7$ degrees, while Olerud Molander score for distal tibia function is $82,41 \pm 16,7$. Two complications occurred. Also an analysis of results based on AO classification was made.

Conclusion: The surgical as well as the postoperative treatment of distal tibia fractures represents a challenge, primarily because bone and wound healing can be critical. Intramedullary nailing allows minimally invasive, symmetric and dynamic fracture fixation, following the principles of biological fracture fixation, and is therefore the treatment of choice in distal tibial fractures. The obtained results in this study showed good postoperative results and low complications rate.

Key words: intramedullary nailing, distal tibia. 


\section{Considerations and Evaluations on Abdominal Trauma in Pediatric Age.}

Hysni BENDO ${ }^{2 *}$ Agron DOGJANI ${ }^{1}$,

${ }^{I}$ General Surgery and Trauma Service, University Hospital of Trauma, Tirana, ALBANIA.

${ }^{2 *}$ University of Medicine Tirana, ALBANIA

\begin{abstract}
Background: Trauma is the leading cause of morbidity and mortality in the pediatrics age.

Most of the injuries are occupied by craniocerebral trauma followed by locomotor trauma. Abdominal trauma ranks third for the frequency of trauma injuries in children.

The Aim of our study is to evaluate and review the mechanisms that cause Blunt Abdominal Trauma in pediatric age, identify the signs and symptoms that accompany it, clarify the importance of using scoring systems and to discuss operative or non-operative management criteria.
\end{abstract}

Material and methods: The study has retrospectively registered and included all patients with Blunt Abdominal Trauma in pediatric age who presented to emergency department at the University Hospital of Trauma in Tirana, Albania by 01/12/2017 $24 / 06 / 2018$. The sample in the study was taken randomly without any restriction. Other variables included are: age, gender, mechanism of injury, the examinations performed and timing of their treatment tactics. The level of ISS was used as a measure for measuring gravity of injuries in severe cases with ISS $>15$.

Results: The study included 49 pediatric patients of age 1 to 16 years old. Males consisted of $39(80 \%)$ patients, while 10 (20\%) patients were females. The main reason of trauma were motor vehicle accidents (55\%) followed by falls from height $(33 \%)$ and injury by solid objects (6\%).

Pediatric patients comprised $13.7 \%$ of all trauma cases presented at the hospital during the study period.
Conclusions: Blunt Abdominal Trauma in pediatrics age is a serious threat to the health of the children. Their treatment should be carried out not only in national trauma centre but in every regional hospital or even in smaller hospitals, rigorous enough to apply all protocols of trauma, particulary in children.

Keywords: Blunt Abdominal Trauma, pediatric, trauma and abdominal injuries, polytrauma

\section{Management of Blunt Liver Trauma}

Fatjona SHEHU ${ }^{1}$ Agron DOGJANI ${ }^{2}$

${ }^{1}$ University of Medicine Tirana, ALBANIA

${ }^{2}$ General Surgery and Trauma Service, University Hospital of Trauma, Tirana, ALBANIA.

\begin{abstract}
Background: Serious injuries to the nerve, venous and hepatic portals triad continue to remain a challenge for surgeons, as these damages are a leading cause of mortality. Medicine development in the field of imaging and surgery has evolved the thought of hepatic trauma treatment in terms of conservative treatment.

This study aims to describe the causes of trauma, the degree of injury, the chosen method of treatment, the success rate and the developed complications; comparing the results of our study with the results of literature.
\end{abstract}

Material and methods: The study is of a retrospective character and includes all patients with hepatic trauma, presented in the Emergency of Politrauma in SUT, Tirana, from December 2017 to May 2018. The study sample was taken randomly, without any study restriction.

Results: During the 6 month period, 71 patients with hepatic trauma were reported, of whom $52(73.2 \%)$ with blunt hepatic trauma and 19 (26.8\%) with penetrating injuries. Men (67.3\%) were more affected than females $(32.7 \%)$ with a male female ratio of 2: 1 . The most common cause of the injuries was car accident, while the 
most affected age group was 16-35 years old. In 52 patients with blunt hepatic trauma $53.8 \%$ were treated conservatively and $46.2 \%$ surgically.

Conclusions: Conservative treatment is the chosen method for the management of blunt hepatic trauma in hemodynamically stable patients. The conservative method significantly improves the outcome compared to surgical management, in terms of decreased abdominal infections, decreased transfusions, and decreased hospitalisation period. However, hemodynamically unstable patients with peritonitis should undergo immediate surgical intervention.

Keywords: Blunt Liver Trauma, Management, Non-Operative Management (NOM)

\section{8. $\quad$ Treatment of Lower Leg \\ Fractures.}

Ornela ÇELA ${ }^{1}$

${ }^{1}$ University of Medicine Tirana, Albania

\begin{abstract}
Background: Tibial fractures are one of the most common injuries treated by orthopaedic surgeons. There is a wide variety in the patterns of injury and treatment.
\end{abstract}

The aim of this study is to give a complete picture of treatment and epidemiology of lower leg fractures in UHT (University Hospital of Trauma), Tirana.

Materials and Methods: This is a descriptive and retrospective study conducted in the time frame January 2017 - December 2017. We analyzed 100 patients with lower leg fractures diagnosed in the Orthopaedics Service in the UHT. Recorded data include aetiology, age, gender, type of fracture, location of fracture, treatment of choice, hospitalisation time and early complications.

Results: In UHT the treatment of Tibial injuries is made surgically in $89 \%$ of cases and conservatively in $11 \%$ of cases (cast). Surgical methods of treatment are: intramedullary nails, osteosintesis with plates and screws and external fixation. The most used treatment in SUT is osteosintesis with plates and screws (62\% of cases).

Conclusions: Most orthopaedic surgeons agree that treatment has to be individualized and matched to the patient's injury pattern, medical status at the time of the injury, the cause and severity of the injury, the presence or extent of soft tissue damage that surround the tibia. In many cases, a tibial fracture will be successfully managed without complications. Recovery from a tibial fracture varies based on the severity of the fracture. A person will often recover within 4 to 6 months. Recovery time may be longer for a complete break compared to a partial one and may take longer if a person has poor health for other reasons.

Keywords: lower leg fracture, tibia, fibula, treatment

\section{General Consierations on Blunt Abdominal Trauma.}

Granit RAMIZI ${ }^{1}$, Agron DOGJANI ${ }^{2}$

${ }^{1}$ University of Medicine Tirana, Albania

${ }^{2}$ General Surgery and Trauma Service, University

Hospital of Trauma, Tirana, Albania.
Abstract
Background: Trauma or injury has been defined as damage to the body caused by an exchange with environmental energy that is beyond the body's resilience. Blunt abdominal trauma (BAT) is a leading cause of morbidity and mortality among all age groups. Most common causes of blunt abdominal trauma are automobile accidents, falls, assaults and industrial accidents. Identification of serious intra- abdominal pathology is often challenging; many injuries may not manifest during the initial assessment and treatment period.
The aim of this study is to discuss the etiology, epidemiology and pathophysiology of blunt abdominal 
trauma, as well as its clinical presentation, diagnostic and treatment modalities. We also aim to give a review of literature

Material and Methods: This is a retrospective study conducted during May 2017 - June 2018 time frame. The study included 77 patients with Blunt Abdominal Trauma admitted to the University Hospital of Trauma (UHT), Tirana, Albania.

Results: The study included 16 (20.8\%) females and 61 (79.2\%) males of all age groups. Most common mode of injury was road traffic accidents comprising 46 cases (59.7\%), followed by fall from heights comprising 19 cases $(24.7 \%)$, and hit by solid objects in 12 cases (15.6\%).

Conclusions: Morbidity and mortality can be prevented by timely initial resuscitation and correct diagnosis as well as management (operative or non-operative) which depend on patient's hemodynamic stability and findings of imaging studies. Keywords: Blunt abdominal trauma, Nonoperative management, Road traffic accident

\section{Management of Blunt Splenic Trauma}

Liridon NUREDINI ${ }^{1}$

${ }^{1}$ University of Medicine, Tirana, Albania

\begin{abstract}
Background: Spleen is the most damaged abdominal solid organ in Blunt Abdominal Trauma (BAT) and Toraco-Abdominal Trauma (TAT) with $60 \%$ of damages that result in increased morbidity and mortality. The aim of this study is to discuss epidemiological and physiopathological elements of the Splenic Trauma (ST) at the University Hospital of Trauma (UHT), its management and give a literature review.

Materials and Method: This study has a combined character (prospective and retrospective) and includes all the patients presented in the UHT emergency department in Tirana, from May 2017 to June 2018. Patients in the study were
\end{abstract}

chosen randomly without any study restriction. There The medical records of the traumatized patients at the moment of their arrival were considered, as well as the appropriate medical guides and surgical techniques, comparing them with the literature records.

Results: 91 patients are included in our study, from these $76 \%$ Males and 24\% Females. Most of them were over 15 years old $(40.44 \%)$. Automobile Accidents (AA) were the cause of $57 \%$ of cases, Fall from Highs (FH) accounted for $26 \%$ and Injury by Solid Objects (ISO) 17\%.

Conclusions. It is very important for all emergency doctors at all cases with BST ( Blunt Splenic Trauma), for its management to rely primarily on the clinical data, injury mechanism, time of hospitalization, examinations like abdominal ECHO, CT (Computed Tomography), MRI, etc. A high index of clinical suspicion along with radiological diagnosis helps to identify and characterize splenic injuries with high accuracy and is useful for timely decisionmaking to choose between OM or NOM. Careful selection of NOM is associated with high success rate with a lower rate of morbidity and mortality.

Keywords: Spleen injury, blunt trauma, management

\section{Comparison between Ravitch and Nuss Procedure as Surgical Treatment Options for Pectus Excavatum}

\author{
Doruntina KLENJA ${ }^{1}$, Anila BELCHISHTA ${ }^{1}$, Risto \\ CHOLANCHEVSKI $^{1}$ \\ ${ }^{1}$ University Clinic for Orthopedic Surgery-Skopje, \\ MACEDONIA
}

\begin{abstract}
Pectus excavatum (PE) is the most common congenital chest wall anomaly. The deformity is reported to occur more frequently in males than females. PE is sporadic and generally asymptomatic. The Haller index is a mathematical relationship that exists in a
\end{abstract}


human chest section observed with a CT scan and describes the extent/depth of the pectus: mild, moderate or severe.

The two most common types of surgeries used to correct pectus excavatum is the open repair (Ravitch) or the minimally invasive repair with a metal bar (Nuss) procedure.

Aim of this study is to compare results between Ravitch and Nuss procedure. The comparison of patients that underwent repair of a pectus excavatum during includes a 10-year period from 2005 to 2015. The cases were divided into two groups, group I operated with Ravitch procedure and group II with Nuss procedure.

In our cohort the inclusion criteria were CT chest with moderate $\mathrm{HI}>3.25$, bony deformity distressing the patient psychologically, Progressive bone abnormality, bony deformity with progressive symptoms secondary to compression either cardiac or pulmonary with positive finding in EKG,

Echocardiography or pulmonary function study.

With Ravitch procedure are operated 19 patients and with Nuss procedure 11 patients. Only $45 \%$ from Ravitch and 55\% patients operated with Nuss procedure show better results.

As conclusion wounds infection from Ravitch are more significant, as for bar displacement shows better results at Nuss procedure. 


\section{POSTER PRESENTATIONS}

\section{Procalcitonin value guided treatment of lower respiratory tract infections.}

Altin HABAZAJ ${ }^{1 *}$, Vrenoz HODAJ ${ }^{2}$

${ }^{1}$ Pneumology Sevice, University Hospital of Trauma, Tirana Albania.

${ }^{2}$ Emergency Department, University

Hospital of Trauma, Tirana Albania.

\begin{abstract}
Introduction;Acute respiratory infections (ARIs) compriseof a large and heterogeneous group of infections including bacterial, viral, and other etiologies. In recent years, procalcitonin (PCT), a blood marker for bacterial infections, has emerged as a promising tool to improve decisions about antibiotic therapy (PCT- guided antibiotictherapy). Distinct from CRP and other acute-phase reactants, existing data suggest that PCT levels rarely increase in response to viral infections, indicating that PCT may be useful for discrimination between bacterial and viral infections. Procalcitonin is a precursor to calcitonin. The final step in the synthesis of calcitonin is inhibited by cytokines and endotoxin released during bacterial infections; therefore, procalcitonin levels are selectively elevated in patients with bacterial infections.

The use of procalcitonin to guide initiation and duration of antibiotic treatment results in lower risks of mortality, lower antibiotic consumption, and lower risk for antibioticrelated side effects.

The FDA recently approved a procalcitonin assay for use in hospitals and EDs to help prescribers determine if an
\end{abstract}

antibiotic should be prescribed and for what duration.

\section{Pigmentation Disorder and Dermatology Life Quality}

Ardiana SINANI ${ }^{1}$, Dorela VASHA ${ }^{2}$, Rovena LICO $^{3}$

${ }^{1}$ Military Medical Unit at University Hospital of

Trauma, Tirana, Albania

${ }^{2}$ Health Inspectorate, Preventive Medicine at the

Ministry of Defense, Tirana, Albania.

${ }^{3}$ Speciality Policlinc No 1, Tirana, Albania

\section{Abstract \\ Introduction: Vitiligo is an acquired chronic depigmenating disease of the skin. It causes loss of pigment on effected areas of the skin or mucosae and is characterised by milk white, nonscaley with distinct margins.}

Objective: Description of vitiligo.

Different clinical forms, and the most frequent consequences of the disease in the social life of the individual.

Results of treatment of simple cases and complex cases. There is very limited information available in the textbooks on vitiligo. This is up-to-date literature overview of the disease including definition, classification, aetiology, histopathology, prognosis, assessment and treatment There is a current lack of consensus in the definition and methods of assessment of this disorder, which makes it difficult to compare the outcomes of different studies of the same treatment. Vitiligo is supposed to be an multifactorial disease in which melanocits are destroyed and this results in the absence of pigmentation in affected areas. Even though most people with vitiligo are in good general health, they face a greater risk of having other diseases such as diabetes, thyroid disease, pernicious anaemia (B12 deficiency), adrenal gland disease and alopecia areata.

Conclusion: Skin diseases are very common, affecting over a quarter of the population. Although usually not lifethreatening, skin diseases have a significant impact on the quality of life of 
patients and cause considerable psychological distress. Vitiligo, a condition which results in the loss of pigment from the skin and the cosmetic disfigurement of this seemingly inconsequential skin disease has a major impact on the quality of life of patients especially for people with darker skin. Finally, the aetiology and pathogenesis of vitiligo remains unclear. It is still not understood what causes the destruction of melanocytes. Also, uncertainties remain about the natural history and epidemiology of this disease. A variety of methods for repigmenting the skin of people with vitiligo have been tried in but vitiligo treatment is a major challenge If a cure for vitiligo is to be found, further research is required in understanding the aetiology, epidemiology and natural history of vitiligo. Current treatments help to alleviate symptoms for temporary repigmentation of vitiliginous patches, but these do not cure the underlying disease.

Keyword: Vitiligo, chronic depigmenating disease, pernicious anaemia,

\section{Cerebral Coma as a Rare but Serious Complication During Epidural Anesthesia. A Case Report.}

Dritan TODHE ${ }^{1}$

${ }^{1}$ Orthopaedic and Trauma Service, University

Hospital of Trauma, Tirana, Albania

\begin{abstract}
Background: The routine use of epidural anesthesia has become a standard procedure during the prosthetic surgery in hip and knee replacement.

Case presentation: An 80-year-old man was subject to a double hip replacement (bilateral) at a single operative procedure. Epidural anesthesia was used. The anesthesiologist had some difficulties penetrating the catheter to the right place. Naropeine and Phentanyl (2ml-100 gamma) were injected. Fifteen minutes
\end{abstract}

later, the incision took place, first at the right hip. At that moment the patient falled in an asleep state (no drugs). The acetabular component was implanted within 15 minutes from the incision. Right at that moment the anesthesiologist called for attention, since the patient was not responding to pain stimulus. Intubation was immediately performed without relaxing (myorelaxines). The operation was stopped and the team concluded that the patient was in deep coma. No laryngeal reflex, no pupilar reflex, no corneal reflex, no mydriasis, suggested us a 3 point Glasgow coma. The monitor displayed us a BP of 90/65 mmHg, HR of 100 beats in $1{ }^{`}$ (80 in 1' at start), PA Oxygen $98 \%$. A team consult with anesthesiologists, cardiologist, neurologists, and orthopedic surgeons was made immediately and possible different diagnosis were discussed (cerebral insult through an AVC or trombembolic incidence from carotis possible plaque embolisation, chemical coma- iatrogenic, etc).

Results: One hour after the onset of the coma, the intubated patient began to move the left leg with conscious movement. Five minutes later, the patient was completely waked up and was extubated. The neurologic status was perfect with no damages. We didn't loose time; we reintubated the patient under general anesthesia and the operation went on till the two hip implants implanted. The operation succeeded, the patient walked up without complications, walked the next day and was released after 7 days from the hospital.

Discussion: What happened? As a final analysis we concluded that the deep sleep was an iatrogenic complication. The punching of dura mater during the epidural anesthesia and the penetrating of Phentanyl in the CS liquor was the hypothetic cause of the temporarily deep coma. All other supposed causes were ignored because the lack of evidence in their favor.

Keyword; epidural anesthesia, myorelaxines, acetabular, dura mater... 


\section{Breast Calcification and Final Breast Cancer Diagnosis}

Gjok TOSKU ${ }^{1}$, Islam MAMICA ${ }^{2}$, Jona ISAKU ${ }^{3}$, Afrim PIRRACI ${ }^{1}$

${ }^{1}$ Radiologist at Mother Teresa University Centre; Lecturer at UMT, Albania

${ }^{2}$ Radiologist at Burrel City Hospital, Burrel, Albania

${ }^{3}$ Imaging Technician; Lecturer at FMTS, $U$ of Elbasan “A. Xhuvani”, Elbasan, Albania

\section{Abstract}

Breast calcifications are small spots of calcium salts. They can occur anywhere in the breast tissue. They are very small, so you won't be able to feel them, and they don't cause any pain.

Breast calcifications are very common and usually develop naturally as a woman ages. They are usually benign not cancer.

Having benign breast calcifications doesn't increase your risk of developing breast cancer.

Breast calcifications can occasionally be an early sign of cancer. Because of this, your doctor or nurse may want you to have further tests to check what sort of calcifications you have.

Breast calcifications are usually found by chance during a routine screening ultrasound or mammogram or during an investigation at a breast clinic for another breast problem. The calcifications show up on a mammogram as small white spots.

In many cases, a mammogram may not give the radiologists enough information. The mammogram may not be clear or they may want to check an area more carefully. However, further tests will give more information to make an accurate diagnosis. Further tests are likely to include a more detailed mammogram which gives a closeup, magnified, picture of the affected area or even a core biopsy in order to make the final diagnosis.

In conclusion, final diagnosis of breast cancer can be challenging and it may take further examinations, test and procedures.
Regular screening programs are referred as best ones for early diagnosis.

Keywords: Breast, calcifications, cancer, examinations, screening

\section{Urinary Tract Stones, Diagnostic Modalities}

Islam MAMICA ${ }^{1}$

${ }^{1}$ Radiologist, Burrel City Hospital, Burrel Albania

\section{Abstract}

The lifetime incidence of urinary tract stones is high, seen in as many as 5\% of women and $12 \%$ of males. By far, the most common stone is calcium oxalate, however, the exact distribution of stones depends on the population and associated metabolic abnormalities.

Traditionally, ultrasonography has a lower sensitivity and specificity than CT, but does not require use of radiation. However, when these imaging modalities were compared in a randomized controlled trial they were found to have equivalent diagnostic accuracy within the emergency department. Kidney, ureter, bladder (KUB) plain film radiography is most helpful in evaluating for interval stone growth in patients with known stone disease, and is less useful in the setting of acute stones. MRI provides the possibility of 3D imaging without exposure to radiation, but it is costly and currently stones are difficult to visualize.

In conclusion, developments are anticipated to enhance each imaging modality for the evaluation and treatment of kidney stones in the near future.

Keywords: Uurinary tract, stones, ultrasound, CT, KUB, MRI 


\section{Begin Tracheal Stenosis after prolonged intubation. A case reports.}

Rushan MUHAMETI ${ }^{1}$

${ }^{1}$ Pneumology service, University Hospital of

Trauma, Tirana, Albania

\begin{abstract}
Introduction: Prolonged endotracheal intubation may lead to altered tracheal blood flow, resulting in mucosal injury and scarring followed by tracheal stenosis.

Materials and Methods: We report a case of the tracheal stenosis after prolonged endotracheal intubation from balloon cuff pressures. We have provided endoscopic images and descriptions of the bronchoscopic findings along with a brief discussion of the management for this complication.
\end{abstract}

Results: (Case History) A 45-year-old man was admitted in our clinic 2 month after an accident from mine in border between Albania -Kosovo. The patient's hospital course was complicated by limited neurologic recovery, aspiration pneumonitis, and adult respiratory distress syndrome requiring an extended period of mechanical ventilation. In first presentation he has cough, moderate dyspnea and malaise. Flexible bronchoscopy was performed, first from above the tracheostomy revealing leakage of air bubbles from around an inflated cuff with underlying granulation tissue. Erosion of the mucosa overlying the tracheal rings was visualized in the region where the balloon cuff had been positioned. After 21 days the patient results with seriously tracheal stenosis and tracheal dilatation or stent was indicated. He had severe dyspnea and stridor and moderate chest pain.

Follow-up bronchoscopy revealed severe damage of the tracheal lumen associated with web-like stenosis and he has been emergency tracheal stend.

Conclusions: Specifically, patients with cuffed tracheostomy tubes are at a risk for complications of balloon over-inflation resulting in elevated cuff pressures. In the case presented, we hypothesized that the etiology of the complication described was multifactorial in nature, resulting from inadequate monitoring of the cuff pressure over an extended period of time and in the timeframe after the repair of a traumatic injury.

Keywords: cuff pressure, tracheomalacia, tracheal stenosis

\section{Psoriatic Arthritis: What Ultrasound can provide us?}

\author{
Leonard MOLLA ${ }^{1}$, Gjok TOSKU ${ }^{2,3}$ \\ ${ }^{1}$ Rheumatologist, University Hospital Center \\ "Mother Teresa", Tirana, ALBANIA \\ ${ }^{2}$ Radiologist, University Hospital Center "Mother \\ Teresa", Tirana, ALBANIA \\ ${ }^{3}$ Lecturer University of Medicine, Tirana, \\ ALBANIA
}

\section{Abstract}

Psoriases are autoimmune mediated diseases that caused raised, red, scaly patches to appear on the skin. Like psoriasis, Psoriatic Arthritis is an autoimmune disease, meaning it occurs when the body's immune system mistakenly attacks healthy tissue, in this case the joints and skin. The faulty immune response causes inflammation that triggers joint pain, stiffness and swelling.Ultrasound (US) is a valuable imaging technique for detection and characterisation of the inflammatory process in arthritides. US has widely been applied to psoriatic arthritis (PsA) in both clinical and research fields, especially focusing on enthesitis. US has proven to be useful to establish a diagnosis of PsA, to recognise subclinical involvement, (such as enthesis abnormalities in patients with PsA, and in patients with only clinically apparent skin psoriasis despite the absence of clinical symptoms of arthritis), to estimate disease activity, and to allow therapy monitoring showing structural and inflammatory changes (not only in joints and tendons, but also in domains not assessed in usual rheumatology care, such as the skin and nails). 
Key words: Psoriatic Arthritis, diagnoses, Ultrasonography, findings.

\section{Enriched Recovery for Colorectal Surgery: Discussions and Forthcoming Challenges}

\author{
Henri KOLANI ${ }^{1}$ \\ ${ }^{1}$ Surgery Service, University Hospital Center \\ "Mother Theresa", Tirana, ALBANIA
}

This could be obtained in example by evidencing those social factors that can delay the discharge and therefore organizing the available resources outside the hospitals well in advance the operation. Keywords: Colorectal, surgery, recovery, protocols

\section{Trend of Implantology}

\author{
Arben MUCAJ ${ }^{1}$, Bashkim MURATI ${ }^{1}$ \\ ${ }^{1}$ OroMaxilloFacial Surgeon, and University \\ Lecturer, Tirana Albania.
}

\section{Abstract}

The advancement in implant dentistry has led to a significant improvement in the quality of life of our patients; however, there are still many controversies and many unanswered questions.

The trend is towards minimally invasive surgical procedures with cost effective dental rehabilitations although many of these therapeutic approaches are still under scrutiny. For instance, till date, the scientific community does not have a definitive answer to the following issues: short implants or bone reconstructions and longer implants, the best reconstructive approach, the ideal implant surface, systemic factors negatively influencing implant cluster failures, the most appropriate treatment for perimplantitis and the ideal aesthetic materials for implant prosthesis, to list a few. Additionally, clinical and ethical issues including when teeth should be extracted and implants placed, are also unclear. Over the years, we have seen in meetings and congresses, a dramatic increase in the extraction of teeth that could be saved. A defined algorithm for such instances has not yet been presented and the controversy still remains open.

Translating evidence into treatment predictability should be the goal in implant dentistry in the near future, with emphasis on an open and honest transparent system that provides the complete financial disclosure of every author. This is essential care and not to shorter recovery periods. 
in a field like implant dentistry where conflicts of interest could generate confounding factors and bias.

Keywords: Implant, dentistry, minimally invasive, surgical procedure

\section{Assessment of Single Implants in Mandibular Area Under Immediate Loading}

Bashkim MURATI ${ }^{l}$, Arben MUCAJ ${ }^{1}$

${ }^{\text {I}}$ OroMaxillo Facial Surgeon, and University Lecturer, Tirana, ALBANIA.

\begin{abstract}
The aim of this study was to evaluate the survival of single dental implants subjected to immediate function. Twelve patients with edentulous areas in the posterior mandible were included in the study. All received at least one regular platform dental implant $(3.75 \mathrm{~mm} \times 11 \mathrm{~mm})$. Clinical and radiographic parameters were evaluated. The survival rate after 12 months was $83.3 \%$. The implants showed no clinical mobility, had implant stability quotient values (ISQ; Osstell) around 50, bone loss of up to $2 \mathrm{~mm}$, and a probing depth of $\leq 3 \mathrm{~mm}$. Although the posterior mandible is an area in which the immediate loading of dental implants should be performed with caution, this treatment presented a good success rate in the present study sample.
\end{abstract}

Keywords: Dental implants, posterior mandible, evaluate, treatment

\section{Airways Management in a Child with Foreign Body Airway Obstruction. A Case report.}

Brikena DAUTAJ $^{l^{*}}$, Enriketa $\mathrm{JAHO}^{l}$, Rexhina STURCE $^{l}$, Bardhyl KURTI ${ }^{l}$, Esmerilda BULKU ${ }^{l}$

${ }^{1}$ Anesthesia and intensive Care Service, University Hospital of Trauma, Tirana, Albania.

\section{Abstract \\ Children may require intubation for unique reasons, and pediatric intubation in emergency room can create challenges to}

the anesthesiologist. There are differences between the adult and pediatric cardiopulmonary systems, the children have a higher risk for respiratory failure, so the emergency is immediately.

In our Emergency room is admitted a little boy about two years old (brought from his parents) with an acute respiratory failure. The boy was with a terminal breathing, with takipnea and superficial breathing, without conscience, with cyanosis ( $\mathrm{SpO} 2$ $50 \%$ ). The parent told us that the child is choked with cherries. Immediately with the help ofa videolaringoscopy we see a big cherry in the entrance of the epiglotic that made difficult the procces of breathing to the child. To not lose time we decided that was better to assure first the airways in putting a tracheal tube and ventilating him, so we had time to put off the cherry from the orofaringeal site, then we put off the cherry with the help of a cleme meanwhile the child was being ventilates with $100 \%$ of $\mathrm{O} 2$. After this we assured an intravenous route and sedated the child with propofol. In the CT scanner made to the child we sow a pneumonia, so we decided to let him in mechanical ventilation, with PEEP $3 \mathrm{~cm}$ $\mathrm{H} 2 \mathrm{O}$, corticotherapy and antibiotherapy. After 12 hours the child was extubated, he was quite, conscient without signs of respiratory failure, He got out from the hospital after two days.

\section{Femoral Neck Fracture}

\author{
Zamir DEMIRAJ ${ }^{1}$, Anxhela AHMATAJ ${ }^{2}$ \\ ${ }^{1}$ Orthopedic service, University Hospital of \\ Trauma, Tirana, Albania \\ ${ }^{2}$ Physical Therapist, University Hospital of \\ Trauma, Tirana, Albania
}

\begin{abstract}
Background: Neck of femur fractures (NOF) are common injuries sustained by older patients who are both more likely to have unsteadiness of gait and osteoporosis (reduced bone mineral density). Femoral neck fracture in this age-group pose a
\end{abstract}


major problem in three aspects: medical, economy and social.

It is recommended to intervene in the early 48 first hours, naturally if there is no contraindication, as the result of comorbidities which can risk even the patients' life.

Surgical treatment of neck of femur fractures is easier in this age-group and it includes: partial or total prosthesis. The most important thing is the return of patient in the ADL (activity daily life) after surgery.

The femoral neck fractures are common even in the adult and pediatric age due to high energy trauma, like falls from height or car crashes. The main problem for medical team consists in the chosing of surgical treatment option. Between osteosinthesys and prosthesis we would naturally choose the osteosinthesys in pediatrics and young adults.

Another problem based on the classification of these fractures is whether we always are successfully in our choice, to avoid two of the complications of osteosinthesys: pseudoarthrosis and avascular necrosis of femur.

The difficulty of choosing is higher between the ages 55-65. A lot of studies heve been done for this problem. The importance stands in the fact that the disruption of blood supply to the femoral head can be evidented, which is dependent on the type of fracture and causes significant morbidity, diagnosis and classification of these fractures. These studies are in experimental phases yet. The article aims to give a review of literature concerning femoral neck fractures and their surgical treatment options

Key words: fracture, femoral neck, osteosynthesis, prosthesis.

\section{Congenital Diafragmatic \\ Hernia: Segment Hepatic \\ Herniation Through a Diafragm \\ Defect Simulating Basal Lung \\ Nodes}

Fadil GRADICA ${ }^{1}$, Lutfi LISHA ${ }^{1}$, Dhimitraq ARGJIRI $^{3}$, Agron DOGJANI ${ }^{2}$, Alma CANI ${ }^{4}$, Fahri KOKIÇ $^{4}$, Ylber VATA ${ }^{1}$, Leon SHPATARAKU ${ }^{1}$, Dori BOZAXHIU ${ }^{1}$.

${ }^{I}$ Thoracic and visceral Surgery Service, University Hospital "Shefqet Ndroqi" Tirana, Albania.

${ }^{2}$ General Surgery and Trauma Service, University Hospital of Trauma, Tirana, Albania.

${ }^{3}$ Pneumology Service, University Hospital "Shefqet Ndroqi" Tirana, Albania.

${ }^{4}$ Anesthesia \& Intensive Care Service, University Hospital "Shefqet Ndroqi" Tirana, Albania.

\section{Abstract \\ Background: Congenital diaphragmatic} hernia is a major malformation occasionally found in newborns and babies. Congenital diaphragmatic hernia is defined by the presence of an orifice in the diaphragm, more often to the left and posterolateral that permits the herniation of abdominal contents into the thorax.

The aim of this case series is to provide information on the presentation, diagnosis and outcome of two patients with latepresenting congenital diaphragmatic hernias. The diagnosis of congenital diaphragmatic hernia is based on clinical investigation and is confirmed by plain Xray films and computed tomography scans. Materials and Methods: We present three case reports of asymptomatic abdominal viscera herniation within the thorax. The first case concerns a rare type of herniation in the right side of the thorax of the right hemithorax a part of the liver parenchyma in a 65-year-old woman and left side liver abscess. The second case concerns herniation of the part of liver into the left hemi-thorax in a 37-years old woman. The third case concerns herniation of the stomach and bowel,omentum and spleen into the left side of the chest with compression of the left lung in a 55-yearold man. This type of hernia may appear later in life, because of concomitant respiratory or gastrointestinal disease, or it 
may be an incidental finding in asymptomatic adults.

Conclusions: Patients who present with late diaphragmatic hernias complain of a wide variety of symptoms, and diagnosis may be difficult. Additional investigation and research appear necessary to better explain the development and progression of this type of disease. Surgery is treatment of choice.

Keywords: Congenital diaphragmatic hernia, surgical treatment, hernioplastics

\section{Management of Emergency Splenectomies. Our Experience in the Regional Hospital of Durres.}

Agron DOMI ${ }^{1 *}$, Denis GODAJ ${ }^{1}$, Shkelqim FERKO $^{1}$, Fitim SHINI ${ }^{1}$, Anton NDOJ ${ }^{1}$

${ }^{l}$ Surgery Department, Regional Hospital of Durres, Albania

\section{Abstract \\ Introduction: The aim of this two years retrospective, monocentric study (held in Department of Surgery, Regional Hospital of Durres) is to describe the demographic, circumstances and etiology, clinical and diagnostic features, grade of injury as well as treatment of patients who underwent emergency splenectomy. The spleen is the most often injured solid organ in trauma.}

Methods and Methods: We conducted the study in the interval between 01.09.2014 and 01.09.2016. We analyzed 18 clinical charts of hospitalized patients who underwent emergency splenectomy in our hospital.

Results: Demography: 18 patients as 13 Male (72\%) and 5 females (28\%).Study group's average age of patients is $29( \pm 12)$ years old, males $26( \pm 14)$ and females 39 $( \pm 7)$. Etiology: car accident (politrauma) 10 patients, closed trauma (falling, physical attack etc.) 5 patients, blunt trauma 3 patients. Diagnose: All patients underwent abdominal CT and US, TBC. Injury grade was based in the CT and US examination. Grade I- 4 patients, Grade II
- 4 patients, Grade III -3 patients, Grade IV -6 patients, Grade V - 1 patient.

Treatment: 15 patients underwent splenectomy, 3 patients underwent splenorrhaphy.

Conclusion: Spleen injury is a common emergency in our department. Usually it is not an isolated problem and is included in the treatment of a politrauma. Mostly affects young patients in the second and third decade of life. Considering the important role of the spleen in the immunologic system a more careful evaluation should be made whether to perform splenectomy in low grade injuries. Keywords: emergency splenectomies, management, trauma

\section{Our Experience Using C- Mac Videolaringoscope Device for Intubation}

Silvana LEKA ${ }^{1 *}$, Rexhina STURCE ${ }^{1}$, Flutura BALLA $^{2}$,

${ }^{1}$ Department of Anaesthesiology-Intensive Care, University Hospital of Trauma, Tirana, Albania.

${ }^{2}$ Anaesthetist Nurse, University Hospital of

Trauma, Tirana, Albania.

\begin{abstract}
The management of airway is the first thing to do when you a deal with a trauma patient. Often, the situation is not easy, not only because the politraumatized patient can be a high score Mallampati, but the mouth full of blood, mandibular- maxillae fracture, the need for not moving the head in extension cause of the neck immobilization are our everyday theatres. When intubation of this patients is needed, direct laryngoscopy is not always possible. From almost one year and a half our hospital provided the $\mathrm{C}$ Mac videolaryngoscope and all the anaesthesiologists staff feel more secure in everyday work.

The device is a portable, battery operated video laryngoscope that resembles a standard Macintosh blade. The intubation technique is identical to conventional Macintosh laryngoscopy with the
\end{abstract}


additional aid of a magnified video view for the difficult intubations.

From our experience and from a lot of randomized control trials in the difficulty intubation is demonstrated that C-MAC device offers a better laryngeal view, more successful intubations on the first attempt, shorter laryngoscopy and intubation times, decreased needs of adjuncts, exert less force on maxillary incisors The C-MAC videolaryngoscope is a very good tool for tracheal intubation teaching and as a university hospital we really love it.

As for every new technology, with videolaryngoscopy, the main challenges for the intubator are to become familiar with the view on the monitor and coordinate the eyes and hands appropriately. As a conclusion in our hospital we have included this device in the initial step of difficult airway management. 
A Arapi, 68

A Dizdari, 97

A. Bejleri, 11

A. Koni, 35

A. Lenjani, 19

A. Nake, 109

A. Nurçe, 109

A. Pere, 82

A. Simaku, 130

A. Veseli, 130

A.Sinaj, 72

Adamantia Koutava, 36

Admir Mustafa, 64, 69

Adrian Hoti, 64

Adrian Nelaj, 15, 100

Aferdita Ademi, 71

Afrim Pirraci, 139

Afrim Tahiri, 115

Afron Mici, 82

Agron Dogjani, 10, 44, 64, 82, 86, 101, 102,

$111,125,131,133,134,143$

Agron Domi, 144

Agron Menzelxhiu, 82

Aida Lako, 91

Ajruli B, 132

Alan Andonovski, 93, 96

Alban Hysenaj, 97

Alban Rushiti, 111

Albana Aleksi, 28, 121

Albana Doko, 103

Albana Kenga, 127, 128, 129

Albana Kreca, 103

Albana Shahini, 65

Albenc Dervishi, 90

Albert Pesha, 35

Albulena Baloku, 126

Alda Kika, 46

Aleksandar Saveski, 50, 52, 132

Aleksandar Trajanovski, 50, 52, 132

Aleksander Hoxha, 27

Alesio Bocari, 16

Alfred Gega, 51

Alfred Ibrahimi, 27

Ali Devaja, 15, 126

Alma Cani, 28, 82, 143

Alma Llukacaj, 117

Almir Skana, 67

Altin Habazaj, 137

Altina Xhaferi, 66

Aman Gapbarov', 116

Amarildo Blloshmi, 12,131

Anastasika Poposka, 97

Andonovska B, 93,96

Andrijan Kartalov, 63
Anila Belchishta, 97, 135

Anila Racaj, 61

Anisa Liço, 123

Anton Milo, 86

Anton Ndoj, 35, 144

Antonio Catona, 13

Antonio Gavrilovski, 50, 52, 132

Anxhela Ahmataj, 71, 142

Apostol Vaso, 48

Arben Beqiri, 13

Arben Gjata, 9, 123, 124

Arben Gjonej, 72, 73

Arben Haxhihyseni, 83

Arben Mucaj, 141, 142

Arben Rroji, 46

Arber Demiri, 31

Arbëresha Gurguri, 73

Ardiana Sinani, 108, 109

Ardita Osmani, 71

Arian Hamiti, 109

Arian Hodo, 34

Arjan Mezini, 106

Arjana Sina, 68

Arlind Myftari, 102

Arnel Mici, 49, 77, 97

Arsen Seferi, 46

Arsim Ajeti, 15, 126

Arta Kushi, 102, 103

Artan Gjika, 64, 69

Artid Duni, 51, 52, 97

Artid Lame, 46, 47

Arvin Dibra, 15, 114, 125

Arvit Llazani, 14

Asead Abdyli, 29, 37

Astrit Hoxhaj, 69

Astrit Kalenja, 82

Astrit Mustafa, 34, 36, 101

Astrit Peka, 72, 73

Astrit Xhemali, 15

Athanasios Lazaridis, 34

Aulona Haxhirexha, 102, 106

Avenir Balili, 84

Ayman El-Menyar, 22, 62, 80

Bajram Begaj, 105

Bardhyl Kurti, 142

Bashkim Murati, 141, 142

Basri Lenjani, 19, 31, 56, 57

Baton Kelmendi, 111, 112

Bedri Braha, 115

Bedri Mihaj, 34

Berat Lenjani, 19, 56, 58

Besim Boci, 16

Besmir Bulku, 68

Bilbil Hoxha, 35 
Blerim Arapi, 29, 37

Blerim Fejzuli, 71

Blerim Zeqiri, 22

Blerina Bregu, 18

Blerta Hamolli, 49, 77

Blerta Hasalla, 87

Boris E. Sakakushev, 24, 99, 114, 115

Braha B., 15

Brikena Dautaj, 142

Carlos Mesquita, 43

Cem Ilgin Erol, 116

Claudio Taglia, 16

Constantinos Adamou, 36

D Ferizi, 35

D. Selmani, 109

D. Xhemalaj, 82

Dafina Matoshi, 111

Damla Beyazadam, 116

E. Daniela Bimi, 70, 74

F. Daniela Georgieva, 97

G. Daniela Vercuni, 83

Danny Daphnis, 84

Dardan Lenjani, 31, 56, 58

Dariel Thereska, 14, 123

Deniona Nunci, 14

Denis Godaj, 144

Denis Kosovrasti, 127, 128, 129

Devid Belalla, 123

Dhimitraq Argjiri, 82, 106, 143

Dimitrios Kotsiris, 36

Dominika Vrbnjak, 25, 120

Dorela Vasha, 108, 109

Dori Bozaxhiu, 143

Dorian Habili, 60, 97

Dorian Meta, 75

Dorina Shtjefni, 45, 55

Drini Shehi, 15, 125

Dritan Cobani 10

Dritan Todhe, 59, 93, 138

Doruntina Klenja 133

E. Shima, 28

Earta Gega, 101

Eda Luzaj, 32

Edison Halili, 128

Edison Ndreka, 32

Edlira Kukeli, 58

Edmond Kapedani, 127, 130

Edmond Nuellari, 127, 128, 129, 130

Edmond Pistulli, 109

Edvin Prifti, 79, 80

Edvin Selmani, 51, 73, 94, 95

Ejona Celiku, 101

Ejona Lilamani, 46

Elfije Krasniqi, 56, 57

Eljona Xhelili, 15

Elona Gjylbegu, 85
Elona Hasalla, 87, 89

Elona Markeci, 55, 101

Elona Naqellari, 40

Elvi Sota, 91

Elvina Shaba, 105

Emilija Stojkovska-Pemovska, 76

Emiljano Pjetri, 12

Emir Q. Haxhija, 44, 81

Engjellushe Jonuzi, 44

Eni Mehmeti, 68

Enkeleda Sinaj, 91

Enkelei Balla, 79

Enriketa Jaho, 142

Enton Bollano, 14, 123

Enver Fekaj, 11, 115

Erald Xhelili, 30

Ergys Cami, 51, 52, 97

Erion Spaho, 47

Erisa Mane, 87

Eriselda Taulla, 105

Erjona Zogaj, 75

Ermira Muco, 102, 103

Ermira Pajaj, 46, 47

Erton Karafili, 66, 67

Ertugilda Hoxhaj, 32

Ervin Bejko, 129

Erza Voca Mulaj, 19,31, 56

Esen Uysal, 31, 56, 57

Esmerilda Bulku, 30, 142

Etmont Celiku, 10, 15, 100, 101, 117, 124

Eugen Tata, 37

Evangelos Liatsikos, 36

Evisa Zhapa, 36

F. Krasniqi, 109

Fadil Gradica, 28, 82, 106, 143

Fahri Kokiçi, 82, 143

Fahri Kokiçi, 28

Fatime Lenjani, 19

Fatjona Kamberi, 73, 88

Fatjona Shehu, 133

Fatmir Brahimi, 94, 95

Faton Hoxha, 15

Faton T. Hoxha, 126

Fausto Catena, 41, 54, 62

Ferat Sallahu, 11

Ferit Muharemi, 124

Ferizat Dika - Haxhirexha, 102, 106, 125

Fitim Shini, 144

Floren Kavaja, 15

Flutura Balla, 144

Fotios Dimitriadis, 34

Francesco Saverio Grossi, 36

Gani Çeku, 111, 112

Gani Shabani, 31, 56, 57

Gazmend Zylbeari, 102

Genc Kabili, 84, 86 
Gentian Huti, 29, 37

Gentian Zikaj, 120

Gentjana Haskja, 88

Gezim Galiqi, 35

Gezim Guri, 64, 69

Gezim Xhepa, 120

Gjergji Belba, 120

Gjergji Caushi, 51, 52, 97

Gjergji Çaushi, 49

Gjergji Semini, 34, 36

Gjok Tosku, 139, 140

Gjorgji Jota, 63

Gledion Fejzo, 47

Goran Begovic, 63

Gramoz Brace, 46, 47

Granit Ramizi, 134

Gresjeta Vata, 32

Grigorios Daligaros, 34

Guido Bassi, 51, 52

H. Gjergo, 130

Hava Vangjeli, 32

Hektor Sula, 26

Helidon Nina, 82

Henri Kolani, 15, 100, 101, 141

Herion Dredha, 37

Hysni Bendo, 12, 111, 133

Hysni Jashari, 111, 112

Ibrahim Avdiu, 82

Igor Kaftandziev, 95

Ilaz Miftari, 124

Ilber Besimi 124

Ilda Taka 87

Ilia Mazniku, 58, 76, 87

Ilir Alimehmeti, 104

Ilir Hasani, 8, 9, 21, 40, 42, 50, 52, 61, 92, 93, $95,96,132$

Ilir Hasmuсa, 78, 97

Ilir Kajo, 105

Ilir Lumi, 66, 75

Ilir Shabani, 93

Ilir Shani, 64

Ilir Ukimeri, 126

Imri Vishi, 25, 36

Indrit Bimi, 70, 74

Indrit Jaupaj, 30, 33

Inida Ruli, 123

Irena Kola, 91

Irena Shala, 12

Irena Tresa, 90

Irnis Biščo, 110

Islam Mamica, 139

J. Profili, 68

Jahja D, 52

Jakup Vrioni, 17

Jasenka Škrlin, 98

Jasmina Kröpfl, 25, 120
Jasmina Veseli Arslani, 122

Jetlir Rashiti, 126

Jetmira Kerxhalliu, 46, 47

Jeton Shatri, 20

John E. Francis, 37, 118

Jona Isaku, 72

Jonida Mehmeti, 102, 103

Jonida Tula, 106

Jordan Saveski, 8, 21, 40, 76, 92

Jovica Ugrinovski, 20

Julian Kraja, 89

Kamnar V, 52

Kastriot Haxhirexha, 44, 102, 111, 125

Kenan Karavdić, 110, 118

Kilda Gusha, 12

Kimete Kadesha, 90

Kiril Lozanche, 20

Kledisa Harizi, 56, 58

Klejdi Latifi, 91

Klevis Thomai, 16, 17

Klodian Hyka ${ }^{1}, 86$

Korab Sejdini, 51, 52, 97

Kostandinos Zotos, 34

Krenar Lilaj, 14

Krenar Preza, 67

Kreshnike Dedushi, 69

Kujtim Alimeri, 64, 69

L. Agolli, 28

Lazo Jovcheski, 59

Leard Duraj, 94, 97

Leart Berdica, 107, 108

Ledia Kaci, 106

Ledian Fezollari, 51, 52, 97

Leon Kaza, 85, 86

Leon Shpataraku, 143

Leonard Molla, 140

Lindita Saja, 91

Liridon Nuredini, 135

Ljupco Nikolov, 95

Ljuzim Agai, 20

Lorena Haxhihyseni, 17, 18

Loreta Agolli, 106

Luan Nikollari, 11

Luljeta Stanaj, 49, 61

Lulzim Q. Maloku, 126

Lutfi Lisha, 82, 143

Lutfi Zhegu, 82

Lutfi Zylbeari, 102, 125

Majlinda Naço, 117

Maksim Çela, 66, 67

Maksim Gjoni, 85

Manushaqe Saraçi, 49, 61

Marija Atanasova, 63

Marinela Kumaraku, 113

Marion Sako, 18

Marjan Kamiloski, 61 
Marjeta Tanka, 112

Marjola Qatipi, 77

Marko Spasov, 95

Marsela Sopiqoti, 129

Mauro Zago, 10, 23, 43

Medien Xhaferi, 66

Mentor Petrela, 22, 46

Merita Zeka, 27

Miftari F, 15

Migena Ishmakej, 131

Mihal Kërçi, 40

Milan Samardziski, 52

Mirel Grada, 46

Mishel Qirinxhi, 47

Mustafa Xhani, 37

Myfit Saraci, 46

Myftar Torba, 55

Myriam Hunink, 104

Myzafer Kaçi, 15, 125, 129

N. Elshani, 64

Naim Dizdari, 101

Naime Çullhaj, 90

Nardi Kola, 120, 121

Natasha Merko, 64, 91

Neada Hoxha, 103

Neada Hoxha, 102

Neda Trajkovska, 8, 21, 40, 76, 92

Nehat Baftiu, 38, 39

Neritan Myderrizi, 53

Nexhmi Hyseni, 111, 112

Niketa Kolici, 85

Nikollaq Kaçani, 123, 124

Norik Bardhi, 46, 47

Nuhi Arslani, 13, 99, 122

Oliver Arsovski, 95

Orhan Alimoglu, 24, 55, 62, 116

Orjada Gashi, 17, 18

Ornela Çela, 134

Ornela Nuredini, 106

Ozgur Ekıncl, 25, 116

Panagiotis Kallidonis, 36

Panteleimon Ntasiotis, 36

Pascal Gervaz, 63

Petraq Mustaqe, 121

Petrika Gjergo, 127, 128, 129, 130

Petrit Biberaj, 103

Philippe Konstandinidis, 63

Pirro Prifti, 38

Premtim Rashiti, 56, 57

Preveza Abrashi, 115

Q.Arapi, 72

Qemal Rushiti, 63

R. Baftiu, 39

R. Baftiu, 38

Ramadan Sopa, 126

Reshat Mati, 126
Rexhep Selmani, 63

Rexhina Sturce, 142, 144

Rezar Deveja, 27

Rezart Xhani, 37

Rezeart Dalipi, 50, 52, 96, 132

Ridvan Alimehmeti, 46, 47

Rifat Bajrami, 115

Rifat Latifi, 7, 23, 53, 98, 111

Rinard Kortoci, 29, 37

Risida Gjonej, 72, 73

Risto Cholanchevski, 135

Rovena Bode, 100

Rovena Lico, 108

Rrustem Musa, 15, 126

Rudin Domi, 26, 29

Rudina Degjoni, 12

Rufadije Vejseli, 124

Rushan Muhameti, 140

Rustem Celami, 84, 85, 86

Sadi Bexheti, 20, 102

Saimir Heta, 113

Salih Grajcevci, 112

Samardziski M, 132

Sami Bytyci, 63

Sasa Milenkovic, 42

Selda Veizaj, 91

Selim Çerkezi, 20

Selim Horeshka, 40

Serbeze Kabashi Mucaj, 69

Serdi Memini, 51, 97

Sevdije Koxha, 106

Shahini L, 15

Shemsedin Hashani, 15, 126

Shkelqim Ferko, 35, 53, 144

Shkelqim Met'hasani, 27

Shkelzen Osmanaj, 44, 82, 101

Shpetim Ymeri, 35

Shpresa Hashimja, 126

Shqipe Luta, 103

Silvana Leka, 144

Simon Trpeski, 22, 50, 59, 132

Skender Brataj, 30, 32, 33

Skënder Brataj, 56, 57

Skender Buci, 45, 55, 82

Skender Saidi, 33

Skender Veliu, 25, 120

Skender Zatriqi, 11, 115

Sokol Buba, 112

Sokol Isaraj, 119, 120, 121

Sokol Ruci, 55

Sokol Shehu, 27

Sokol Xhepa, 127, 128, 129

Sonja (Saraçi) Butorac, 64, 69

Stavri Llazo, 129

Stephan Frenzel ${ }^{2}, 7$

Stojan Potrči, 13 
Sulejman Baha, 87

Sulejman Sokoli, 122

Tatjana Spirovska, 63

Teodora Todorova, 50, 52, 132

Teona Bushati, 107, 108

Teuta Abazi, 124

Thoma Qirjazi, 27

Thomas B. Whittle, 54, 126

Tunc Eren, 116

Valbona Selmani, 94

Valdete Kaloshi, 58

Vangjush Lekgjika ${ }^{l}, 86$

Vasil Miha, 106

Vera Gjinaj, 12

Vesel Skenderi, 115

Veton Ademi, 20

Vidi Demko, 64, 68, 69

Vilma Cadri, 103

Vilmos Vécsei, 7, 41
Vilson Ruci, 51, 52, 97

Viola Selmani, 106

Viola Shuteriqi, 112

Violeta Zatriqi, 11

Vrenoz Hodaj, 137

Xheladin Draçini, 100, 117

Xhesika Xhetani, 64

Ylber Vata, 82, 143

Ylber Zama, 97

Ylli Ziqishti, 82

Ymer Durmishi, 63

Zamir Demiraj, 51, 71, 77, 142

Zamira Bexheti, 102

Zamira Shabani, 12

Zef Delia, 86

Zojë Demaj, 92

Zoran Bozhinovski, 50

Zoran Karadzov, 63 



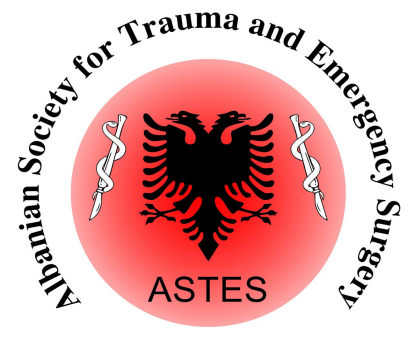

\title{
Organised by
}

Albanian Society for Trauma \&

Emergency Surgery (ASTES)

$3^{\text {rd }}$ Annual Albanian Congress of Trauma \& Emergency Surgery - ACTES 2019

\author{
November 7 - 9, 2019
}

Tirana International Hotel

Tirana, Albania

\section{SAVE THE DATE}

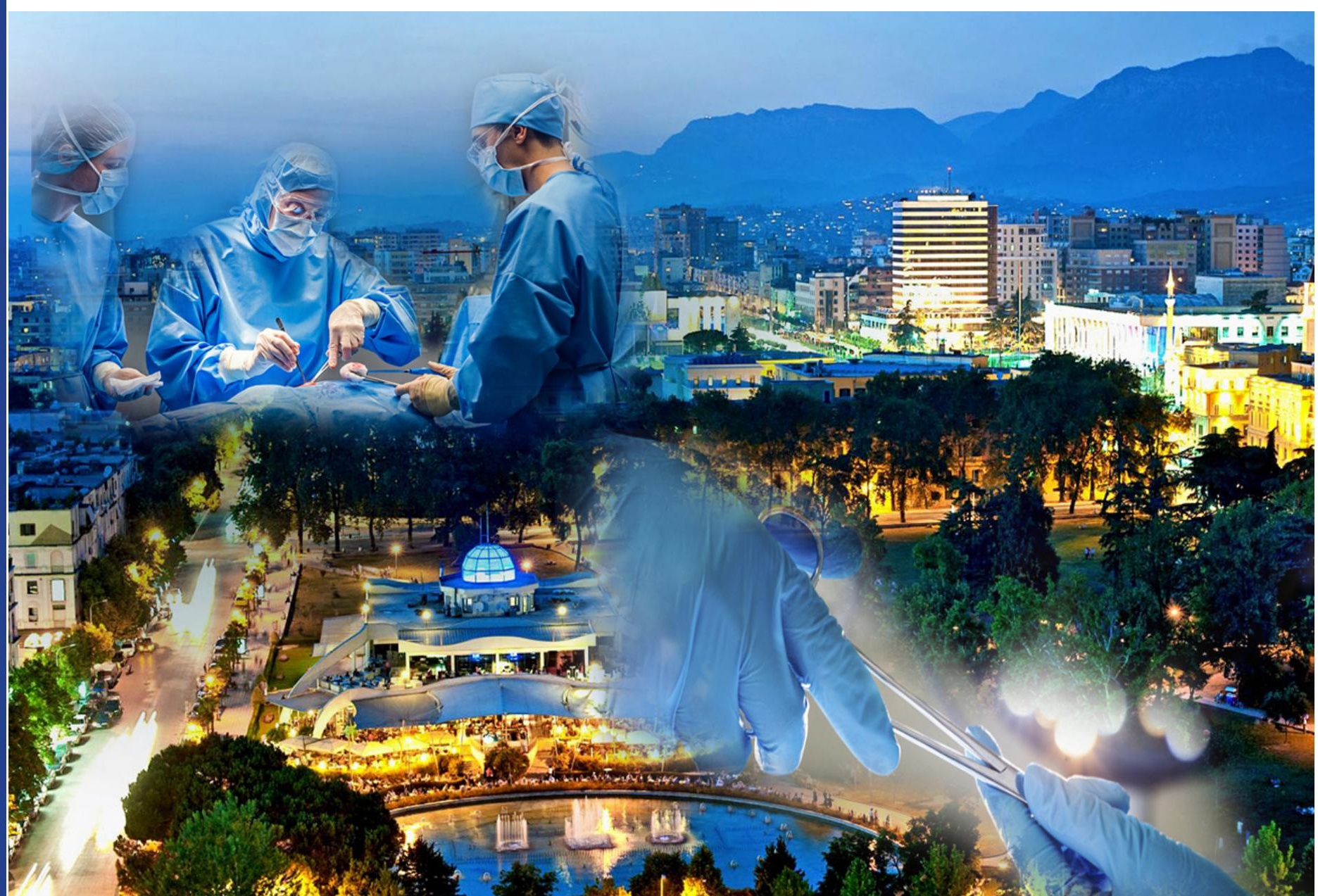

